\title{
Cation induced self-assembly of intermediate filaments
}

\author{
Dissertation \\ for the award of the degree \\ "Doctor rerum naturalium" \\ of the Georg-August-Universität Göttingen \\ submitted by \\ Martha Elisabeth Brennich \\ from München (Germany)
}

Göttingen (2012)

Institute for X-Ray Physics

Georg-August-Universität Göttingen 


\section{Thesis Committee:}

Prof. Dr. Sarah Köster (supervisor and 1st reviewer)

Georg-August-Universität Göttingen

Physics Department

Institute for X-Ray Physics

Prof. Dr. Eberhard Bodenschatz (2nd reviewer)

Max-Planck Institute for Dynamics and Self Organization

Department Fluid Dynamics, Pattern Formation and Biocomplexity

Prof. Dr. Tim Salditt

Georg-August-Universität Göttingen

Physics Department

Institute for X-Ray Physics

\section{Date of oral examination:}

11.07.2012 


\section{Abstract}

Cellular shape and the internal structure of living cells are defined by a dense network of three independent filament systems (actins, microtubules and intermediate filaments) termed "the cytoskeleton". Of these three systems, intermediate filaments (IFs) are the most genetically diverse class. A unifying feature of IFs is their formation from extended coiled-coil shaped proteins. Vimentin is one member of the IF protein family that is found in cells of mesenchymal origin such as fibroblasts. In vitro, vimentin filaments assemble hierarchically from tetrameric precursors of about $5 \mathrm{~nm}$ diameter and $60 \mathrm{~nm}$ length, which form stable complexes at low ionic strength, to several mircometers long, $10 \mathrm{~nm}$ wide filaments. This assembly process can be initiated by increasing the ionic strength, e.g. by the addition of monovalent cations, i.e. sodium or potassium. The hierarchical assembly process includes several stages, such as the formation of unit length filaments (ULFs, about $17 \mathrm{~nm}$ diameter and $60 \mathrm{~nm}$ length). Here, we investigate how the cross-section of both filaments and intermediate stages of the assembly process changes for different cation concentrations.

Small angle X-ray scattering (SAXS) allows us to study the structures of macromolecules in solution. In order to access intermediate stages of the assembly process in situ, we design and characterize microfluidic mixers which are compatible with SAXS. These mixers allow for precise control of the ionic environment of intermediate states.

For vimentin filaments, we observe an increase of diameter with increasing monovalent ion concentration. Divalent ions, such as magnesium, result in significantly thicker filaments and a qualitatively different cross-sectional structure. In addtion, we are able to identify the ULF stage of assembly in situ and to show that its diameter also increases with increasing potassium concentration.

The results of this thesis help to deepen our understanding of the structural changes of vimentin during the later stages of the filament assembly process, thereby providing a building block in the picture of how changes in the vimentin network affect the mechanical properties of cells. 



\section{Contents}

1 Introduction $\quad 1$

2 Intermediate filament formation - a self-assembly process 3

2.1 Vimentin, an intermediate filament protein . . . . . . . . . . 3

2.1.1 Vimentin ..................... 4

2.1.2 Kinetics of vimentin assembly in vitro . . . . . . . . 7

2.2 Self-assembly processes . . . . . . . . . . . . . . . . . . 8

2.3 Reconstitution of vimentin . . . . . . . . . . . . . . . . . 9

3 Small angle X-ray scattering - a probe for macromolecular struc$\begin{array}{ll}\text { ture } & 11\end{array}$

3.1 Advantages and applications of SAXS . . . . . . . . . . . . . . 11

3.2 General considerations . . . . . . . . . . . . . . . . . . 13

3.3 Acquisition of SAXS data . . . . . . . . . . . . . . . 15

3.3.1 Steady state experiments at DORIS III - B1 . . . . . . . . 15

3.3.2 Microfluidic experiments at SLS - cSAXS . . . . . . . . 17

3.4 Interpretation of SAXS curves . . . . . . . . . . . . . . . 19

3.4.1 Model-free analysis . . . . . . . . . . . . . . . . . . . . 19

3.4.2 Model-based analysis . . . . . . . . . . . . . . . 21

4 The effect of cations on the equilibrium state of vimentin 23

4.1 Reproducibility of results . . . . . . . . . . . . . . . . . . 23

4.2 Influence of protein concentration . . . . . . . . . . . 25

4.3 Influence of salt concentration . . . . . . . . . . . . . . 28 
4.4 Quantitative analysis . . . . . . . . . . . . . 36

4.4.1 Model-free analysis . . . . . . . . . . . . . . . . . 38

4.4.2 Form factors of structural models . . . . . . . . . . . . . 47

4.5 Interpretation . . . . . . . . . . . . . . . 63

4.5.1 Effect of protein concentration . . . . . . . . . . 63

4.5.2 Effect of changes in the ionic compositions . . . . . . . . . 65

5 Microfluidics for SAXS $\quad 75$

5.1 Flow at low Reynolds numbers . . . . . . . . . . . . . . . . . 75

5.1 .1 Viscous flow . . . . . . . . . . . . . 76

5.1 .2 Distribution of solutes in flow . . . . . . . . . . . 77

5.2 Microfluidic mixers . . . . . . . . . . . . . . . . . . 78

5.3 Avoiding surface effects . . . . . . . . . . . . . . . . . . . 80

5.4 Accessing reactions in flow by SAXS . . . . . . . . . . . . 81

5.5 Fabrication of microfluidic devices . . . . . . . . . . . . . . 83

5.5.1 Photolithography ................. 83

5.5.2 Casting of devices from the master wafer . . . . . . . . . 85

5.5.3 Connection to the syringe system . . . . . . . . . . . 87

5.6 Characterization of solute distribution with fluorescein . . . . . 88

5.7 Assembling vimentin in microflow . . . . . . . . . . . . . . . . . 90

6 Modeling of SAXS curves from vimentin assembly in flow $\quad 93$

6.1 Simulation of flow field and concentration distributions . . . . . . . . 93

6.1.1 Simulation system _. . . . . . . . . . . . . . . . 94

6.1.2 From simulations to finite probe volumes . . . . . . . . . 96

6.1.3 Exemplary simulation results . . . . . . . . . . . 97

6.1.4 Extrapolating beyond the simulation volume . . . . . . . . 116

6.2 Predicting SAXS curves from the simulations . . . . . . . . . . 117

6.2.1 Instantaneous filament formation . . . . . . . . . . . . 117

6.2.2 Instantaneous ULF formation and elongation . . . . . . . . . 120 
6.2.3 Instantaneous ULF formation without elongation . . . . . . . 121

6.2.4 From tetramer to filament including kinetics . . . . . . . . . 123

7 Assembly of vimentin in microflow $\quad 127$

7.1 Exemplary results . . . . . . . . . . . . . . . . . . . 127

7.2 Quantitative analysis . . . . . . . . . . . . . . 140

7.3 Interpretation . . . . . . . . . . . . . . . . . 147

8 Discussion of biophysical context 155

8.1 Meta-stability of the ULF state . . . . . . . . . . . . . . . . 155

8.2 Comparison to other biopolymer systems . . . . . . . . . . . 158

9 Conclusions $\quad 161$

10 Outlook 163

A Purification of vimentin 165

A.1 Culture of E.coli . . . . . . . . . . . . . . . . . . . 165

A.2 Extraction of vimentin from the E.coli pellet . . . . . . . . . . 166

B Derivation of form factors for SAXS 171

B.1 Seperation of longitudinal and cross-sectional components . . . . . . 171

B.2 The effect of longitudinal polydispersity on the form factor . . . . . . 172

B.3 Form factor of a very long cylinder . . . . . . . . . . . . . 173

B.4 Estimating the SAXS signal for octamers and hexadecamers . . . . . 173

C MatLab code for calculating SAXS form factors $\quad 175$

$\begin{array}{ll}\text { D Code for evaluating COMSOL 4.0a simulations } & 179\end{array}$

D.1 Calculating streamlines . . . . . . . . . . . . . . . . . . 179

D.2 Evaluating the simulation along the streamline . . . . . . . . . 181

D.3 Integration along the streamlines . . . . . . . . . . . . 185

D.4 Determination of ensembles . . . . . . . . . . . . . . . 188 
E Principal component analysis

List of abbreviations

List of Figures

198

Bibliography

205 


\section{Chapter 1}

\section{Introduction}

When we touch our skin, the cells in it are able to maintain their mechanical integrity and deform to accommodate the stress. Structural stability and adaptability of cells are vital for the sustenance of any living organism. In eukariotic cells, an interacting network of proteins forms a flexible scaffold which determines their mechanical properties, the cytoskeleton. Its most prominent constituents are three different systems of biopolymers: Microtubules, which serve as tracks for intracellular tranport, actin filaments, which are key players in cell motility, and intermediate filaments, which are cell-type specific and maintain cell shape. Some intermediate filaments are also found outside of living cells, like cross-linked keratins in hair and nails [1].

Mutations in cytoskeletal proteins can cause severe diseases. For example, certain mutations in the intermediate filament desmin result in cardiomyopathy which can cause cardiac arrhythmias [2]. Mutations in the intermediate filament NF-M have been linked to the onset of Parkinson's disease [3]. This work will focus on the intermediate filament vimentin, for which mutations which prevent correct filament formation, cause a dominant, pulverulent cataract [4].

The cytoskeleton is a very dynamic structure which constantly reforms itself $[5,6]$. Complex regulation networks, including protein expression and protein interactions, govern these processes [7]. For example, the formation of cytoskeletal filament networks in vivo depends on the interaction of their protein precursor which each other, but also on their interaction with other proteins, the (regulated) expression of the pre-cursor protein, local concentrations of molecules, etc., which themselves depend on other cellular processes. Due to this high degree of complexity it is difficult to determine underlying interaction principles of filament and filament network formation in vivo. By isolating components and studying them in vitro, specific interactions can be understood. 
Controlled in vitro conditions that mimic individual in vivo conditions can be achieved by different micro- and nanomanipulation methods. Techniques like optical tweezers and atomic force microscopes allow to apply precise mechanical stresses [8], microfluidics enable well defined local concentration control $[9,10]$ or can model crowding [11].

This thesis investigates how the assembly of intermediate filaments depends on the ionic composition of their environment. Towards this goal, we study how both the final structure and the structure of assembly intermediates of the intermediate filament vimentin and of one of its mutants changes in dependence of the concentration of different ions. Non-equilibrium states are prepared and studied in microflow. In chapter 2, the intermediate filament vimentin is introduced. Chapter 3 presents small angle X-ray scattering (SAXS) as a probe to macromolecular structure and chapter 4 discusses what we can learn by studying the effect of ions on the structure of filaments by SAXS. Chapter 5 explains how we can use microfluidics to study the formation of filaments in situ with SAXS. Theoretical considerations on the experiments are given in chapter 6 and chapter 7 presents our experimental results on the salt concentration dependence of the structure of assembly intermediates. In chapter 8 our findings are discussed in a broader (bio)physical context and chapter 9 summarizes our findings. 


\section{Chapter 2}

\section{Intermediate filament formation - a self-assembly process}

This chapter introduces the biopolymer family of intermediate filaments as a part of the cytoskeleton and their assembly in vitro as a self-assembly process. It describes our current understanding of the molecular properties of vimentin, a common intermediate filament, and the biochemical methods for the preparation of vimentin for in vitro experiments.

\subsection{Vimentin, an intermediate filament protein}

The shape of cells can vary considerably from cell type to cell type [12], e.g. starfish shaped astrocytes or toroidal red blood cells. In animal cells, the form of a cell is to a large extent determined by its cytoskeleton, a complex networks of both filamentous and non-filamentous proteins $[13,14]$. In metazoan cells, three separated filamentous components exists: Actin filaments (also: microfilaments), microtubules and intermediate filaments.

Actin and microtubules are polar filaments with associated directional motor proteins. In contrast, intermediate filaments are non-polar and no associated motor proteins are known [1]. Whereas the precursor proteins of actin filaments and microtubules are highly conserved, the precursor proteins of intermediate filaments vary from cell type to cell type with more than 65 different encoding genes identified in the human genome by 2004 [18]. Interestingly, some cells possess more than one distinct cytoskeletal intermediate filament network, e.g. both a keratin and a vimentin network [19]. 
(a)

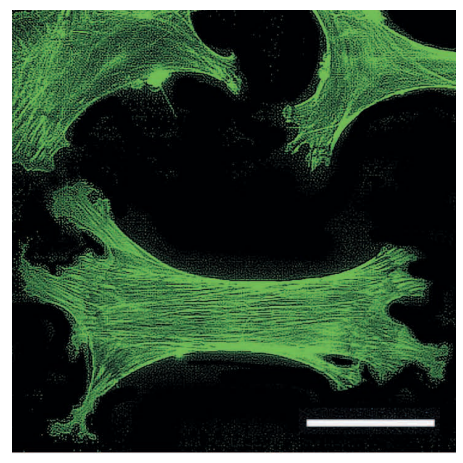

(b)

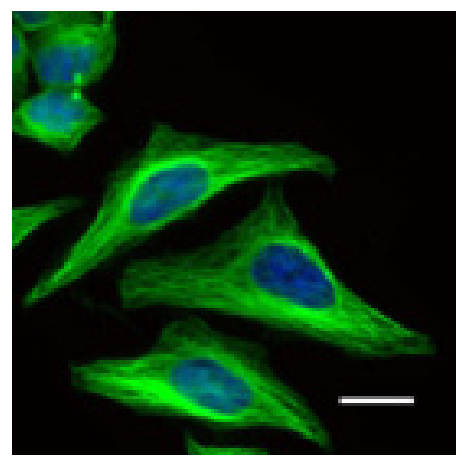

(c)

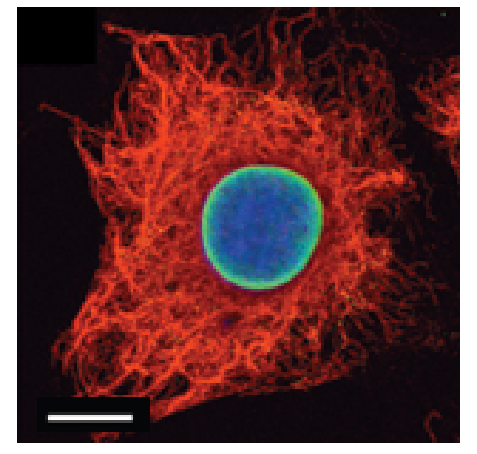

Figure 2.1: Cells stained for the three main filamentous components of the cytoskeleton. (a) Lung fibroblasts stained green for F-actin. The scale bar represents $50 \mu \mathrm{m}$, from [15]. (b) HeLa cells stained for microtubules (green) and DNA (blue). The scale bar represents $10 \mu \mathrm{m}$, from [16]. (c) The cytoplasmic vimentin IF network (red) and the lamin IF network (green) in the nucleus (DNA in blue) of a higher metazoan cell. The scale bar represents $10 \mu \mathrm{m}$, from [17].

$\begin{array}{lll}\text { Type I } & \text { acidic keratins } & \text { form heterodimers with type II } \\ \text { Type II } & \text { neutral and basic keratins } & \text { form heterodimers witch type I } \\ \text { Type III } & \begin{array}{l}\text { vimentin } \\ \text { desmin }\end{array} & \begin{array}{l}\text { form homodimers } \text { in vivo } \\ \text { can form heterodimers }\end{array} \\ & \text { GFAP } & \begin{array}{l}\text { with other type III or IV IF } \\ \text { can form heterodimers } \\ \text { with other type III or IV IF }\end{array}\end{array}$

Table 2.1: Main classes of cytoskeletal intermediate filaments, adapted from [20]

In general, most cytoskeletal intermediate filaments belong to one of several main classes which are based on primary structure, gene structure, assembly properties and tissue-specific expression patterns [20]. An overview of the most important classes with some examples is given in table 2.1. Intermediate filament networks are remarkably stable against solubilisation, remaining insoluble even at conditions that solubilize actin filaments and microtubules, such as treatment with surfactants, e.g. Triton X-100 [21,22].

\subsubsection{Vimentin}

Vimentin is a type III intermediate filament and naturally occurs in cells of mesynchymal origin. Recently, Mendez et. al. reported that the presence of vimentin filaments in cells is sufficient to alter their shape and motility from epithelial to mesenchymal [12]. In vitro, vimentin filaments can self-assemble from tetrameric pre-cursor proteins in a hierarchical way, including lateral assembly to unit length 
filaments (ULFs) and compaction of early filaments to mature filaments [23].

\section{Structure and mechanical properties of vimentin}

The smallest subunit of vimentin is monomeric vimentin. It is, however, only found at high urea concentrations above $6 \mathrm{M}$ [24]. The next larger subunit is the dimer, that is predominant at e.g. $3 \mathrm{M}$ guanidimium hydrochloride [25]. The monomers form an $\alpha$-helical coiled coil rod domain flanked by flexible tail and head domains (figure 2.2), which is a common structure motive of intermediate filament proteins [26]. For human vimentin, 334 amino acids contribute to the rod domain, 77 to the head and 55 to the tail [27]. The structure of the rod domain has recently been determined at atomic resolution by crystallography [28, 29].

Based on these structures of the dimer rod domain, Sokolova et. al. modeled

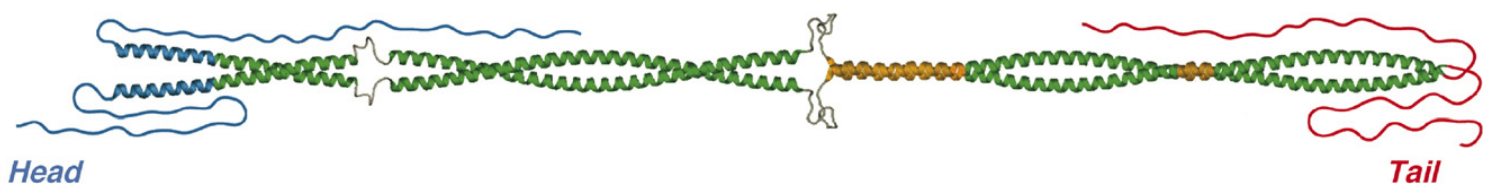

Figure 2.2: The basic structure of the vimentin dimer. An $\alpha$-helical coiled-coil rod domain is flanked by flexible head and tail domains. The atomic structure of the roddomain is solved [29]. Figure adapted from [26].

oligomers (tetramers, octamers and 32-mers (ULFs)) to fit corresponding small angle $\mathrm{X}$-ray scattering data. The optimal distance of the dimers in the tetramers was

(a)
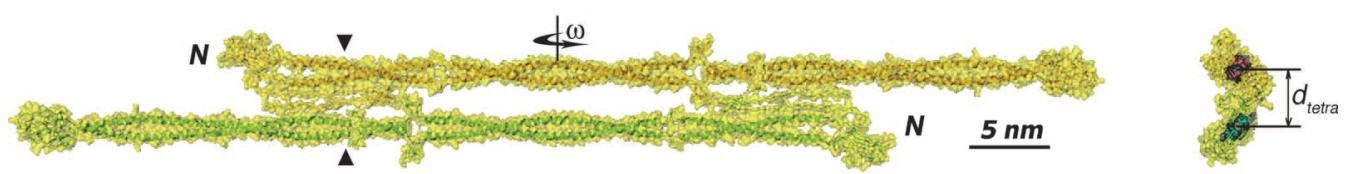

(b)
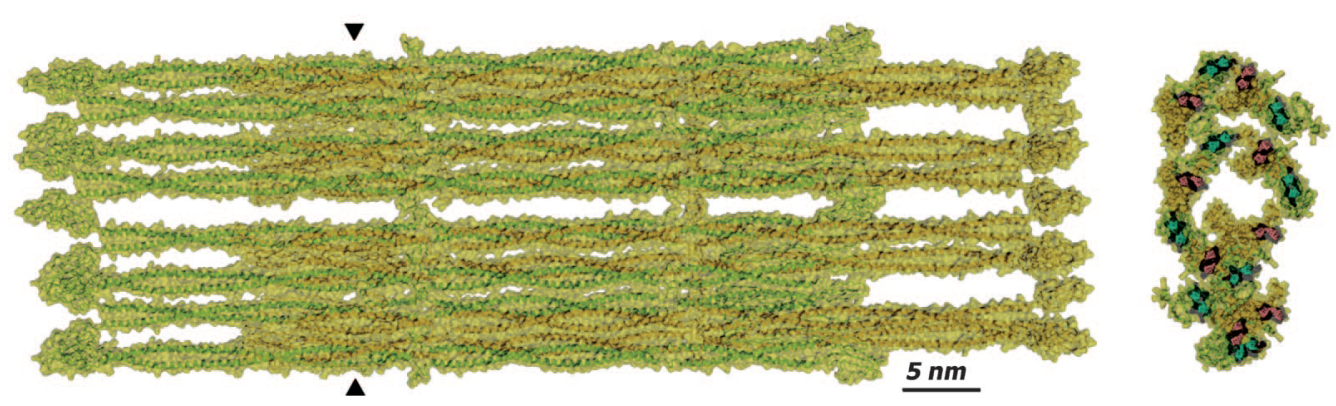

Figure 2.3: Models of the structure of (a) vimentin tetramers and (b) ULF. The left hand of the figure shows the side views, the right side the cross-sectional views. Both models are based on the optimazation of dimer locations to fit to SAXS curves. From [30]. 
determined to $3.4 \mathrm{~nm}$ (figure 2.3a). The resulting cross-section for the ULF was an ellipsoidal barrel with axis lengths of $16 \mathrm{~nm}$ and $9 \mathrm{~nm}$, which is slightly less then the value reported from transmission electron microscopy (TEM), $17 \mathrm{~nm}$ (figure 2.3b). The length of both tetramers and ULFs is about $60 \mathrm{~nm}[30,31]$. The diameter of mature vimentin filaments is $9.5 \pm 1.1 \mathrm{~nm}$ by TEM, their length can reach up to several micrometers and their persistence length is $2 \mu \mathrm{m}[11,32]$. Based on electron micrographs of neurofilaments where the side chains are clearly visible as protrusions from the central filament, the tail chains for all IFs are assumed to be located around the filament [33].

\section{Interaction of IFs with ions in vitro}

The addition of monovalent ions $\left(\mathrm{K}^{+}, \mathrm{Na}^{+}\right)$initiates the self-assembly to extended filaments (diameter $10 \mathrm{~nm}$, length up to several $\mu \mathrm{m}$ ), involving several distinct substeps $[27,34-36]$. In contrast, the effect of the addition of di-valent ions is more complex. Hofmann et al. report that the addition of $5 \mathrm{mM} \mathrm{CaCl}_{2}$ to tetrameric vimentin leads to the formation of thick ( $15 \mathrm{~nm}$ to $40 \mathrm{~nm}$ diameter for recombinant amphibian vimentin) and comparatively short filaments [36], while the addition of magnesium or lower calcium concentrations leads to seemingly normal filaments. However, Kooijman et al. report that vimentin forms small oligomers (dimers to octamers) at $0.3 \mathrm{mM} \mathrm{MgCl}_{2}, \mathrm{pH} 7.5$ which grow to longer protofilaments when the magnesium concentration is increased up to $2.4 \mathrm{mM}$ [37].

In addition, in the presence of monovalent ions both magnesium and calcium ions induce identical cross linking of the networks as indicated by rheology, and the structure of the filaments appears identical to filaments formed in the presence of only monovalent ions [38-40]. Related to that, Dammann at al. showed that at magnesium to vimentin ratios in the range of 2600 the vimentin networks start to strongly compact [41]. Further, Stromer et al. reported that desmin filaments formed by adding either calcium or magnesium ions resemble each other and are distinctively thicker than those formed by the addition of sodium or both sodium and magnesium. The results by Stromer further indicate, that these differences are not only due to changes in the ionic strength of the buffer, but also to differences of the interaction of mono- and di-valent ions with the protein [42]. At pre-elongation stages of vimentin at less than $0.5 \mathrm{mg} / \mathrm{ml}$, analytical ultracentrifugation indicates that increasing potassium concentration from $0 \mathrm{mM}$ to $20 \mathrm{mM}$ leads to larger oligomers with a broader size distribution [43].

A further factor to consider is the choice of buffer system. For studies of assembly 
kinetics, mostly a relatively weak "phosphate" buffer $(2 \mathrm{mM}$ phosphate plus varying salt contents) has been chosen. Most structural studies were performed in a stronger "TRIS" buffer system $[30,36,44]$. The advantage of using the "phosphate" system for kinetic studies is that for the initiation of filament assembly only the monovalent ion concentration needs to be increased, whereas in the "TRIS" system usually the buffer concentration, salt concentration and $\mathrm{pH}$ value are changed simultaneously. Tetramers and filaments in both buffer systems are identical, but Winheim et al. found that filaments present in "phosphate" buffer after $10 \mathrm{~s}$ of assembly at $0.2 \mathrm{mg} / \mathrm{ml}$ protein, i.e. pre-compaction, are considerably thinner (12 $\mathrm{nm})$ than those in "TRIS" buffer (17 nm) [44].

Interestingly, a single point mutation in the rod domain of vimentin is sufficient to hinder the elongation process. In the mutant $Y 117 \mathrm{~L}$ the tyrosine amino acid at position 117 of the protein is replaced by a leucine amino acid. This stabilizes the coiled-coil of dimeric vimentin but effectively stops the assembly process of the stage of ULFs $[43,45-47]$.

The influence of cation concentration on the size of the oligomers at less than $0.5 \mathrm{mg} / \mathrm{ml}$ appears complex: From $0 \mathrm{mM}$ to $20 \mathrm{mM}$ potassium chloride the oligomers become larger but in contrast to the wild type, the heterogenity increases only mildly and intermediate states seem to exist. Direct comparison with the wild type indicates that the size distribution of both species at $10 \mathrm{mM} \mathrm{KCl}$ differs. At higher salt concentrations, the diameter of the ULF-like structures formed by the mutant increases from $11 \mathrm{~nm}$ to $22 \mathrm{~nm}$ as the potassium chloride concentration increases from $30 \mathrm{mM}$ to $160 \mathrm{mM}$ in "phosphate" buffer, with slighlty larger values in the "TRIS" buffer system [43].

\subsubsection{Kinetics of vimentin assembly in vitro}

In contrast to actin filaments or microtubules which grow by addition of single sub-units to one growing end using ATP or GTP, respectively, and which display a distinct lag phase, vimentin assembly is a hierarchical multi-step process which starts quasi-instantaneously upon the addition of monovalent ions $[1,35]$.

TEM and atomic force microscopy (AFM) of assembly snapshots revealed that at $0.1 \mathrm{mg} / \mathrm{ml}$ vimentin lateral assembly intermediates, the unit lengths filaments (ULFs), consisting on average of eight tetramers, are the predominant species $10 \mathrm{~s}$ after the initiation of the assembly. The critical vimentin concentration for this step appears to be less than $0.005 \mathrm{mg} / \mathrm{ml}$ (about $25 \mathrm{nM}$ tetrameric vimentin) [27]. The rate constant of lateral assembly from tetramers to octamers, from octamers to hex- 


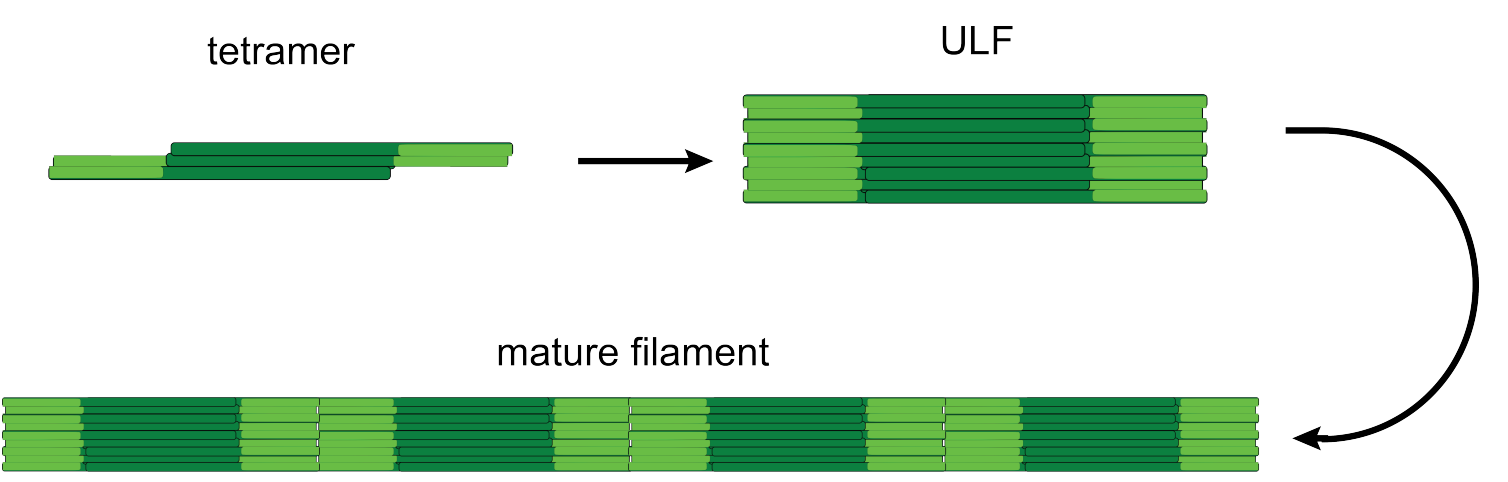

Figure 2.4: Upon addition of monovalent ions, vimentin tetramers first laterally assemble to unit length filaments (ULFs). The ULFs then align to form filaments. Finally, the filament radially compacts from about $17 \mathrm{~nm}$ to about $10 \mathrm{~nm}$ [35]. Sketches courtesy of B. Nöding.

adecamers and from hexadecamers to ULFs could be determined to be in the range of $20 \cdot 10^{6} \mathrm{M}^{-1} \mathrm{~S}^{-1}[23,27,35]$. Smaller intermediates than the ULF have not yet been directly identified for wild type vimentin, but for the K139C mutant conditions with predominantly octameric vimentin could be identified [30].

The formation of ULFs is followed by the longitudinal assembly of ULFs to filaments and annealing of single filaments. With a rate constant of $1.7 \cdot 10^{6} \mathrm{M}^{-1} \mathrm{~s}^{-1}$ this process is considerably slower, and continues up to days $[44,48]$. Finally, the filaments radially compact from about $17 \mathrm{~nm}$ diameter to about $10 \mathrm{~nm}$ diameter maintaining their mass per length $[27,46,47]$.

\section{$2.2 \quad$ Self-assembly processes}

Besides being of interest from a biological point of view, intermediate filemants also offer an interesting system for super molecular self-assembly [49]. Self-assembly typically refers to the well defined formation of larger structures from individual building blocks, such as the in vitro reconstitution of viruses from their isolated components [50], the organization of amphiphilic molecules in micelles [51] or the association of protein-protein complexes [52]. What distinguishes self-assembly from other assembly processes is the lack of an external 'building plan', i.e. the emerg- 
ing structure results from the intrinsic properties of its constituents. A further, somewhat vague, criterium is the desirability of the structure. For example, the formation of amyloid fibrils is in general not considered as self-assembly, despite having a well-defined structure based on intrinsic properties of the involved proteins [53].

For cells, large structures based on self-assembly of repetitive sub-units minimize the amount of structural information that needs to be stored in the genome, reduce the effect of transcription errors and allow for recycling of sub-components $[5,7]$.

Self-assembly is an intermolecular process, i.e. no covalent bonds are formed between the components. Instead, the hydrophobic effect, hydrogen bonds, electrostatic interactions and van der Waals interactions generate a large enough net force to hold the assembly together. Therefore, electrostatic screening by ions in the solution can drastically affect the assembly process [54]. Because the structure of vimentin filaments changes with the ionic composition of the buffer (2.1.1) and meta-stable intermediate states have been identified, a systematic study of the vimentin assembly at different ion concentrations can broaden our understanding of the interactions underlying self-assembly processes.

\subsection{Reconstitution of vimentin}

Scattering experiments for structure determination require highly purified protein samples at concentrations in the range of $\mathrm{mg} / \mathrm{ml}$ to obtain adequate signal to noise ratios [55]. The expression of vimentin in E.coli bacteria provides both a high protein yield and good purity, rendering it the expression system of choice [56].

Briefly, E.coli bacteria are transfected with a plasmid encoding the desired vimentin protein (human wild type or $Y 117 \mathrm{~L}$ mutant) and cultivated to express the protein. The protein is purified from the bacterial solution by several centrifugation, homogenization and washing steps in different buffers and two ion exchange chromatography columns, leading to typical concentrations in the range of $3-4 \mathrm{mg} / \mathrm{ml}$. The purity of the vimentin is controlled by an SDS page gel. The purified vimentin can be stored in urea solution at $-80^{\circ} \mathrm{C}$ for several months. Details of these steps are given in appendix A.

For assembly experiments, the vimentin needs to be transferred to an adequate ureafree buffer system (TRIS buffer or phosphate buffer, see 2.1.1). This is achieved by step-wise dialysis at decreasing urea concentrations ( $8 \mathrm{M}, 4 \mathrm{M}, 2 \mathrm{M}, 1 \mathrm{M})$ and increasing dialysis buffer content $(20 \%, 60 \%, 80 \%, 90 \%) 30 \mathrm{~min}$ each and two steps in of $2 \mathrm{~h}$ and $12 \mathrm{~h}$ in dialysis buffer, in a dialysis membrane with an upper 
permeability limit of $50 \mathrm{kDa}$ which is less than the monomer weight of $54 \mathrm{kDa}$. The actual resulting protein concentration is determined by UV-spectroscopy, see below. Lower protein concentrations are achieved by dilution with dialysis buffer, higher concentrations by centrifugation in Nanosep 10K centrifuge concentrators (Nanosep Centrifugal Devices, PALL, Ann Arbor, Michigan, USA) at $13.4 \mathrm{rpm}$ in a $6 \mathrm{~cm}$ rotor (MiniSpin, Eppendorf, Hamburg, Germany). The dialysed vimentin can be stored for up to a week at $4{ }^{\circ} \mathrm{C}$.

\section{Determination of the protein concentration}

For comparison of SAXS curves and interpretation of reaction kinetics, knowledge of the actual protein content of a sample is required. A straightforward way to determine the concentration of a given protein in solution is the measurement of the absorbance $A=\log \left(I / I_{0}\right)=\epsilon l_{s} c$ where $\epsilon$ is the protein specific extinction coefficient at $280 \mathrm{~nm}, l_{s}$ the optical path length and $c$ the protein concentration [57].

For the human vimentin wild type $\epsilon$ is about $24 \cdot 10^{3} \mathrm{M}^{-1} \mathrm{~cm}^{-1}$ [37]. However, the precise extinction coefficient depends on the protein environment (buffer type, ions, denaturants), which adds to the measurement error. For the Y117 $\mathrm{L}$ mutant, the extinction coefficient can be estimated in the amino acid sequence to about $5.7 \%$ less than the extinction coefficient of the wild type. This shows that the absolute concentrations of proteins can only be approximates, but for one vimentin type in one buffer systems the relative concentration between different samples can be obtained. The absorbance of the protein samples was determined with a Nanodrop Spectrometer (Nanodrop ND-1000, ThermoScientific Technologies, Inc.,Wilmington, USA) which requires a sample volume of only $2 \mu$ l. Variation in the optical path length and the incident intensity add to an intrinsic error for a single measurement of about $10 \%$. Therefore, our protein concentrations are always the mean of at least three measurements. 


\section{Chapter 3}

\section{Small angle X-ray scattering - a probe for macromolecular structure}

This chapter introduces small angle X-ray scattering (SAXS) as a tool for the investigation of macromolecular structure and structural changes. It explains general and technical aspects of SAXS experiments and their analysis.

\subsection{Advantages and applications of SAXS}

In order to investigate changes in the conformation of vimentin in different ionic conditions we require a probe which is sensitive to structural changes on the appropriate length scales, i.e. from about $1 \mathrm{~nm}$ up to $100 \mathrm{~nm}$. Additionally, the technique needs to be compatible with heterogeneous samples. Finally, to avoid artifacts from sample preparation and to observe changes in the structure in situ, investigations of samples in solution are needed. SAXS is a technique that fulfills all these requirements [58]. In a basic SAXS experiment, an X-ray beam hits the sample and is scattered. At a distance $L_{D}$ from the sample, the intensity of the scattered light is recorded in dependence of the angles $\theta$ and $\phi$ (fig. 3.1). $\theta$ and $\phi$ for a photon depend on its momentum transfer in the sample, also called the "scattering vector", $\vec{q}=\vec{k}^{\prime}-\vec{k}$, where $\vec{k}$ and $\vec{k}^{\prime}$ are the momentum before and after scattering, respectively. It is a straightforward geometric argument that for elastic scattering, i.e. $\left|\vec{k}^{\prime}\right|=|\vec{k}|$

$$
|\vec{q}|=\left|\vec{k}^{\prime}-\vec{k}\right|=q=\frac{4 \pi}{\lambda} \sin (\theta / 2)
$$

where $\lambda$ is the wavelength of the photon and that $\phi$ corresponds to the angle of $\vec{q}$ in the detector plane. Because typical SAXS experiments average over orientation and 


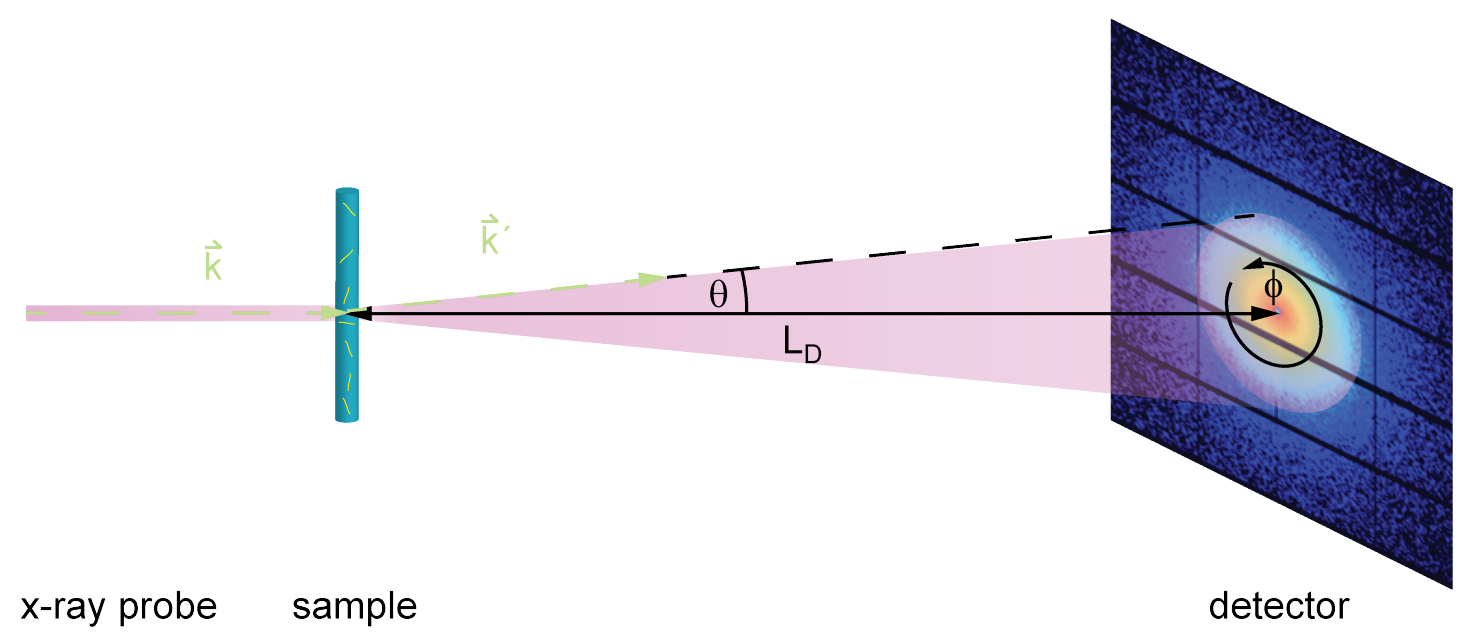

Figure 3.1: In a SAXS experiment, an X-ray beam is scattered by the sample. The intensity of the scattered light is recorded at a distance $L_{D}$ in dependence of the angles $\theta$ and $\phi$. To resolve sufficiently small $\theta, L_{D}$ is typically in the range of meters.

polydispersity of a soluted sample, the information content of a scattering pattern is low in comparison to crystallography. Therefore, SAXS is often referred to as a low resolution technique, although it is very sensitive to structure in the nanometer range $[55,59]$.

SAXS is especially sensitive to the general shape of a macromolecule and its changes. Therefore it is a powerful tool to study how proteins fold from molten globules to a well defined structure [60,61], how proteins arrange in complexes or oligomers $[30,62]$, how polymers deform under stress [63] or the supramolecular structure of large, polydisperse complexes [64]. At high protein concentrations (typically above $10 \mathrm{mg} / \mathrm{ml}$ ) SAXS can even access the protein-protein interaction potential [65]. Due to the fact that SAXS experiments can be performed in solution, dynamic processes can be investigated without the intrinsic limitation to static snap-shots.

Recent progresses in sources and optics for X-rays have led to weakly divergent microbeams with a high intensity which enable spatially resolved SAXS experiments in heterogeneous samples [66]. This allows for novel applications like the combination of SAXS with microfluidics as discussed in chapter 5. 


\subsection{General considerations}

In order to obtain relevant information from the SAXS signal of a macromolecule, it is necessary to understand what determines the signal. In general, the scattering intensity $I(\vec{q})$ of a set of $N$ atoms in dependence of the scattering vector $\vec{q}$ is given by

$$
I(\vec{q}) \propto \sum_{i=1}^{N} \sum_{j=1}^{N} b_{i} b_{j}^{*} e^{i \vec{q}\left(\vec{r}_{i}-\vec{r}_{j}\right)}
$$

where $r_{i}$ is the location of the $i$ th atom and $b_{i}$ its 'scattering' length $[67,68]$.

For a set of discrete scattering objects, e.g. molecules, eq. 3.2 can be split up into the intramolecular form factor $F$ and the intermolecular structure factor $S$ [67-69]:

$$
I(\vec{q}) \propto S(\vec{q}) F(\vec{q})
$$

The form factor for a scattering object with an electron density $\rho(\vec{r})$ is

$$
F(\vec{q})=\left|\int_{V} \rho(\vec{r}) \exp (i \vec{q} \vec{r}) \mathrm{d}^{3} r\right|^{2}=\int_{V} \gamma(\vec{r}) \exp (i \vec{q} \vec{r}) \mathrm{d}^{3} r
$$

where $\gamma(\vec{r})=(\rho \otimes \rho)(\vec{r})$ is the autocorrelation of its electron density and the last identity follows from the convolution theorem. The structure factor describes the interference caused by the correlation of different molecules in the solution and depends, amongst others, on the concentration and molecule interactions, such as repulsion.

When the orientation of molecules in the sample is isotropic, the scattering becomes independent of the angle $\phi$ of $\vec{q}$ and is given by

$$
I(q)=\langle S(\vec{q}) F(\vec{q})\rangle
$$

where $\langle\cdots\rangle$ denotes spatial averaging over all possible molecule orientations or, equivalently, all possible orientations of $\vec{q}$. At low concentrations, the scatterers are usually randomly distributed, and the structure factor is therefore very close to unity $[67,70]$, i.e. $S(\vec{q})=S(q) \approx 1$. Therefor the intensity depends only on the form factor:

$$
I(q)=\langle F(\vec{q})\rangle=\left\langle\int_{V} \gamma(\vec{r}) \exp (i \vec{q} \vec{r}) \mathrm{d}^{3} r\right\rangle=\int_{0}^{D} 4 \pi p(r) \frac{\sin (q r)}{q r} \mathrm{~d} r
$$


where $D$ is the maximum point-to-point distance in the molecule and $p(r)=\langle\gamma(\vec{r})\rangle r^{2}$ the pair distance distribution function (PDDF). A preferred orientation of molecules in the sample, e.g. due to flow, leads to non-centrosymmetric scattering patterns [71].

For long, extended molecules like intermediate filaments, the scattering amplitude can in general be separated into two factors corresponding to the longitudinal contribution $F_{L}$, depending on the length $L$ of the molecule, and the cross-sectional contribution $F_{C}[69]$ :

$$
I(q)=L^{2} F_{L} \cdot F_{c}
$$

The longitudinal contribution is given by the form factor for an infinitely thin rod (Neugebauer form factor) [72]

$$
F_{L}(q)=\int_{0}^{\infty}\left(\frac{\sin (q L \zeta / 2)}{(q L \zeta / 2)}\right)^{2} d \zeta=2 \frac{\operatorname{Si}(q L)}{q L}-\left(\frac{\sin (q L / 2)}{q L / 2}\right)^{2}
$$

introducing the sine integral

$$
\operatorname{Si}(x)=\int_{0}^{x} \frac{\sin t}{t} \mathrm{~d} t
$$

The two-dimensional form factor of the cross-section is given by

$$
F_{c}=\left|\int_{A} \rho\left(\vec{r}_{c}\right) \exp \left(i \vec{q} \vec{r}_{c}\right) \mathrm{d}^{2} r_{c}\right|^{2}
$$

For $q L \gg 1, F_{L}$ can be further simplified to

$$
F_{L}(q)=\frac{\pi}{q L}
$$

It follows that the scattering of filaments does not level of at small $q$, but instead display a $q^{-1}$ slope. Further, eq. 3.10 implies that the scattering from long filaments is proportional to their length. Longitudinal form factors for semi-flexible polymers with sufficiently long persistence length show a similar $q$-dependence for small $q$ $[69,73]$.

Analogously to eq. 3.6, orientational averaging of $F_{c}(\vec{q})$ gives

$$
F_{c}(q)=\int_{0}^{D} 2 \pi p_{c}(r) B_{0}(q r) \mathrm{d} r
$$


where $B_{0}$ is the Bessel function of zero order and the average cross-sectional pair distance distribution function is defined as $p_{c}(r)=r\left(\rho_{c} \otimes \rho_{c}\right)(r)$.

For polydisperse solutions consisting of $N$ species with neglegible intermolecular scattering, the total scattering intensity is given by

$$
I(\vec{q})=\sum_{i=1}^{N} c_{i} F_{i}(\vec{q})
$$

where $c_{i}$ is the concentration of the $i$ th species and $F_{i}$ its form factor. From eq. 3.10 it follows that a solution of filaments of varying lengths leads, for sufficiently long filaments, to the same scattering as a solution of filaments of the mean length only. However, when the different species differ on length scales directly accessible by the SAXS experiment, the overall scattering curve in general does not correspond to the scattering curve of the mean structure. Further, it is important to note that polydispersity and anisotropy of a sample often cannot be distinguished and therefore preliminary information on either one is often required for data analysis [74].

\subsection{Acquisition of SAXS data}

In this section, the different SAXS set-ups used for different experiments will be illustrated and the initial data treatment will be discussed.

\subsubsection{Steady state experiments at DORIS III - B1}

The equilibrium states of vimentin at different ion and protein concentrations, i.e. the states reached after several hours at these conditions, are investigated by steady state SAXS experiments. For these experiments, the vimentin assembly is initiated directly after dialysis by addition of concentrated salt buffer to obtain the desired salt and protein concentration and the solution is filled into capillaries (mark-tubes made of quartz glass, outer diameter $1.5 \mathrm{~mm}$, wall thickness $0.01 \mathrm{~mm}$; Hilgenberg, Malsfeld, Germany) right after mixing. The filled capillaries are accelerated to 1500 rpm (Eppendorf centrifuge 5810 R, Eppendorf, Hamburg, Germany) and instantaneously decelerated again to remove air bubbles in the solution. Samples are stored at $4{ }^{\circ} \mathrm{C}$ for at least $24 \mathrm{~h}$ before recording the SAXS signal. The SAXS signal is recorded at the beamline B1 at DORIS III (Hasylab, Hamburg, Germany), a low flux bending magnet beamline optimized for anomalous small an- 
gle X-ray scattering studies $[75,76]$. A sketch of the beamline layout is given in figure 3.2. B1 has no focusing optics, therefore the beam at the sample position

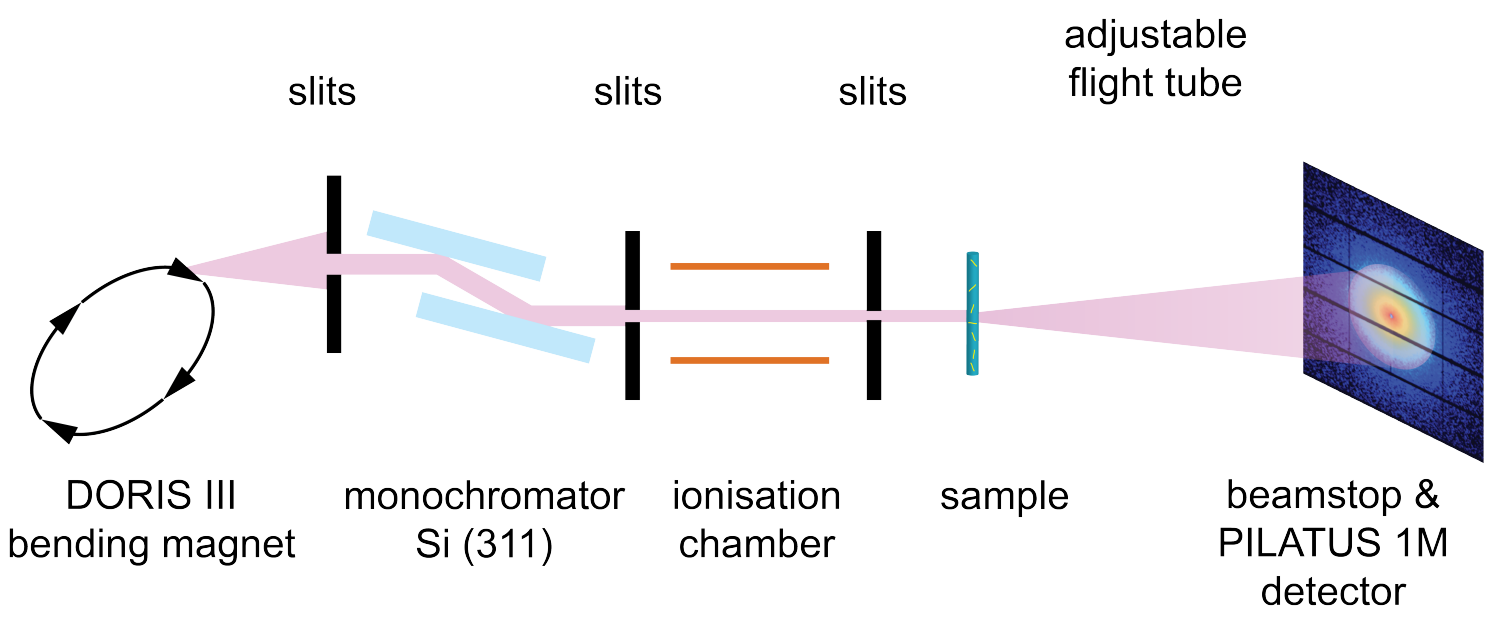

Figure 3.2: Main components of the beamline B1 at DORIS III at HASYLAB, Hamburg, Germany. B1 is located at a bending magnet. A pair of water cooled $\mathrm{Si}(311)$ crystals monochromizes the beam. The beam size is determined by several sets of slits without any focusing. Detection of beam intensity by the ionisation chamber and automated measurement of calibration standards give access to absolute scattering lengths. The detector to sample distance is easily changeable, providing access to an extended $q$-range. The semi-transparent beamstop allows precise detection of the centre of the beam. The entire setup is evacuated. The sketch is based on the beamline description given in [76].

is comparatively large and the photon count is low. However, an easily adjustable flight tube length (from $88 \mathrm{~cm}$ to $3.5 \mathrm{~m}$ ) gives access to a large $q$-range with a good signal to noise ratio even at larger $q$. Most experiments described in this thesis were performed at $9 \mathrm{keV}$ at which the highest intensities can be reached. For some samples, however, $14 \mathrm{keV}$ were used which is closer to the maximum of the emission spectrum of the bending magnet. The beamline is equipped with a PILATUS 1M detector $\left(981 \times 1043\right.$ pixels, pixel size: $172 \times 172 \mu \mathrm{m}^{2}$; Dectris, Baden, Switzerland).

All samples are measured at $3.5 \mathrm{~m}$ sample to detector distance for up to $1 \mathrm{~h}$ and at $88 \mathrm{~cm}$ for up to $40 \mathrm{~min}$. Exposure times are split into $5 \mathrm{~min}$ or $10 \mathrm{~min}$ intervals. The interval lengths and the total number of exposures depend mostly on the stability of the DORIS III operation. A set of eleven sample exposures is always preceded by an empty beam measurement of $200 \mathrm{~s}$ and a reference measurement of $60 \mathrm{~s}$. On-site MatLab tools are used for automated radial integration of single exposures, averaging of the exposures and merging of short and long sample to detector distance data. 


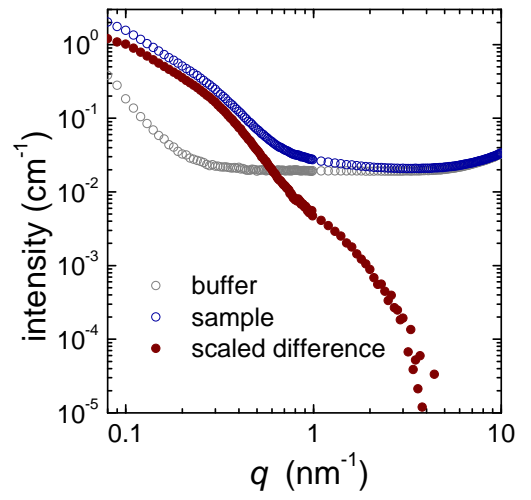

Figure 3.3: In order to correct for scattering from the buffer the scattering signal from a buffer capillary is scaled and subtracted from the signal of the vimentin sample.

To correct for background scattering from the buffer and the capillary itself, the SAXS curves of both the sample and the buffer are scaled to a mean of $1.64 \cdot 10^{-2} \mathrm{~cm}^{-1}$ between $q=3 \mathrm{~nm}^{-1}$ and $5 \mathrm{~nm}^{-1}$ and then subtracted from each other. This procedure assumes that in this range the main contributors to the scattering signal in this $q$-range are water and the capillary itself. The scaling is necessary because the precision of the absolute intensity scale is limited by the uncertainty of the capillary diameter which varies from capillary to capillary. An example of the original SAXS curves and the resulting background corrected curve are given in figure 3.3.

The increase in scattering towards $10 \mathrm{~nm}^{-1}$ is due to wide angle X-ray scattering (WAXS) from the capillary, buffer and the sample [77]. For an X-ray intensity of $14 \mathrm{keV}$ this procedure results in an accessible $q$-range with good statistics of $0.1 \mathrm{~nm}^{-1}$ to $3 \mathrm{~nm}^{-1}$, and for $9 \mathrm{keV}$ of $0.07 \mathrm{~nm}^{-1}$ to $3 \mathrm{~nm}^{-1}$.

\subsubsection{Microfluidic experiments at SLS - cSAXS}

SAXS experiments on microfluidic devices require X-ray spot sizes that are smaller than the channel width as scattering of the edges of the channels causes intense scattering. To maintain a sufficiently high photon flux high brilliance sources and focusing optics are required.

cSAXS at the Swiss Light Source (Paul-Scherrer Institut, Villigen, Switzerland) is a dedicated SAXS beamline equipped with mirrors to obtain spot sizes down to $5 \mu \mathrm{m}$ times $20 \mu \mathrm{m}$. A schematic of the beamline set-up is given in figure 3.4. cSAXS is an undulator beamline with focusing mirror optics. All experiments in this work are performed at $11.6 \mathrm{keV}$, a detector to sample distance of $7.17 \mathrm{~m}$, and a PILATUS $2 \mathrm{M}$ detector $\left(1475 \times 1679\right.$ pixels, pixel size: $172 \times 172 \mu \mathrm{m}^{2}$; SLS detector group, Villigen, Switzerland) resulting in a $q$-range of about $0.02 \mathrm{~nm}^{-1}$ to $2 \mathrm{~nm}^{-1}$. The beamline is equipped with a microscope which allows for precise determination of desired measurement spots. For this thesis, SAXS data of protein flow in microfluidic devices are recorded at cSAXS (see also chapters 5 and 7 ). The exposure time is set to three times $1 \mathrm{~s}$ for buffer background measurements and five 


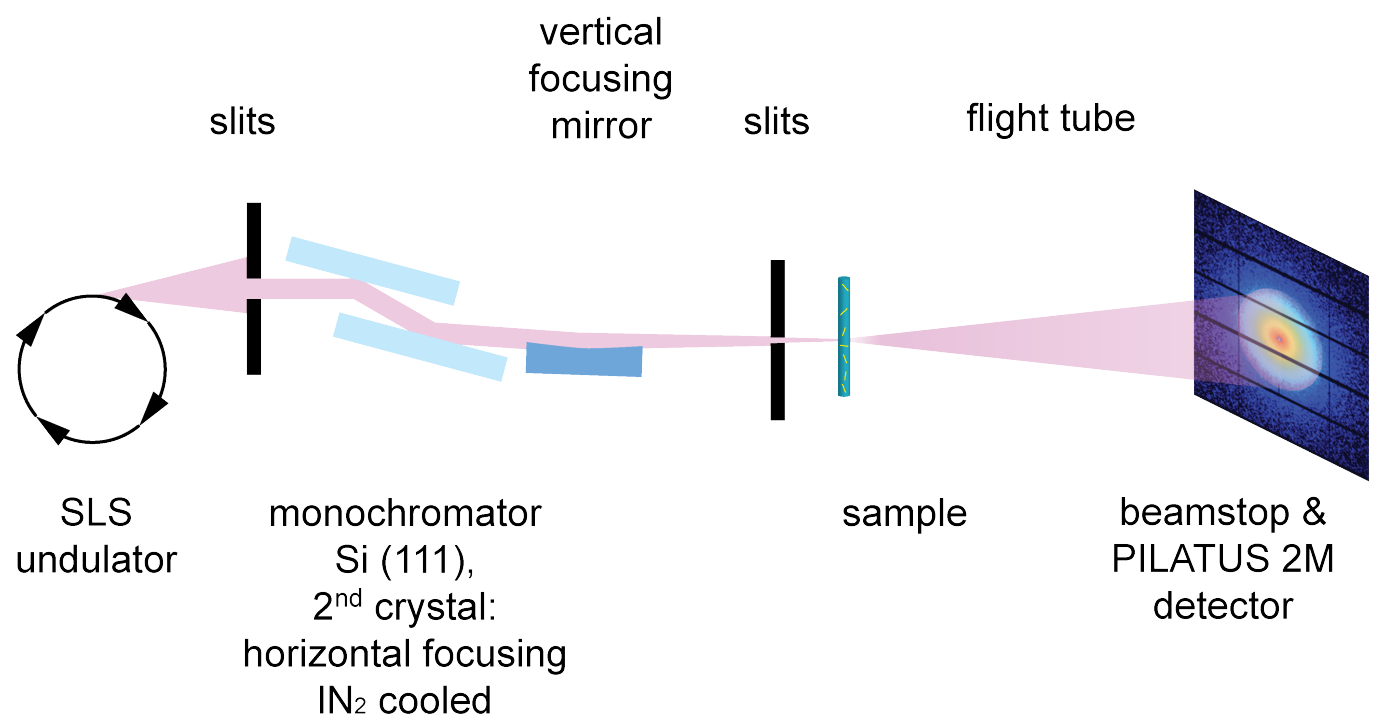

Figure 3.4: Main components of the beamline cSAXS at the SLS, Paul-Scherrer-Institut, Villigen, Switzerland. cSAXS is an undulator beamline. A pair of liquid nitrogen cooled $\mathrm{Si}(111)$ crystals monochromizes the beam. The beam size is determined by the combination of slits with a vertical focusing mirror and a horizontally focusing monochromator crytal. The PILATUS $2 \mathrm{M}$ detector is located at $7.2 \mathrm{~m}$ from the sample position. The path of the X-rays from the synchrotron to the sample position and from the sample position to the detector is evacuated. The sketch is based on the description of the beamline at [78].

times $1 \mathrm{~s}$ for protein flow. On-site tools are used for the radial integration of the data.

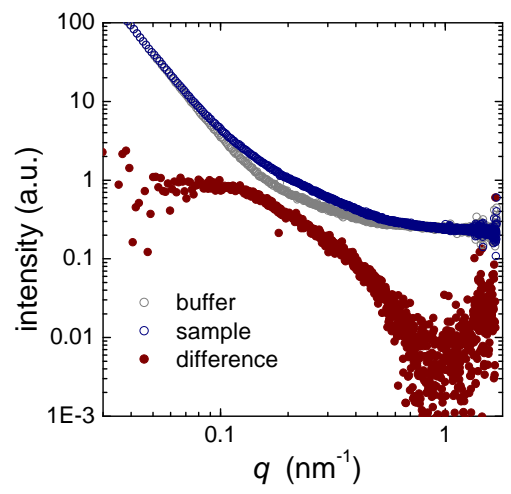

Figure 3.5: In order to correct for scattering from the buffer and the device, the scattering signal from a protein free measurement recorded on the same microfluidic device is subtracted from the signal of protein flow.
The background scattering in a microfluidic device depends on the amount of buffer and therefore the height of the microfluidic channel and on the thickness of the surrounding material. It therefore changes from device to device and also slightly depends on the position in a device. By recording SAXS signals for each device at suitable locations, appropriate backgrounds for each measurement are available and no additional scaling is necessary. Figure 3.5 gives an example for scattering curves of both proteinfree and protein-containing flow at the same location of the device, and their difference corresponds to the scattering of the protein. The contribution of the buffer and the device at small $q$ is considerably larger than for the capillaries 
and sets a lower level for the accessible $q$-range for experiments in flow at about $0.06 \mathrm{~nm}^{-1}$. No absolute scaling of the scattering intensity is available as no standard signal was recorded and no clear buffer signal without contributions from the device is accessible.

\subsection{Interpretation of SAXS curves}

As the scattering curves recorded in SAXS experiments always represent an ensemble of states the direct extraction of the macromolecular structure is in general not possible $[55,69]$. The determination of the radius of gyration based on the Guinier approximation offers a straightforward approach to the interpretation of the SAXS signal. However, the radius of gyration offers only a very rough description of a macromolecule and only determines the low- $q$ range of a SAXS curve.

Transformation of SAXS curves to real space provides the average pair distribution functions of the sample which are sensitive to symmetry and domain structure of the sample $[55,59]$. The most direct access to the sample structure is given by modeling approaches where a real-space model is adjusted to have its form factor fit the SAXS curve ('full $q$-range fit') [59, 79].

\subsubsection{Model-free analysis}

Model free approaches require little to no previous knowledge of the sample structure. Therefore, they can be applied to most systems and provide reliable results.

\section{Guinier approximation}

The Guinier approximation describes the small-q-limit of the form factor and was first derived by Guinier in 1939. The argument presented here follows [67]. For a globular particle we can approximate the form factor as

$$
\langle F(q)\rangle \propto \int_{0}^{D} p(r) \frac{\sin (q r)}{q r} \mathrm{~d} r \approx \int_{0}^{D} p(r)\left[1-\frac{(q r)^{2}}{6}\right] \mathrm{d} r
$$

by considering the first two terms of the Taylor series of the sine. As $\int_{0}^{D} p(r) \mathrm{d} r$ corresponds to the forward scattering length $I_{0}$ and $\frac{1}{2} \int_{0}^{D} p(r) r^{2} \mathrm{~d} r$ to the squared 
radius of gyration times the forward scattering length $R_{G}^{2} I_{0}$ per definition, we obtain

$$
\langle F(q)\rangle \approx I_{0}\left[1-\frac{\left(q R_{G}\right)^{2}}{3}\right] \approx I_{0} e^{-\frac{\left(q R_{G}\right)^{2}}{3}}
$$

For long rods, an analogous argument gives

$$
\langle F(q)\rangle \approx I_{0} \frac{e^{-\frac{\left(q R_{C}\right)^{2}}{2}}}{q}
$$

where $R_{C}$ is the radius of gyration of the cross-section. This second-order approximation usually holds for exponents smaller than 1, i.e. for $q R_{G}<\sqrt{3}$ for globular particles and $q R_{C}<\sqrt{2}$ for extended rods. For fitting purposes, it is therefore advisable to recursively optimize the fitted $q$-range. For a polydisperse mixture, the effective squared radius of gyration for the Guinier approximation follows from the first half of eq. 3.14 which shows it to be equal to the mean squared radius of gyration, i.e. $R_{G}^{2}=\sum_{i} f_{i} R_{G i}^{2}$ where $f_{i}$ is the fraction of the species $i$ and $R_{G i}$ its radius of gyration.

The radius of gyration $R_{C}$ and the forward scattering amplitude $I_{0}$ of the stationary data in the Guinier regime are determined by non-linear least square fitting to eq. 3.15 in MatLab R2009b (The MathWorks, Inc., Natrick, USA) using the lsqnonlin function of the optimization tool box. For samples with added salt, the $q$-range to which the fit is applied is recursively adjusted to fulfill the Guinier condition $\left(q R_{C}\right)^{2}=2$, the minimum $q$ is initially set to $0.1 \mathrm{~nm}^{-1}$ and decreased when both initial fits and visual conformation indicate the absence of finite length effects at smaller $q$. The initial maximum $q$ is $0.25 \mathrm{~nm}^{-1}$.

\section{Determination of pair distance distribution functions}

From equations 3.6 and 3.11, we see that the orientationally and ensemble averaged pair distance distribution functions (PDDFs) can be in principle obtained by inversion of the Fourier respectively Hankel transformations. However, the problem is ill-posed, i.e. small errors in the recorded intensity may result in large errors in the PDDF. Additionally, the finite size of the accessible $q$-range may induce termination effects $[80,81]$. Indirect Fourier transformation (IFT) addresses these problems by performing a least square fit of the data in $q$-space to the transform of a PDDF $p(r)=\sum_{\nu=1}^{N} c_{\nu} \phi_{\nu}(r)$ where $\phi_{\nu}$ are previously chosen basic functions, e.g. cubic splines [69]. From the sampling theorem, $p(r)$ is constrained to equal zero for $r>D_{\max }=\frac{\pi}{q_{\min }}$ where $q_{\min }$ is the smallest $q$ considered for the transformation and 
the maximal number of basic functions $N_{\max }=\frac{\Delta q D_{\max }}{\pi}$ where $\Delta q$ is the considered $q$-range [80].

We used the implementation of the IFT for long rods in the program GNOM (gnom45qw.exe) of the ATSAS 2.4 package provided by the 'Biological Small Angle X-ray Scattering' group at the European Molecular Biology Laboratory (EMBL), Hamburg, Germany [81,82]. The $q$-range for the first estimate was $\approx 0.21 \mathrm{~nm}^{-1}$ to $3.9 \mathrm{~nm}^{-1}$ and $D_{\max }$ was initially set to $30 \mathrm{~nm}$. Both $D_{\max }$ and the lower limit of the $q$-range are recursively adjusted such that the resulting PDDF is positive, approaches zero with a convex slope and no major oscillations occurred neither in the PDDF nor in the fit to the original data. In addition to the PDDF, GNOM provides an estimate of the forward scattering amplitude based on the fit to original data and the (cross-sectional) radius of gyration which can be estimated from the PDDF via

$$
R_{c}^{2}=\frac{\int_{0}^{D_{\max }} p(r) r^{2} \mathrm{~d} r}{2 \int_{0}^{D_{\max }} p(r) \mathrm{d} r}
$$

\subsubsection{Model-based analysis}

When sufficient data from other techniques (e.g. TEM, AFM) are available to build a model of the structure of the sample, model-based approaches to SAXS analysis give direct access to detailed structural information. Form factors for various basic structures have been reported and these can often be adapted to the sample of interest $[64,73,79]$. By attempting to fit the recorded data to the form factor structural models can be falsified and successful fitting can quantify structural parameters. Section 4.4.2 describes the structural models and corresponding form factors used in this work in detail. Fits to the different models were optimized by non-linear least square fitting in MatLab R2009b using the lsqnonlin function of the optimization

tool box on a $q$-range from $0.1 \mathrm{~nm}^{-1}$ to $3 \mathrm{~nm}^{-1}$. The implementation of the form factor in MatLab is given in appendix $\mathrm{C}$. 


\section{Chapter 4}

\section{The effect of cations on the equilib- rium state of vimentin}

In this chapter, we investigate how the structure of vimentin filaments depends on the assembly conditions. Towards this goal, we compare the SAXS signal of vimentin at different concentrations of precursor protein and different concentrations of monoand divalent ions and quantify the observed changes by both model-free and modelbased analysis. In addition, we also present data on the mutant $Y 117 \mathrm{~L}$, which forms stable ULFs but no filaments, at different protein and salt concentrations.

\subsection{Reproducibility of results}

Before comparing results for different assembly conditions, we need to control their reproducibility. Several factors need to be considered: Are the initial proteins identical? This is the case when the samples come from the same protein batch, but otherwise small differences can occur. Are the buffers used for assembly identical? Small differences in salt concentrations and $\mathrm{pH}$ cannot be avoided but might affect the structure. SAXS signals of samples are recorded during several days of instrument access ("beam time") and samples and buffers for one such beam time are usually prepared together and meet this criterion. Finally, the amount of time passed between sample preparation and measurement can affect structure of the filaments and their SAXS signal. For samples measured directly after each other this should be no major factor, but between the first and last sample measured during a beam time several days can pass. To understand to which extent we can compare results obtained within the same and during different beam times we there- 
fore need to compare results at nominally identical conditions recorded at different beamtimes as well as samples from different protein preparations. For tetrameric

(a)

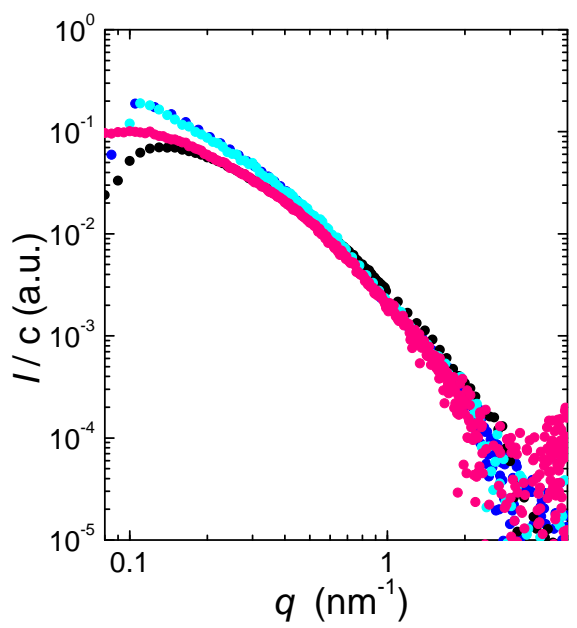

(b)

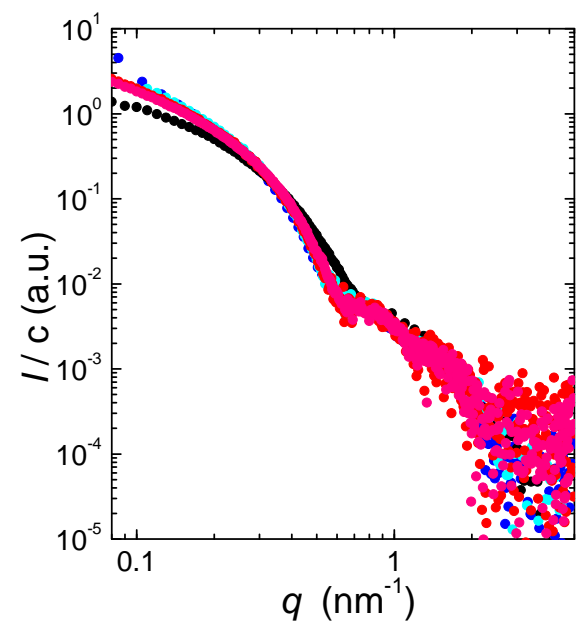

Figure 4.1: Comparison between the SAXS signal of nominally identical samples. Samples recorded at the same beam time have the same colour in both figures. (a) In the absence of additional ions (i.e. for tetrameric vimentin): The blue and cyan curves are from the same protein preparation and agree perfectly. The other curves are from different preparations and show small variations in the signal at small $q$. (b) At $80 \mathrm{mM} \mathrm{KCl}$ : The pink and red curves were recorded at the same beam time but with 4 days between them. The blue and cyan curves are again from the same preparation. Except for the black curve, all curves agree very well.

vimentin (i.e. no additional salt added to the sample), we see that the SAXS curves for one preparation agree perfectly. However, for different preparations the curves do not match at small $q$, indicating slight differences in the average structure, see figure 4.1a. These differences might be due to the sensitivity of tetrameric vimentin to the exact ionic environment, e.g. the exact composition of the buffer used in beamtime preparation. In the presence of $80 \mathrm{mM} \mathrm{KCl}$, most of the scattering curves match perfectly. This implies that even slightly different tetrameric samples lead to identical filament structures. The perfect agreement between the pink and red curves indicates that extended storage of 4 days does not alter the scattering signal. The black curve noticeably differs from the other curves, but the general shape and main features still agree, such as the existence of a kink and the general slope at small and large $q$.

We therefore can directly compare all results from one protein preparation independently on when they were recorded. Direct comparisons with different preparations 
is not always possible but general trends and features of the SAXS curves persist.

\subsection{Influence of protein concentration}

As discussed above (section 3.2), the analysis of SAXS data requires the consideration of intermolecular scattering effects. A straightforward way of doing so is to record the scattering signal at identical buffer conditions but varying protein concentrations. In addition to providing an estimate for the intermolecular scattering, the variation of the protein concentration gives insight in its effect on the filament structure. Also, these experiments assist in choosing a protein concentration suitable for investigating the effects of different ions. Therefore, we compare the scattering of vimentin at selected buffer conditions and protein concentrations ranging from about $0.5 \mathrm{mg} / \mathrm{ml}$ to $5 \mathrm{mg} / \mathrm{ml}$.

\section{Wild type vimentin at different ion concentrations}

At low ionic strength, i.e. without any additional ions added to the buffer system, dominantly tetrameric vimentin is expected. Upon changing the protein concentration, only minor differences between the different curves are present when corrected for the total amount of scatterers (figure 4.2a). The most noticeable effect is the increase of the noise level at the lowest measured concentration, $0.5 \mathrm{mg} / \mathrm{ml}$. In the presence of $50 \mathrm{mM} \mathrm{KCl}$, i.e. at filament forming conditions, changes in the corrected scattering signal are quasi non-existent (figure $4.2 \mathrm{~b}$ ). The increase of noise at low protein concentrations is relatively smaller than in the absence of ions.

In the presence of $2.5 \mathrm{mM} \mathrm{MgCl}_{2}$, we also find no significant change in the scattering signal. The changes in the noise level are comparable to those at $50 \mathrm{mM} \mathrm{KCl}$. At $5.5 \mathrm{mg} / \mathrm{ml}$ the sample is slightly turbid, but the scaling behaviour of the scattering signal indicates homogeneity. When both $80 \mathrm{mM} \mathrm{KCl}$ and and $2.5 \mathrm{mM} \mathrm{MgCl} 2$ are present, there is no change in the normalized scattering signal between $0.5 \mathrm{mg} / \mathrm{ml}$ and $4 \mathrm{mg} / \mathrm{ml}$. However, in samples prepared at about $5.8 \mathrm{mg} / \mathrm{ml}$ white precipitates form in the capillary and render the sample heterogeneous. Therefore, no SAXS signal is presented, as the actual sample composition is unclear.

To summarize, for the selected salt concentrations, changes in the protein concentration between $0.5 \mathrm{mg} / \mathrm{ml}$ and $5.5 \mathrm{mg} / \mathrm{ml}$ only affect the absolute scaling of scattering linearly, as long as the sample remains homogeneous. The only sample 
(a)

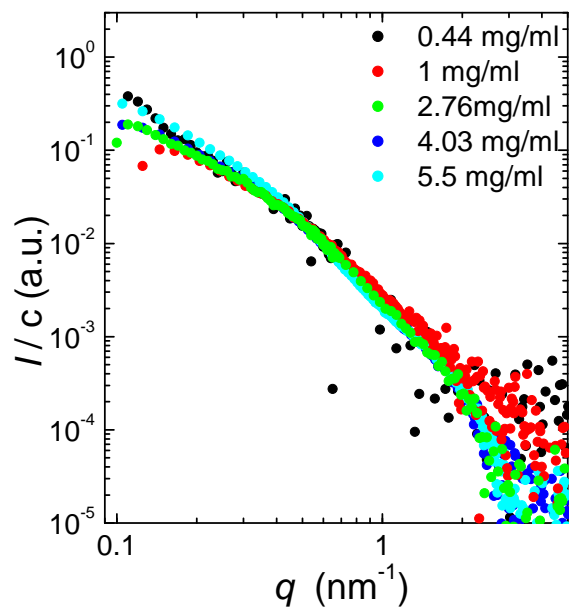

(b)

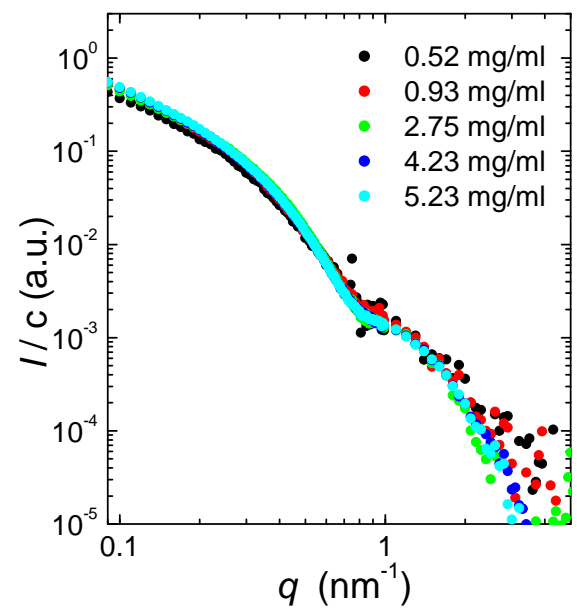

Figure 4.2: Control for protein concentration effects on the scattering signal of vimentin (wild type). All curves are normalized by the protein concentration. (a) At low ionic strength, only very small changes are observed. At $0.5 \mathrm{mg} / \mathrm{ml}$ the noise level is significantly increased. (b) At $50 \mathrm{mM} \mathrm{KCl}$, the changes in the signal are even smaller. Also, the increase in noise at low protein concentrations is less.

(a)

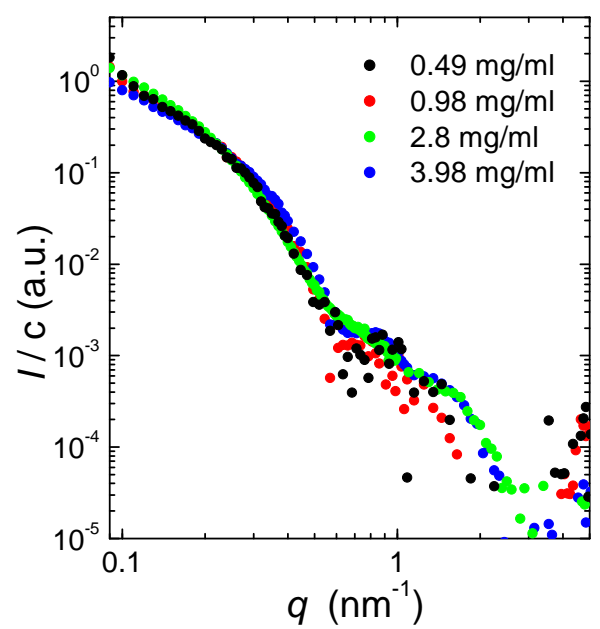

(b)

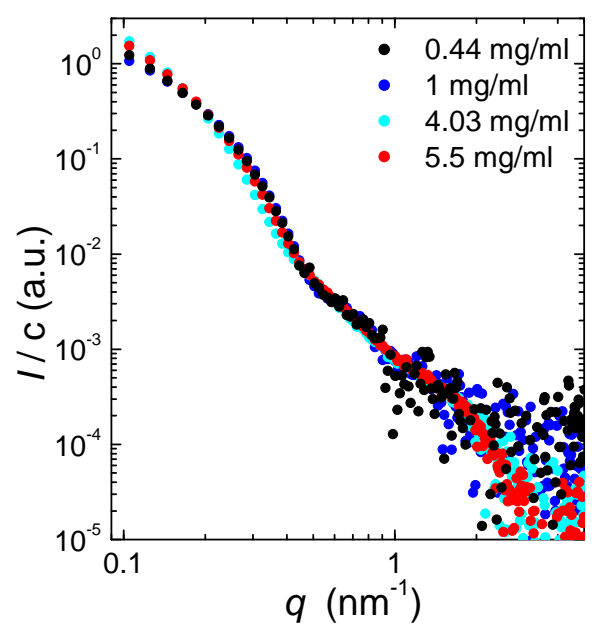

Figure 4.3: Control for protein concentration effects on the scattering signal of vimentin (wild type). All curves are normalized by the protein concentration. At both (a) $2.5 \mathrm{mM}$ $\mathrm{MgCl}_{2}$ and (b) $80 \mathrm{mM} \mathrm{KCl}, 2.5 \mathrm{mM} \mathrm{MgCl}_{2}$, there are no significant changes. At the lowest recorded concentration, the noise level is considerably increased. 
which reached an inhomogeneous state was $5.8 \mathrm{mg} / \mathrm{ml}$ protein at $2.5 \mathrm{mM} \mathrm{MgCl} 2$ in the absence of monovalent cations. As a consequence of the absence of changes in the scattering signal, all SAXS curves discussed in the following are normalized by their actual protein concentration to separate protein concentration effects from actual structural changes. Further, based on the signal to noise ratio and the protein concentrations typically reached in the protein preparation, the effect of changes in the ionic composition of the buffer is studied at about $2.75 \mathrm{mg} / \mathrm{ml}$.

\section{The mutant $Y 117 L$ at $50 \mathrm{mM} \mathrm{KCl}$}

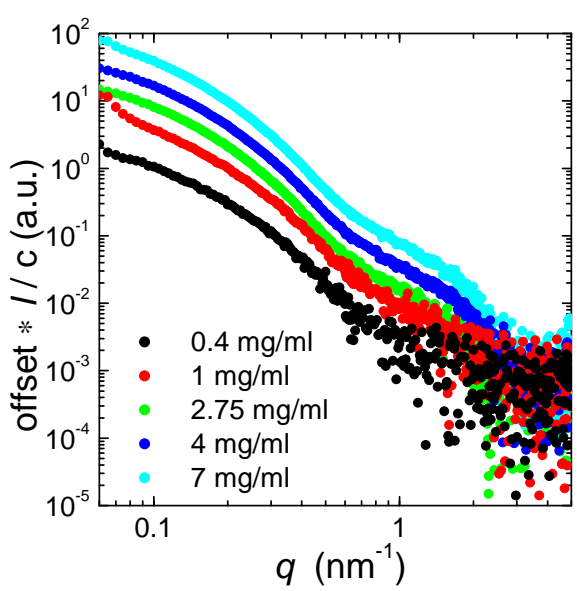

Figure 4.4: Control for protein concentration effects on the scattering signal of vimentin $(Y 117 L)$ in the presence of $50 \mathrm{mM}$ $\mathrm{KCl}$. Curves are shifted in respect to each other for ease of comparison. As the protein concentration increases, the curves become more structured.
For the stable ULF forming vimentin mutant $Y 11^{r} \mathrm{~L}$ we compare the SAXS curves for different protein concentrations at $50 \mathrm{mM} \mathrm{KCl}$, i.e. analogous to the conditions used for the wild type in figure 4.2. In contrast to the wild type, which shows no protein concentration dependence at this salt concentration, the structure of the SAXS curves of $Y 117 \mathrm{~L}$ changes with increasing protein concentration with kinks emerging at about $0.7 \mathrm{~nm}^{-1}$ and $1.7 \mathrm{~nm}^{-1}$, see figure 4.4. Additionally, the slope of the curve before about $0.1 \mathrm{~nm}^{-1}$ to $0.7 \mathrm{~nm}^{-1}$ increases with the protein concentration, as does the slope above $1.7 \mathrm{~nm}^{-1}$. At low $q$, the curve for $0.4 \mathrm{mg} / \mathrm{ml}$ slightly levels off towards $q=0$, but at higher protein concentrations the scattering continues to increase in the double-logarithmic plot. In general, the results at higher protein concentrations show some resemblance to the curves of the wild type at identical salt concentration, but those at lower protein concentration do not.

These findings imply a different protein concentration dependence of the assembly of the $Y 117 L$ mutant as opposed to the wild type, and will be further discussed in section 4.5.1 and chapter 8. All subsequent data for the $Y 117 L$ mutant are recorded at about $2.75 \mathrm{mg} / \mathrm{ml}$ for comparison with the results obtained for the vimentin wild type. 


\begin{tabular}{c|cc|cc}
{$[\mathrm{KCl}](\mathrm{mM})$} & $q_{1}(\mathrm{~nm})^{-1}$ & $R_{1}(\mathrm{~nm})$ & $q_{2}(\mathrm{~nm})^{-1}$ & $R_{2}(\mathrm{~nm})$ \\
\hline 20 & $0.85 \pm 0.02$ & 4.5 & - & - \\
50 & $0.72 \pm 0.03$ & 5.3 & - & - \\
80 & $0.69 \pm 0.02$ & 5.6 & $1.23 \pm 0.02$ & 5.7 \\
100 & $0.64 \pm 0.02$ & 6.0 & $1.13 \pm 0.02$ & 6.2
\end{tabular}

Table 4.1: Estimate of the cylinder radius based on the the location of the minima of the scattering curves as zeros of the first Bessel function of the first kind.

\subsection{Influence of salt concentration}

In the following we will address the question how the addition of different ions alters the structure of both wild type vimentin and the $Y 117 \mathrm{~L}$ mutant, as previous studies indicate different effect for mono- and divalent ions, see section. 2.1.1. Towards this goal, we describe SAXS curves for both proteins recorded at different ion concentrations and species.

\section{SAXS curves of the vimentin wild type}

Figures 4.5 and 4.6 show SAXS curves of vimentin at different salt concentrations. The Kratky plots ( $I q^{2}$ vs. $q$ ) on the right hand side ((b),(d)) emphasize changes in the mid- $q$-range and help to estimate the compactness of the sample $[69,83]$.

In figure $4.5 \mathrm{a}$ we see that the addition of potassium chloride to the protein solution leads to an increase of the scattering at low $q$-values $\left(\simeq 0.1 \mathrm{~nm}^{-1}\right)$, accompanied by a steepening of the scattering curve, resulting in less scattering at higher q. Also, whilst the scattering curve in low salt buffer levels off towards small values of $q$, and even slightly decreases, the curves at higher salt concentrations continue to rise. Further, at about $0.7 \mathrm{~nm}^{-1}$ a kink in the scattering curve, consisting of a very slight local minimum and maximum, emerges. A weaker second kink emerges at about $1.2 \mathrm{~nm}^{-1}$. All these features are more prominent at higher salt concentrations (e.g. $100 \mathrm{mM}$ ) than at lower salt concentrations (e.g. $10 \mathrm{mM}$ ) and changes are especially strong at lower salt concentrations. At higher $q$ above $2 \mathrm{~nm}^{-1}$ all scattering curves are parallel.

The local minima allow us to give a first rough estimate of filament size: Assuming perfect cylinders, the local minima correspond to the zeros of the first Bessel function of the first kind, i.e. $q R \approx 3.83$ for the first minimum and $q R \approx 7.02$ for the second one, see appendix B.3. Table 4.1 lists the position of the minima. We see that the position of the minima decreases with higher salt concentrations and that, in direct consequence, the radius increases. Further, the radii based on the second 
(a)

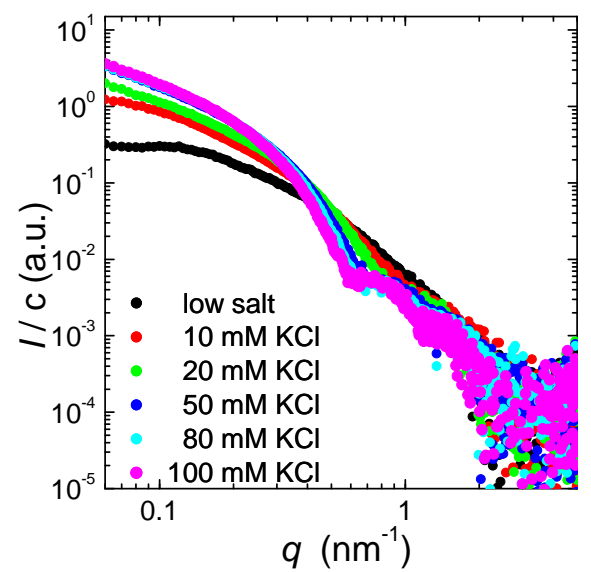

(c)

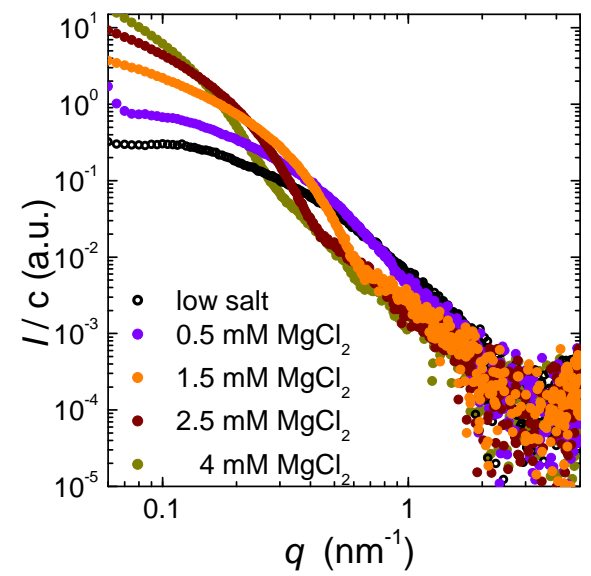

(b)

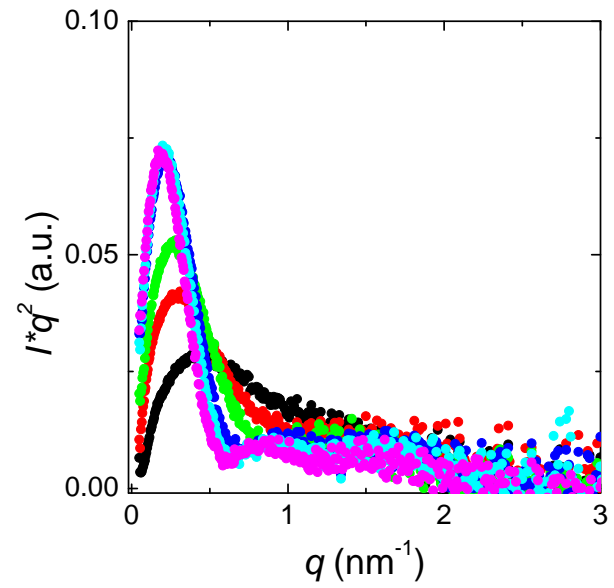

(d)

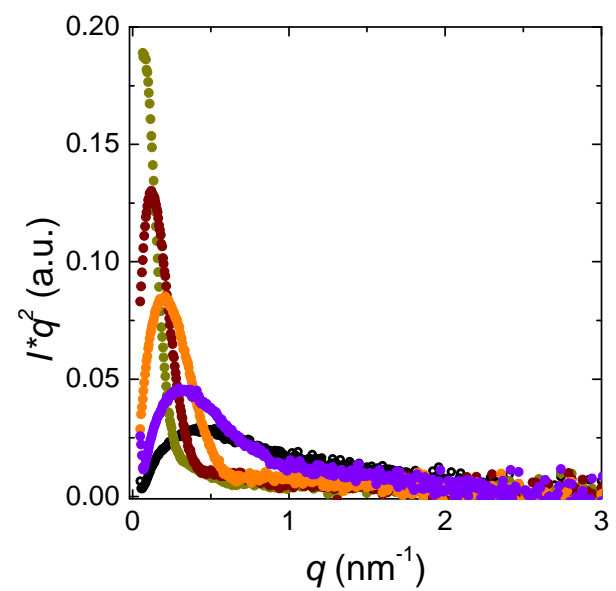

Figure 4.5: The small angle scattering signal of vimentin (wild type) recorded at different concentrations of a single cation species. All curves are recorded at about $3 \mathrm{mg} / \mathrm{ml}$ protein and normalized by the protein concentration. (a), (b) Changes in the small angle scattering of vimentin upon variation of the potassium chloride concentration. The black curve was collected at low salt buffer conditions, the red, green, blue, cyan and magenta curves at increasing concentration of monovalent potassium ions. (c), (d) Changes in the small angle scattering of vimentin upon variation of the magnesium chloride concentration. Again, the black curve was collected at low salt buffer conditions, the red and cyan curves at increasing concentration of divalent magnesium ions. 


\begin{tabular}{c|ccc}
{$\left[\mathrm{MgCl}_{2}\right](\mathrm{mM})$} & 1.5 & 2.5 & 4 \\
\hline$q(\mathrm{~nm})^{-1}$ & $0.67 \pm 0.02$ & $0.4 \pm 0.01$ & $0.31 \pm 0.03$
\end{tabular}

Table 4.2: Positions of the kinks in the double logarithmic plots of the scattering curves in the presence of only magnesium chloride.

minimum are larger than those based in the first minimum. Also, the values are larger than those observed by TEM as we will discuss in section 4.5.2.

In the Kratky plot, all curves have peaks and decay to zero at larger $q$ (figure 4.5b). The very broad and flat peak at about $0.35 \mathrm{~nm}^{-1}$ observed for tetrameric vimentin becomes considerably narrower and higher at the addition of potassium chloride and shifts to smaller $q$. At $50 \mathrm{mM} \mathrm{KCl}$, the peak position settles at $0.2 \mathrm{~nm}^{-1}$ and at lower $q$ the curves for higher salt concentrations are very similar. At $50 \mathrm{mM}$ $\mathrm{KCl}$ an additional peak, corresponding to the kink in the double-logarithmic plot, emerges at about $0.8 \mathrm{~nm}^{-1}$ which shift towards smaller $q$ with higher salt concentration. Samples prepared at $200 \mathrm{mM} \mathrm{KCl}$ quickly became heterogeneous, therefore no SAXS signal was recorded.

When adding magnesium chloride instead of potassium chloride to the vimentin, the changes observed at low salt concentrations appear to be similar (figure 4.5c): An increase of scattering at small $q$ and an overall steeper scattering curve. At higher salt concentrations the slope at small $q$ continues to become steeper and a kink in the scattering curve emerges at about $0.6 \mathrm{~nm}^{-1}$. In contrast to the kinks found in presence of potassium chloride, this kink does not suggest local maxima and minima and after the kink all curves assume a similar constant slope of about -2.8 (determined by a power law fit for $2.5 \mathrm{mM} \mathrm{MgCl}_{2}$ between $0.5 \mathrm{~nm}^{-1}$ and $3 \mathrm{~nm}^{-1}$ ). Table 4.2 gives the position of the kinks for different magnesium concentrations. The shift towards smaller $q$ with increasing salt concentration is considerably stronger than the shift in the position of the minima observed in the presence of potassium chloride. At $4 \mathrm{mM} \mathrm{MgCl}_{2}$ the position of the kink is hard to discern as the slope before and after it are nearly identical. We will later see (sections 4.4 .2 and 4.5.2) that the kink originates from the superposition of the scattering from different regions of the cross-section.

In the Kratky plot the peak continuously sharpens and moves to smaller $q$ as the salt concentration increases. In contrast to the data for potassium chloride no secondary peak emerges and no saturation can be observed. Samples prepared at $5 \mathrm{mM} \mathrm{MgCl} 2$ turned turbid and samples at even higher concentrations became visibly heterogeneous. Therefore no SAXS data for such samples are shown. 
(a)

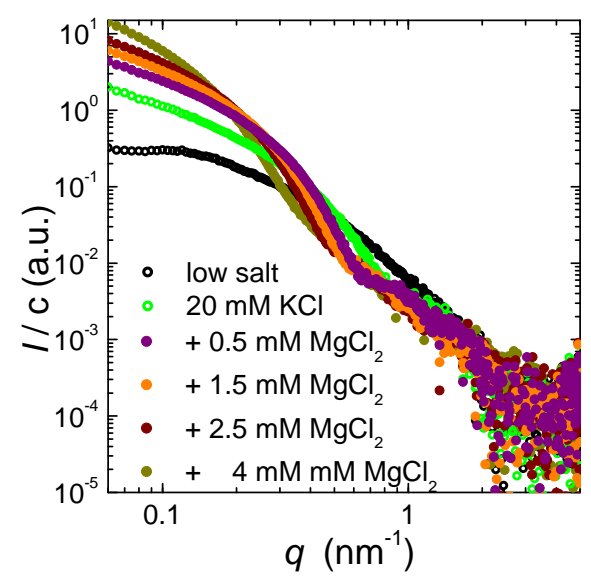

(c)

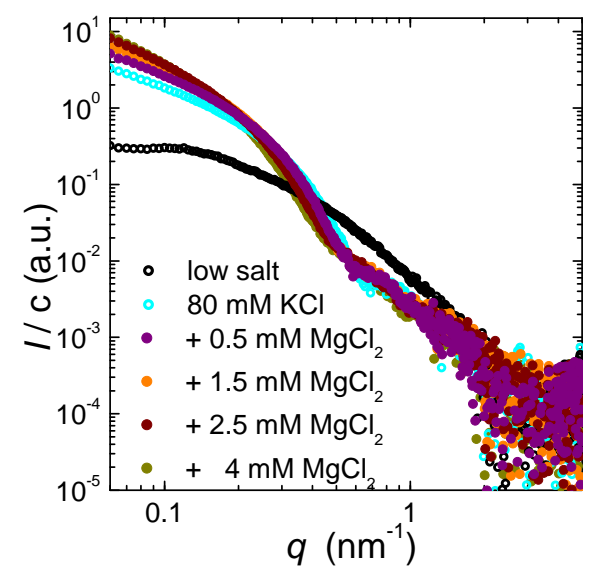

(b)

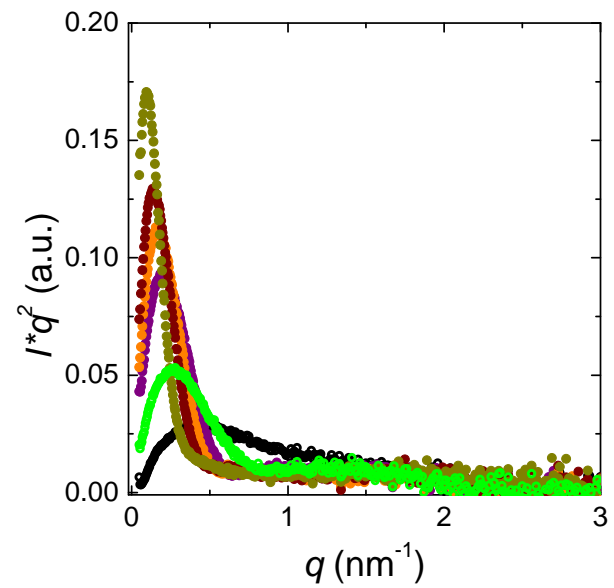

(d)

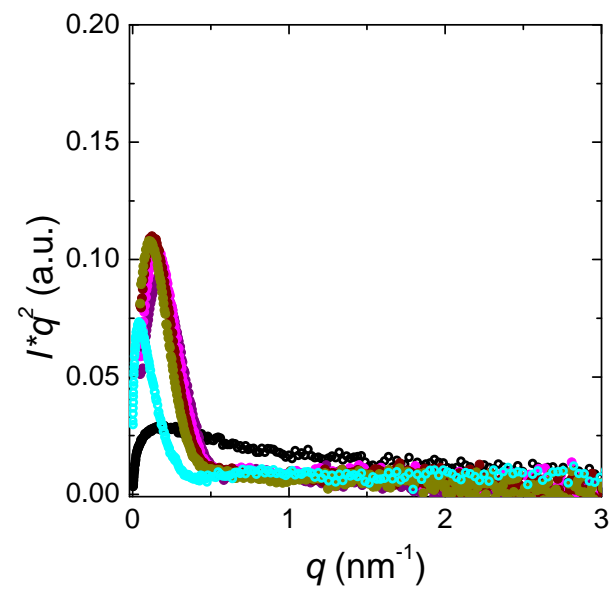

Figure 4.6: Changes in the small angle scattering of vimentin upon changing the magnesium chloride concentration at a fixed potassium chloride concentration. The black curves were collected at low salt buffer conditions, the green and cyan curves in the absence of magnesium chloride. (a), (b) At $20 \mathrm{mM} \mathrm{KCl}$ we see distinct changes in the scattering curves as magnesium chloride concentration increases. (c),(d) At $80 \mathrm{mM} \mathrm{KCl,} \mathrm{changes} \mathrm{are}$ less obvious.

Finally, we investigate how the addition of magnesium chloride changes the scattering signal at $20 \mathrm{mM}$ and $80 \mathrm{mM} \mathrm{KCl} \mathrm{(fig.4.6).} \mathrm{At} 20 \mathrm{mM} \mathrm{KCl}, 0.5 \mathrm{mM} \mathrm{MgCl}_{2}$ induces a kink that resembles those found in the presence of only potassium chloride. Its location $\left(0.68 \pm 0.01 \mathrm{~nm}^{-1}\right)$ corresponds to the minimum of the form factor of a perfect cylinder with a radius of $5.6 \mathrm{~nm}$. For $1.5 \mathrm{mM} \mathrm{MgCl}_{2}$ the kink is slightly more 
pronounced and moves to $0.58 \pm 0.01 \mathrm{~nm}^{-1}$, corresponding to a radius of $6.6 \mathrm{~nm}$. At $2.5 \mathrm{mM}$ the kink position reduces to $0.5 \mathrm{~nm}^{-1}$ and its shape more closely resembles those found in the presence of only magnesium chloride. At $4 \mathrm{mM} \mathrm{MgCl}_{2}$ one can at most guess a local maximum at $0.4 \mathrm{~nm}^{-1}$. Due to the different shapes of the kinks, it is likely that they do not stem from the same type of structural features and direct comparison of their locations might lead to mis-interpretations. In the Kratky plot the height of the peak exceeds the highest peak observed in only potassium chloride already at additional $0.5 \mathrm{mM} \mathrm{MgCl}_{2}$. It continues to grow and move to smaller $q$ as the magnesium concentration increases but does not reach the height of the peak reached in the absence of potassium chloride. Samples with $5 \mathrm{mM} \mathrm{MgCl}_{2}$ became heterogeneous.

At $80 \mathrm{mM} \mathrm{KCl}$, there are significantly less changes visible in the double logarithmic plot of the scattering curve than at $20 \mathrm{mM}$. Upon the addition of $0.5 \mathrm{mM} \mathrm{MgCl} 2$ the second local minimum (at $1.2 \mathrm{~nm}^{-1}$ ) vanishes and the first one moves to slightly smaller $q$. Simultaneously, the scattering at low $q$ increases. These trends continue for $1.5 \mathrm{mM} \mathrm{MgCl}_{2}$, but the shape of the kink comes to resemble the shape observed in the absence of potassium chloride. At $2.5 \mathrm{mM}$ and $4 \mathrm{mM} \mathrm{MgCl}_{2}$ the shape of the kink and the subsequent slope of the curve closely resembles those observed in the absence of potassium chloride. In the Kratky plot, the most striking feature is the shift of the peak to larger $q$ upon addition of magnesium chloride. Further increase of the magnesium concentration returns the peak to smaller $q$, but it does not reach its initial value. Overall the change in peak shape and position after the initial shift is small and the peak does not reach the same height as for $20 \mathrm{mM} \mathrm{KCl}$. Simultaneously to the changes in the main peak, the second peak at $0.8 \mathrm{~nm}^{-1}$ decays with increasing magnesium. At $80 \mathrm{mM} \mathrm{KCl}$ samples with $5 \mathrm{mM} \mathrm{MgCl} 2$ remained homogeneous, but at $8 \mathrm{mM} \mathrm{MgCl}_{2}$, samples were heterogeneous.

\section{SAXS curves of the $Y 117 L$ mutant}

For the $Y 11^{\prime} 7 \mathrm{~L}$ mutant, as for the wild type, we study the effect of potassium chloride on protein structure by comparing the scattering curves. Initial changes at $10 \mathrm{mM}$ potassium chloride are very similar to those observed in the wild type: A strong increase in scattering at low $q$ and a steeper slope of the overall curve. At $20 \mathrm{mM}$ $\mathrm{KCl}$, these changes continue but additionally a slight kink emerges, which becomes more prominent as the salt concentration continues to increase, see figure 4.7. In 
(a)

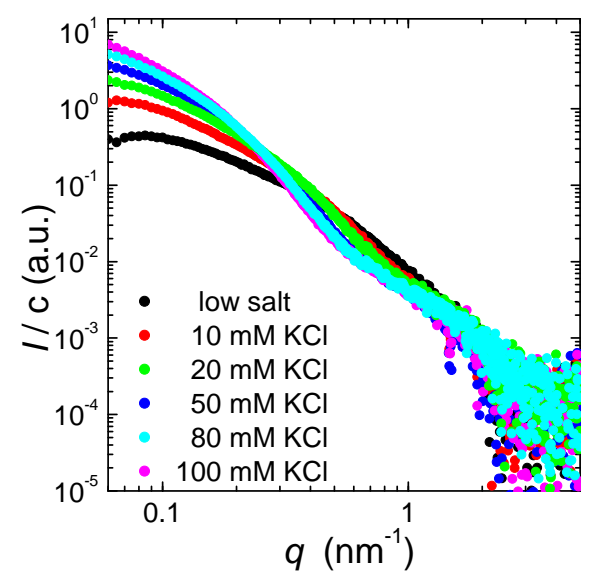

(b)

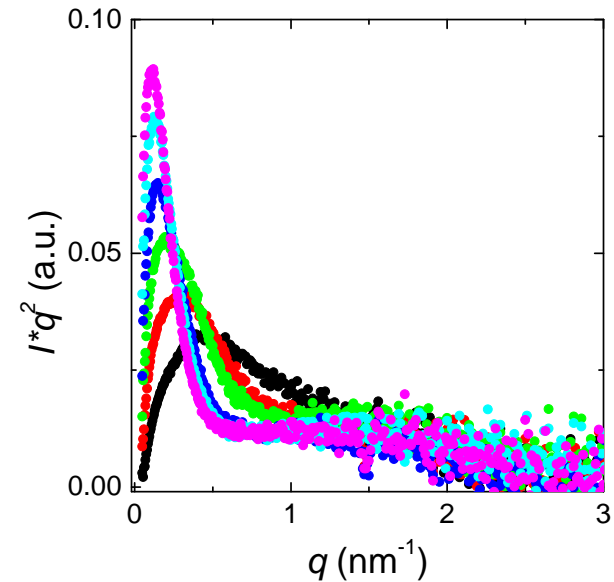

Figure 4.7: Changes in the small angle scattering of the vimentin mutant $Y 117 \mathrm{~L}$ upon changing the potassium chloride concentration in the absence of magnesium chloride. The black curves were collected at low salt buffer conditions, the red, green, blue, cyan and magenta curves represent increasing potassium chloride concentration.

\begin{tabular}{c|cccc}
{$[\mathrm{KCl}](\mathrm{mM})$} & 20 & 50 & 80 & 100 \\
\hline$q\left(\mathrm{~nm}^{-1}\right)$ & $0.85 \pm 0.01$ & $0.55 \pm 0.05$ & $0.53 \pm 0.05$ & $0.47 \pm 0.05$
\end{tabular}

Table 4.3: Positions of the kinks in the double logarithmic plots of the scattering curves of $Y 117 \mathrm{~L}$ in the presence of potassium chloride.

contrast to the wild type in the presence of potassium chloride, this kink does not represent a local minimum/maximum pair but instead a change in the curvature. Table 4.3 summarizes the location of these turning points. The kinks observed are less sharp than those observed for the wild type in the presence of magnesium chloride, but resemble more the turning point of an S-like shape. In the Kratky plot the movement of the peak to smaller $q$ values and its sharpening resemble the changes observed in wild type vimentin. However, the height of the peak does not saturate but continues to increase.

In the presence of $2.5 \mathrm{mM} \mathrm{MgCl}_{2}$ the scattering curves of $Y 117 \mathrm{~L}$ are very similar in the absence of potassium chloride and at different potassium chloride concentrations (figure 4.8). All three curves display an S-shape similar to those observed at higher potassium chloride concentrations. In the absence of potassium chloride, this S-shape is the most pronounced and at $80 \mathrm{mM} \mathrm{KCl}$ it is the least pronounced.

In the Kratky plot, all curves are similarly shaped and the peak position only 
(a)

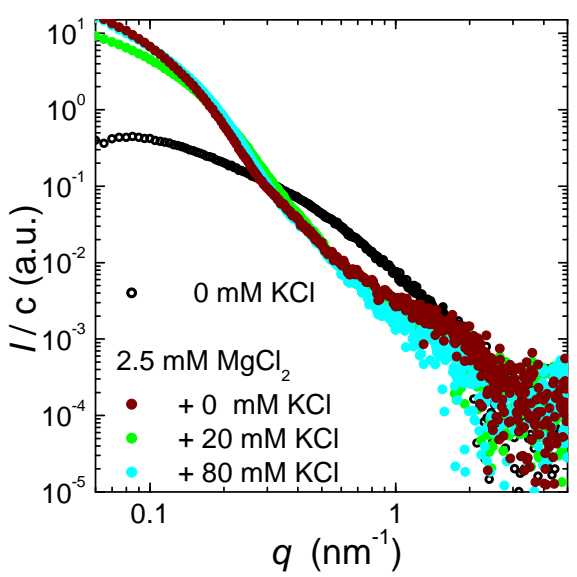

(b)

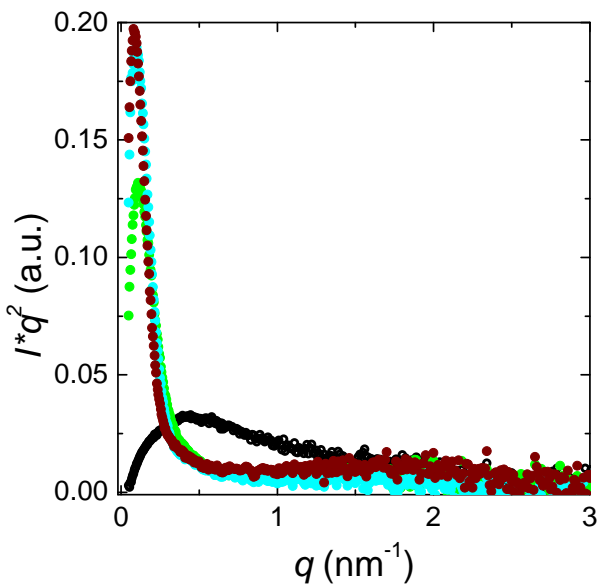

Figure 4.8: Changes in the small angle scattering of the vimentin mutant $Y 117 \mathrm{~L}$ upon changing the potassium chloride concentration in the presence of $2.5 \mathrm{mM} \mathrm{MgCl}_{2}$. The black curves were collected at low salt buffer conditions, the brown curve at $2.5 \mathrm{mM}$ $\mathrm{MgCl}_{2}$ without potassium chloride, the green curve at additional $20 \mathrm{mM} \mathrm{KCl}$ and the cyan curve at $80 \mathrm{mM} \mathrm{KCl}$.

moves slightly. The differences in the absence of potassium chloride and at $80 \mathrm{mM}$ $\mathrm{KCl}$ are very small, but at $20 \mathrm{mM} \mathrm{KCl}$ the peak is considerably smaller.

When directly comparing the scattering curves of the vimentin wild type with those of the $Y 117 \mathrm{~L}$ mutant at identical salt concentration in figure 4.9, we see that for both low salt buffer and $10 \mathrm{mM} \mathrm{KCl}$ the curves are practically identical. This implies that the form of the tetramer of the mutant is identical to that of the wild type and also that structures formed at low monovalent ion concentrations are identical. In contrast to this observation the scattering curves at $100 \mathrm{mM} \mathrm{KCl}$ and $2.5 \mathrm{mM} \mathrm{MgCl}_{2}$ differ strongly in the general shape of the curve: At $100 \mathrm{mM} \mathrm{KCl}$ the local minima and maxima which can be identified for the wild type are completely missing for the mutant as is the sharp change in curvature observed at $2.5 \mathrm{mM} \mathrm{MgCl}_{2}$. This implies that at these salt concentrations the structures formed by the wild type and the mutant do not match. 
(a)

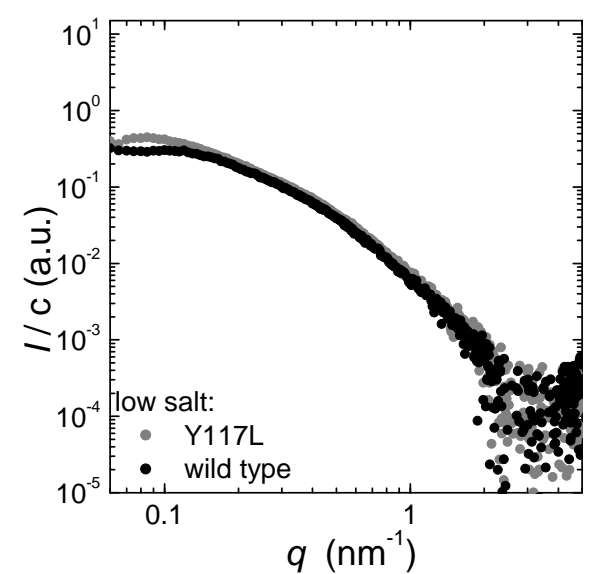

(c)

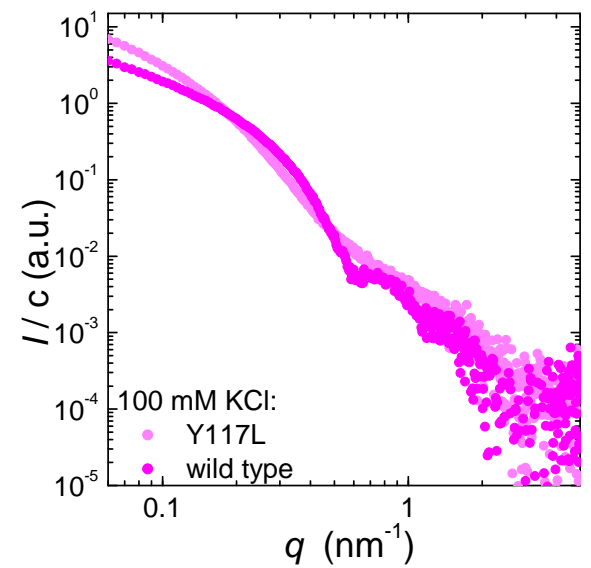

(b)

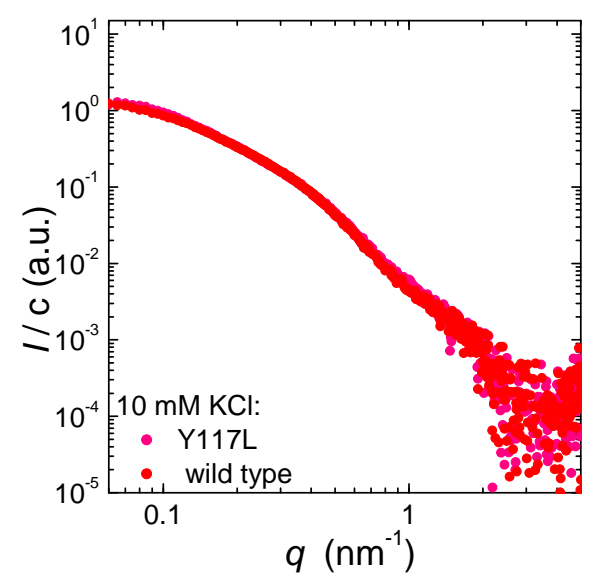

(d)

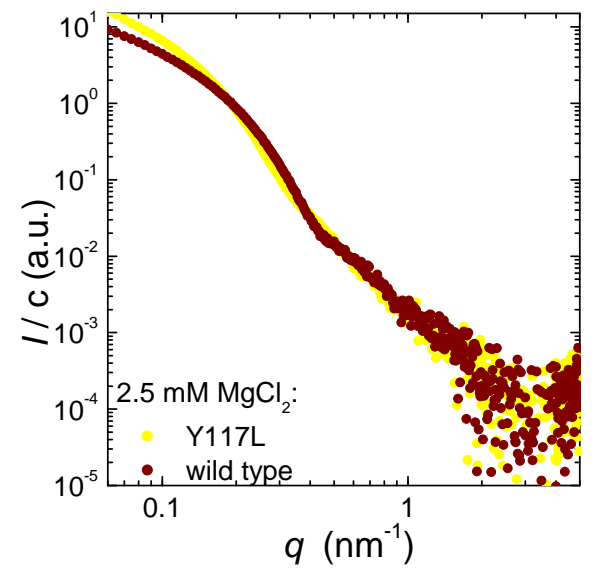

Figure 4.9: Exemplary comparisons between the scattering curves of wild type vimentin and the $Y 117 \mathrm{~L}$ mutant at (a) low salt, (b), $10 \mathrm{mM} \mathrm{KCl,} \mathrm{(c)} 100 \mathrm{mM} \mathrm{KCl}$ and (d) $2.5 \mathrm{mM}$ $\mathrm{MgCl}_{2}$. The darker color always represents the wild type, the lighter color the mutant. At low salt and $10 \mathrm{mM} \mathrm{KCl}$, the curves are nearly identical but at $100 \mathrm{mM} \mathrm{KCl}$ and at $2.5 \mathrm{mM} \mathrm{MgCl} 2$ the wild type curves possess significantly sharper features. 


\subsection{Quantitative analysis}

From the SAXS curves at different ionic compositions it is obvious that the structure of vimentin filaments is strongly influenced by ions. In the following, we apply both model-free and model-based approaches to quantify those changes and better understand the structure of the filaments.

\section{Effect of finite filament length on the SAXS curves}

Before interpreting the SAXS curves we need to estimate whether the observed changes in them are due only to changes in the cross-section or possibly also due to changes in the filament length.

The curves considered for the different ionic environments reach a minimal $q_{\min }$ of $0.05 \mathrm{~nm}^{-1}$. Therefore we need to consider what the largest structural range which affects the structure of the curves is. A first estimate is given by $d=2 \pi / q_{\min }=$ $125 \mathrm{~nm}$, which roughly corresponds to the length of two vimentin tetramers or ULFs. But how much does the form of the scattering curve actually change with the length of (short) filaments?

To answer this question we compare the theoretical scattering curves of perfect cylinders with a radius of $5 \mathrm{~nm}$ and lengths between $30 \mathrm{~nm}$ and $105 \mathrm{~nm}$. When we use no further approximations (lines in figure 4.10), we can observe several effects of increasing length: The local minima of the curve become deeper, the curve before the first minimum becomes smoother and at very low $q$ the curves turn upward instead of leveling off towards $q=0$. Of all these features, the change in slope is the most resistant against polydispersity as longitudinal polydispersity smooths the curve before the first minimum and radial polydispersity reduces the depth of the minima. Therefore, the slope towards low $q$ is the most reliable indicator of length. Between $30 \mathrm{~nm}$ and $75 \mathrm{~nm}$ we see characteristic changes in slope, but between $75 \mathrm{~nm}$ and $105 \mathrm{~nm}$ the changes are only minor. Therefore, $75 \mathrm{~nm}$ pose a realistic upper limit for possible estimates of the cylinder length, especially for cylinders with unknown cross-section which also affect the shape of the curve and hinder length determination. 


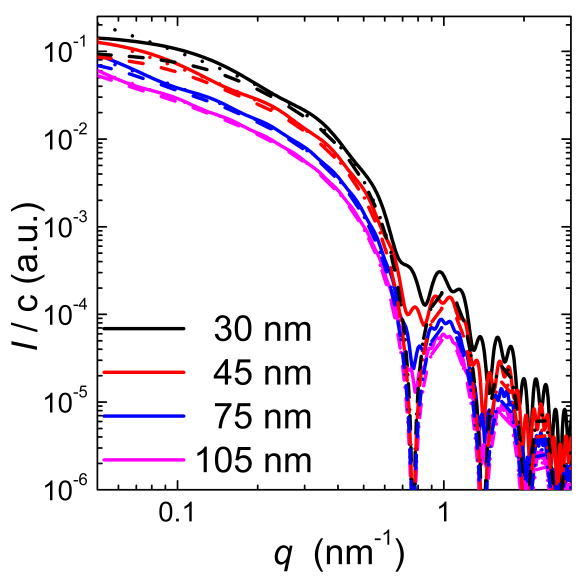

Figure 4.10: Comparison of the theoretical scattering curves for perfect cylinders with a radius of $5 \mathrm{~nm}$ and different length between $30 \mathrm{~nm}$ and $105 \mathrm{~nm}$. The solid lines correspond to the form factor calulated directly from eq. 3.6, the dashed lines correspond to the long rod approximation (eq. 3.7) with the longitudinal Neugebauer form factor eq. 3.8 and the dotted lines approximate the Neugebauer form factor with $\pi / q L$

longest investigated case of $105 \mathrm{~nm}$ both approximations describe the curve, except for the depth of the minima, very well. Interestingly for cylinders of $30 \mathrm{~nm}$ and $45 \mathrm{~nm}$ length, the very long filament approximation describes the curves better than the Neugebauer approximation. We also see that for cylinders longer than $45 \mathrm{~nm}$ the very long filament approximation describes the curves above $0.1 \mathrm{~nm}^{-1}$ outside the minima well. For even longer filaments the very long filament approximation for interpretation of the data is certainly justified, as we see that the description of the minima improves with cylinder length. Consequently, in the presence of potassium or magnesium chloride we can safely assume that the scattering, except for the total scaling, only depends the structure of the cross-section on the complete available $q$ range. In low salt buffer, this holds above about $q=0.15 \mathrm{~nm}^{-1}$, as $L q>10$ holds for larger $q$.
Another question which needs to be addressed in this context is how large the error is when we separate the longitudinal and cross-sectional part of the form factor as discussed in section 3.2. The dashed and dotted lines in figure 4.10 correspond to the Neugebauer approximation (eq. 3.8) and its simplification for very long filaments (eq. 3.10) respectively. The most obvious effect of these approximations is that the local minima of the scattering curve now go down to 0 . For $30 \mathrm{~nm}$ long cylinders both approximations fail to describe the scatproximation underestimates the scattering and the very long filament approximation corresponds to an increase of scattering towards $q=0$ which is not the case for short cylinders. For the tering at low $q$ : The Neugebauer ap- 


\subsubsection{Model-free analysis}

Model-free approaches to the interpretation of SAXS curves allow us to obtain general structural information without the bias of a model. Their results can help in building actual models and tuning their parameters. Despite being model-free, they still rely on some assumptions. For the subsequent analysis, we assume that for both tetrameric and filamentous vimentin the scattering in a selected $q$-range is governed by the cross-section.

\section{Guinier approximation}

The Guinier approximation for long filaments gives a first estimate of the radial size of the filaments via the cross-sectional radius of gyration and the forward scattering intensity including their changes, using only the small-q region, see section 3.4.1. Figure 4.11 shows some exemplary fits for different samples and the $q$-regions considered for the fit (filled circles) in a Guinier plot $\left(\log (I q)\right.$ over $\left.q^{2}\right)$, where the slope of the curve at small $q$ is proportional to the radius of gyration. For easier comparison of the slopes all curves are scaled for the forward scattering.

For the wild type of vimentin, we see that all curves have a linear region in the plot and that increases in the salt concentration increase the slope in the linear region, i.e. the cross-sectional radius of gyration becomes larger. Consequently, the Guinier regime, defined by $\left(q R_{C}\right)^{2}<2$ becomes smaller with only 12 data points contributing at $4 \mathrm{mM} \mathrm{MgCl}_{2}$. We see that the changes in the slope between different potassium chloride concentrations in the absence of magnesium chloride are small in comparison to those between different magnesium chloride concentrations in the absence of potassium chloride. As the potassium chloride concentration increases, the slopes move closer together.

Figure 4.12 shows a comparison of the squared cross-sectional radii of gyration and forward scattering amplitudes for different salt concentrations. We see that in the abscence of magnesium chloride, the radius of gyration of the filaments and the forward scattering amplitude rise together and saturate for higher concentrations with $R_{c}^{2}$ in the range of $25 \mathrm{~nm}^{2}$. In contrast, in the presence of only magnesium chloride, both the radius of gyration of the filaments and the forward scattering amplitude continue to increase with increasing ion concentration. $R_{c}^{2}$ reaches about $25 \mathrm{~nm}^{2}$ already at $1.5 \mathrm{mM} \mathrm{MgCl}_{2}$ and at $4 \mathrm{mM} \mathrm{MgCl}_{2}$ it is about $175 \mathrm{~nm}^{2}$. In the presence of $20 \mathrm{mM} \mathrm{KCl}$ and $80 \mathrm{mM} \mathrm{KCl}$ the increase in $R_{c}^{2}$ is nearly linear, with a higher slope for $20 \mathrm{mM} \mathrm{KCl}$. At $80 \mathrm{mM} \mathrm{KCl}, R_{c}^{2}$ increases faster than the forward 
(a) $0 \mathrm{mM} \mathrm{MgCl} 2$

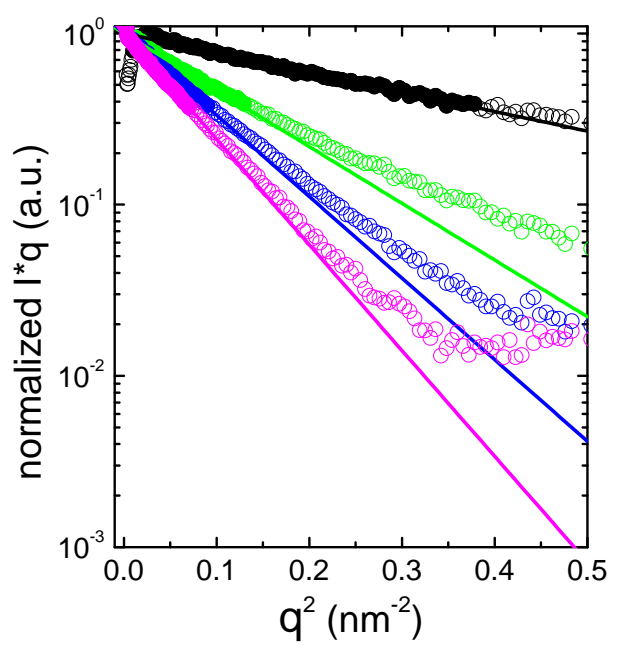

(c) $20 \mathrm{mM} \mathrm{KCl}$

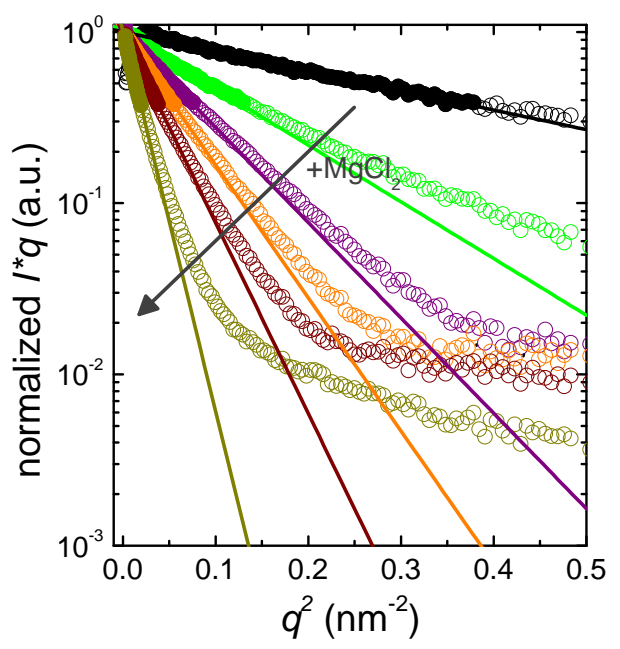

(b) $0 \mathrm{mM} \mathrm{KCl}$

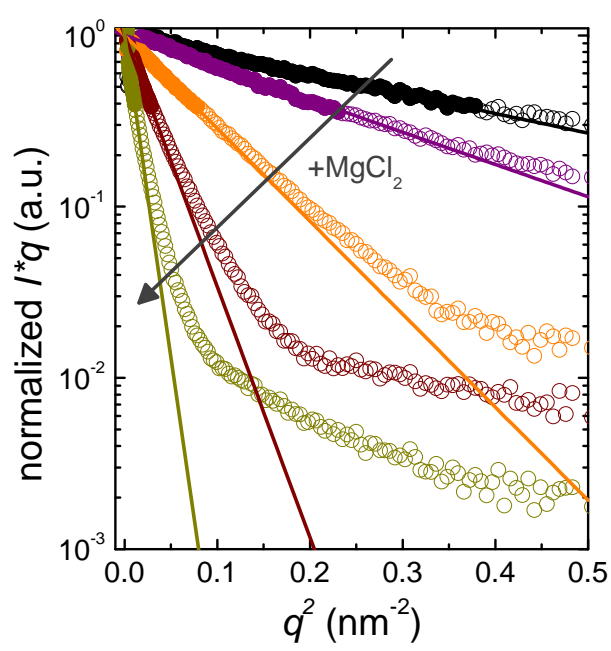

(d) $80 \mathrm{mM} \mathrm{KCl}$

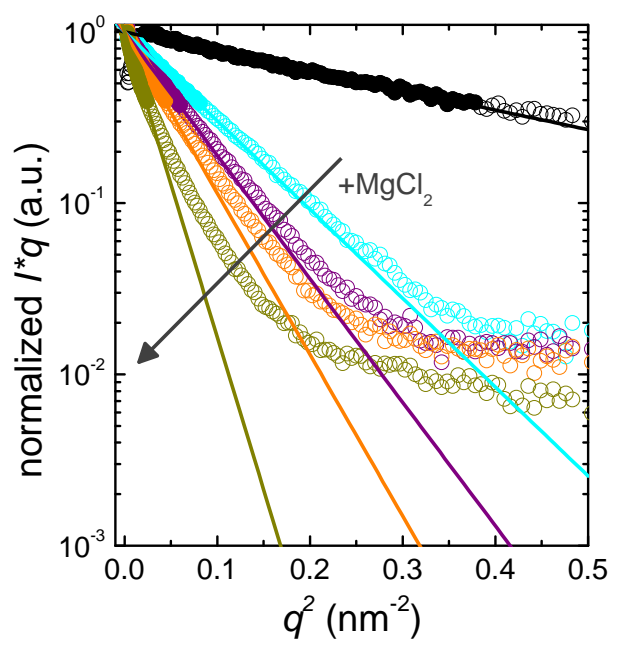

Figure 4.11: Exemplary radial Guinier fits of the vimentin wild type for different salt concentrations, scaled by the forward scattering amplitude for (a) increasing potassium chloride concentration, (b) increasing magnesium chloride concentration in the absence of potassium, (c) increasing magnesium chloride concentration at $20 \mathrm{mM} \mathrm{KCl}$ and (d) increasing magnesium chloride concentration at $80 \mathrm{mM} \mathrm{KCl}$. The solid circles mark the $q$-range used for the respective fit, the open circles the curve outside the fitting region and the lines the fits. The color code is identical to the figures 4.5 and 4.6: the black curve represents low salt conditions, green, blue, cyan and magenta increasing potassium chloride concentration and violet, orange, brown and yellow increasing magnesium chloride concentrations. 
(a) $0 \mathrm{mM} \mathrm{MgCl} 2$

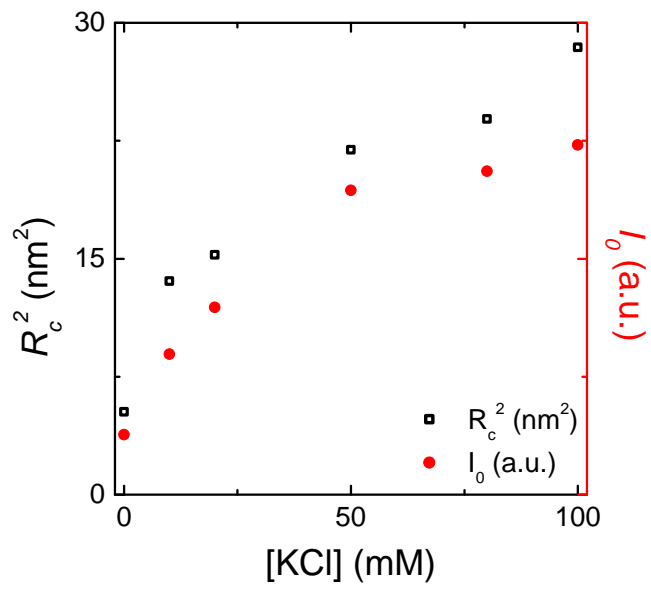

(c) $20 \mathrm{mM} \mathrm{KCl}$

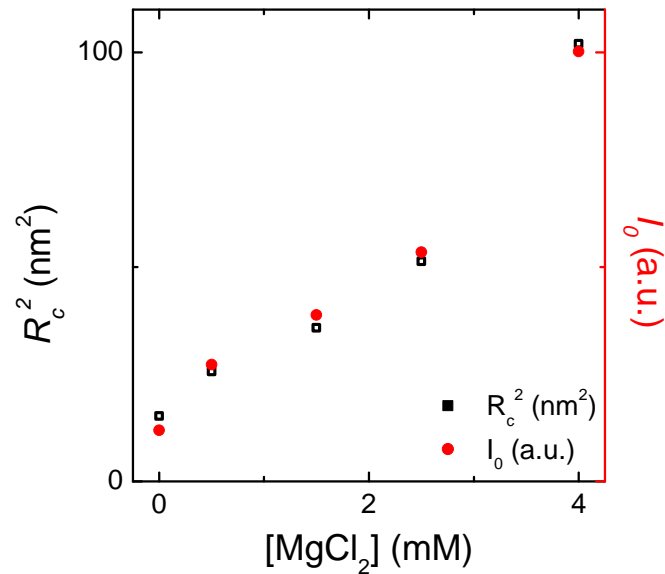

(b) $0 \mathrm{mM} \mathrm{KCl}$

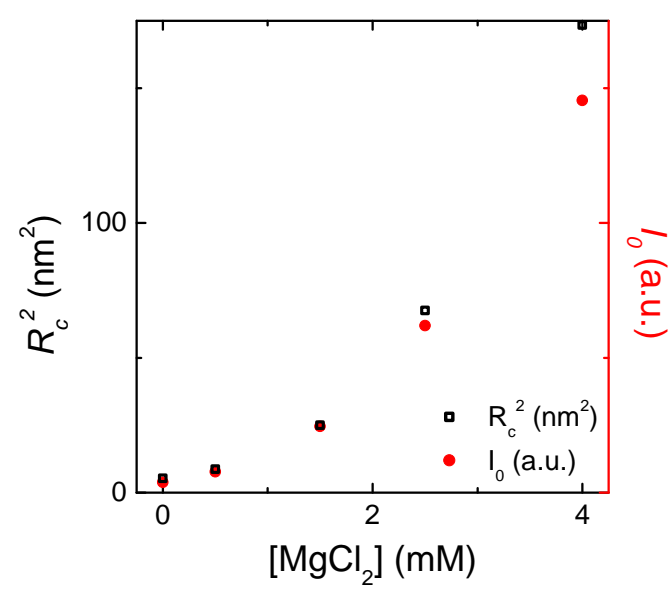

(d) $80 \mathrm{mM} \mathrm{KCl}$

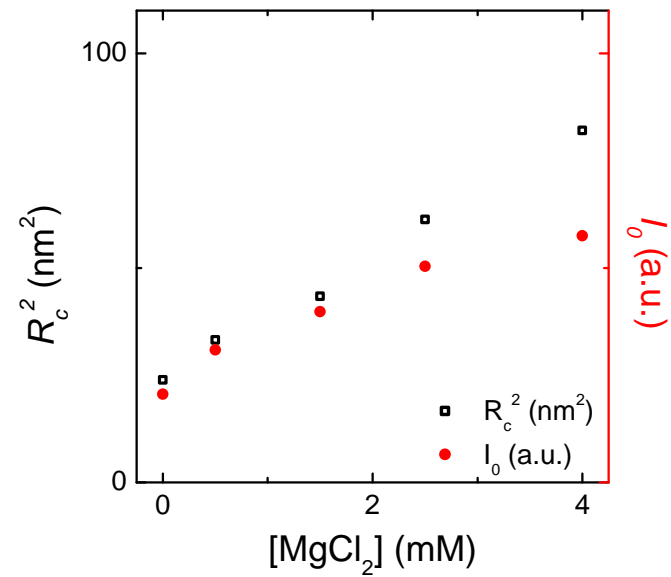

Figure 4.12: Cross-sectional gyration radii and forward scattering amplitude of the vimentin wild type obtained by Guinier fits for different salt concentrations. (a) Different potassium chloride concentrations in the abscence of magnesium chloride. (b) Different magnesium chloride concentrations in the abscence of potassium chloride. (c) Different magnesium chloride concentrations at $20 \mathrm{mM} \mathrm{KCl}$. (d) Different magnesium chloride concentrations at $80 \mathrm{mM} \mathrm{KCl}$. 
scattering whereas for all other investigated cases their ratio only changes slightly.

For the $Y 117 L$ mutant, in the abscence of magnesium chloride the $q$-range contributing to the Guinier regime is rather limited and the curves are steeper than for the wild type (figure 4.13a). Both the square of the radius of gyration and the forward scattering amplitude increase linearly with the potassium chloride concentration, but the slope of the radius of gyration is steeper (figure 4.13c). At $100 \mathrm{mM} \mathrm{KCl}$ $R_{c}^{2}$ reaches about $80 \mathrm{~nm}^{2}$, which is more than three times higher than for the wild type. The addition of $2.5 \mathrm{mM} \mathrm{MgCl}_{2}$ further increases the slopes of the curves in the Guinier plot and their differences become smaller (figure 4.13b). The ratio of the forward scattering amplitude to the radius of gyration squared remains roughly constant. The radius of gyration is smallest at $20 \mathrm{mM} \mathrm{KCl}$ with $R_{c}^{2} \approx 75 \mathrm{~nm}^{2}$ and highest in the absence of potassium chloride with $R_{c}^{2} \approx 150 \mathrm{~nm}^{2}$. All three values are larger than for the wild type with the largest difference for $0 \mathrm{mM} \mathrm{KCl}$.

\section{Pair distance distribution functions}

The pair distance distribution function of a molecule is related to the auto-correlation of its electron density distribution and provides model-free structural information based on the complete $q$-range. However, its shape is sensitive to parameters chosen for the transformation such as the maximal expected distance in the macromolecule. It can be calculated from the SAXS curve by indirect Fourier transformation when the resolution at small $q$ is sufficient, see section 3.4.1. For a perfect cylinder, the cross-sectional PDDF is a bell shaped curve with its maximum at the cylinder radius. For a hollow cylinder, the maximum moves to larger distances and excentrity or polydispersity result in extended tails. The presence of more than one peak in the PDDF indicates several distinct sub-species.

Figure 4.14 shows the normalized cross-sectional PDDFs of the vimentin wild type at different salt concentrations. In the absence of any additional ions, the PDDF has one narrow peak at $1.5 \mathrm{~nm}$ with one additional small shoulder at about $4 \mathrm{~nm}$. The addition of $10 \mathrm{mM} \mathrm{KCl}$ moves the main peak to $3.5 \mathrm{~nm}$ and reduces the peak at $1.5 \mathrm{~nm}$ to a shoulder. With increasing potassium chloride concentration, the shoulder at smaller $r$ diminishes and eventually vanishes and the second peak moves to larger $5.5 \mathrm{~nm}$ for $100 \mathrm{mM}$. Additionally, the peaks become more symmetric. Most curves display a long tail towards large distances which at least partially (especially in the case of $10 \mathrm{mM} \mathrm{KCl}$ ) might be an artifact from the calculation. 
(a) $0 \mathrm{mM} \mathrm{MgCl} 2$

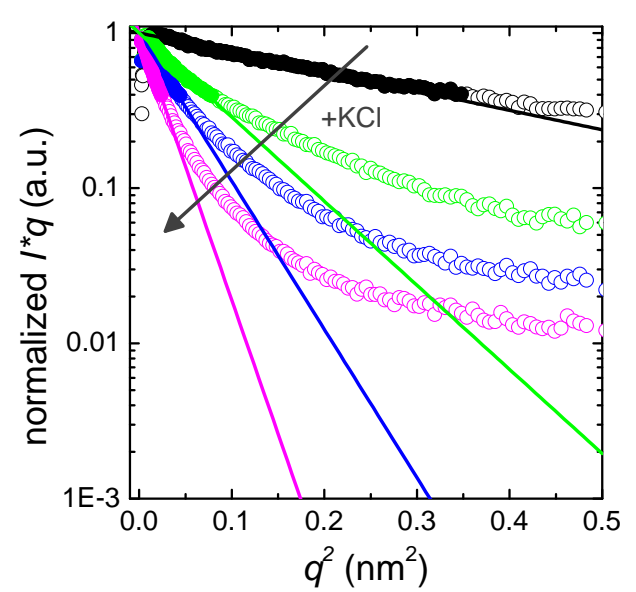

(c) $0 \mathrm{mM} \mathrm{MgCl} 2$

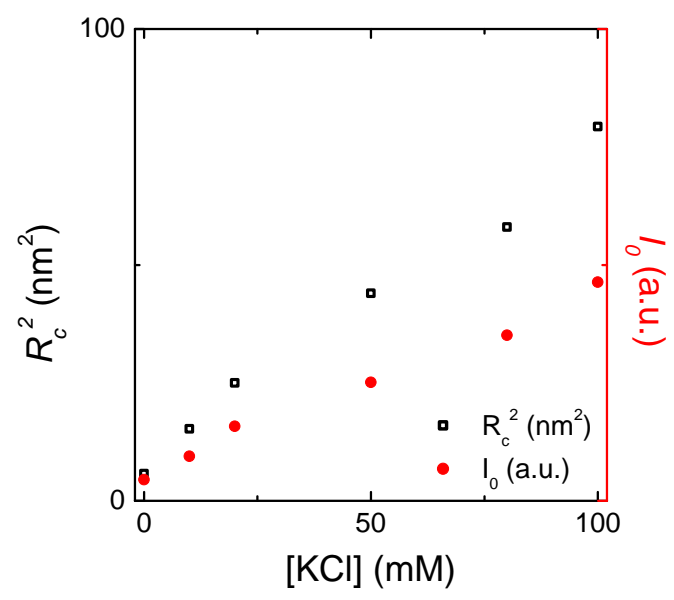

(b) $2.5 \mathrm{mM} \mathrm{MgCl} 2$

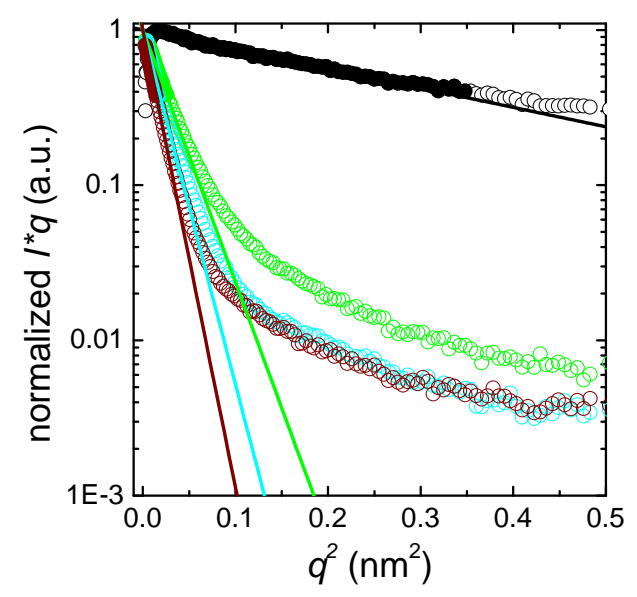

(d) $2.5 \mathrm{mM} \mathrm{MgCl} 2$

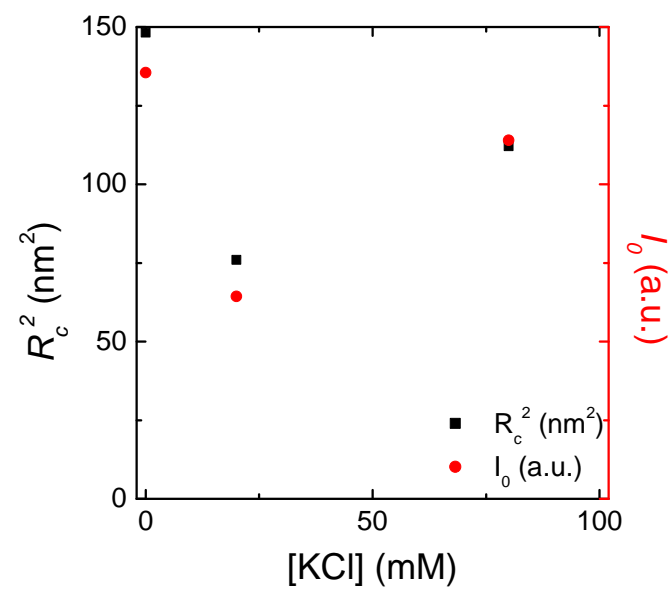

Figure 4.13: (a), (b) Exemplary cross-sectional Guinier fits of the $Y 117 \mathrm{~L}$ mutant for different salt concentrations, scaled by the forward scattering amplitude for cross-sectional gyration radii and forward scattering amplitude for increasing potassium chloride concentration at $0 \mathrm{mM} \mathrm{MgCl}$ and $2.5 \mathrm{mM} \mathrm{MgCl}_{2}$, respectively. The color code corresponds to figures 4.7 and 4.8 with the black curve representing low salt concentrations, brown representing $2.5 \mathrm{mM} \mathrm{MgCl} 2$ only, and green, blue cyan and magenta representing increasing potassium chloride concentrations. (c), (d) Cross-sectional radii of gyration and forward scattering amplitudes obtained from the Guinier fits at $0 \mathrm{mM} \mathrm{MgCl} 2$ and $2.5 \mathrm{mM} \mathrm{MgCl}_{2}$, respectively. 
The addition of $0.5 \mathrm{mM} \mathrm{MgCl}$ in the absence of potassium chloride shifts the peak

(a) $0 \mathrm{mM} \mathrm{MgCl} 2$

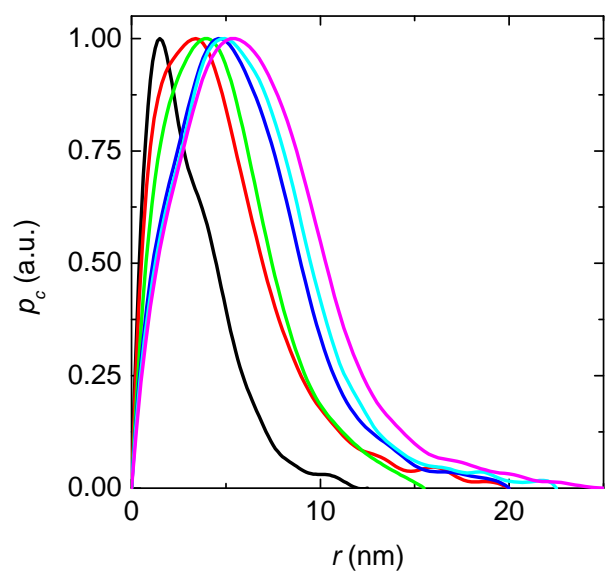

(c) $20 \mathrm{mM} \mathrm{KCl}$

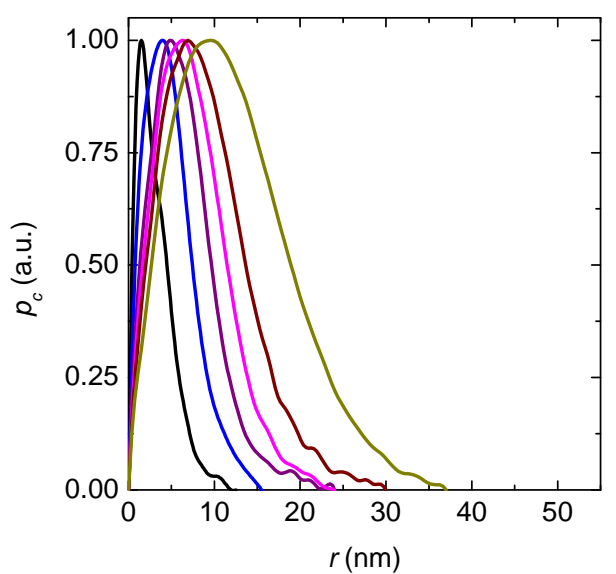

(b) $0 \mathrm{mM} \mathrm{KCl}$

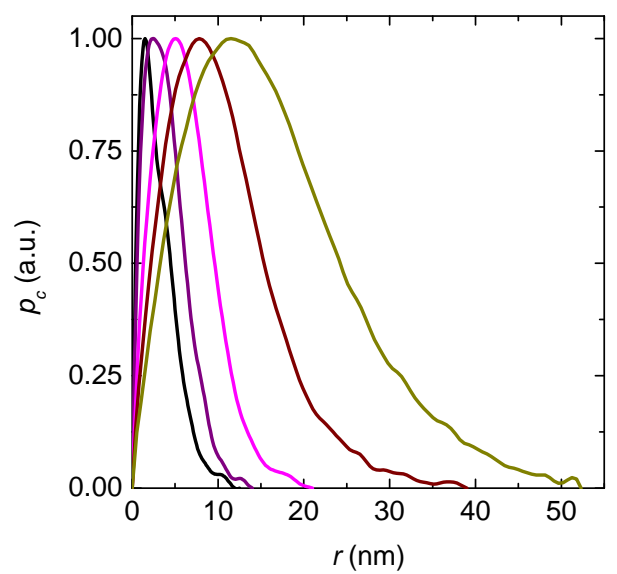

(d) $80 \mathrm{mM} \mathrm{KCl}$

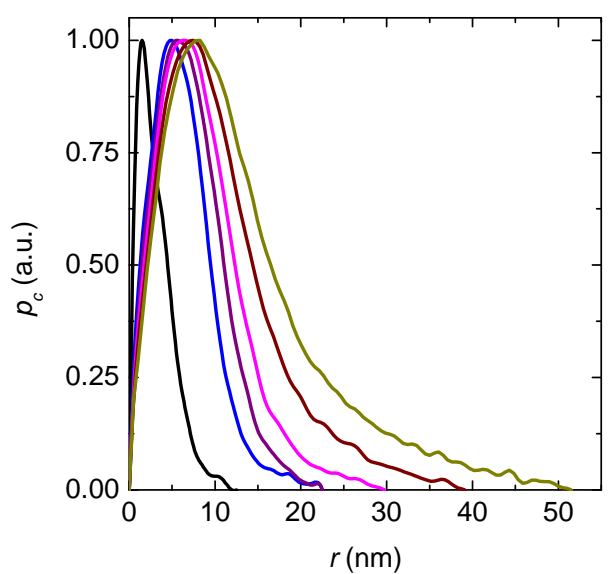

Figure 4.14: Pair distance distribution function of the vimentin wild type at different salt concentrations for (a) increasing potassium chloride concentration in the absence of magnesium chloride (b) increasing magnesium chloride concentration in the absence of potassium chloride (c) increasing magnesium chloride concentration at $20 \mathrm{mM} \mathrm{KCl}$ and (d) increasing magnesium chloride concentration at $80 \mathrm{mM} \mathrm{KCl}$. All curves are normalized to equal peak height. The color code is identical to the figures 4.5 and 4.6: the black curve represents low salt conditions, red, green, blue, cyan and magenta increasing potassium chloride concentration and violet, orange, brown and yellow increasing magnesium chloride concentrations.

to $2.5 \mathrm{~nm}$ with a shoulder at $3.5 \mathrm{~nm}$. At higher magnesium chloride concentrations the peaks are symmetric with no shoulders, they broaden and their position moves to larger distances $\left(11.5 \mathrm{~nm}\right.$ at $\left.4 \mathrm{mM} \mathrm{MgCl}_{2}\right)$. The height and length of the tail 
towards large distances increases with the magnesium chloride concentration. In the presence of $20 \mathrm{mM} \mathrm{KCl}$, the addition of magnesium chloride removes the shoulder at $1.5 \mathrm{~nm}$. The peak moves to larger distances (9.5 $\mathrm{nm}$ for $4 \mathrm{mM} \mathrm{MgCl}_{2}$ ) and broadens. At $1.5 \mathrm{mM}$ and $2.5 \mathrm{mM}$ there is an additional small shoulder at $4.5 \mathrm{~nm}$. At $4 \mathrm{mM} \mathrm{MgCl}$ the peak is rather symmetric. The tails towards larger distances are shorter than in the presence of only one cation species.

In the presence of $80 \mathrm{mM} \mathrm{KCl}$, the position of the peak moves less when magnesium chloride is added, from $4.75 \mathrm{~nm}$ at $0 \mathrm{mM} \mathrm{MgCl}$ to only $7.75 \mathrm{~nm}$ at $4 \mathrm{mM} \mathrm{MgCl}_{2}$. However, the tails of the decay towards zero become noticeably larger with higher magnesium concentration and lie somewhere between those at $0 \mathrm{mM} \mathrm{MgCl}_{2}$ and $20 \mathrm{mM} \mathrm{MgCl}_{2}$.

The structural information contained in the PDDFs provides an alternative approach to estimating the cross-sectional radius of gyration of vimentin at different salt concentrations by determining the second momentum of the PDDF. Further, the calculation of the PDDFs includes an estimate of the forward scattering amplitude. Figure 4.15 gives both the radii of gyration based on the PDDFs and the forward scattering amplitudes from their calculation process for different salt concentrations. We see that both parameters and the salt concentrations dependence are in general comparable to those obtained by the Guinier approximation. The radii of gyration tend to be slightly larger by a factor of about 1.1, and the forward scattering amplitudes are nearly identical. The only deviations are found at $20 \mathrm{mM}$ $\mathrm{KCl}, 0 \mathrm{mM} \mathrm{MgCl} 2$ and at $80 \mathrm{mM} \mathrm{KCl}$ for both $2.5 \mathrm{mM} \mathrm{MgCl}_{2}$ and $4 \mathrm{mM} \mathrm{MgCl}_{2}$. In the first case, the radius of gyration based on the PDDF is most likely overestimated due to the extended, but not necessarily realistic, tail region found in that particular PDDF. The latter two points, however, represent a general trend and because of the reduced $q$-range contributing to the Guinier approximation none of the estimates can be considered more probable than the other.

When we regard the PDDFs of the $Y 117 \mathrm{~L}$ mutant, we see that their peaks at low potassium chloride concentrations are at similar distances as for the wild type. At $100 \mathrm{mM}$ the peak position reaches $7.5 \mathrm{~nm}$ and as for the wild type the peak is rather symmetric with a small tail extruding to larger distances. Interestingly whereas the scattering curves of the wild type and the $Y 117 \mathrm{~L}$ mutant at $10 \mathrm{mM} \mathrm{KCl}$ are very similar (see figure 4.9b), their PDDFs show distinct differences: Instead of just a shoulder at the location of the tetrameric peak, the mutant features a secondary peak. This illustrates how sensitive the PDDF transformation is to the original 
(a) $0 \mathrm{mM} \mathrm{MgCl} 2$

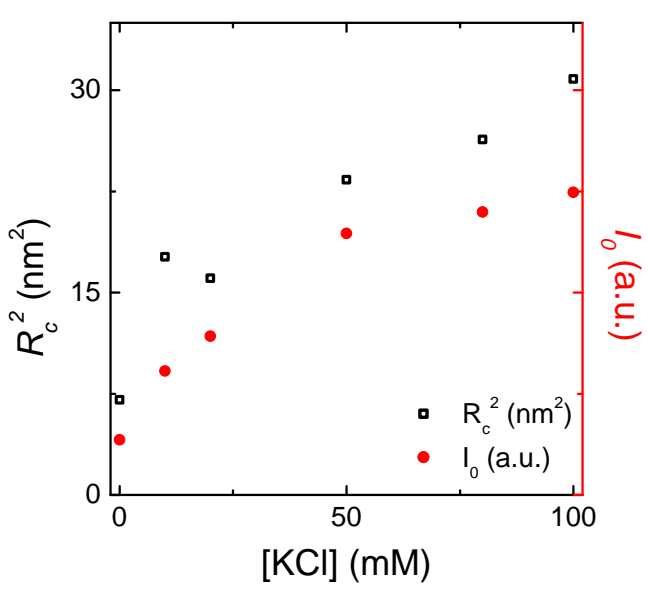

(c) $20 \mathrm{mM} \mathrm{KCl}$

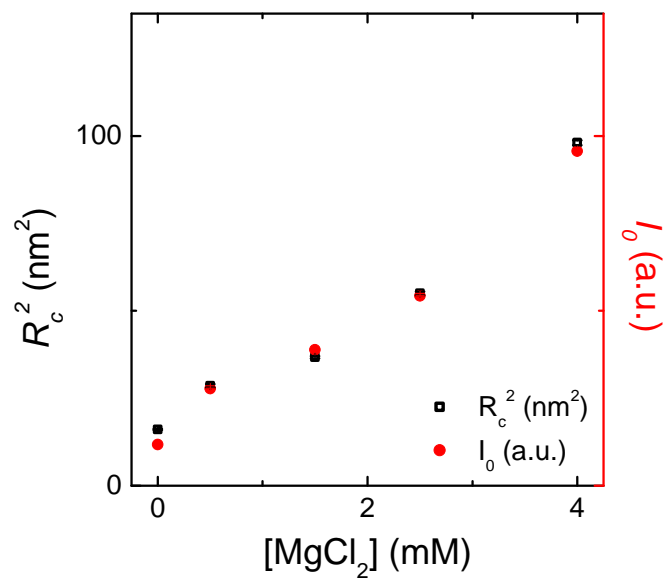

(b) $0 \mathrm{mM} \mathrm{KCl}$

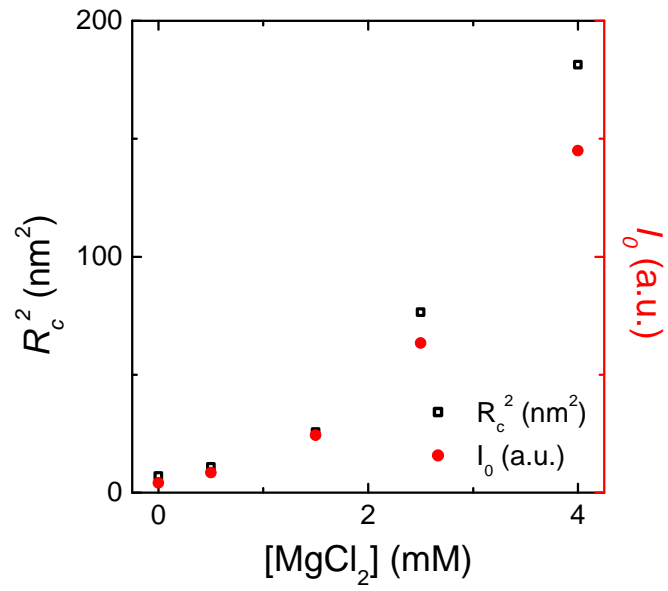

(d) $80 \mathrm{mM} \mathrm{KCl}$

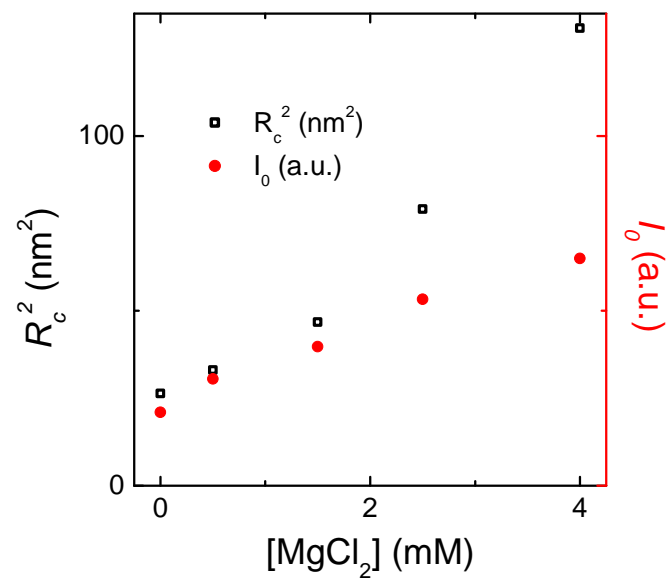

Figure 4.15: Cross-sectional gyration radii and forward scattering amplitude of the vimentin wild type obtained from the PDDFs and their calculation for different salt concentrations. (a) Different potassium chloride concentrations in the absence of magnesium chloride. (b) Different magnesium chloride concentrations in the absence of potassium chloride. (c) Different magnesium chloride concentrations at $20 \mathrm{mM} \mathrm{KCl}$. (d) Different magnesium chloride concentrations at $80 \mathrm{mM} \mathrm{KCl}$. 
(a) $0 \mathrm{mM} \mathrm{MgCl} 2$

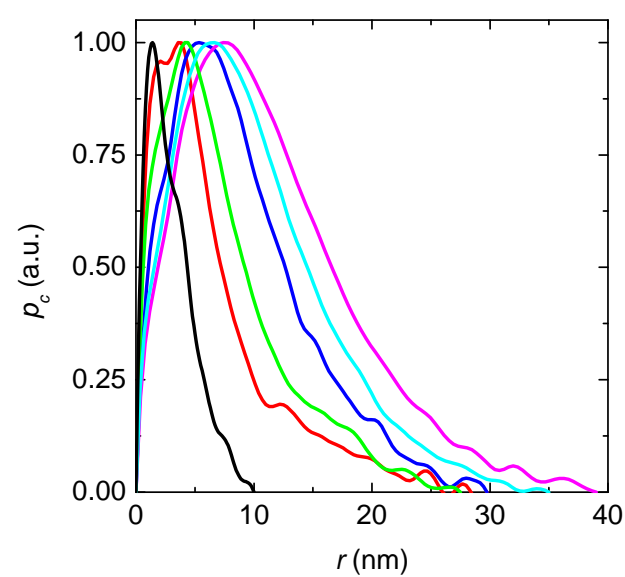

(c) $0 \mathrm{mM} \mathrm{MgCl} 2$

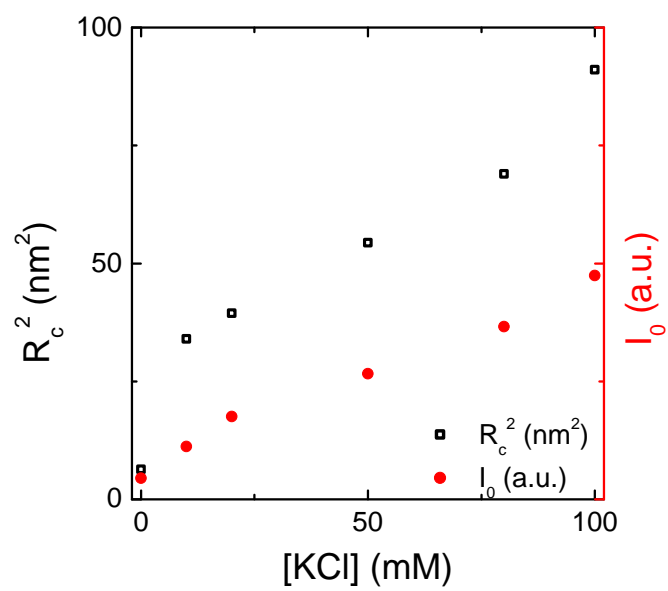

(b) $2.5 \mathrm{mM} \mathrm{MgCl} 2$

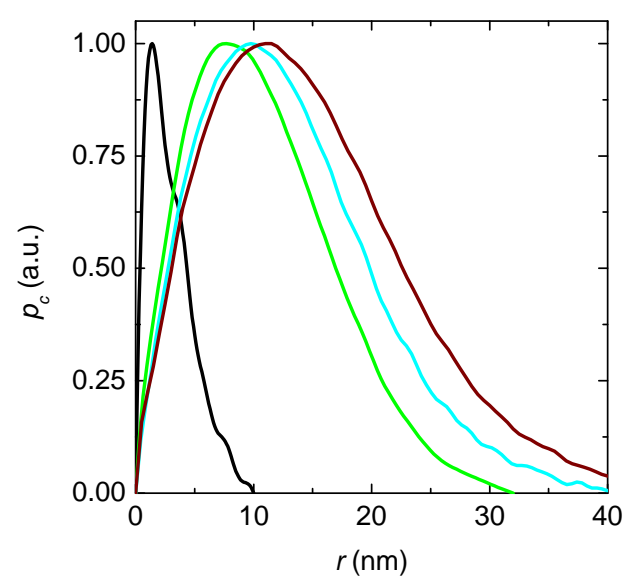

(d) $2.5 \mathrm{mM} \mathrm{MgCl} 2$

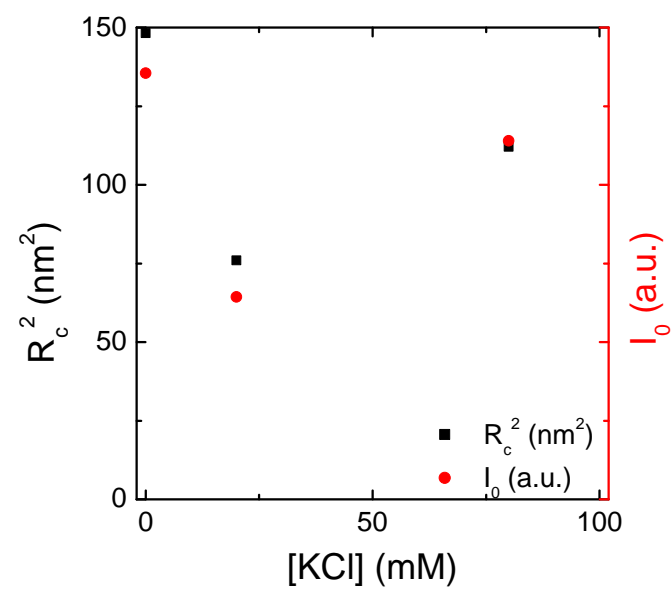

Figure 4.16: (a), (b) Pair distance distribution functions of the $Y 117 \mathrm{~L}$ mutant for different salt concentrations, scaled by the forward scattering amplitude for cross-sectional gyration radii and forward scattering amplitude for increasing potassium chloride concentration at $0 \mathrm{mM} \mathrm{MgCl}$ and $2.5 \mathrm{mM} \mathrm{MgCl}_{2}$, respectively. The color code corresponds to figures 4.7 and 4.8 with the black curve representing low salt concentrations, brown representing $2.5 \mathrm{mM} \mathrm{MgCl} 2$ only, and green, blue cyan and magenta representing increasing potassium chloride concentrations. (c), (d) Cross-sectional radii of gyration and forward scattering amplitudes obtained PDDFs at $0 \mathrm{mM} \mathrm{MgCl}_{2}$ and $2.5 \mathrm{mM} \mathrm{MgCl}_{2}$, respectively. 
curve. In contrast to the wild type, the shoulder at $1.5 \mathrm{~nm}$ can still be clearly recognized at $50 \mathrm{mM} \mathrm{KCl}$.

In the presence of $2.5 \mathrm{mM} \mathrm{MgCl}_{2}$ all three PDDF curves are similarly shaped with the broadest peak at highest distances for $0 \mathrm{mM} \mathrm{KCl}$ and the narrowest peak at smallest distances at $20 \mathrm{mM} \mathrm{KCl}$. None of the curves shows a shoulder at $1.5 \mathrm{~nm}$ (figure 4.16b). When comparing the radii of gyration and the forward scattering amplitudes to those obtained by the Guinier approximation most values, with the exception of the radius of gyration for $10 \mathrm{mM} \mathrm{KCl}$, are again very similar.

To summarize, both the cross-sectional radius of gyration from the Guinier approximation and location of the peaks in the PDDFs show that for the wild type in the presence of only monovalent cations the radius of the filaments depends only slightly on the ion concentration whereas in the presence of only divalent cations, changes in their concentration induce strong changes in the radius. With increasing monovalent ion concentration these changes get smaller. For the mutant monovalent cations induce a strong increase in radial dimensions which does not saturate as for the wild type. At low cation concentrations the PDDFs of both the wild type and the $Y 11^{\prime 2} \mathrm{~L}$ mutant indicate the presence of structure with a cross-section comparable to those of tetramers, possibly corresponding to free tetramers.

\subsubsection{Form factors of structural models}

From the Guinier approximation and the PDDFs we could obtain some clues on general structural changes of the filaments. A different approach to obtain structural information is to build a model based on available information, calculate its form factor and use the form factor to fit the free parameters to the SAXS curve. For vimentin, we know that in the presence of $100 \mathrm{mM} \mathrm{KCl}$ it forms filaments, that are several $\mu \mathrm{m}$ long, with diameters between $8 \mathrm{~nm}$ and $11 \mathrm{~nm}$ with a distribution width of the diameter of $13.4 \%$ [32]. It is also likely that the flexible tail chains are located outside of the central core, see section 2.1.1.

The easiest model to describe an intermediate filament would therefore be a very long cylinder. Based on equations 3.7 - 3.10 the form factor for a cylinder of length $L$ and radius $R$, with both $L \gg R$ and $L q \gg 1$, is

$$
F_{c y l}(q) \propto \frac{1}{q L}\left(\frac{R^{2} B_{1}(q R)}{q R}\right)^{2}
$$


where $B_{1}(x)$ is the first bessel function of the first kind, for details see appendix B.3.

This leaves only three free parameters for fitting: the absolute scaling of the curve (which includes $L$ ), a general offset and the filament radius $R$. As an example, figure 4.17 shows the SAXS data recorded at $80 \mathrm{mM} \mathrm{KCl}$ and the resulting fit for monodisperse cylinders $\left(R=6.2 \mathrm{~nm}\right.$, fit between $0.07 \mathrm{~nm}^{-1}$ and $3 \mathrm{~nm}^{-1}$, orange curve). The overall slope of the fit at high $q$ is too steep and, as the form factor has actual zeros, the minima are too deep. However, it does reproduce the slope before the first minimum and the position of the minima and maxima.

A first extension of this simple model is to add polydispersity to the radius. We model the variation of the diameter with a Gaussian distribution whose width is either kept fixed at $13.4 \%$ of the mean diameter or serves as an additional free parameter.

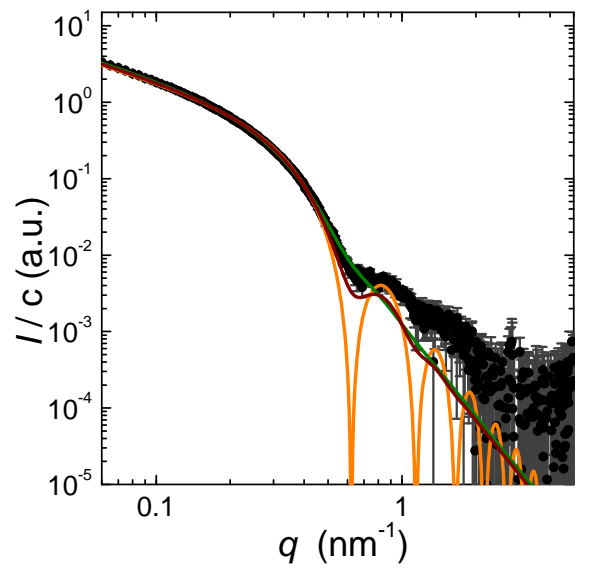

Figure 4.17: Comparison of the SAXS curve of vimentin at $80 \mathrm{mM} \mathrm{KCl}$ (black circles) and fits to different form factors: monodisperse cylinders (orange), polydisperse cylinders with fixed polydispersity (brown) and polydispersity as additional fit parameter (green).

When the polydispersity is kept fixed, both the minima and maxima are smoothed out to a level comparable to the data. However, the slope from about $0.5 \mathrm{~nm}^{-1}$ upwards is too large (brown line in figure 4.17). The resulting mean radius of $6.3 \mathrm{~nm}$ is slightly larger than the radius for monodisperse cylinders but results in a similar slope at small $q$.

When the polydispersity is one of the free parameters of the fit, the width of the Gaussian increases to $21.2 \%$ of the mean radius. This smoothes out all local minima and maxima, indicating that the polydispersity is actually overestimated, but still fails to reproduce the slope above $0.5 \mathrm{~nm}^{-1}$. The resulting mean radius is $6.6 \mathrm{~nm}$ and the slope at small $q$ is again similar to the monodisperse model.

In addition to not reproducing the data, all three fits also overestimate the filament radius which should be in the range of $4-5 \mathrm{~nm}$. The simple rod therefore does not describe vimentin filaments well and we need to consider more complex models. 
From the above, it follows that a cylinder model can describe the SAXS curve at small $q$ but fails at larger $q$. Therefore, any addition to the cylinder model should decay more slowly at high $q$ than the cylinder model itself. One possibility for such an addition is to model the flexible tail regions of vimentin as a corona of Gaussian chains attached to the cylinder, see figure 4.18. The form factor for such a model has been derived by Pedersen [84].

(a)

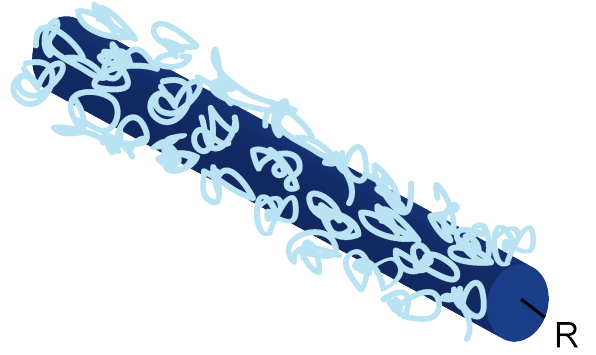

(b)

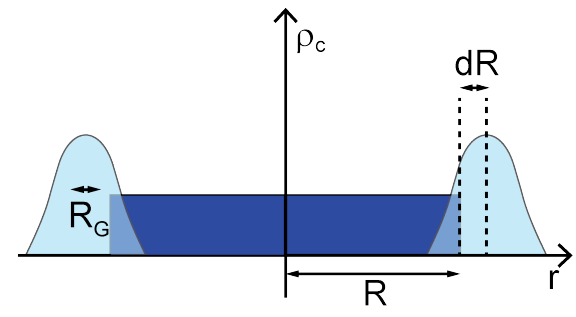

Figure 4.18: (a) Sketch of the model used to describe vimentin filaments: The tail regions form Gaussian chains (light blue) which are attached to a solid cylinder core with radius $R$ (dark blue). (b) The radial electron density of the IF model: The electron density within the central cylinder $(r<R)$ is constant. At a distance $d R$ from the cylinder a Gaussian distribution describes the flexible tail regions. The width of the distribution is related to the radius of gyration via $R_{G}^{2}=\frac{1}{6}\left\langle r^{2}\right\rangle$

In general, the excess electron density $\rho(\vec{r})$ for such a model can be described as $[64,84,85]$ :

$$
\rho(\vec{r})=\rho_{\text {core }}(\vec{r})+\sum_{n=1}^{N_{G}} \rho_{\text {gaussian }}\left(\vec{r}, G_{n}\right) \otimes p_{n}
$$

where $N_{G}$ is the number of Gaussian chains, $\rho_{\text {core }}$ the electron density distribution of the core, $\rho_{\text {gaussian }}$ the electron density distribution of a single Gaussian chain with the respective radius of gyration of the $n$th chain $G_{n} . p_{n}$ describes the position of the chain. The radius of gyration of a Gaussian chain is connected to its average end-to-end distance, via $R_{G}^{2}=\frac{1}{6}\left\langle r^{2}\right\rangle$.

Inserting this relation into equation 3.4 and applying the commutativity, associativ- 
ity and distributivity of the convolution operator, we obtain

$$
\begin{aligned}
I(q)=F(q) & =<\int e^{i \vec{q} \vec{r}}\left[\left(\rho_{\text {core }}+\sum_{1}^{N_{G}} \rho_{\text {gaussian }}\left(G_{n}\right) \otimes p_{n}\right) \otimes\right. \\
& \left.\otimes\left(\rho_{\text {core }}+\sum_{n=1}^{N_{G}} \rho_{\text {gaussian }}\left(G_{n}\right) \otimes p_{n}\right)\right] \mathrm{d}^{3} r>= \\
& =<\int e^{i \vec{q} \vec{r}}\left[\rho_{\text {core }} \otimes \rho_{\text {core }}+\right. \\
& +\sum_{n=1}^{N_{G}} \rho_{\text {gaussian }}\left(G_{n}\right) \otimes p_{n, \text { shell }} \otimes \rho_{\text {gaussian }}\left(G_{n}\right) \otimes p_{n}+ \\
& +\sum_{n=1}^{N_{G}} \sum_{\substack{m=1 \\
m \neq n}}^{N_{G}} \rho_{\text {gaussian }}\left(G_{n}\right) \otimes p_{n} \otimes \rho_{\text {gaussian }}\left(G_{m}\right) \otimes p_{m}+ \\
& \left.+2 \sum_{n=1}^{N_{G}} \rho_{\text {gaussian }}\left(G_{n}\right) \otimes p_{n} \otimes \rho_{\text {core }}(\vec{r})\right] \mathrm{d}^{3} r>\equiv \\
& \equiv I_{s}+I_{d}+I_{\text {cc }}+I_{c s}
\end{aligned}
$$

We therefore have to consider four separate terms: the scattering of the core itself $I_{s}$, the scattering within individual chains $I_{c}$, the scattering between different chains $I_{c c}$ and the contribution of the scattering between core and chains $I_{c s}$.

For a filament of length $L$ and radius $R$, the contribution of the core is equivalent to the scattering from an ideal cylinder, see equations 3.4 and 4.1:

$$
I_{s}(q)=N_{U L F} \beta_{s}^{2} P_{L}(q, L)\left(\frac{2 B_{1}(q R)}{q R}\right)^{2}
$$

where $N_{U L F}$ is the number of ULFs in the filament and $\beta_{s}$ is the forward scattering of the core per ULF.

Further, all tails in a vimentin filament should have the same radius of gyration, i.e. $G_{n}=R_{G}$. Therefore

$$
I_{c}=N_{G} \beta_{c}^{2} \frac{2\left(\exp \left(-\left(q R_{G}\right)^{2}\right)-1+\left(q R_{G}\right)^{2}\right)}{\left(q R_{G}\right)^{4}} \equiv N_{G} \beta_{c}^{2} F_{d}(q)
$$

which corresponds to the scattering of a single Gaussian chain as given by Debye [86] with an individual forward scattering amplitude $\beta_{c}$.

Additionally, all tails are located at the distance $d R$ from the cylinder surface on 
the same shell around the cylinder which in cylinder coordinates $(z, r, \theta)$ is given by

$$
p_{n}(\vec{r})=\Theta(z-L / 2) \Theta(L / 2-z) \delta(R+d R-r)
$$

where the step function $\Theta$ and the $\delta$ function are normalized such that the volume integral over $p_{n}$ is 1 . As

$$
<\int e^{i \vec{q} \vec{r}} \delta(R+d R-r) d^{2} r>\propto B_{0}(q(R+d R))
$$

(see appendix B.3), the cross-term between the core and the chains becomes

$$
I_{c s}(q)=2 N_{U L F} N_{G} \beta_{s} \beta_{c} \Psi\left(q R_{G}\right) \frac{2 B_{1}(q R)}{q R} B_{0}(q(R+d R)) F_{L}(q, L)
$$

where $\Psi\left(q R_{G}\right)=\frac{1-\exp \left(-\left(q R_{G}\right)^{2}\right)}{\left(q R_{G}\right)^{2}}$ is the approximative form factor amplitude of a Gaussian chain [87] and $B_{0}$ the zeroth order Bessel function of the first kind.

Finally, the scattering between different chains in the corona is given by

$$
I_{c c}(q)=N_{G}\left(N_{G}-1\right) \beta_{c}^{2} \Psi\left(q R_{G}\right)^{2} B_{0}(q(R+d R))^{2} F_{L}(q, L)
$$

As both the length of intermediate filaments and their persistence length are significantly longer $(\mu \mathrm{m})$ than the structural range accessible by the recorded $q$-range [11] we can approximate

$$
F_{L}=\frac{\pi}{q L}
$$

for the longitudinal contributions [69, 72,73, 88].

We can reduce the number of independent parameters in the form factor by using the facts that

i) the length of a filament $L$ is linear in the number of ULFs $N_{U L F}\left(L \approx l N_{U L F}\right.$, where $l$ is about $43 \mathrm{~nm}$ ) [48]

ii) the number of Gaussian chains per ULF is constant with $N_{G}=n N_{U L F}$, as a ULF consists of a fixed number of vimentin monomers [23]

iii) for $N_{G} \gg 1$ additionally $N_{G}\left(N_{G}-1\right) \approx N_{G}^{2}$

Hence applying

$$
F_{L} N_{G}^{2}=\frac{\pi}{q L} N_{G}^{2}=\frac{\pi}{q l N_{U L F}} N_{G}^{2}=n^{2} N_{U L F} \frac{\pi}{q l}
$$


the complete form factor becomes linear in $N_{U L F}$ :

$$
\begin{aligned}
F_{F}(q)= & N_{U L F} \gamma\left[\frac{\pi}{q}\left(\frac{2 B_{1}(q R)}{q R}\right)^{2}+\lambda b^{2} F_{c}(q)+\right. \\
& +2 \cdot b \frac{\pi}{q} \Psi\left(q R_{G}\right) \frac{2 B_{1}(q R)}{q R} B_{0}(q(R+d R))+ \\
& \left.+b^{2} \frac{\pi}{q} \Psi\left(q R_{G}\right)^{2} B_{0}(q(R+d R))^{2}\right]
\end{aligned}
$$

where we introduced $\gamma=\beta_{s}^{2} / l$, the forward scattering amplitude per unit length of a $\mathrm{ULF}, \lambda=l / n$, as the average distance between chains along the rod, and $b=n \beta_{c} / \beta_{s}$, as the scattering ratio of the side chains of an ULF to the core, reducing the number of independent parameters to seven. Hence, for an ensemble of filaments of different length, the scattering does not depend on the length distribution of filaments but only on the total protein concentration (see equation 3.12).

Figure 4.19 shows two exemplary fits to equation 4.9. We see that in both cases

(a) $80 \mathrm{mM} \mathrm{KCl}$

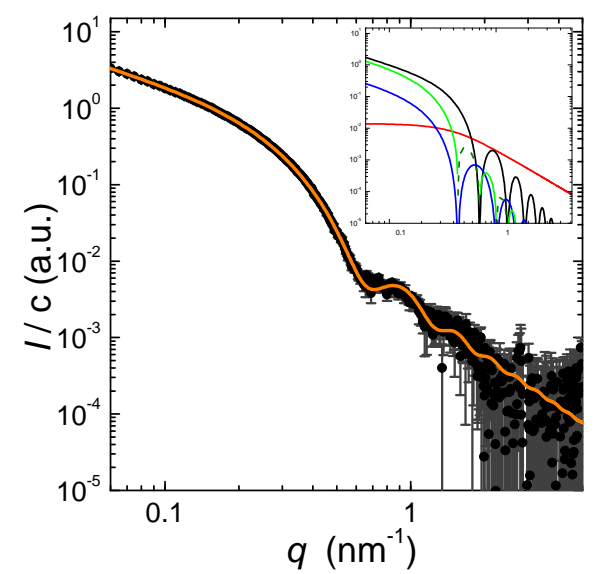

(b) $4 \mathrm{mM} \mathrm{MgCl} 2$

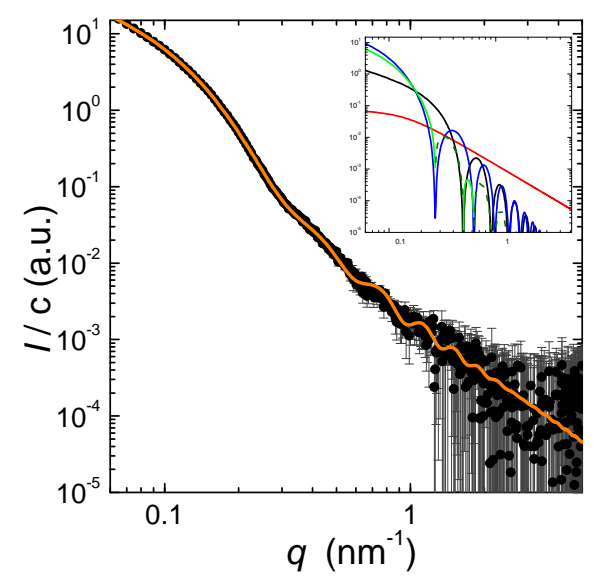

Figure 4.19: Fits of SAXS curves (black circles) to equation 4.9 (orange lines) (a) $80 \mathrm{mM} \mathrm{KCl}$ and (b) $2.5 \mathrm{mM} \mathrm{MgCl} 2$. The insets show the individual components: the core scattering $I_{s}$ (black), the scattering in individual side chains $I_{c}$ (red), the scattering between different side chains $I_{c c}$ (blue) and the scattering between side-chains and rod, $I_{c s}$ (green) and $-I_{c s}$ (olive). In both cases the fits describe the general shape well, but overestimate local minima and maxima.

the fit reproduces the general form of the curves well. However, starting from $q \approx 0.6 \mathrm{~nm}^{-1}$ the fits show local maxima and minima that are either much less prominent or even absent in the data curves. For $80 \mathrm{mM} \mathrm{KCl}$, we obtain a cylinder radius of $5.5 \mathrm{~nm}$, which is smaller than the radius obtained for perfect cylinders, 
but closer to the expected value of about $5 \mathrm{~nm}$ (see section 2.1.1), and a radius of gyration of the tail of $3.6 \mathrm{~nm}$. For $2.5 \mathrm{mM} \mathrm{MgCl}_{2}$, the resulting parameters are $R$ $=7.7 \mathrm{~nm}$ and $R_{G}=10.5 \mathrm{~nm}$.

When we compare the high $q$ behaviour, we see that in both cases it is dominated by the scattering from individual side chains (see inlets in figure 4.19). In contrast, at low $q$ the scattering in the presence of potassium chloride only, is dominated by the rod scattering whereas in the presence of magnesium chloride it is dominated by the cross-terms. The lack of a region where the cross-terms contribute significantly in the abscence of magnesium chloride complicates the determination of the cross-factors, as the resulting fit curve depends on $\lambda b^{2}$ rather than to $\lambda$ and $b^{2}$. Therefore, in these cases it is necessary to keep one of them fixed to obtain results that are comparable between the different salt concentrations for the other one. As we know the length of a ULF and the number of side chains per ULF for the wild type protein in the presence of only potassium chloride, we can keep $\lambda=1.34 \mathrm{~nm}$, corresponding to 32 tails on a $43 \mathrm{~nm}$ long ULF [48]. For higher magnesium chloride concentrations, and for the mutant also for potassium chloride concentrations above $20 \mathrm{mM}$, however, this approach fails to describe the curves, see figure 4.20. This is not surprising, as there is no reason for the number of monomers per ULF to be constant. Rather, it is reasonable to assume that with increasing radius the number of monomers also increases and therefore $\lambda$ decreases.

To reduce the local minima and maxima in the fit curve and to closer approximate our model to reality, we add radial polydispersity. We estimate the standard deviation of the filament thickness distribution to $13.4 \%$ of the average radius [32]. As both the polydispersity of the core and the flexibility of the corona might contribute to this polydispersity observed by TEM, this is an upper limit to the polydispersity of the core only. Test fits with the radial polydispersity as a free parameter result in nearly identical fit curves and radial polydispersities of the same order as $13.4 \%$. We further assume that all pre-factors are monodisperse for a given ionic composition which is equivalent to assuming that the number of monomers per ULF is monodisperse. As the fits return $d R / R_{G} \ll 1$ and all other parameters are physically realistic, we decide to limit the free parameter space by setting $d R=0$. For the same reasons, we set the global offset to 0 . Further, for $10 \mathrm{mM}$ and $20 \mathrm{mM}$ potassium chloride, estimates of the radius tend to be smaller than $3 \mathrm{~nm}$, which is unrealistic considering the radius of tetramers. This issue can be easily resolved by assuming that not all tetramers are built into filaments, which is reasonable, 
considering the PDDFs. We therefore fit the data to

$$
F_{T}(q)=(1-t) \cdot F_{F}(q)+t \cdot I_{t e t}(q)
$$

where $F_{F}$ is the polydisperse extension of equation $4.9, I_{\text {tet }}$ the recorded tetrameric SAXS curve for the corresponding protein concentration and $t$ the ratio of tetramers in the sample. The $q$-range for the fits was set to $0.07 \mathrm{~nm}^{-1}$ to $3 \mathrm{~nm}^{-1}$.

Figure 4.20 shows exemplary fits to equation 4.10 for two different ionic composi-

(a) $80 \mathrm{mM} \mathrm{KCl}$

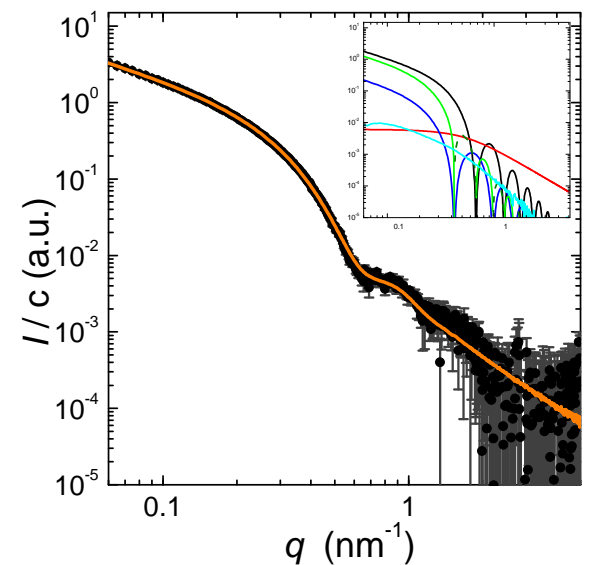

(b) $4 \mathrm{mM} \mathrm{MgCl} 2$

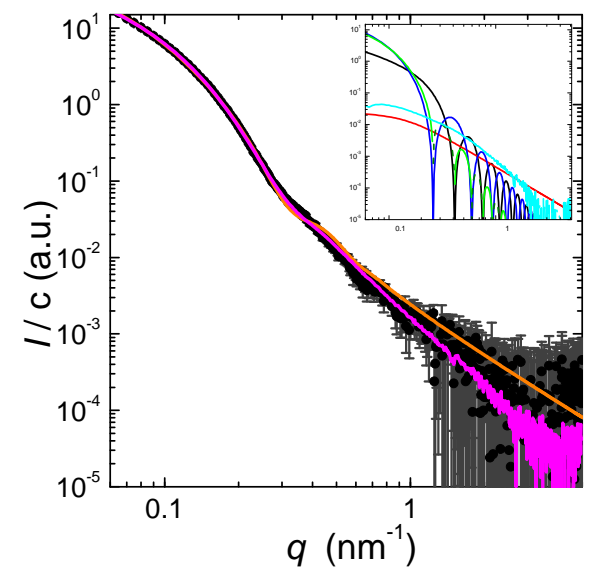

Figure 4.20: Fits of SAXS curves (black dots) to equation 4.10 with $\lambda$ fixed (orange lines) and not fixed (magenta line) for (a) $80 \mathrm{mM} \mathrm{KCl}$ and (b) $2.5 \mathrm{mM} \mathrm{MgCl}$. The insets show the individual components: the core scattering $I_{s}$ (black), the scattering in individual side chains $I_{c}$ (red), the scattering between different side chains $I_{c c}$ (blue) and the scattering between side-chains and rod, $I_{c s}$ (green) and $-I_{c s}$ (olive), in (b) for the case that $\lambda$ is a free parameter, i.e. corresponding to the magenta line.

tions. In the presence of $80 \mathrm{mM} \mathrm{KCl}$, the fit with $\lambda$ fixed describes the curve well and always lies within the error bars, but underestimates the second local maximum. Indeed, even a broader scan of the parameter space returns no fits which scale the first and second local maximum correctly to each other, possibly indicating that the solid cylindrical core might not be a perfect description (see section 4.5.2). In contrast, in the presence of $4 \mathrm{mM} \mathrm{MgCl}_{2}$, keeping $\lambda$ fixed results in a local minimum/maximum pair at about $0.4 \mathrm{~nm}^{-1}$ which is not present in the data. Additionally, the fit in this region lies outside the error bar. Using $\lambda$ as a free parameter however, leads to a very good description of the curve. When we compare the contributions of individual components, we see that the local minima and maxima observed in the presence of only potassium chloride are mostly caused by scattering from within the 
cylindrical core, whereas the kinks observed in the presence of magnesium chloride have a major contribution from the scattering of the corona and are therefore less distinct and less directly linked to real-space structure.

Analogously, we fit the SAXS curves for tetrameric vimentin to this model, assuming that head and tail regions sum up to a total of eight Gaussian chains and that the length of a tetramer is $63 \mathrm{~nm}$. The $q$-range for this fit is reduced to $0.15 \mathrm{~nm}^{-1}$ to $3 \mathrm{~nm}^{-1}$.

Having established fits that describe the scattering curves well and that are based on previous knowledge, we can now discuss how the individual parameters of the fit - and the corresponding real-space features - depend on the ionic composition. To begin, we consider the changes in the amount of vimentin present in tetrameric form by comparing the contribution $t$ of the tetrameric SAXS signal to the overall curves (figure 4.21). At $10 \mathrm{mM}$ and $20 \mathrm{mM} \mathrm{KCl}$, the tetrameric component of wild type vimentin remains quite high $-55 \%$ and $35 \%$ respectively. For higher potassium chloride concentrations, it reduces to below $10 \%$. In contrast, for the $Y 11^{2} \mathrm{~L}$ mutant this reduction is slower with a tetrameric component of about $25 \%$ even at $50 \mathrm{mM} \mathrm{KCl}$. For higher concentrations, it also reduces to below $10 \%$. Increasing the magnesium chloride concentration (either with or without potassium present) also leads to small tetrameric components for the wild type. Only at $0.5 \mathrm{mM} \mathrm{MgCl} 2$ without any potassium more than $50 \%$ of the vimentin remain in tetrameric form. Similarly, for the $Y 11^{7} \mathrm{~L}$ mutant at $2.5 \mathrm{mM} \mathrm{MgCl}_{2}$ only very little vimentin remains tetrameric, independently of the potassium concentration. When we compare these results to the scattering curves in figures 4.5 to 4.7 , we note that the decrease in the tetrameric component correlates with the increase of the scattering at low $q$ : The strongest increase of scattering at low $q$ occures between in the same salt concentration ranges as the decrease of the tetrameric component. This is not surprising, as the scattering of the tetrameric component at low $q$ is much smaller than those of filaments formed at higher salt concentrations and hence the scattering at low $q$ does depend on the amount of tetrameric vimentin present. Due to the fact that the scattering at high $q$ remains nearly constants, this observation also indicates a correlation between the tetrameric component and the overall steepness of the curve.

Next, we can understand how changes in the ionic environment affect the structure of the filaments, i.e. the radius of the cylindrical core and the radius of gyration of the corona. Figure 4.22a shows how these values depend on the potassium chloride concentration for wild type vimentin. We see that at $10 \mathrm{mM} \mathrm{KCl}$ the core radius 
(a) wild type

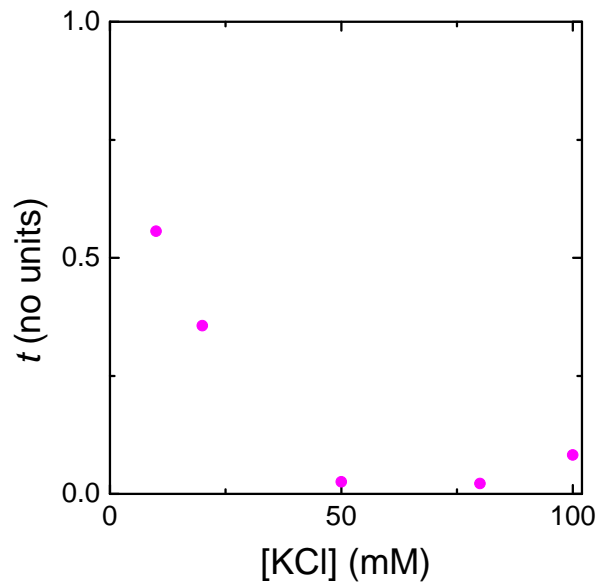

(b) wild type

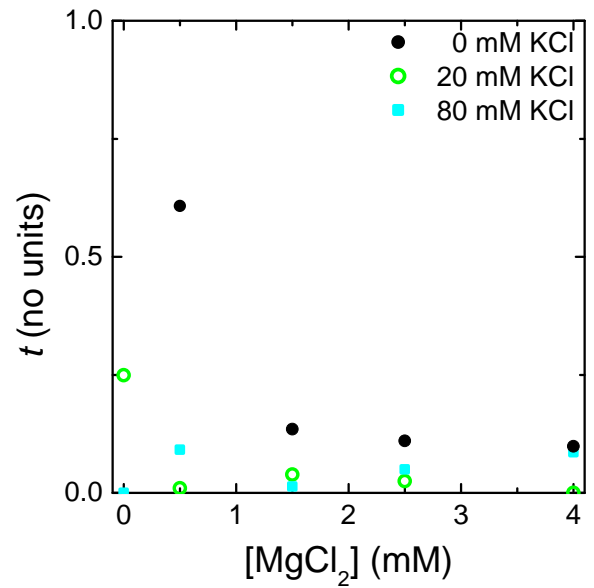

(c) $Y 117 \mathrm{~L}$

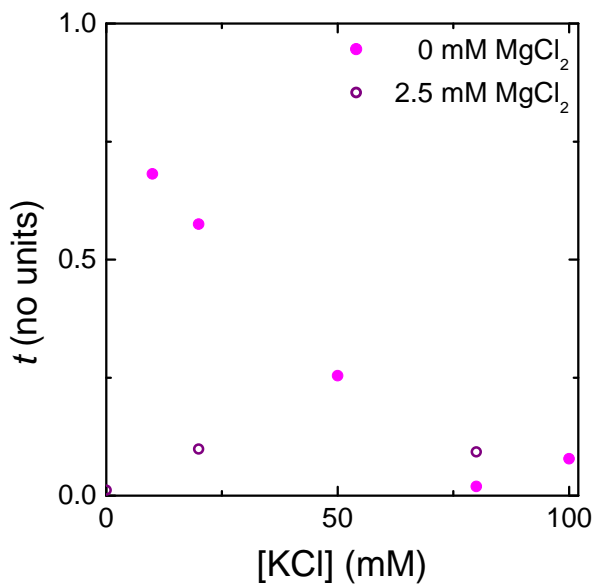

Figure 4.21: Contribution of tetrameric vimentin to the scattering signal at different salt concentrations as estimated by fits to equation 4.10. (a) The addition of potassium chloride to vimentin wild type protein reduces the amount of vimentin in tetrameric form. Above $50 \mathrm{mM} \mathrm{KCl}$, most vimentin is assembled into filaments. (b) Magnesium chloride also reduces the amount of tetrameric vimentin, however at low concentrations and in the absence of potassium, most vimentin remains tetrameric. (c) In the abscence of magnesium chloride, it requires more potassium chloride to reduce the tetrameric form of $Y 117 \mathrm{~L}$. In the presence of $2.5 \mathrm{mM} \mathrm{MgCl}_{2}$ the tetrameric component is consistently small. 
(a) $0 \mathrm{mM} \mathrm{MgCl} 2$

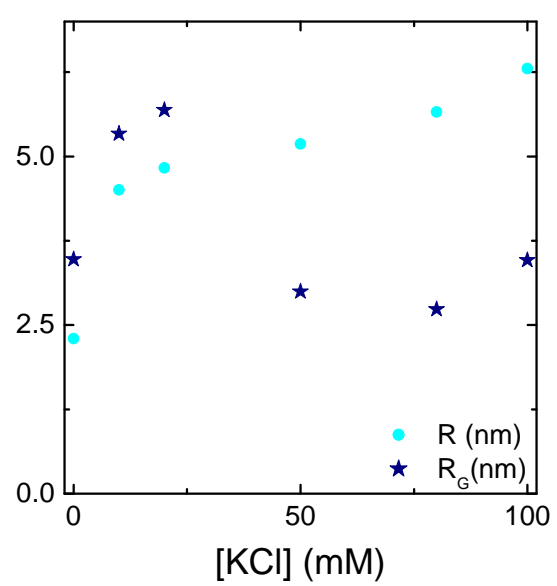

(b) $0 \mathrm{mM} \mathrm{KCl}$

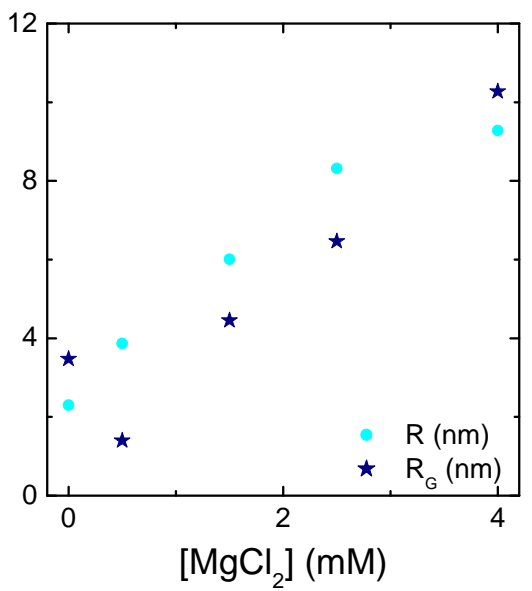

Figure 4.22: Radii of the core cylinder and radii of gyration of the corona as obtained from fits to equation 4.10 for wild type vimentin in the case of (a) different potassium chloride concentrations in the abscence of magnesium chloride and (b) different magnesium chloride concentrations in the abscence of potassium chloride. Changes induced by magnesium are stronger than those caused by potassium.

increases from $2.3 \mathrm{~nm}$ to $4.5 \mathrm{~nm}$ and the radius of gyration increases from $3.4 \mathrm{~nm}$ to $5.3 \mathrm{~nm}$. The radius continues to increase and reaches $6.3 \mathrm{~nm}$ at $100 \mathrm{mM}$ whereas the radius of gyration returns to about $3 \mathrm{~nm}$ from $50 \mathrm{mM}$ on. In contrast, the core radius changes much more strongly between different magnesium chloride concentrations: from $3.9 \mathrm{~nm}$ at $0.5 \mathrm{mM}$ to $9.3 \mathrm{~nm}$ at $4 \mathrm{mM}$. This is accompanied by a continuous increase in the radius of gyration of the corona from $1.4 \mathrm{~nm}$ to $10.3 \mathrm{~nm}$ (figure $4.22 \mathrm{~b}$ ). Adding potassium chloride reduces the effects of increasing the magnesium chloride concentration: At $20 \mathrm{mM} \mathrm{KCl}$ (figure 4.23a) the radius increases from $4.8 \mathrm{~nm}$ to only $8 \mathrm{~nm}$ and the radius of gyration actually reduces to less than $5 \mathrm{~nm}$ before rising to $7.9 \mathrm{~nm}$ at $4 \mathrm{mM} \mathrm{MgCl}_{2}$. At $80 \mathrm{mM} \mathrm{KCl}$, the difference in radius is similar going from $5.7 \mathrm{~nm}$ to $8.3 \mathrm{~nm}$. In contrast, the increase in radius of gyration is more pronounced from $2.7 \mathrm{~nm}$ to $9.2 \mathrm{~nm}$, with most of the increase between $0 \mathrm{mM}$ and $0.5 \mathrm{mM} \mathrm{MgCl}{ }_{2}$.

For the $Y 117 \mathrm{~L}$ mutant, in the abscence of magnesium chloride, the radius increases from $2.2 \mathrm{~nm}$ to $7 \mathrm{~nm}$, i.e. it becomes larger than for the wild type. Concurrently, the radius of gyration increases from $3.6 \mathrm{~nm}$ to about $7.5 \mathrm{~nm}$ already at $10 \mathrm{mM} \mathrm{KCl}$ and remains at this value. At $2.5 \mathrm{mM} \mathrm{MgCl}_{2}$ in the abscence of potassium chloride the radius reaches $9 \mathrm{~nm}$ and the radius of gyration nearly $10 \mathrm{~nm}$, i.e. similar values to the wild type. In the presence of potassium chloride both values reduce. At $20 \mathrm{mM}$ 
(a) $20 \mathrm{mM} \mathrm{KCl}$

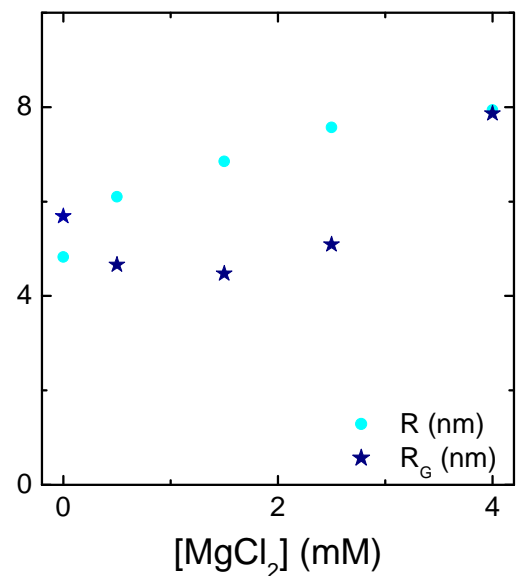

(b) $80 \mathrm{mM} \mathrm{KCl}$

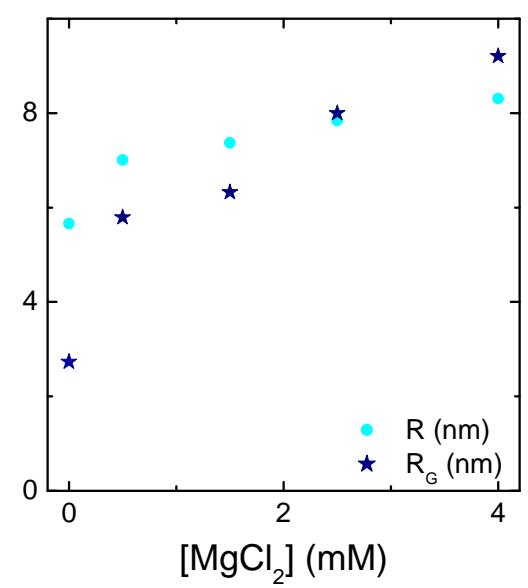

Figure 4.23: Radii of the core cylinder and radii of gyration of the corona as obtained from fits to equation 4.10 for wild type vimentin at different magnesium chloride concentrations in the presence of (a) $20 \mathrm{mM} \mathrm{KCl}$ and (b) $80 \mathrm{mM} \mathrm{KCl}$. At $80 \mathrm{mM} \mathrm{KCl}$ the radius increases less strongly but the change in the radius of gyration is more pronounced.

(a) $0 \mathrm{mM} \mathrm{MgCl} 2$

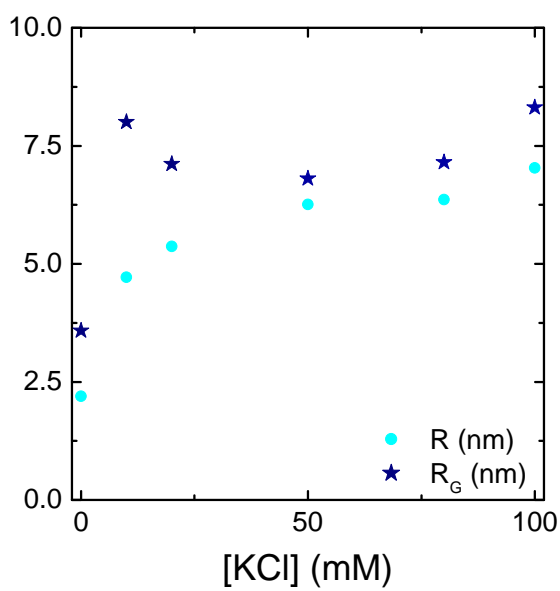

(b) $2.5 \mathrm{mM} \mathrm{MgCl} l_{2}$

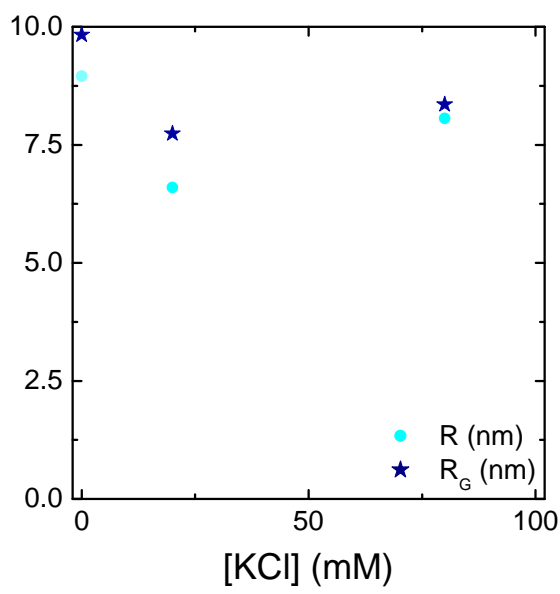

Figure 4.24: Radii of the core cylinder and radii of gyration of the corona as obtained from fits to equation 4.10 for the $Y 17^{r} / \mathrm{L}$ mutant at different potassium chloride concentrations in the presence of (a) $0 \mathrm{mM} \mathrm{MgCl}_{2}$ and (b) $2.5 \mathrm{mM} \mathrm{MgCl}_{2}$. The radii are similar to the wild type but the radii of gyration are larger. 
$\mathrm{KCl}$, the radius is smaller than for the wild type and the radius of gyration larger. At $80 \mathrm{mM} \mathrm{KCl}$, both values are very similar to the wild type.

Finally, let us consider how the contributions of the different components to the scattering change. For this, we consider the forward scattering of the central rod, $N_{U L F} \gamma$, and the ratio of the forward scattering ratio of the side chains to the core, $b$. The insets in figures 4.19 and 4.20 illustrate that the form of the curves in the mid- $q$ range is governed by how the individual components scale in respect to each other and therefore to some extent characterized by $b$.

For the vimentin wild type, with increasing potassium concentration, in the ab-

(a) $0 \mathrm{mM} \mathrm{MgCl} 2$

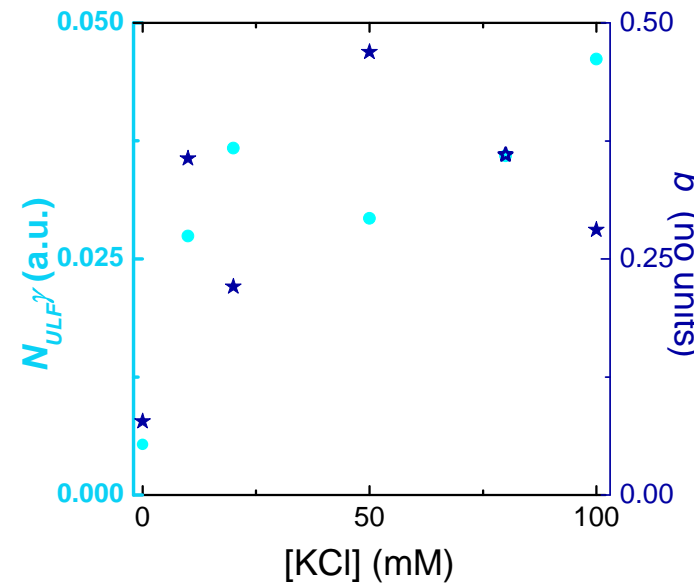

(b) $0 \mathrm{mM} \mathrm{KCl}$

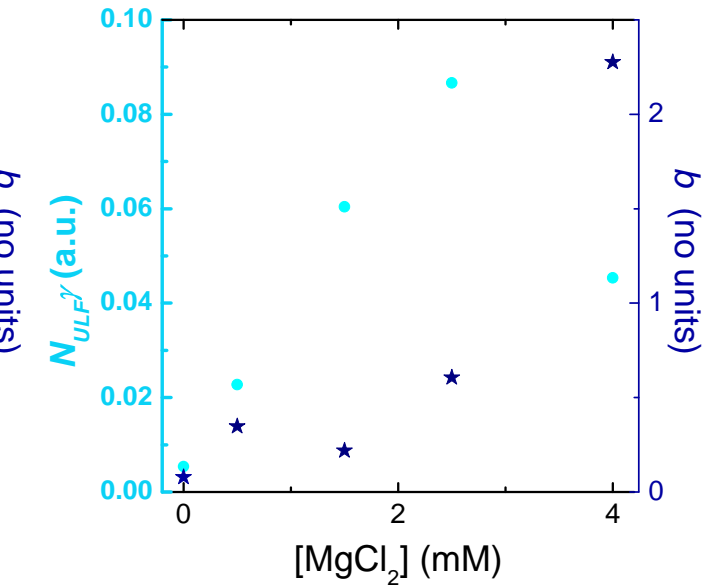

Figure 4.25: Scaling factors of the core and intra-chain scattering for wild type vimentin in dependence of the (a) potassium chloride concentration in the absence of magnesium chloride and (b) magnesium chloride concentration in the absence of potassium chloride.

sence of magnesium, the forward scattering from the rod increases by a factor of a little less than two from $10 \mathrm{mM}$ to $100 \mathrm{mM}$ and $b$ varies between 0.22 and 0.47 with no obvious trend. From $0.5 \mathrm{mM}$ to $2.5 \mathrm{mM} \mathrm{MgCl}_{2}$, the scattering from the rod increases by a factor of 4 while forward scattering of the side chains increases from 0.35 to 0.64 . At $4 \mathrm{mM}$ however, the scattering from the corona decreases by $50 \%$ whereas the contribution from the corona increases to nearly 2.4. Increasing the magnesium chloride concentration at $20 \mathrm{mM}$ potassium choride results in a similar dependence, but the scattering from the side chains increases continuously from 0.2 at $0.5 \mathrm{mM} \mathrm{MgCl}_{2}$ to 2.0 at $4 \mathrm{mM} \mathrm{MgCl} 2$. At $80 \mathrm{mM} \mathrm{KCl}$, the scattering from the rod stays mostly constant between $0.5 \mathrm{mM}$ and $4 \mathrm{mM} \mathrm{MgCl}_{2}$ and the contribution from the chain scattering rises steadily from about 0.18 to about 0.68 of the rod scattering. 
(a) $20 \mathrm{mM} \mathrm{KCl}$

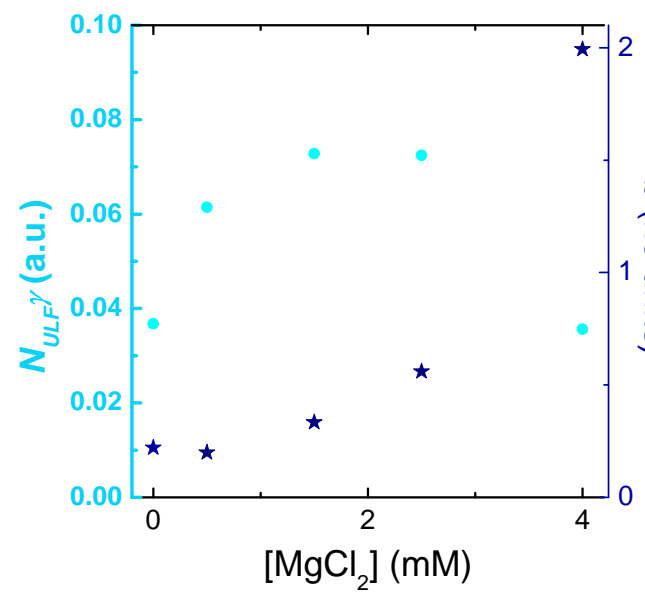

(b) $80 \mathrm{mM} \mathrm{KCl}$

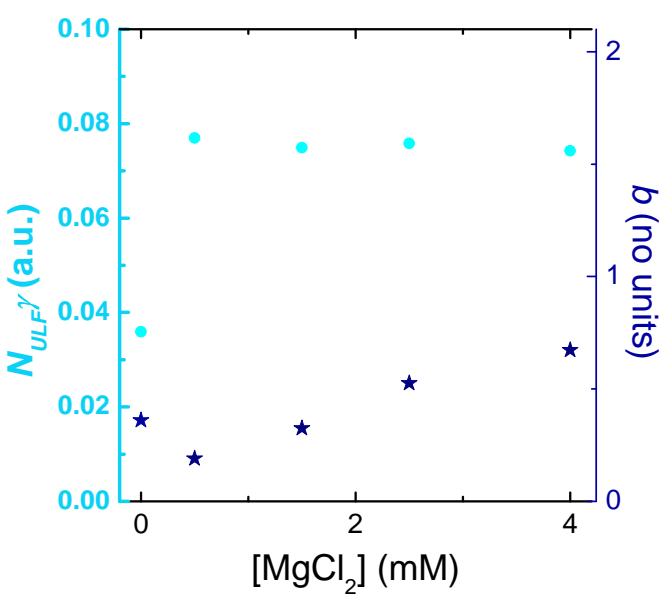

Figure 4.26: Scaling factors of the core and intra-chain scattering for wild type vimentin in dependence of the magnesium chloride concentration at (a) $20 \mathrm{mM} \mathrm{KCl}$ and at (b) $80 \mathrm{mM} \mathrm{KCl}$.

(a) $0 \mathrm{mM} \mathrm{MgCl} 2$

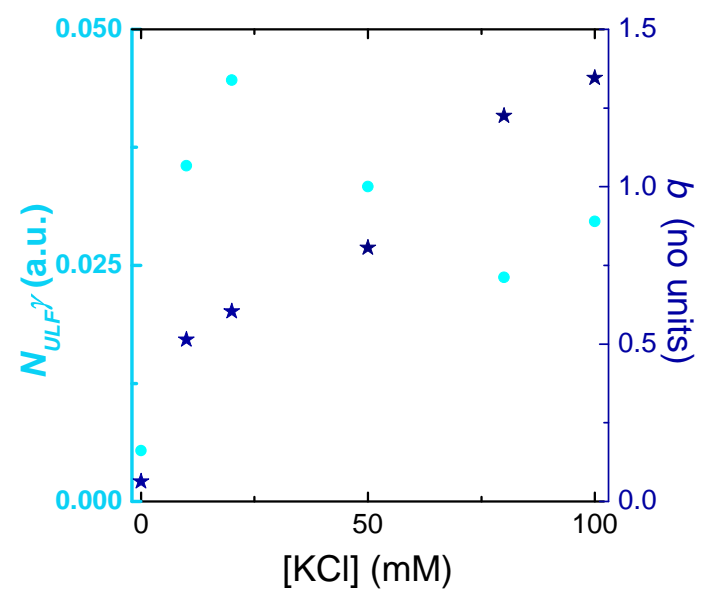

(b) $2.5 \mathrm{mM} \mathrm{MgCl} l_{2}$

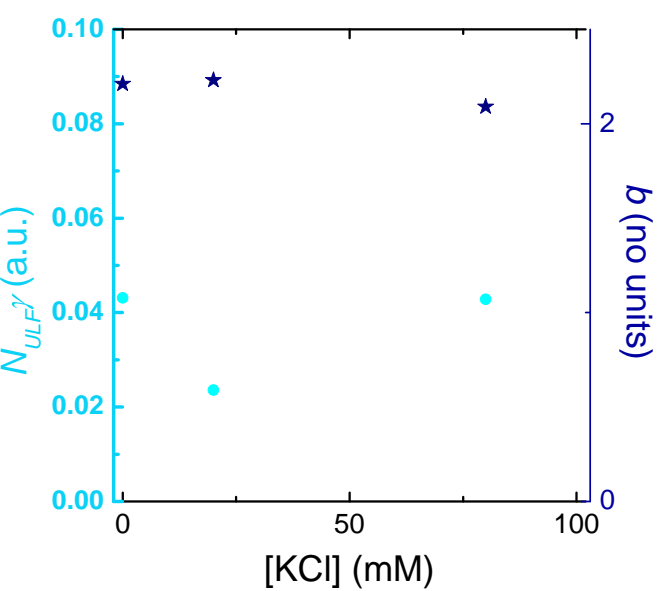

Figure 4.27: Scaling factors of the core and intra-chain scattering for the $Y 117 L$ mutant in dependence of the potassium chloride concentration at (a) at $0 \mathrm{mM}$ and at (b) $2.5 \mathrm{mM}$ magnesium chloride concentration in the absence of potassium chloride. 
For the $Y 117 \mathrm{~L}$ mutant, the trends in the scattering from the core are somewhat similar to those observed for the wild type. The chain scattering however increases nearly linearly from 0.51 at $10 \mathrm{mM} \mathrm{KCl}$ to 1.45 at $100 \mathrm{mM} \mathrm{KCl}$. As the radius and cross-sectional radius of gyration only increase slightly between $50 \mathrm{mM} \mathrm{KCl}$ and $100 \mathrm{mM} \mathrm{KCl}$ (figure 4.24b) the increase of $b$ is one of the most likely contibutors to the emergence of the S-like shape of the scattering curve. In the presence of magnesium chloride, the scattering from the core is always less than for the wild type, and the chain scattering stays nearly constant at 2.2 .

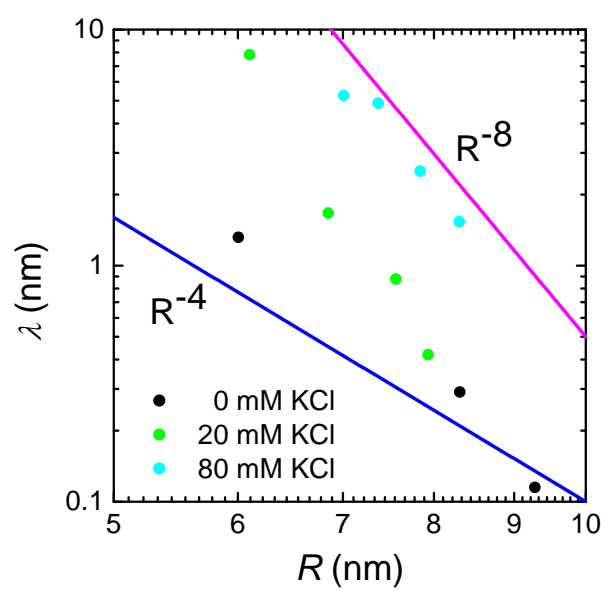

Figure 4.28: The average distance between two chains in the corona $\lambda$ versus the core radius $R$ for different salt concentrations. $\lambda$ deforming the filament cross-section. As we see in the figure, for a fixed potassium chloride concentration, it decreases with the radius. pends on the number of vimentin monomers

When considering the scattering from the corona, it is also important to consider how many chains actually contribute to the corona. The average distance between two chains in the corona $\lambda$ corresponds to the inverse number density of chains along the filament and depends on the ionic environment. It is straightforward that $\lambda$ is connected to the number of vimentin monomers contributing to the filament cross-section and as each monomer has a finite size, $\lambda$ and the filament radius $R$ should be correlated. Therefore we plot $\lambda$ over $R$ for varying magnesium chloride concentrations at different potassium chloride concentrations and see that indeed for a fixed potassium concentration $\lambda$ decreases with increasing radius. Interestingly, $\lambda$ appears to decay faster than $R^{-4}$ but slower than $R^{-8}$.

For the $Y 117 L$ mutant, $\lambda$ shows no distinct trends when the potassium concentration in increased and it is typically in the order of $2 \mathrm{~nm}$. In the presence of magnesium chloride it decreases to the order of $0.5 \mathrm{~nm}$ (no data plot).

Finally, we can compute the cross-sectional radius of gyration for the ensemble of filaments via

$$
R_{c}^{2}=t R_{c}^{2}(\text { tetramer })+\frac{1-t}{1+b}\left((0.5+b) R^{2}+2 b R d R+b R_{G}^{2}\right)
$$


This relation follows from the following considerations

i) The mean squared radius of gyration of a multi-component system is equal to the weighted sum of the squared radii of gyration of the individual components.

ii) For filaments, the cross-sectional radii of gyration can be calculated from the core and corona contributions to the electron density:

$R_{c}^{2}($ filament $)=\left(R_{c}^{2}(\right.$ core $)+b R_{c}^{2}($ corona $\left.)\right) /(1+b)$

iii) The parallel axis theorem provides the radius of gyration of the corona with respect to the filament main axis $R_{c}^{2}$ (corona) based on the distance of the corona from the axis and $R_{G}^{2}$.

(a) $20 \mathrm{mM} \mathrm{KCl}$

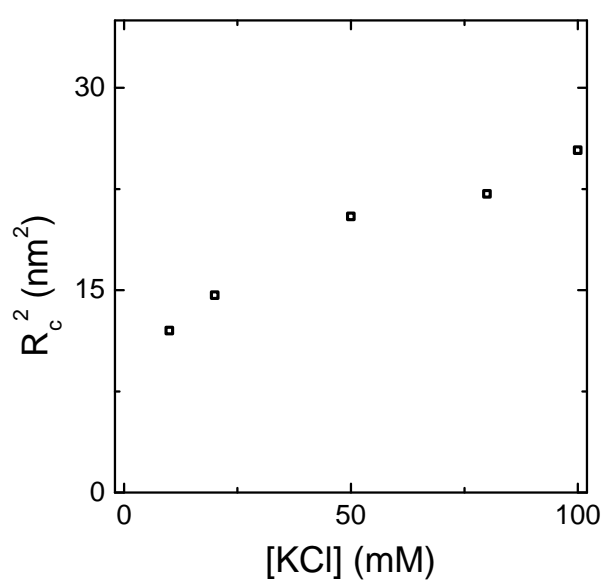

(b) $80 \mathrm{mM} \mathrm{KCl}$

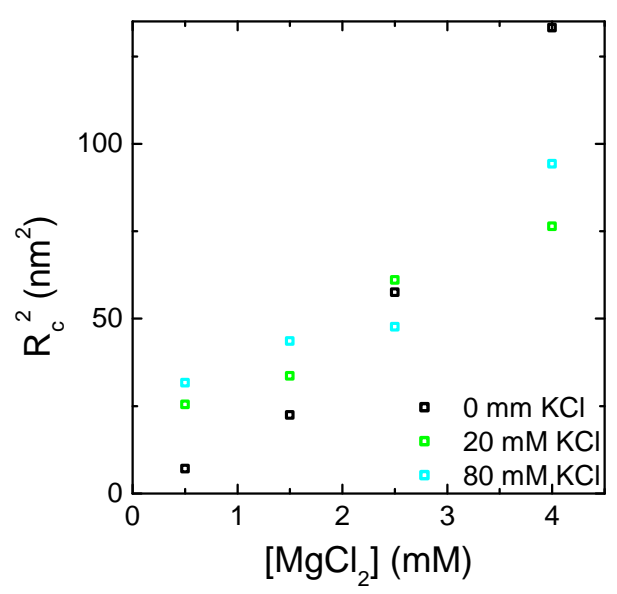

Figure 4.29: Cross-sectional radii of gyration of wild type vimentin based on equation 4.11 for (a) different potassium chloride concentrations in the absence of magnesium chloride and (b) different magnesium chloride concentrations for $0 \mathrm{mM} \mathrm{KCl}$ (black), $20 \mathrm{mM}$ $\mathrm{KCl}$ (green) and $80 \mathrm{mM} \mathrm{KCl} \mathrm{(cyan).} \mathrm{The} \mathrm{values} \mathrm{are} \mathrm{slightly} \mathrm{smaller} \mathrm{than} \mathrm{those} \mathrm{obtained}$ by the Guinier approximation (see figure 4.12).

Figure 4.29 summarizes the results for the vimentin wild type. In the presence of potassium chloride, the radii of gyration obtained by this approach tend to be up to $10 \%$ smaller than those obtained directly from the Guinier approximation. When no potassium is present, the difference is closer to $20 \%$. 


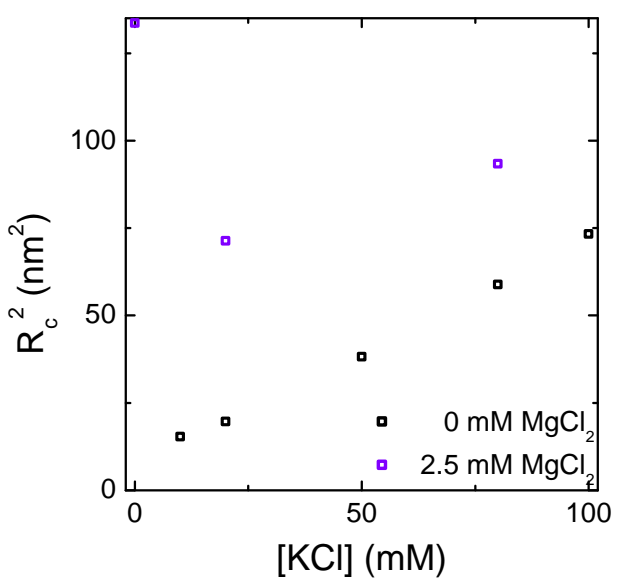

Figure 4.30: Cross-sectional radii of gyration of the $Y 11^{7} \mathrm{~L}$ mutant based on equation 4.11 at $0 \mathrm{mM} \mathrm{MgCl}_{2}$ (black) and at $2.5 \mathrm{mM} \mathrm{MgCl}_{2}$ (violet). The estimates are smaller than those from the Guinier approximation

\subsection{Interpretation}

\subsubsection{Effect of protein concentration}

The absence of changes in the scattering signal in low salt buffer for vimentin wild type protein (figure 4.2a), i.e. in conditions at which vimentin is (mostly) in its tetrameric form, is in agreement with previous findings by Sokolova et al. and Mücke et al. [30,32]: The former performed SAXS on vimentin in low salt TRIS buffer ( $\mathrm{pH} \mathrm{8.4)}$ at different protein concentrations. At $2 \mathrm{mg} / \mathrm{ml}$ and $3 \mathrm{mg} / \mathrm{ml}$ protein they reported a higher oligomeric component (32-mer) of $2 \pm 2 \%$ and only at higher concentrations of $6.6 \mathrm{mg} / \mathrm{ml}$ and $7.7 \mathrm{mg} / \mathrm{ml}$ this component increases to $22 \pm 1 \%$. In contrast, Mücke et al. determined the oligomeric composition of vi-

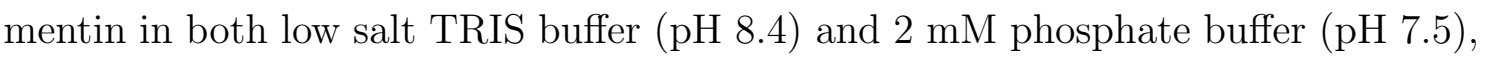
i.e. in conditions identical to those in our experiments, by analytical ultracentrifugation. For both buffer systems, they found no indication of higher oligomers between $0.4 \mathrm{mg} / \mathrm{ml}$ and $1.4 \mathrm{mg} / \mathrm{ml}$. In combination with these results, our findings therefore 
indicate that between $0.4 \mathrm{mg} / \mathrm{ml}$ and $5 \mathrm{mg} / \mathrm{ml}$ vimentin is present nearly exclusively in its tetrameric form and higher oligomers only emerge upon further increase of the concentration. For desmin, an intermediate filament from the the same class of vimentin (see table 2.1), Nicolet also observed no protein concentration dependent effect between $0.5 \mathrm{mg} / \mathrm{ml}$ and $5 \mathrm{mg} / \mathrm{ml}$ in the small angle signal at tetramer forming buffer conditions [89].

The ultracentrifugation experiments also indicate repulsive tetramer-tetramer interactions at concentrations above $0.2 \mathrm{mg} / \mathrm{ml}$. These interactions are most likely due to the negative charge of the rod domain and therefore decrease with $\mathrm{pH}$ or increasing salt concentration [43]. The small dip at $q=0.07 \mathrm{~nm}^{-1}$ in the SAXS curve for low salt conditions in figure $4.5 \mathrm{a}$ might be due to these repulsive interactions. The interactions are not visible in figure $4.2 \mathrm{a}$, as the $q$-range at these experiments is not sufficient to resolve the dip.

The addition of salt to the vimentin solution can change the dependence of the scattering signal on the protein concentration. On the one hand, the addition of salt leads to larger aggregates, effectively reducing the number density of molecules and moving intermolecular scattering to smaller $q$. On the other hand, the addition of salts can change the interaction potential which can result in a more complex behaviour. For example, $\mathrm{Y}^{3+}$ ions have been shown to turn a repulsive potential into an attractive one [90]. On the other hand, monovalent cations are known to screen electrostatic protein-protein interactions and therefore shift the onset of structure factor effects to higher protein concentrations [91]. Finally, the structure of the larger aggregates formed in the presence of cations may well depend on the protein concentration. Therefore, the lack of changes in the scattering of the vimentin wild type (figures $4.2 \mathrm{~b}$ and 4.3 ) is non-trivial.

In the presence of potassium chloride, we can compare our findings to those by Lin et al. on the effect of changes in the protein concentration between $1.5 \mathrm{mg} / \mathrm{ml}$ to $4 \mathrm{mg} / \mathrm{ml}$ on the linear elastic modulus of vimentin filaments [38]. Their results are in good agreement with models for solutions of entangled, semi-flexible polymers which assume no change in the properties of a single filament [92, 93].

When regarding the effect of the protein concentration on the scattering in solutions containing both potassium and magnesium chloride, the comparison with rheology experiments becomes more complex: In experiments on the cross-linking properties of ions, it is common to keep the ratio between cross-linkers and filaments fixed when investigating the influence of protein concentration, i.e. an increase in protein concentration is accompanied by an increase in the number of cross-linking ions, to 
keep the number of cross-linkers per filament fixed. The observed increase of elastic modulus with increasing protein concentration at fixed magnesium to protein ratio can be explained with a model which keeps filament properties constant, which our findings do not contradict [38, 39, 94].

In the presence of only magnesium chloride, we observe a systematic change in the structures formed above $4 \mathrm{mg} / \mathrm{ml}$ protein, see section 4.2 : Whereas the samples appear homogeneous to the blank eye below that concentration and their scattering curves match each other, at $5 \mathrm{mg} / \mathrm{ml}$ the sample becomes heterogeneous on a millimeter scale, indicating the formation of very large aggregates or some kind of phase separation.

In contrast to the above, the scattering signal of the $Y 11^{7} \mathrm{~L}$ mutant in the presence of potassium chloride does show a protein concentration dependence: The curves become more similar to the scattering curves of the wild type at higher concentrations. Also, the increase of the slope towards low $q$ indicates longer aggregates. This could be due to incomplete suppression of the elongation which becomes more likely with increasing protein concentration and hence increased ULF end-to-end contacts. We therefore cannot exclude the presence of filaments in our data on $Y 117 \mathrm{~L}$.

\subsubsection{Effect of changes in the ionic compositions}

The SAXS curves of vimentin in different ionic environments offer a wealth of information on its structure at these conditions. In the following, we will discuss the consequences of our findings and compare them to the literature.

There are some basic conclusions that can be drawn directly from the curves, without any need for further interpretation:

- The structure of vimentin is sensitive to the ionic species and their respective concentrations in the buffer

- Monovalent and divalent cations induce systematically different structures

- Higher monovalent ion concentrations reduce the effect of divalent ions on the structure

The fact that filaments formed in the presence of both monovalent and divalent ions differ from those formed in the abscence of divalent ions contrasts the finding by Lin et al. who found no change in filament morphology by TEM between $0 \mathrm{mM}$ and 
$4 \mathrm{mM} \mathrm{MgCl}_{2}$ [38]. They also could explain rheological data up to $16 \mathrm{mM} \mathrm{MgCl}_{2}$ with magnesium as a cross-linker, which does not affect the structure of individual filaments. However, their experiments were performed at $160 \mathrm{mM} \mathrm{NaCl}$ and we also see that increasing the monovalent ion concentration reduces the effect of magnesium on the structure. It is therefore well possible, that any structural change at $160 \mathrm{mM} \mathrm{NaCl}$ is not detectable by TEM and also mechanical properties change only below the detection level of rheology. For other intermediate filament species differences between filaments formed in the presence of monovalent ions only and in the presence of both mono- and divalent ions have been reported: For desmin, Stromer et al. reported similar filament diameters but different absorbance at $300 \mathrm{~nm}$ [42]. For keratin K8/K18 IFs recent results show that adding $1 \mathrm{mM} \mathrm{MgCl}$ to the assembly systems increases the persistence length of the resulting filaments from about $300 \mathrm{~nm}$ to about $480 \mathrm{~nm}$ [95].

\section{Tetrameric vimentin}

When discussing the scattering and structure of tetrameric vimentin, it is important to remember that "low salt" and no magnesium or potassium do not imply the abscence of any ion. Indeed besides the phosphate anions, the buffer also contains about $2.5 \mathrm{mM}$ monovalent sodium cations. When compared to the lowest magnesium concentration in our experiments - $0.5 \mathrm{mM}$ - this might actually be a non-negligible amount.

We can compare the PDDFs we obtained for the tetrameric state of both wild type and the $Y 117 L$ mutant (figures $4.14 \mathrm{a}$ and 4.16) to those obtained by Sokolova for a different point mutation $(K 139 C)$ [30]. We see that all three curves share the main features: A peak at $1.5 \mathrm{~nm}$ and a shoulder at about $4 \mathrm{~nm}$, indicating that all three types of vimentin have identical tetrameric structures. The fact that Sokolova et al. could model the SAXS signal, including both the peak and the shoulder in the PDDF with a vimentin tetramer indicates that these structural features of the PDDF stem only from tetrameric vimentin. The fact that the peak at $1.5 \mathrm{~nm}$ remains the only peak besides the main peak at higher salt concentrations indicates the abscence of octamers or hexadecamers in our samples. Interestingly, for the intermediate filament desmin, Nicolet reported a PDDF which shares the same main features (peak and shoulder) but both are shifted to slightly larger distances [89]. Our estimates for the cross-sectional size of the tetramer - a cross-sectional radius of gyration of $R_{c}^{2}=5.25 \mathrm{~nm}^{2}$ and in the core-corona model a central radius of $2.3 \mathrm{~nm}$ 
and a gyration radius of the side chains of $3.4 \mathrm{~nm}$ - are in good agreement with previous results: From [34] the diameter of the tetramer can be estimated to about $5 \mathrm{~nm}$. A lower border for the size of the corona can be given using the number of amino acids in the head and tail and the persistence length per amino acid in a peptide loop (about $0.3 \mathrm{~nm}$ ) to equal $1.7 \mathrm{~nm}$ [96]. When we consider that the side chains possess more than one negative charge [38] and that the $2.5 \mathrm{mM} \mathrm{Na}^{+}$ ions present in the low salt buffer result in an electrostatic screening length of about $6 \mathrm{~nm}$ [54], a higher radius of gyration is actually expected.

In addition to obtaining structural results on the tetramer, its SAXS data provides us with two ways to estimate the amount of vimentin remaining in the tetrameric state for different salt concentrations: The parameter $t$ from the model-based analysis which decribes how much vimentin remains tetrameric and the height of the remaining peak at $1.5 \mathrm{~nm}$ in the PDDFs. For both the $Y 117 \mathrm{~L}$ mutant and the wild type, at $10 \mathrm{mM} \mathrm{KCl}$ both the PDDFs and the value of $t$ indicate nearly as much vimentin in tetrameric form as in filaments. Further increase of the potassium concentration reduces the tetrameric component. At $50 \mathrm{mM} \mathrm{KCl}$, interestingly, both a shoulder in the PDDF and the value of $t$ indicate that the tetrameric component for the $Y 177 \mathrm{~L}$ mutant is significantly larger than for the wild type. In the presence of $0.5 \mathrm{mM} \mathrm{MgCl}_{2}$ the model-based approach also returns a high proportion of vimentin in tetrameric form. However, whereas the PDDF at $1.5 \mathrm{~nm}$ is rather high, no clear shoulder or peak can be distinguished. This may imply that the present structure is actually only very closely resembling the tetramer, but not necessarily is a tetramer. Finally, we need to note that the Guinier-approximation cannot distinguish between contributions from tetramers and such from filaments. Therefore, especially at lower salt concentrations, the radii of gyration of the filaments in the solution are actually larger than the values given in figures 4.12 and 4.13, as those are the weighted means of the squared radii of gyration of tetramers and filaments.

\section{Structure of vimentin filaments}

Next, we discuss what we can learn about the structure of vimentin filaments and its dependence on the ionic compositions of the buffer from the SAXS data. The first important result is that the SAXS signal of vimentin (and hence its structure) shows a dependence of a monovalent cation concentration. This actually distinguishes vimentin from desmin, which (in "TRIS" buffer) reportedly shows no changes in the scattering signal. Additionally, the structure in the PDDFs differs as for desmin the peak at about $1.5 \mathrm{~nm}$ persists as the main peak. However, some desmin mutants, 
which have been confirmed by TEM to assemble to seemingly normal filaments, have PDDFs and salt concentration dependent changes of the PDDF which are very similar to what we observe for vimentin [89].

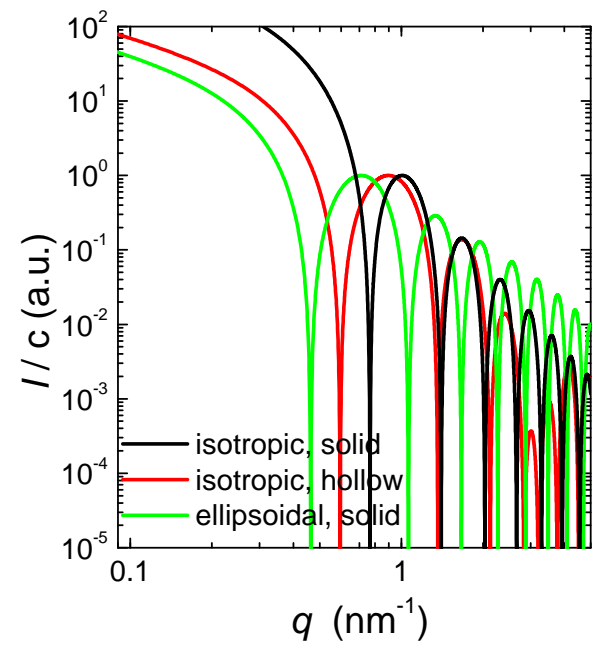

Figure 4.31: Comparisons of formfactors of long rods with different cross-section geometries, scaled such that their first local maxima are of equal height: a solid, isotropic crosssection with a radius of $5 \mathrm{~nm}$ (black), a hollow, isotropic cross-section with an inner radius of $3 \mathrm{~nm}$ and an outer radius of $5 \mathrm{~nm}$ (red), and a solid, ellipsoidal cross-section with a longer half-axis of $5 \mathrm{~nm}$ and a shorter half-axis of $3.75 \mathrm{~nm}$ (green).
One open question in the research of intermediate filament structure is whether individual filaments are solid or hollow and whether their cross-section is isotropic or ellipsoidal [26, 30, 97]. We were able to describe the filaments with solid, isotropic central cores, but we also saw that the ratio between the height of the first and second local maximum present at $80 \mathrm{mM}$ and $100 \mathrm{mM}$ is slightly overestimated by this model. As the maxima in these cases are mostly due to the central core scattering we can compare the form factors for a solid, isotropic cross-section, a hollow, isotropic cross-section and a solid, ellipsoidal cross-section to see how these changes in geometry alter the height ratio. Figure 4.31 shows such a comparison scaled such that their first local maxima are of equal height for easier comparison of the ratio of the height of

the first and second maxima. We see that there is next to no difference in the height of the maxima for the two isotropic cases. When we compare the ellipsoidal crosssection to the isotropic cross-section, we see that the ratio in height decreases, i.e. the second maximum is higher. This implies some ellipsoidity in the cross-section, as Sokolova et al. suggested for the ULF in [30]. Also, an ellipsoidal core leads to larger cross-sectional radii of gyration and hence might be the cause for the underestimate noted in section 4.4.2. However, as we can see from figure 4.20a, the difference between the two models is less than the noise level, and therefore we can neither confirm nor exclude an ellipsoidal cross-section.

One of the most persistent effects of increasing salt concentration, both for monoand di-valent ions, is an increase in filament size: With increasing salt concentration 
the slope in the low- $q$ region of the scattering curves becomes steeper, the radius of gyration grows, the peaks of the PDDFs shift to larger values and the fits to form-factor return a higher optimal radius $R$ of the core. When we compare the peak position of the PDDF to $R$, the peaks provide a lower estimate of the mean size of the filaments for low salt concentrations (e.g. $3.5 \mathrm{~nm}$ as opposed to $4.5 \mathrm{~nm}$ from the fit at $10 \mathrm{mM} \mathrm{KCl}$ ). This can however be explained by the fact that in the PDDF we see an overlap between the tetramers and the filaments which have been separated in the model-based fits. But how do the filament diameters we obtain compare to previous results?

Mücke at al. previously reported a filament diameter of $9.4 \mathrm{~nm} \pm 2.7 \mathrm{~nm}$ in "phosphate" buffer at $100 \mathrm{mM} \mathrm{KCl}$ by TEM [32]. For human vimentin, it is known that the mass per length at $2.5 \mathrm{mM} \mathrm{MgCl}_{2}$ in a weaker "phosphate" buffer (0.7 mM phosphate) forms a bi-gaussian distribution with peaks at $71 \mathrm{kDa} / \mathrm{nm}$ and $106 \mathrm{kDa} / \mathrm{nm}$ whereas in "TRIS" buffer at $50 \mathrm{mM} \mathrm{NaCl}$ the mass per length has peaks at $38 \mathrm{kDa} / \mathrm{nm}, 47 \mathrm{kDa} / \mathrm{nm}$ and $56 \mathrm{kDa} / \mathrm{nm}$, i.e. about a factor two lower. In electron micrographs, the diameter at $2.5 \mathrm{mM} \mathrm{MgCl}_{2}$ also appears larger (about $15 \mathrm{~nm}$ to $20 \mathrm{~nm}$ based on the figures), but no value is given [23]. For different vimentin species at $5 \mathrm{mM} \mathrm{CaCl}_{2}$ in "TRIS" buffer, the diameters of filaments are known to be larger than those in the presence of $160 \mathrm{mM} \mathrm{NaCl}: 15.9 \mathrm{~nm} \pm 2.2 \mathrm{~nm}$ to $11.6 \mathrm{~nm} \pm 0.9 \mathrm{~nm}$ for bovine vimentin and $24.2 \mathrm{~nm} \pm 8.5 \mathrm{~nm}$ to $12.2 \mathrm{~nm} \pm 1.2 \mathrm{~nm}$ for recombinant Xenopus vimentin [36], but it is unclear how these results can be transferred to human vimentin in the presence of magnesium chloride.

For $100 \mathrm{mM} \mathrm{KCl}$, we find a squared cross-sectional radius of gyration of about $28 \mathrm{~nm}^{2}$, a peak in the PDDF at about $5.4 \mathrm{~nm}$, and in the model-based fit a mean core radius of $6.3 \mathrm{~nm}$ and a radius of gyration of the corona of $3.5 \mathrm{~nm}$ (resulting in a squared cross-sectional radius of gyration of about $25 \mathrm{~nm}^{2}$ ). In addition, the position of the local minima (table 4.1) corresponds to a cylinder radius of about $6 \mathrm{~nm}$. This means the diameter of the filaments determined by SAXS is larger than the one determined by TEM at identical buffer conditions. There are several possible reasons for this:

- different assembly times and temperatures: samples for TEM were assembled for $1 \mathrm{~h}$ at $37^{\circ} \mathrm{C}$, as compared for SAXS samples which were assembled for over $24 \mathrm{~h}$ at $4{ }^{\circ} \mathrm{C}$ (and did not change their signal during the up to over $20 \mathrm{~h}$ in the experimental set-up at room temperature). Also, typical protein concentrations for the TEM studies were about an order of magnitude smaller.

- different sample environments: SAXS samples were in solution whereas TEM 
samples were fixed and stained on EM grids. We can not exclude that these steps affect the filament diameter.

For wild type vimentin at $2.5 \mathrm{mM} \mathrm{MgCl}_{2}$ we estimate the squared cross-sectional radius of gyration to $66 \mathrm{~nm}^{2}$, the peak of the PDDF lies at about $7.8 \mathrm{~nm}$ and the fit to the core-corona model returns $R=8.4 \mathrm{~nm}$ and $R_{G}=6.5 \mathrm{~nm}\left(R_{C}^{2}=58 \mathrm{~nm}^{2}\right)$. This is comparable to the results by TEM at identical salt concentration.

The dependence of filament properties on the salt concentration has been studied mostly by viscosimetry and rheology. In the presence of a single ion species (sodium, potassium, magnesium or calcium) different studies showed that the bulk viscosity increases with the salt concentration $[32,36]$. When we combine this with our insight that the diameter of the filaments increases the higher viscosity might be due to an increase in filament persistence length which in turn would be due to the increased diameter [98-101]. Hence, the general trends appear to agree though no quantitative statements can be made.

The forward scattering amplitudes obtained from the Guinier approximation provide us with an additional approach to compare our results to previous experiments, as

$$
I_{0}=\lim _{q \rightarrow 0, c \rightarrow 0}(q I(q)) \propto(\Delta \rho)^{2} M_{L}
$$

where $\Delta \rho^{2}$ is the electron density contrast and $M_{L}$ the mass per length $[102,103]$. Therefore, for constant contrast and concentrations the forward scattering amplitudes are proportional to the mass-per-length. When we compare the forward scattering in the presence of $100 \mathrm{mM} \mathrm{KCl}$ to that in the presence of $2.5 \mathrm{mM} \mathrm{MgCl}_{2}$ we get

$$
\frac{I_{0}\left(2.5 \mathrm{mM} \mathrm{MgCl}_{2}\right)}{I_{0}(100 \mathrm{mM} \mathrm{KCl})} \approx \frac{61.9}{28.4} \approx 2.2
$$

This is in the same range as the ratio in mass per length reported by Herrmann et al., which is also in the order of two (see above).

It is actually non-trivial that the electron density is identical for different filament structures even if all of them are built from vimentin tetramers, as the spacing between individual tetramers is not necessarily constant. Therefore, it is of interest to investigate how the forward scattering from the central rod depends on the radius of the central rod $R$. When we consider the pre-factors of the form-factor for cylinders (e.g. appendix B.3), we see that $\gamma \propto(\Delta \rho(R))^{2} R^{4}$, i.e. for a constant electron density $\gamma$ would grow as $R^{4}$ and for a fixed number of electrons per length it would remain constant. It should be noted that attempts to model $\gamma \propto R^{4}$ to account for 
changes in the electron density due to radial polydispersity yielded no satisfactory fits (data not shown).

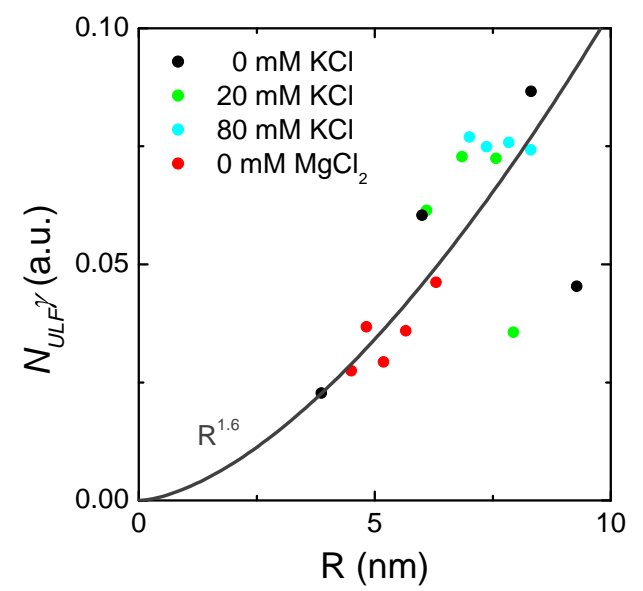

Figure 4.32: The forward scattering of the central rod $\left(N_{U L F} \gamma\right)$ versus the core radius $R$ for different salt concentrations. The two points on the right hand side are recorded at $4 \mathrm{mM} \mathrm{MgCl}_{2}$. $\gamma$ is proportional to $(\Delta \rho(R))^{2} R^{4}$.
In figure 4.32 we see that in general $\gamma$ increases with $R$. The only major outliers are results from $4 \mathrm{mM} \mathrm{MgCl}_{2}$ at $0 \mathrm{mM}$ and $20 \mathrm{mM} \mathrm{KCl}$ respectively. When we neglect these outliers, the remaining data points can be fitted to a power law which yields that $\gamma$ goes as $R$ to the power of $1.6 \pm 0.2$, resulting in $\Delta \rho \propto R^{-1.2}$, or in terms of the massper-length $M_{L} \propto R^{0.8}$. This implies that with increasing radius (which we saw to be equivalent to increasing salt concentration), the packing of vimentin tetramers in the filament decreases. One possible interpretation of this behaviour is that the filaments are indeed hollow, as mentioned above.

In contrast, the inverse number density of Gaussian side chains $\lambda$ does not follow a common scaling law, although for a fixed potassium concentration it decays strongly with the radius, with a power larger than 4 (see figure 4.28). Assuming that $\lambda$ is inversely proportional to the number of monomers in the cross-section, this would give $\Delta \rho \propto R^{2}$, i.e. a density which grows with the radius. One possible explanation for this behaviour is that the relation between $\lambda$ and the number of monomers might be more complex than expected.

Finally we can consider the size of $b$, the parameter describing the ratio of scattering from the corona to scattering from the rod, and its changes upon salt concentration changes. In the abscence of magnesium chloride, $b$ is in the order of 0.3. Assuming one side-chain per vimentin monomer, $b$ should correspond to the ratio of excess scattering mass of vimentin in the tail domain to that in the rod and head domain. When we estimate this by the numbers of amino acids we obtain $b_{A A}=\frac{55}{77+334}=0.133$ which is nearly a factor three smaller than the observed value. However, as we kept $\lambda$ fixed when determining $b$ and the fit is actually sensitive to $\lambda b^{2}$ (see figure 4.20a) this is only a very rough estimate which nevertheless does correctly reproduce the right order of magnitude. So far, we saw that changes in the potassium chloride con- 
centration in the abscence of magnesium chloride do not systematically change $b$. In contrast, adding magnesium chloride does increase $b$ up to above 2 at $4 \mathrm{mM} \mathrm{MgCl}_{2}$. Assuming that the number of amino acids in the tail remains constant, there must be some other mechanism to increase the electron density and thereby the scattering length in the corona. One possibility is the condensation of magnesium ions in the originally negatively charged corona, i.e. counter-ion condensation $[38,104]$. As magnesium has more electrons than typical atoms in proteins, its excess scattering length is higher, and therefore a locally increased magnesium concentration in the corona would result in an increase in $b$. Therefore the increase of the scattering from the corona is a distinct hint towards counter-ion condensation of magnesium.

The last structural factor we consider is the radius of gyration of the corona, $R_{G}$. Like $b$, it shows no trend for increasing potassium chloride concentration, but increases with increasing magnesium concentration. Again, counter-ion condensation offers a plausible explanation: The localization of divalent ions at the negatively charged amino acids of the tail results in a charge inversion whose strength increases with increasing (local) magnesium concentration [38,105]. This in turn increases repulsion within the side chain and increases its radius of gyration.

The increased local magnesium concentration might also be responsible for the extended tail towards longer distances in the PDDFs: The distance of magnesium ions in the condensation shell can well exceed any distance found in the vimentin filament itself and would result in a significant contribution at larger distances.

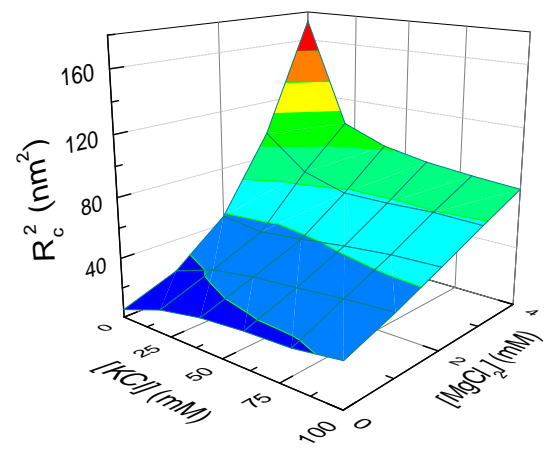

Figure 4.33: Dependence of the crosssectional radius of gyration on the potassium and magnesium concentration.

of these effects with increasing potassium chloride concentration becomes quite log-
Figure 4.33 illustrates our finding that in solutions containing either magnesium and potassium ions the filament diameter increases with the cation concentration but in solutions containing both ions the correlation between ion concentration and filament diameter is more complex: At higher potassium concentrations, the effect of the magnesium ions reduces. When we think about the effects of increasing magnesium chloride concentration in terms of counterion condensation, the reduction 
ical: At higher potassium chloride concentrations the screening of electrostatic interactions, which are the basis for counterion condensation, increases and hence counterion condensation is reduced [104]. We will further discuss vimentin as a polyelectrolyte and the implications of our findings in chapter 8 .

\section{Changes in the structure of the $Y 117 L$ mutant}

Finally, we can discuss the change induced by different ionic compositions in the assembly state of the $Y 117 \mathrm{~L}$ mutant. As we discussed above (4.5.1), the structure of $Y 11^{7} \mathrm{~L}$ depends on the protein concentration. As all previously reported results were recorded at about a tenth of the concentration we used in our experiments, comparisons to them are not feasible [43]. We will therefore only compare the mutant to the wild type.

The first remarkable fact is that at $10 \mathrm{mM} \mathrm{KCl}$, the scattering curves for the mutant and for the wild type are nearly identical and major differences only emerge at higher salt concentrations, where the SAXS curves from the mutant protein do not display the features found for the wild type protein (local maxima, kinks). Nevertheless, the curves for the wild type protein can be described by the same model as those for the mutant protein, indicating some structural similarities.

One of the most consistent differences between the mutant and the wild type at identical ionic environments is that the mutant appears to form aggregates with a larger diameter: In the SAXS curves both their forward scattering amplitude and the steepness at low- $q$ are larger, which is directly reflected by the Guinier approximation. The comparison of the cross-sectional radii of gyration and the forward scattering amplitudes also shows that both values continue to steadily increase with increasing potassium concentration for the mutant protein, whereas they appear to level off for the wild type protein. In the PDDFs the peaks are always located at larger distances, for example at $7.5 \mathrm{~nm}$ for the mutant protein as opposed to $5.3 \mathrm{~nm}$ for the wild type protein at $100 \mathrm{mM} \mathrm{KCl}$. When we compare the fit parameters, we see that not only the radii appear larger but also the radii of gyration of the corona. For higher potassium concentrations, the forward scattering amplitude of the core $N_{U L F} \gamma$ of the mutant protein is smaller, whereas $b$ rises steadily with the potassium concentration. The larger contribution of scattering from the side chains is most likely responsible for the reduced amount of structure on the SAXS curves as the structural elements from the different contributing components can cancel each other out. As for the wild type protein in the presence of magnesium, the 
increased scattering from the corona is most likely responsible for the extended tails observed in the PDDFs. The increased scattering and apparent size of the side chains in comparsion to the wild type at identical salt conditions might be due to a re-arrangement of the vimentin tail regions during the compaction to mature filaments.

The fact that the SAXS curves of the mutant protein can be modeled with the same polydispersity model as those for the wild type protein implies that its structures actually are well defined and not random aggregates. There a two different possibilities to explain the observed increase in filament diameter: Either more vimentin monomers contribute to the cross-section or the cross-section does not compact (see section 2.1.2). Several results from fitting to the model hint towards the second scenario: Whereas the radius of the solid core $R$ remains in the same size range as for the wild type, the radius of gyration of the corona increases more strongly. Additionally $(\Delta \rho)^{2} \propto \gamma R^{-4}$ is about a factor of two smaller and $b$ is considerably larger (see figures 4.22 to 4.27 ), indicating a decrease of mass in the core and an increase of mass at the edge of the filament. Finally, $\lambda$ remains nearly constant, which also indicates a constant number of monomers in the cross-section. The value of around $2 \mathrm{~nm}$ which the fits return for $\lambda$ would actually be consistent with a loose, non-overlapping alignment of ULFs as 32 tails on a $60 \mathrm{~nm}$ long ULF.

Based on TEM data, Y117L is also reported to have a salt concentration dependent diameter, varying from $11 \mathrm{~nm}$ at $30 \mathrm{mM} \mathrm{KCl}$ to $13 \mathrm{~nm}$ at $100 \mathrm{mM}$ and $22 \mathrm{~nm}$ at $160 \mathrm{mM}$ [43]. This compares surprisingly well to our results for the rod diameter: about $10.5 \mathrm{~nm}$ at $20 \mathrm{mM} \mathrm{KCl}$ and $14 \mathrm{mM}$ at $100 \mathrm{mM} \mathrm{KCl}$. To summarize, it seems that at about $3 \mathrm{mg} / \mathrm{ml}$ protein, potassium chloride leads to lateral and at least to some extent also longitudinal annealing of $Y 117 \mathrm{~L}$ filaments but the final compaction of filaments observed for the wild type does not seem to take place. 


\section{Chapter 5}

\section{Microfluidics for SAXS}

So far, we have discussed how different ionic environments affect the equilibrium state of vimentin. But how do self-assembly kinetics and intermediate states, like ULFs, depend on these conditions? This chapter explains how microfluidics can be used to access these questions. Towards this goal, it introduces basic concepts of microflow experiments. The special requirements which the combination with SAXS experiments pose on microfluidic devices are discussed and the fabrication process of the devices used for this work is presented. Finally, the basic concepts of letting vimentin assemble in these devices is presented. Parts of this chapter have already in published in Lab on a Chip [106].

\subsection{Flow at low Reynolds numbers}

Microfluidics refers to the manipulation of small volumes of fluids - flows in the range of $\mu \mathrm{l} / \mathrm{h}$ or less in channels in the sub-mm range. This offers the obvious advantage of requiring little amounts of sample but also decreased reaction and hence analysis times.

Applications of microfluidics vary over a broad range: In structural biology, microfluidics are applied for automatic screening for crystallization conditions [107], in fiber synthesis controlled mixing of reactants can improve product quality [108], in polymer science confinement of single polymers allows control of models for polymers $[11,109]$ and encapsulation of single cells offers microreactors for single-cell experiments [110]. 


\subsubsection{Viscous flow}

As any flow of an incompressible fluid with constant viscosity, microflow follows the Navier-Stokes equation

$$
\rho\left(\frac{\partial \vec{v}}{\partial t}+(\vec{v} \nabla) \vec{v}\right)=-\nabla p+\eta \Delta \vec{v}+\rho \vec{f}
$$

where $\rho$ is the density of the fluid, $\vec{v}$ the velocity field, $p$ the pressure, $\eta$ the dynamic viscosity and $\vec{f}$ an externally applied bulk force, e.g. gravity. We can introduce dimensionless variables for space $\vec{x}^{\prime}=\vec{x} / L_{F}$ and velocity $\vec{v}^{\prime}=\vec{v} / U$ where $L_{F}$ is the typical length scale of the system and $U$ the typical velocity. In direct consequence we obtain $p^{\prime}=p L_{F} / U \eta$ and $t^{\prime}=t U / L_{F}$. In the abscence of external forces we can then rewrite 5.1

$$
\frac{\rho U L_{F}}{\eta}\left(\frac{\partial \vec{v}^{\prime}}{\partial t^{\prime}}+\left(\vec{v}^{\prime} \nabla^{\prime}\right) \vec{v}^{\prime}\right) \equiv \operatorname{Re}\left(\frac{\partial \vec{v}^{\prime}}{\partial t^{\prime}}+\left(\vec{v}^{\prime}\left(\nabla^{\prime}\right) \vec{v}^{\prime}\right)\right)=-\nabla^{\prime} p^{\prime}+\Delta^{\prime} \vec{v}^{\prime}
$$

introducing the dimensionless Reynolds number $\operatorname{Re}=\frac{\rho U L_{F}}{\eta}$ which corresponds to the ratio of inertial forces to viscous forces in the flow. Two flows in similar geometries are considered similar when their Reynolds number is equivalent. For water $\frac{\rho}{\eta}=10^{4} \mathrm{~mm}^{-2}$ which for $U \approx 1 \mathrm{~mm} / \mathrm{s}$ and $L \approx 0.1 \mathrm{~mm}$ results in $\operatorname{Re} \approx 0.1$. This is much lower than the Reynolds number under which turbulence typically sets in, $\mathrm{Re} \approx 2000$ [111]. Therefore, microfluidic flow is laminar and consequently exactly controllable. Additionally for small Reynolds numbers $\operatorname{Re}\left(\frac{\partial \vec{v}^{\prime}}{\partial t^{\prime}} \vec{v}^{\prime} \nabla^{\prime}\right) \approx 0$ and equation 5.1 simplifies to

$$
\eta \Delta \vec{v}=\nabla p
$$

i.e. the flow becomes time-reversible.

In addition to the Navier-Stokes equation conservation of mass results in the continuity equation

$$
\frac{\partial \rho}{\partial t}+\nabla(\rho \vec{v})=0
$$

which for stationary, incompressible flow implies that the flow is divergence free, i.e.

$$
\nabla \vec{v}=0
$$




\subsubsection{Distribution of solutes in flow}

The distribution of a solute in a flow system is governed by two processes: convection with the flow and isotropic diffusion.

The convectional flux of a solute is simply the product of its concentration $c$ with the flow field

$$
\vec{j}_{c o}=c \vec{v}
$$

The typical time a solute requires to pass a distance $L_{T}$ by convection scales as $t_{c o}=L_{T} / V$ where $V$ is the typical velocity of the flow. Diffusion follows Fick's first law correlating solute flux and the concentration gradient

$$
\vec{j}_{d i}=-D \nabla c
$$

where $D$ is the diffusion constant. In contrast to convection, the typical time a solute requires to pass a distance $L_{T}$ by diffusion scales with the square of the distance: $t_{d i}=L_{T}^{2} / D$. Inserting equations 5.6 and 5.7 into the continuity equation 5.4 , with the solute concentration corresponding to the density, results in the convectiondiffusion equation

$$
\frac{\partial c}{\partial t}+\nabla(c \vec{v})=D \Delta c
$$

As for the flow itself, there is a dimensionless number for the transport of solutes, the Péclet number

$$
\mathrm{Pe}=\frac{t_{d i}}{t_{c o}}=\frac{L_{T} U}{D}
$$

For small Péclet numbers, the transport is dominated by diffusion. In the limit of $\mathrm{Pe} \rightarrow 0$ the solute would fill a microfluidic device homogeneously in equilibrium. In the other extreme the solute strictly follows the flow lines. Hence, by altering the Péclet number by varying either flow speeds or typical length scales it is possible to control the distribution of a solute in a microfluidic device, for example to create gradients or induce mixing. For proteins with diffusion constants in the range of $10^{-11} \mathrm{~m}^{2} / \mathrm{s}$ at $U \approx 1 \mathrm{~mm} / \mathrm{s}$ and $L_{T} \approx 0.1 \mathrm{~mm}$ the Péclet number is $10^{4}$ and for small ions with diffusion constants in the range of $10^{-9} \mathrm{~m}^{2} / \mathrm{s}$ it is only 100 . We see that for proteins, transport in microfluidic flow is strongly convectional whereas for ions the contribution of diffusion is significant. 


\subsection{Microfluidic mixers}

As discussed in section 2.1.1 mixing of monovalent ions with vimentin tetramers initiates the assembly of vimentin filaments. But how can we achieve controlled, stationary mixing of these two components in a microfluidic device without applying external forces such as electromagnetic forces?

Due to the laminarity of microfluidic flow, mixing becomes a purely diffusional process. Mixing times are therefore in first approximation equivalent to diffusion times. For small ions on microfluidic length scales these are in the range of seconds as discussed in section 5.1.2. This low mixing efficiency is adventageous when intending to create stable concentration gradients [112]. However when the goal is complete mixing, the low efficiency may not be sufficient. For many applications it is therefore necessary to improve the flow geometry to enhance mixing. This can be achieved by decreasing the typical distance solutes have to cross for mixing and by increasing the surface area.

One possible approach is to create several alternating layers of the solutions to be mixed [113], which can be extended to several layer splitting and recombination steps increasing the efficiency by a factor of more than 10 [114]. However, these approaches rely on complex flow geometry and changing the mixing conditions requires changes in the channel layout.

For the initiation of vimentin assembly, where small ions need to be added to weakly diffusive proteins, we can therefore apply a more basic approach as sketched in figure 5.1. The length required for mixing is reduced by hydrodynamic focusing of the protein solution from both sides with salt buffer in a cross-shaped device. As the protein diffusivity is low it forms a jet in the middle of the device into which the ions can diffuse and initiate the assembly process. Adjusting the relative flow rates of protein and salt solutions, respectively, tunes the width of the protein jet and hence controls mixing [115]. The very simple layout in combination with the possibility to control mixing via the flow rates render hydrodynamic focusing a suitable approach for studying systems in which a small reactant needs to be added to a weakly diffusive reactant: The self-assembly of collagen in a $\mathrm{pH}$ gradient [116], controlled spinning of silk fibers [108,117], dynamics of the compaction of DNA by polypropyleneimine dotriacontaamine dendrimers [118], protein folding upon decrease of denaturant concentration [60] or RNA folding induced by the addition of magnesium ions [119] have been investigated with this kind of mixers. 


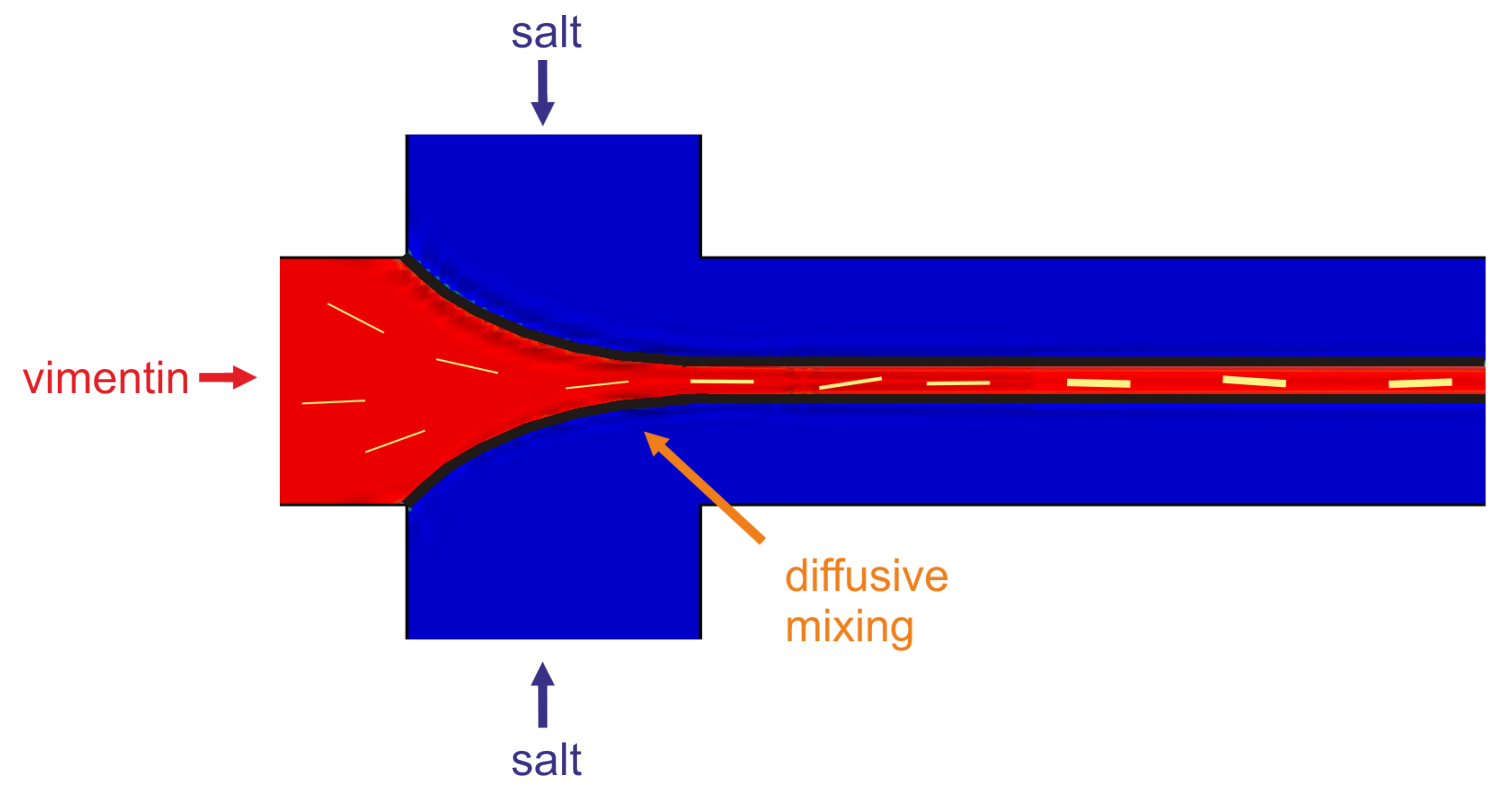

Figure 5.1: Schematic of the microfluidic layout used for studying the vimentin assembly. Vimentin solution enters the device from the central inlet, salt buffer from the sides. The buffer hydrodynamically focuses the vimentin solution to a jet. As it flows downstream, the salt diffuses into the vimentin jet and initiates the assembly process. By probing different locations of the jet different time points of the assembly and different salt concentrations can be probed. Variation of absolute and relative flow speeds allows for different combinations of reaction times and salt concentrations.

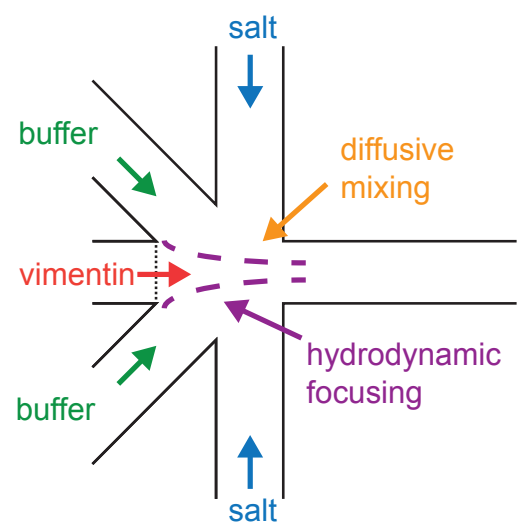

Figure 5.2: Sketch of a mixer which employs pre-focusing to reduce the diffusion time. A thin layer of sample buffer at low flow speed prefocuses the sample solution such that the width of the jet the salt needs to diffuse into is reduced, effectively lowering the time-scale for diffusion.
In principle, the time resolution of a hydrodynamic focusing mixer can be improved by adding an additional thin fluid layer (e.g. sample buffer) between the protein solution and the salt buffer, as illustrated in fig. 5.2. This layer is injected into the device in a $45^{\circ}$ angle at the cross-section and poses an initial barrier to diffusion of salt ions into the protein solution. Thereby it separates the hydrodynamic focusing and the mixing and postpones the beginning of mixing to an already focused protein jet, reducing the length the ions need to diffuse. As the diffusion time depends on the square of the distance (see 5.1.2) this effectively reduces the mixing time while maintaining the adjustability of the hydrodynamic focusing mixer. The flow rate of the additional layer is somewhat critical as a too thin layer results in premature mixing and a too thick 
layer poses an unnecessary barrier. A flow rate of about $1 \%$ to $5 \%$ of the total flow in the mixers appears to be optimal [120].

\subsection{Avoiding surface effects}

When intending to study reactions in microflow, interactions of the solutes with the surface of the channel can result in a number of undesired effects [121], for example

- leakage of solutes into the device material, reducing actual concentrations

- absorbance of solvent by the device

- dissolution of device material into the fluid, altering chemical properties

- adhesion of reactants to the channel walls, preventing the formation of a stable flow.

Besides choosing a material inert to all reactants, avoiding contact between the surface and the reactants ensures the abscence of such surface effects. For the vimentin assembly, filaments and intermediates adhere to the channel walls when no countermeasures are taken. Several solutions to this problem have been proposed [10,122], for example enclosing the protein in drops [10] or coating of the channel walls [123]. Simulations show that in principle it is even possible to design nano-stuctured surfaces that repel soluted macromolecules [124].

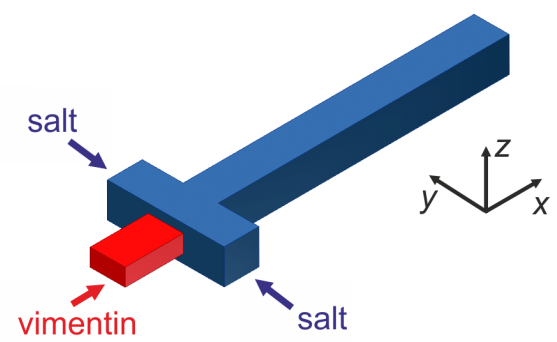

Figure 5.3: Sketch of the microfluidic channel geometry. The central inlet is shallower than the side inlets and the outlet, preventing contact between assembling vimentin and the channel walls.
Another approach, that is well suited for weakly diffusive proteins, is a variation of the hydrodynamic focusing mixer described above (section 5.2). By decreasing the height of the protein inlet channel (see figure 5.3) to be smaller than all other inlets and the outlet, the buffer solution from the side channels can engulf the flow of the protein solution and form a layer between the protein and the channel walls not only from the sides but also from the top and bottom, thereby preventing any contact between protein and the channel walls as soon as mixing starts $[108,119]$. When the Péclet number is sufficiently high, i.e. 
layer thickness and flow velocity are large, the protein also will not diffuse to the channel walls before leaving the device.

This approach is especially advantageous when combined with non-specific probing techniques such as SAXS where any additional material in the setup increases the background scattering and decreases the signal-to-noise ratio. It also reduces the width of the velocity distribution of the proteins by removing the low-velocity components near the channel walls as will be further discussed in section 6.1.3. This change in the flow field improves the temporal resolution for experiments using probes with an extended focal depth such as low divergence X-ray beams.

Further reduction of protein-wall contacts can be achieved by combining the reduced protein inlet height with an additional lateral separation layer as introduced in section 5.2 and depicted in figure 5.2. In the geometry shown in figure 5.3, the upstream edges of the intersection are in direct contact with both salt and protein and already slight instabilities in the flow can result in aggregation of protein at these lines. The insertion of an additional salt free buffer layer eliminates these contact lines and removes the last salt-protein-wall contact points resulting in an even better long-time stability of the flow.

\subsection{Accessing reactions in flow by SAXS}

Combining microfluidic mixers with X-ray scattering allows for the observation of reaction kinetics on a molecular level in solution. For such experiments, it is necessary to design microfluidic devices from materials with small cross-sections for X-ray absorption and scattering processes $[71,125,126]$. The devices used for these studies are usually custom-built to account for the experimental requirements, e.g. accessible scattering vectors or temporal resolution. Some possible materials and production methods for devices and their applications are presented in the following.

A variety of biomolecular processes, like collagen assembly or DNA condensation, have been studied in channels eroded in stainless steel, or molded in polydimethylsiloxane (PDMS) by soft lithography techniques that allow for more complex structures. The channels are covered by adhesive Kapton film [9,116,118,127, 128]. Pollack et al. investigated the dynamics of protein and RNA folding by SAXS $[60,119]$ using channels etched in silicon wafers and topped by thin, adhesive PDMS films [119]. Barrett et al. showed shear-induced transitions of worm-like micelles in channels ablated directly into Kapton foil [129]. Martel et al. chose an in-tube geometry 
of two thin borosilicate glass capillaries to mimic the formation of spider silk and to observe it in situ [130,131].

For SAXS experiments using the channel geometry introduced in sec. 5.3 and fig. 5.3 the device needs to fulfill the following requirements:

- weak SAXS signal from the device material, not given by Kapton adhesive film and PDMS

- complex geometries, like serpentine channels, realizable, given by soft lithography but not by erosion and ablation

- the reduced height of the protein inlet requires alignment and tight bonding of different parts of the channel, not given by Kapton foil or steel

- the material should be transparent in the visible spectrum, only partially given by either Kapton adhesive film or Kapton foil

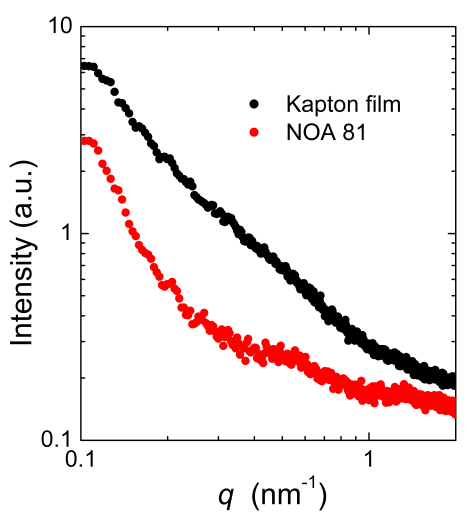

Figure 5.4: The SAXS signals of adhesive Kapton film and a thin film of NOA 81.

We found that Norland Optical Adhesive 81 (NOA 81, Norland, Cranberry, NJ, USA), a moldable, UV-curable, adhesive material fulfills the productional requirements $[132,133]$. Further, the SAXS signal of a thin NOA 81 film is small in comparison to adhesive Kapton foil (Dr D. Müller GmbH, Ahlhorn, Germany), as we saw in test experiments performed with a Bruker AXS Nanostar setup, including a rotating anode $\mathrm{X}$-ray source for $\mathrm{Cu}-\mathrm{K} \alpha$ radiation $(8 \mathrm{keV})$ at a generator power of $4.05 \mathrm{~kW}$ and a virtually noise-free, real-time 2D Hi-Star detector with photon counting ability (Bruker-AXS, Karlsruhe, Germany), courtesy of Thomas Pfohl (figure 5.4). Therefore, NOA 81 is a suitable material for studying the vimentin self-assembly in microflow by SAXS. 


\subsection{Fabrication of microfluidic devices}

For ease of production of the three-dimensional structures, we produce two symmetric halves of the devices which are then cured together. The main steps of the fabrication process are illustrated in figure 5.5. The geometry of the device is transferred to a photo resist layer on a silicon wafer using photolithography. From this master structure, a soft cast is produced, which serves as master structure for the casting of NOA 81 films. The two NOA 81 films are then joined to form the device and connected to tubing via a PDMS block.

\subsubsection{Photolithography}

The first step of the device fabrication is the production of a master wafer by photolithography, a well established technique for the production of single-layer structures $[134,135]$. In principle, since the NOA 81 is not molded directly from the wafer, there are two possible topologies: a closed resist layer with valleys or ridges of resist on the wafer. The first topology requires less consequent processing steps to obtain a mold for the NOA 81, but requires a higher quality resist layer and removal of uncured resist from the valleys. This removal is challenging for thinner channels (100 $\mu \mathrm{m}$ or less structure width in $120 \mu \mathrm{m}$ resist height), therefore this approach was only used for $300 \mu \mathrm{m}$ channels. For the fabrication of multi-layer structures as required for the step in channel height for the protein inlet, some adaptions of the standard protocols are necessary, which will be elaborated here for the case of valleys in a resist layer, see figure 5.5 a-d.

To produce even surfaces without impurities, the photolithography is performed in a class 100 cleanroom. In total, two layers of SU-8 negative resist (SU-8 3050, MicroChem, Newton, MA, USA), $65 \mu \mathrm{m}$ and $55 \mu \mathrm{m}$ thick, respectively, are spincoated onto the silicon wafer at $1800 \mathrm{rpm}$ (Optispin SB20, ATMgroup, Salem/Beuren, Germany) each. In order to prevent dewetting effects during the soft-baking process, the resist is slowly ramped to its final bake temperature: After applying the first layer, the temperature is ramped at $2 \mathrm{~K} / \mathrm{min}$ to $90{ }^{\circ} \mathrm{C}$, and after applying the second layer at $1 \mathrm{~K} / \mathrm{min}$ to $80{ }^{\circ} \mathrm{C}$. The baking times at the final temperature are 45 minutes and 30 minutes, respectively. The first layer is exposed to UV-light through photomask A, the second layer through photomask B (fig. 5.5k; mask produced by JD Photo-Tools, Oldham, UK; mask designed with AutoCAD 2008, München, Germany). Mask B is aligned to the existing structure on the wafer using a mask aligner (MJB4, Suess MicroTec, Garching, Germany). Between the application of the two 
a

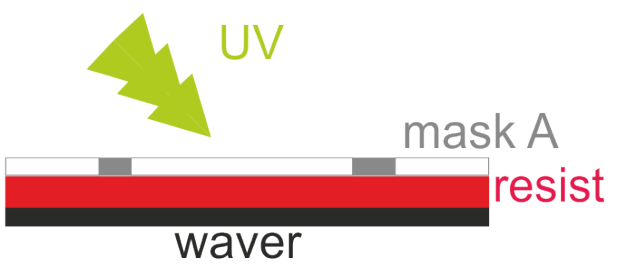

b

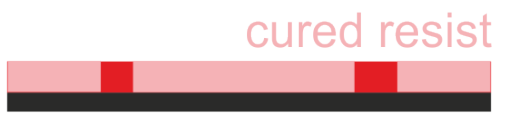

C

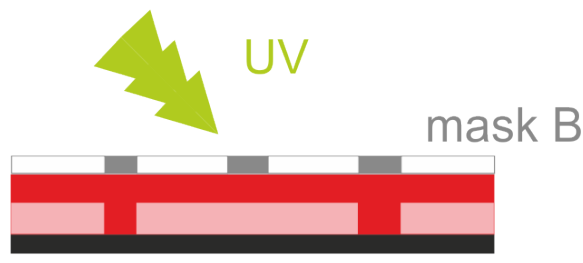

d
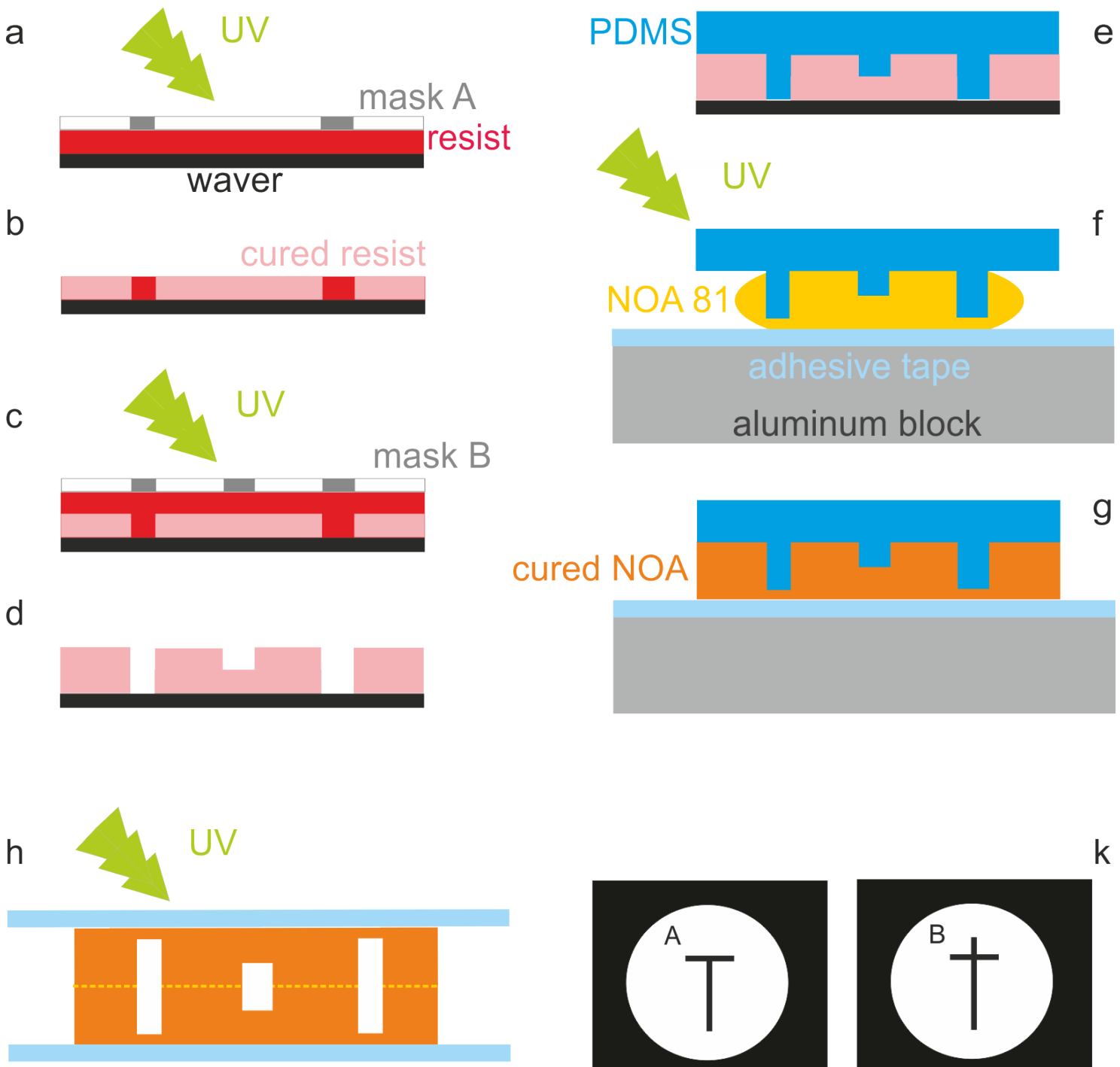

9
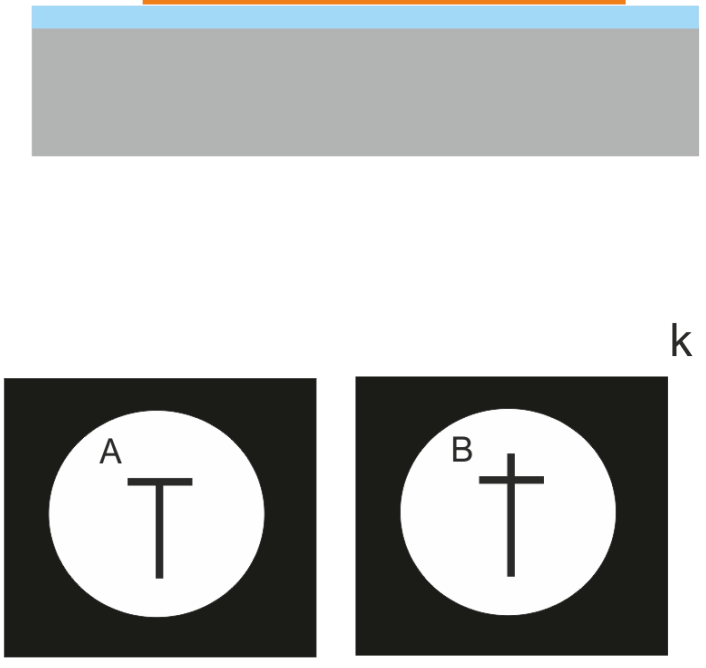

i

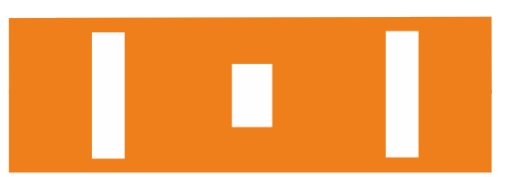

Figure 5.5: Schematic overview of device construction: a)-d) Two layers of photoresist are consecutively spincoated on a wafer and exposed to UV-light. After the second exposure, the resist is developed and previously non-exposed regions become channels in the resist. e) A PDMS-stamp is cast from the wafer. f), g) The stamp is placed on a drop of NOA 81 which is then UV-cured. h), i) Two films of NOA 81 are aligned and cured together. k) Schematic of the photomasks used in steps a) and c). The letters A and B indicate the two different masks used in the two-layer lithography process. 
layers, the wafer is baked for 1 minute at $65{ }^{\circ} \mathrm{C}$ and 7 minutes at $95{ }^{\circ} \mathrm{C}$, but no development process takes place. After the second exposure the resist is again baked for 1 minute at $65{ }^{\circ} \mathrm{C}$ and at $95{ }^{\circ} \mathrm{C}$ for 10 minutes, and finally developed in SU-8 developer. The resulting master is coated with (heptafluoropropyl)-trimethylsilane (Aldrich, Steinheim, Germany). Assymetric geometries, such as serpentines require two master wafers which mirror each other in oder to produce two corresponding halves of the device in subsequent steps. An exemplary master wafer for a serpentine geometry is shown in figure 5.6a.

For producing ridges instead of valleys, the photomasks need to be inverted and the order of the photomasks in the exposure steps is B-A instead of A-B.

(a)

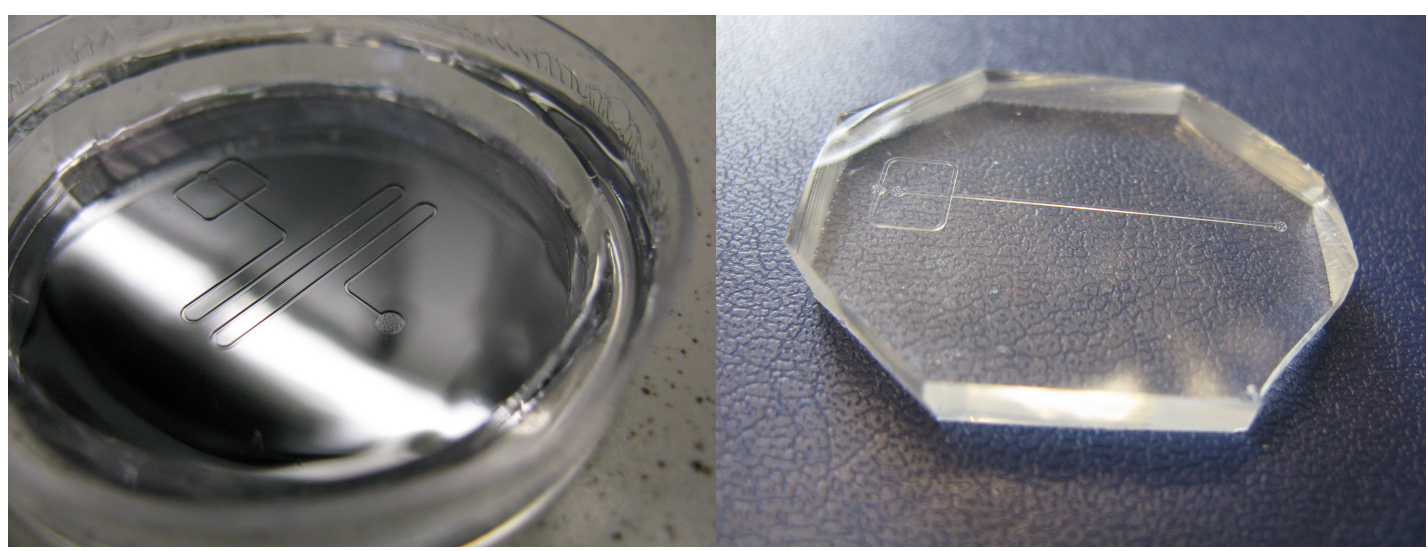

Figure 5.6: (a) Image of an examplary master wafer for a serpentine geometry with a channel width of $300 \mu \mathrm{m}$. (b) Image of an examplary PDMS stamp for a simple straight channel with a channel width of $300 \mu \mathrm{m}$.

\subsubsection{Casting of devices from the master wafer}

Following the production of master structures, suitable molds for the NOA 81 need to be fabricated. The direct molding of NOA 81 on SU-8 structures is possible, but removal of the NOA 81 films from the wafer is difficult as the films tend to bristle. Therefore we use PDMS stamps which are flexible themselves and can easily be removed from the NOA 81 films. Also, as PDMS is transparent up to $240 \mathrm{~nm}$ the NOA 81 can be exposed through the PDMS stamp [135]. An additional advantage of the PDMS stamps is that PDMS is permeable to oxygen which prevents complete curing of the NOA 81 at the interface $[136,137]$. This incomplete curing allows us to join the two halves without the need for any additional adhesives by completing the curing process. 
PDMS and crosslinker (Sylgard 184, Dow Corning, Midland, MI) are mixed at a ratio of $8: 1$ to create firm, yet flexible stamps. The mixture is poured on a wafer produced by photolithography as described above and baked for $2 \mathrm{~h}$ at $65{ }^{\circ} \mathrm{C}$ (fig. 5.5e). When the desired channel structure is present as valleys on the wafer, this PDMS cast can directly serve as stamp; figure $5.6 \mathrm{~b}$ shows an example of such a PDMS stamp. Otherwise this cast is transformed into a master for a second PDMS cast by plasma treatment (30 s in a plasma cleaner; PDC-32G, Harrick Plasma, Ithaca, New York, USA) and subsequent application of a hexamethyldisiloxane (HMDS, Merck, Darmstadt, Germany) coating by vapor deposition which prevents the PDMS for the second cast to cross-link with the original cast [138].

$100 \mu \mathrm{l}$ of NOA 81 are placed on the structure on the stamp. Air bubbles are carefully

(a)

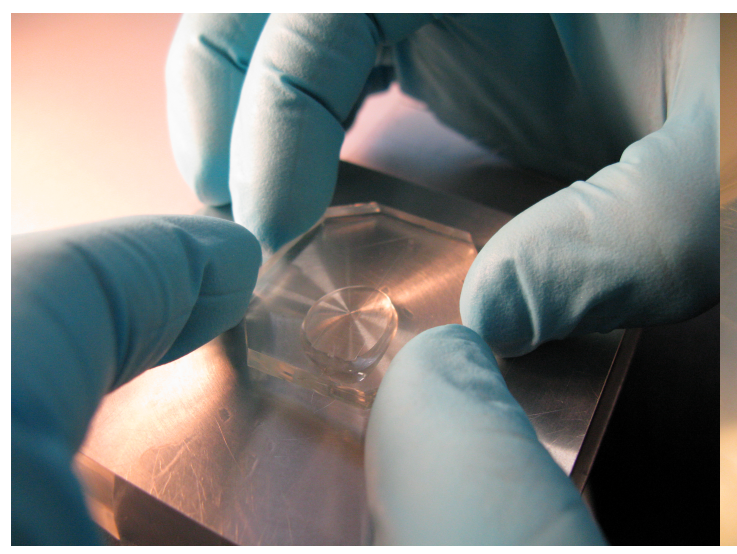

(b)

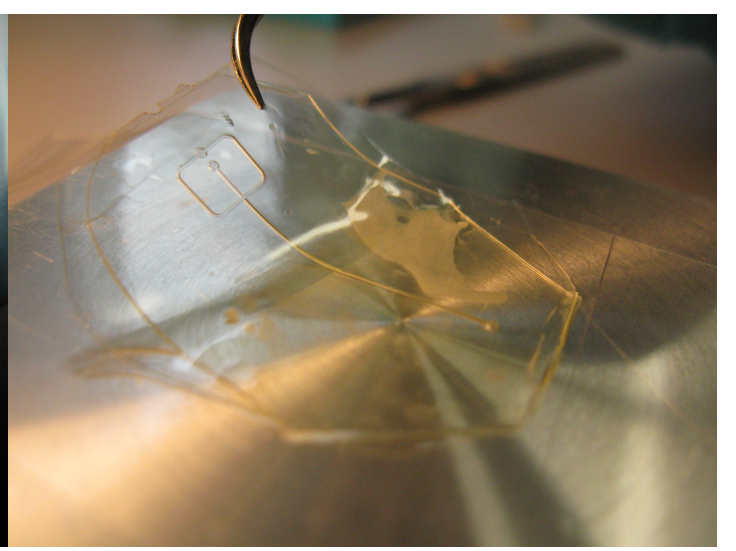

Figure 5.7: (a) A PDMS stamp is carefully placed on a drop of NOA 81 on an adhesive tape. (b) Removal of a completely cured device from the adhesive tape.

removed with a thin pipette tip. Subsequently, another $180 \mu \mathrm{l}$ of NOA 81 are applied on a piece of adhesive tape (tesa Pack, tesa SE, Hamburg, Germany) attached to an aluminum block used as solid support. The transparent adhesive tape is a good base for the alignment process and can be easily removed after the final curing of NOA 81. Then, the stamp is placed on the adhesive tape as shown in fig. 5.5f. When the spreading NOA 81 film completely covers the structure, it is exposed to UV-light ( $8 \mathrm{~W}$ at $365 \mathrm{~nm}, 1 \mathrm{~min}$; lamp: NU-8 LK, Benda Ultraviolettstrahler, Wiesloch, Germany) (fig. 5.5g). The PDMS stamp is removed and the NOA 81 film remains on the adhesive tape. A second NOA 81 film is produced in exactly the same way. Both films are placed on top of each other, with the structures facing each other (fig. 5.5h). Further exposure to UV-light ( $8 \mathrm{~W}$ at $365 \mathrm{~nm}, 10 \mathrm{~min}$ ) cures them together (fig. 5.5i). The resulting films are about $500 \mu \mathrm{m}$ thick. The buffer inlet channels and the outlet channel are $240 \mu \mathrm{m}$ high, the central inlet is $110 \mu \mathrm{m}$ 
high. All channels are $300 \mu \mathrm{m}$ wide. The geometry of the channels was controlled by imaging cuts through the device with a microscope (IX71, Olympus, Tokyo, Japan).

\subsubsection{Connection to the syringe system}

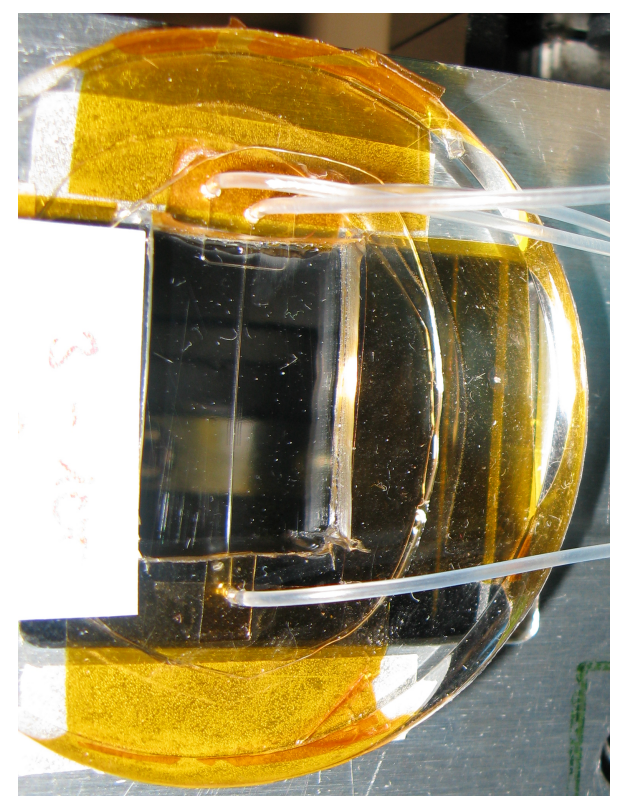

Figure 5.8: A microfluidic device from NOA 81 complete with tubing mounted for measurements at cSAXS. The inlets are covered by PDMS to attach the tubing but the mixing region and the channel downstream are uncovered.
The channels in the NOA 81 films need to be connected to the syringe system. For this, a stable connection with little dead-volume is required. We connect our devices via a flexible PDMS layer as follows: PDMS and crosslinker are mixed at a ratio of 10 : 1 and cast into disks of $5 \mathrm{~cm}$ diameter and $4 \mathrm{~mm}$ height $(2 \mathrm{~h}$ baking time, $65{ }^{\circ} \mathrm{C}$ ). The PDMS is glued to the structured NOA 81 film with Loctite Polyolefin (Loctite 406 and 770, Henkel, Düsseldorf, Germany), leaving measurement windows at the position of the regions of interest in the device. Before the Loctite completely hardens, holes for the inlets and outlets are punched through the NOA 81 film and the PDMS. The holes on the NOA 81 side are closed with Kapton tape (Dr D. Müller GmbH, Ahlhorn, Germany) which provides a reliable, tight closure. On the PDMS side polyethylene tubing (inner diameter $380 \mu \mathrm{m}$, outer diameter $1.09 \mathrm{~mm}$; INTRAMEDIC CLAY ADAMS

Brand, Becton Dickinson and Company, Sparks, Maryland, USA) is placed into the holes and connected to the syringes (Hamilton Gastight, Hamilton Company, Reno, Nevada, USA). The syringes are operated by a remote-controllable pumping system (Nemesys, Cetoni, Korbußen, Germany). 


\subsection{Characterization of solute distribution with fluorescein}

As discussed in section 5.3 the smaller protein inlet prevents protein-channel contacts because the diffusion of the protein is too slow to cross the buffer layer before the end of the device. This of course is only true if the Péclet number is sufficiently high. As the geometry of the flow is given by the ratio of the flow speeds of protein, and buffer and the diffusion constant of vimentin is set, the remaining question is the minimal required flow speed.

To address this question, we pump a $100 \mathrm{mM}$ fluorescein solution into the central inlet, and water into the side inlets. We observe the resulting fluorescein concentration at various flow speeds between $193 \mu \mathrm{m} / \mathrm{s}(50 \mu \mathrm{l} / \mathrm{h})$ and $25 \mathrm{~mm} / \mathrm{s}(6500 \mu \mathrm{l} / \mathrm{h})$ by confocal microscopy (Olympus Fluoview 1000 CLSM equipped with a UPLSAPO 10× objective; Olympus, Tokyo, Japan) and compare the results to finite element method (FEM) simulations (COMSOL multiphysics 4.0a; COMSOL GmbH, Göttingen, Germany), see fig. 5.9. The flow rate of the fluorescein solution was half that of the water at each inlet, resulting in nearly equal flow speeds. For the simulations the diffusion of fluorescein was set to $4.25 \cdot 10^{10} \mathrm{~m}^{2} / \mathrm{s}$ [139]. The variation of the flow speeds allows us to determine the effects of diffusion on the spatial distribution of the solute. As the distributions at higher flow speeds correspond to those of proteins with a diffusion constant of about an order of magnitude less, we will focus on the results for these speeds. The simulations assist in the interpretation of the data and allow us to distinguish between convection and diffusion effects. We find that the fluorescein flow gets focused in the $x-y$ plane upon entry of the cross. At low flow speeds an immediate broadening of the flow due to diffusion is observed as shown in figure 5.9a. We further find that for all flow speeds the fluorescein distribution expands in z-direction perpendicular to the main flow direction directly after entering the intersection (figure 5.9b,c). For high flow speeds this expansion can be solely attributed on the turning of the streamlines as depicted in figure 5.9c. At lower flow speeds, the distribution is further broadened by diffusion. At a high average flow speed of $12.5 \mathrm{~mm} / \mathrm{s}(3250 \mu \mathrm{l} / \mathrm{h})$ the concentration profile along the z-axis in the middle of the intersection is relatively constant in the center of the jet and then rapidly vanishes (figure 5.9d). This corresponds well to the simulated concentration profile. Note that in the experiment the value does not decrease to zero due to the finite size of the confocal volume while in the simulation a similar effect is observed, which is due to the size of the finite elements. In general, the observed concentration 
a
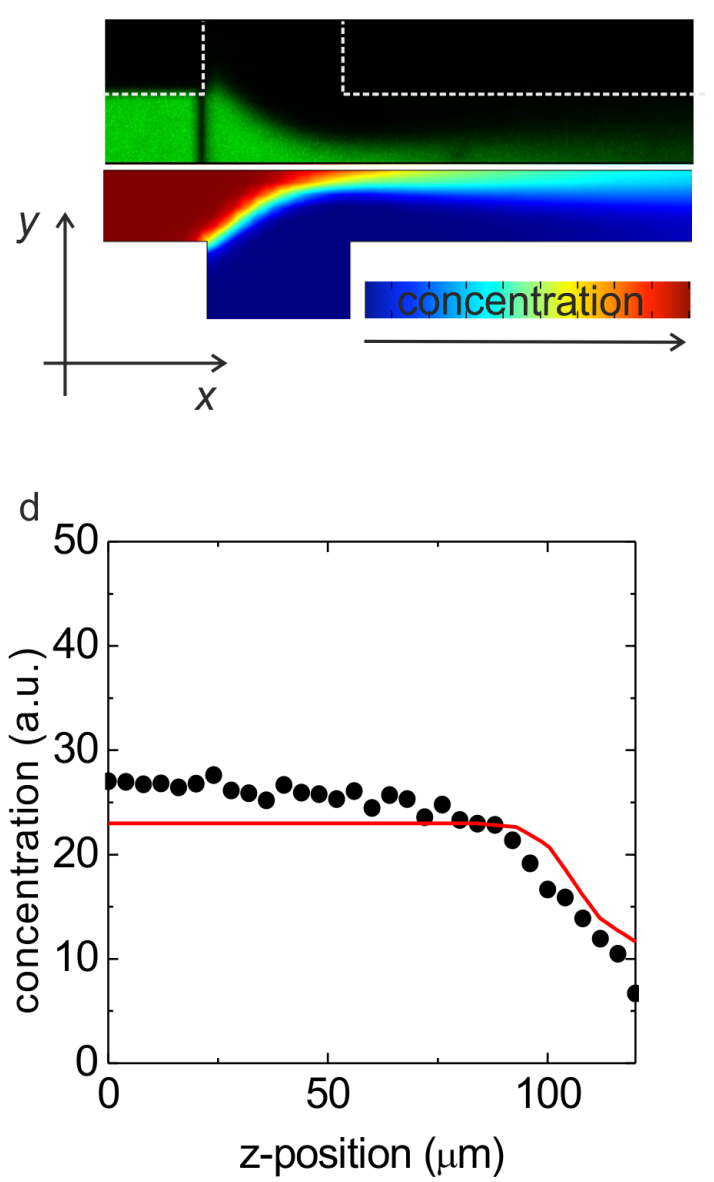

b

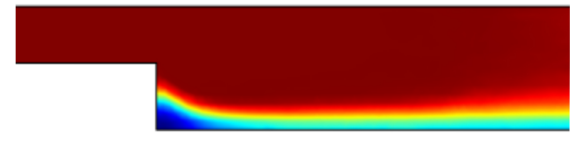

C
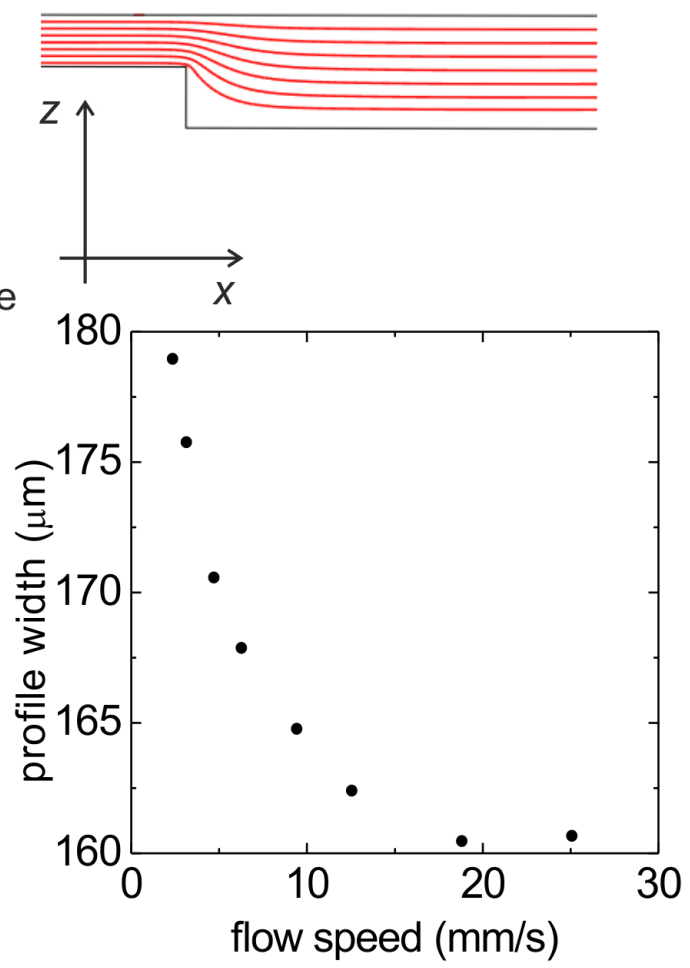

Figure 5.9: Distribution of fluorescein in the device. a) Comparison of experiment and simulation, top-view: fluorescein distribution in the midplane of the device at $193 \mu \mathrm{m} / \mathrm{s}$ $(50 \mu \mathrm{l} / \mathrm{h})$ flow. The fluorescein diffuses towards the channel walls as it flows downstream. The channel walls are marked by dashed lines. b) Simulation of the fluorescein distribution, view on the central $x-z$ plane at $12.5 \mathrm{~mm} / \mathrm{s}(3250 \mu \mathrm{l} / \mathrm{h})$ flow. c) View on the central $x-z$ plane of the simulated streamlines at the intersection at $12.5 \mathrm{~mm} / \mathrm{s}(3250 \mu \mathrm{l} / \mathrm{h})$ flow. The streamlines from the central inlet turn downwards but do not reach the bottom of the channel. d) z-profile of the fluorescein distribution at the center of the intersection, at $12.5 \mathrm{~mm} / \mathrm{s}(3250 \mu \mathrm{l} / \mathrm{h})$ flow. Line: simulation, circles: experiment. e) The width of the z-profile of the fluorescein distribution $2 \mathrm{~cm}$ downstream of the intersection decreases with the flow speed. 
distributions are in good agreement with the simulation. At sufficiently high flow speeds the fluorescein remains constricted to a thin sheet formed by the streamlines even further downstream as diffusion effects remain negligible. At lower flow speeds the fluorescein distribution in both the $y$ - and $z$-direction broadens to eventually fill out the complete channel width and height (figure 5.9a). We observe that as we decrease the flow speed the $z$-profile becomes more stepwise and flat. This can be quantified by determining the width of the fluorescein distribution perpendicular to the flow at roughly $2 \mathrm{~cm}$ downstream from the intersection by calculating its standard deviation from the center (figure 5.9e). For the highest observed flow speeds, the distribution is relatively slim as there is little diffusion. As the flow becomes slower, the increasing influence of diffusion broadens the profile until it fills up the complete height of the device and fluorescein reaches the channel walls.

These results show that for fluorescein miminal flow speeds that ensure abscence of channel wall contact are in the range of $10 \mathrm{~mm} / \mathrm{s}$. For large proteins whose typical diffusion constants are an order of magnitude or more smaller than the diffusion constant of fluorescein, similar concentration distributions are achieved at identical Péclet numbers. As the typical length scale remains constant, this is equivalent to an identical flow speed to diffusion constant ratio and it follows that protein-channel wall interactions are absent for total flow speeds of about $1 \mathrm{~mm} / \mathrm{s}$ when the flow speeds in the inlet are roughly equal.

\subsection{Assembling vimentin in microflow}

This section discusses the technical aspects of letting vimentin assemble in mircoflow as described in section 5.1.2, especially how to ensure stable flow conditions and how to control for these.

The main obstacles to stable flow are air bubbles and uncontrolled protein-salt contact resulting in adhesion of protein. To prevent air bubbles it is vital to use bubble free buffer and protein solutions. To achieve this requirement, all fluids are transferred to room temperature $1 \mathrm{~h}$ before experiment and thoroughly de-gased before being filled into the syringes. Bubbles emerging when filling the syringes (usually in the connection between the hollow needle and the syringe body) are carefully removed. Before tubings are attached to the device, they are filled with fluid until a small droplet forms at their tip so that no bubbles enter at this step. Finally, the protein inlet is sealed for buffer measurements, which are necessary to separate the scattering from the protein from the scattering from the buffer and the device, and 
before attaching the tubing for the protein solution, the protein inlet is completely filled with buffer.

To prevent protein solution from entering the buffer inlets, the flow rate of the buffer is set to at least twice the flow rate of the protein when connecting the protein tubing and no tubing is attached to the outlet to minimize the hydraulic resistance. After changing flow speeds, the system is given at least 10 min to equilibrate before measurements are started.

Differential interference contrast (DIC) images of the protein flow, which show stable flow over several hours, were recorded with a motorized inverted research microscope (IX81, Olympus, Tokyo, Japan) equipped with an UPLFLN 20X objective (Olympus, Tokyo, Japan). At cSAXS (see section 3.3.2), stable flow conditions can be controlled with a $10 \times$ microscope at a fixed location from the X-ray focus. This microscope also enables us to probe the microflow at well-defined positions. 


\section{Chapter 6}

\section{Modeling of SAXS curves from vi- mentin assembly in flow}

In this chapter, we use simulations of the flow of vimentin to understand how it depends on the device geometry, flow speeds and buffer salt concentrations. Based on those results and the stationary SAXS data described in chapter 4, SAXS curves for different measurement locations are estimated. These results assist in interpreting the experimental SAXS curves of vimentin in microflow (chapter 7).

\subsection{Simulation of flow field and concentration dis- tributions}

As discussed above (sec. 5.1), the flow in microfluidic devices is laminar. This allows us to access the flow fields and concentration distributions in the device by finite element method (FEM) simulations. FEM is an approximation method for partial differential equations (PDEs) for a given geometry and border conditions. It divides the geometry into sub-volumes (mesh) on which local approximations based on linear combination of Ansatz-functions can be calculated (finite elements). Continuity between the elements is ensured by combining all elements into a global linear equation system which can be solved for the given boundary conditions [140]. We use COMSOL multiphysics 4.0a (COMSOL GmbH, Göttingen, Germany) to perform FEM simulations of the flow in our microfluidic devices. 


\subsubsection{Simulation system}

Our goal is to describe the distribution of solutes and the flow field in a device under different geometries, flow rates and buffer salt concentration, in order to understand the ensemble of vimentin conditions, i.e. all different local environments, which contribute to the SAXS signal at different locations of the device. The relevant PDEs for this problem are the Navier-Stokes equation (equation 5.1) for the flow field and the convection-diffusion equation (equation 5.8) for the solutes. These equations are coupled to each other: The dependence of the convection-diffusion equation on the solution of the Navier-Stokes equation is straightforward. The other direction of the coupling follows from the fact that the viscosity of the fluid depends on the local protein and salt concentration and the assembly state of the protein, and therefore on the solute distribution $[23,32,36]$. Therefore, both equation systems need to be solved simultaneously.

\section{Model geometry and boundary conditions}

Our goal is to describe the geometry introduced in figure 5.3, i.e. a cross-geometry with a channel width of $300 \mu \mathrm{m}$, buffer inlets and an outlet with a height of $240 \mu \mathrm{m}$, a central protein inlet with a height of $110 \mu \mathrm{m}$, which is centered with respect to the other channels, and a total length of the device of several centimeters. We define the $x$-axis parallel to the protein inlet and outlet, the $y$-direction parallel to the buffer inlets and the $z$-direction perpendicular to those. The point $(0,0,0)$ corresponds to the middle of the intersection of the protein and buffer inlets.

The smallest relevant structure in this geometry is the difference in channel height at the intersection of the protein inlet and the buffer inlets with a relevant size of $65 \mu \mathrm{m}$ corresponding to half the height difference. When aiming to construct the mesh such that this step is divided into several sub-units, it becomes obvious that we need to reduce the simulated area of the device. First, the existence of two symmetry axes in all device layouts allows us to reduce the computational effort by modeling only a quarter of the respective device (cut in the $x-z$ plane and the $y$ - $z$ plane). Second, we reduce the length of the inlet channels to $500 \mu \mathrm{m}$ and the length of the outlet channel to $1 \mathrm{~mm}$. This is not only suffiently large to ensure the correct flow profiles in the inlets but also covers most of the mixing region (see also section 6.1.3). The tetrahedral mesh size which varies throughout the simulation volume, is computed by COMSOL with a minimum mesh size of $0.3 \mu \mathrm{m}$ and a maximum mesh size of $7.5 \mu \mathrm{m}$. 
We choose the laminar flow simulation mode, the channel walls are set to fulfill the no slip condition, at the inlets the velocity is fixed at a constant value and at the outlet the boundary condition is "pressure, no viscous stress". The solutes are set to a fixed concentration at the inlets (either zero or their bulk concentration).

\section{Global constants and modeling of the viscosity}

The diffusion constant of tetrameric vimentin is estimated to be $D_{\text {vim }}=4.1$. $10^{-11} \mathrm{~m}^{2} / \mathrm{s}$ [141]. This estimate for the diffusion constant does not account for the non-spherical shape of the assemblies. For diffusion perpendicular to the long axis of the cylinder-shaped tetramers, the value will be slightly decreased, while it increases (by a factor of 2 at most), when assuming diffusion parallel to the long axis. Furthermore, the tetramer diffusion constant is the largest diffusion constant found in our system since larger assemblies (e.g. ULFs) have smaller diffusion constants. To reduce the computational effort, we neglect this decrease of the diffusion constant due to the filament growth during assembly and assume this diffusion constant, which poses an upper boundary for the diffusion of larger aggregates throughout the entire assembly. The diffusion of potassium chloride is modeled with $D_{K C l}=1.84 \cdot 10^{9} \mathrm{~m}^{2} / \mathrm{s}[142]$. We assume that the diffusion of salt ions into the vimentin jet is not influenced by the presence of the protein assemblies, in accordance with previous work $[116,127]$, i.e. the diffusion constant has a fixed value.

It has been reported that after the addition of salt to the vimentin solution the viscosity increases and approaches a final value on the timescale of minutes $[23,36]$. This time-dependence of the viscosity can either be due to the intrinsic reaction speed or limited by the diffusion speed of the salt ions. We assume that the assembly is diffusion limited in bulk and that the increase of the viscosity on the microfluidic length scale is likely to be instantaneous. Therefore, we only consider the final viscosities observed in these experiments. We model the slight increase in viscosity of vimentin solution as compared to water or buffer both for tetramer solution $\left(\eta_{t e t}=1.2 \mathrm{mPas}\right)$ and upon assembly of the vimentin $\left(\eta_{v i m}=2.8 \mathrm{mPas}\right)$, to replicate the dependence of the viscosity on salt and protein concentrations given in [32] with

$$
\eta=\eta_{H_{2} O} \cdot\left(1+\eta_{v} c_{v i m}+\eta_{s} \Theta\left(c_{v i m}-c_{m i n}\right)\left(1-\frac{1}{1+\left(c_{K C l} / c_{0}\right)^{p}}\right)\right)
$$

where $\eta_{H_{2} O}$ is the viscosity of water, $c_{v i m}$ and $c_{K C l}$ the local concentrations of vimentin in $\mathrm{mg} / \mathrm{ml}$ and salt in $\mathrm{mM}$ respectively in accordance with [32], $\eta_{v}=$ 
$0.04053 \mathrm{ml} / \mathrm{mg}$ describes the increase of viscosity due to tetrameric vimentin and $\eta_{s}=1.8$ is the maximal increase of viscosity due to filamentous vimentin. The latter requires the presence of vimentin which is modeled with a $\Theta$-function, which is non-zero only for vimentin concentration larger than $c_{\min }=0.1 \mathrm{mg} / \mathrm{ml}$, and also depends on the salt concentration, which is modeled with a logistic function with the parameters $c_{0}=55 \mathrm{mM}$ and $p=4.4$. The values of the parameters are chosen to reproduce the results in [32].

\subsubsection{From simulations to finite probe volumes}

From the simulations we can directly obtain local flow velocities and concentrations at any given point. In a SAXS experiment, however, the recorded signal is always the superposition of all states in the X-ray beam passes, i.e. when probing at the location $(x, y)$ of the device with a spot size $(\Delta x, \Delta y)$

$$
I(q, x, y, \Delta x, \Delta y) \propto \iiint_{V} \mathrm{~d} x^{\prime} \mathrm{d} y^{\prime} \mathrm{d} z^{\prime} c_{v i m}\left(x^{\prime}, y^{\prime}, z^{\prime}\right) I_{l}\left(q, x^{\prime}, y^{\prime}, z^{\prime}\right)
$$

where $c_{v i m}\left(x^{\prime}, y^{\prime}, z^{\prime}\right)$ is the vimentin concentration at $x^{\prime}, y^{\prime}, z^{\prime}, u\left(x^{\prime}, y^{\prime}, z^{\prime}\right)$ the flow speed in $x$-direction, $I_{l}\left(q, x^{\prime}, y^{\prime}, z^{\prime}\right)$ the scattering intensity of the vimentin state at $x^{\prime}, y^{\prime}, z^{\prime}$. We also introduced $V$, the sample volume contributing to the signal, which is in the $x$-y plane defined by $(x, y)$ and $(\Delta x, \Delta y)$ and in the $z$-direction limited by the lower and upper boundary of the device parallel to the beam as typical focal lengths of X-ray beams are in the range of millimeters, see e.g. [143]. Assuming that the state of vimentin depends only on the current salt concentration $c_{s}$ and the time $t$ passed since the first contact with salt ("assembly time"), equation 6.2 becomes

$$
I(q, V) \propto \iiint_{V} \mathrm{~d} x^{\prime} \mathrm{d} y^{\prime} \mathrm{d} z^{\prime} c_{v i m}\left(x^{\prime}, y^{\prime}, z^{\prime}\right) I_{l}\left(q, c_{s}\left(x^{\prime}, y^{\prime}, z^{\prime}\right), t\left(x^{\prime}, y^{\prime}, z^{\prime}\right)\right)
$$

The salt and protein concentrations are direct results of the simulation, but in order to determine the assembly time it is in principle necessary to track each volume element of protein as it flows through the device. As the diffusion of vimentin is rather weak, we can assume that it mostly follows the stream lines which can be directly calculated in COMSOL. The assembly time can then be estimated by integrating over the inverse of the flow velocity along the streamline starting with a minimal salt concentration which we set to $1 \mathrm{mM}$. Once the assembly times throughout the device have been calculated, extraction of $c_{v i m}, c_{s}$ and $t$ for any probe volume ele- 
ment is straightforward, for details see appendix D.

The calculated streamlines begin at $x=-400 \mu \mathrm{m}$ with a spacing of $1 \mu \mathrm{m}$ in both $y$ and $z$. The resolution in COMSOL was set to "extrafine" which corresponds to nearly three discrete points per $\mu \mathrm{m}$. The calculations were performed in MATLAB R2009b, using the LiveLink to MATLAB provided by COMSOL for streamline calculation and simulation evaluation along the streamlines. The employed code is given in appendix D.

\subsubsection{Exemplary simulation results}

Without any further analysis of the simulation results some basic questions can already be answered by looking at the concentrations of vimentin and potassium chloride in cut planes throughout the simulation volume. In figure 6.1 such concentration distributions are given for a buffer salt concentration of $200 \mathrm{mM} \mathrm{KCl}$ and a protein concentration of $4 \mathrm{mg} / \mathrm{ml}$ with corresponding mean flow speeds in the inlets of $310 \mu \mathrm{m} / \mathrm{s}(80 \mu \mathrm{l} / \mathrm{h})$ and $335 \mu \mathrm{m} / \mathrm{s}(40 \mu \mathrm{l} / \mathrm{h})$ respectively.

The first question is whether or not the simulated concentration distributions correspond to reality. To address this, we compare the vimentin concentration from the simulation in the mid $x-y$ plane $(z=0)$ of the device to DIC microscopy images of the same plane, see figure 6.1a. In DIC, the difference in the index of refraction between the buffer and the protein solution marks the location of the protein jet. We see that this line corresponds nicely to the protein concentration obtained in the simulations. The width of the vimentin jet estimated from the experiment is about $75 \mu \mathrm{m}$, which is larger than the $60 \mu \mathrm{m}$ the simulation predicts. However, as device alignment is never perfect, a larger jet width could be expected.

The next question we can address is whether the simulations confirm the existence of a buffer layer above and below the vimentin, as stated in section 5.3 and confirmed by the long term stability of flow. This is easily confirmed by considering the vimentin distribution in any $y$ - $z$ plane after the beginning of the intersection (i.e. for $x>-150 \mu \mathrm{m})$. The right hand side of figure 6.1c shows the vimentin distribution in the middle of the intersection, i.e. at $x=0$. We see that indeed the vimentin concentration at the top and bottom of the device is zero which means that we correctly predict the absence of vimentin to channel wall contacts.

Finally, we can address our argument on the differences of the salt and vimentin distributions based on their respective diffusion constants (section 5.2). Figure 6.1b shows a comparison of the distributions for the mid-plane $(z=0)$ of the device and figure 6.1c shows the comparison for the mid $y$-z plane of the intersection $(x=0)$. 


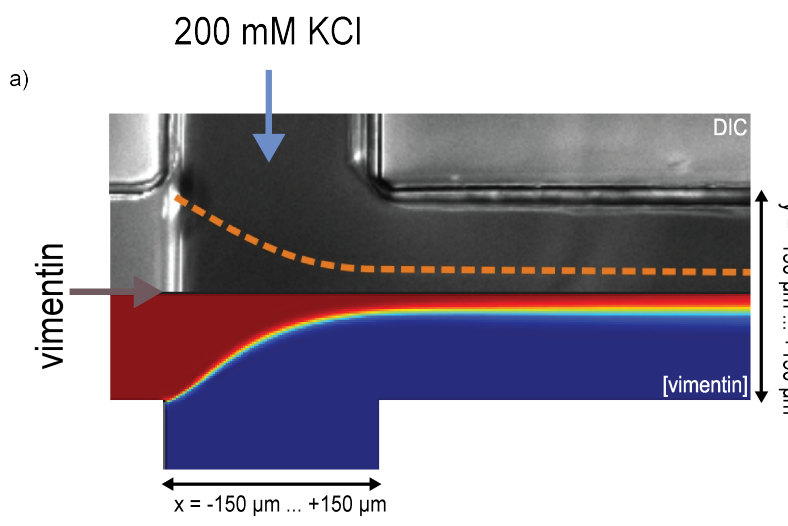

b)

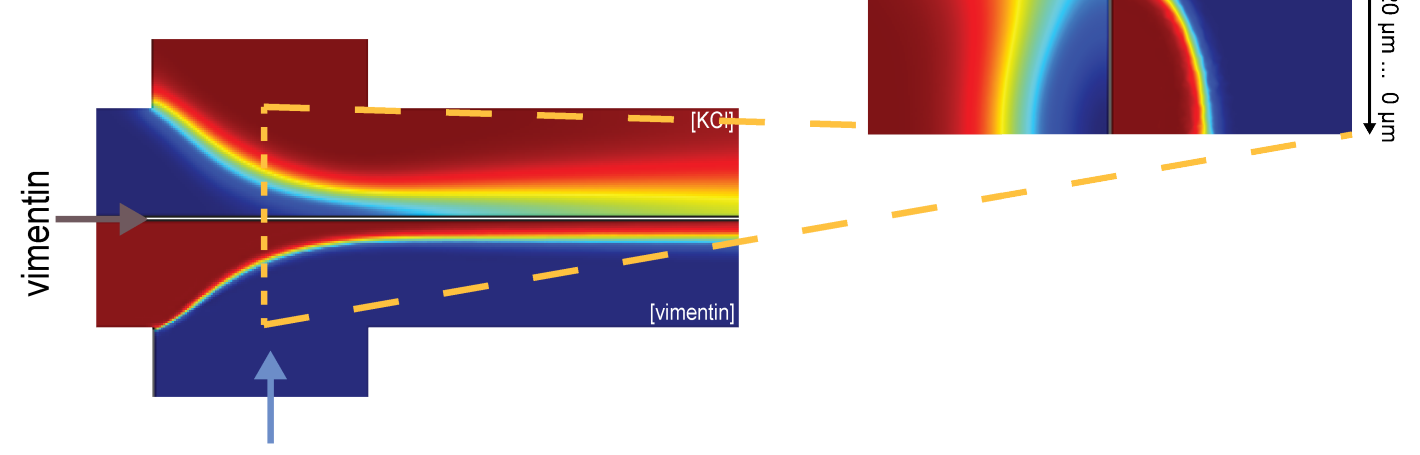

$200 \mathrm{mM} \mathrm{KCl}$

Figure 6.1: Results of the simulation of vimentin flow in the microfluidic devices. The mean flow speed of the vimentin solution $\left(c_{v i m}=4 \mathrm{mg} / \mathrm{ml}\right)$ in the inlet is $335 \mu \mathrm{m} / \mathrm{s}$ $(40 \mu \mathrm{l} / \mathrm{h})$ and the mean flow speed of the salt buffer solution in the inlet is $310 \mu \mathrm{m} / \mathrm{s}$ $(80 \mu \mathrm{l} / \mathrm{h})$. (a) Comparison of the vimentin concentration from the simulation in the mid $x$-y plane $(z=0)$ of the device (lower part) with a DIC micrograph of flow in the same plane (upper part). The dotted line in the DIC image marks the border of the vimentin jet, which matches well with the prediction from the simulation. (b) Comparison of the vimentin (lower part) and potassium chloride (upper part) concentrations in the same plane. The vimentin remains on a thin jet in the middle of the device into which the ions diffuse. (c) Comparison of the vimentin (right part) and potassium chloride (left part) concentrations in the mid $y$-z plane of the intersection $(x=0)$. The vimentin has no contact to the channel walls. The salt diffuses into the vimentin mostly from the sides. 
For the $x$ - $y$ plane we see that the vimentin mostly stays on a jet in the middle defined by the streamlines, whereas diffusion of the salt into that jet is noticeable from the beginning, with the concentration on the middle axis of the device already exceeding $100 \mathrm{mM}$ at $x=250 \mu \mathrm{m}$. In the $y-z$ plane we notice that the salt diffuses into the jet mostly from the sides and that the equi-concentration lines appear nearly parallel to the edges of the device. This implies that the distribution of concentrations when probing with a very small probe is rather narrow.

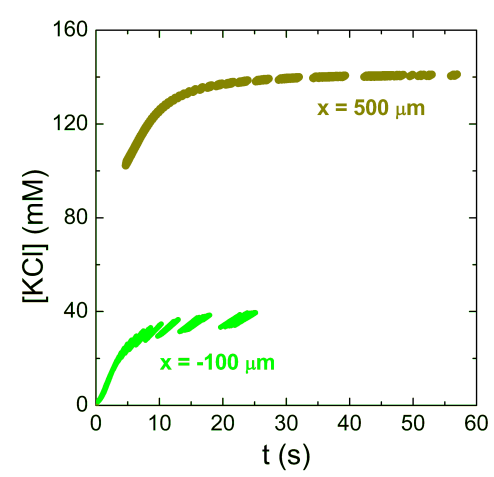

Figure 6.2: Ensembles of salt concentrations and assembly times at two different positions for flow conditions as in figure 6.1 .
To understand what we would probe in an experiment we consider the salt concentration and assembly time ensembles at different locations along the $y=0$ axis of the device for a spot size of $20 \mu \mathrm{m} \times 5 \mu \mathrm{m}(x \times y)$, which corresponds to the nominal beam size for our experiments at cSAXS (see section 3.3.2). Because, as discussed above, our SAXS signal depends on all individual states of vimentin found in the probe volume, and therefore on all combinations of salt concentrations and assembly times.

Figure 6.2 shows all salt concentration - assembly time combinations probed for two different positions along the flow. For $x=-100 \mu \mathrm{m}$, i.e.

$50 \mu \mathrm{m}$ into the intersection of the inlets, the salt concentration varies between 0 and $40 \mathrm{mM}$ and the assembly time between 0 and $25 \mathrm{~s}$. However, only very few points reach the higher values. For $x=500 \mu \mathrm{m}$, the salt concentrations vary between $100 \mathrm{mM}$ and $140 \mathrm{mM}$, i.e. the relative spread of the distribution with respect to its mean is decreased, and assembly times vary between $4.5 \mathrm{~s}$ and $1 \mathrm{~min}$. The highest assembly times correlate with the highest salt concentrations which is due to the fact that the salt diffuses into the protein jet from the direction of the channel walls, i.e. the highest salt concentrations are relatively close to the channel walls and there the flow speeds are the lowest. When we regard the histograms for the assembly times and salt concentrations at a fixed position, e.g. $\mathrm{x}=500 \mu \mathrm{m}$, we see that for the salt concentration the more extreme values (about $105 \mathrm{mM}$ and $140 \mathrm{mM}$ ) actually occur more often than the values between them. In contrast to that, smaller assembly times present the largest part of the ensemble and the decay of the histogram towards larger assembly times is rather fast (note the logarithmic scale of the plot). This behavior is understandable when we consider that the flow 
(a)

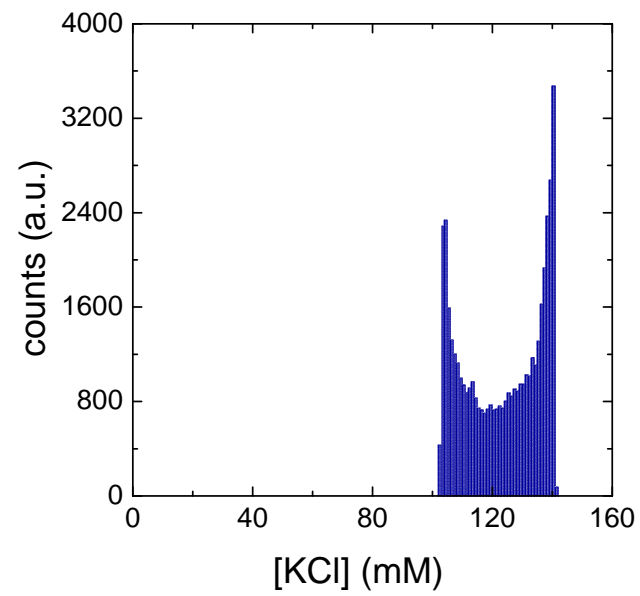

(b)

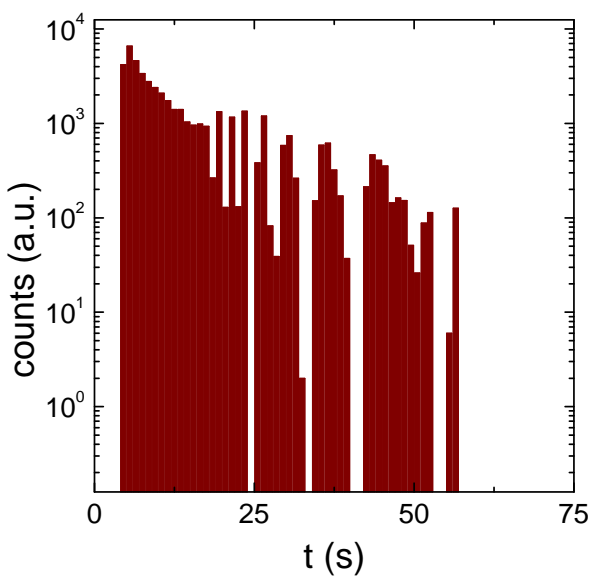

Figure 6.3: Exemplary histograms of (a) salt concentrations and (b) assembly times for a mean flow speed of the vimentin solution $\left(c_{v i m}=4 \mathrm{mg} / \mathrm{ml}\right)$ in the inlet of $335 \mu \mathrm{m} / \mathrm{s}$ $(40 \mu \mathrm{l} / \mathrm{h})$ and a mean flow speed of the salt buffer solution $([\mathrm{KCl}]=200 \mathrm{mM})$ in the inlet of $310 \mu \mathrm{m} / \mathrm{s}(80 \mu \mathrm{l} / \mathrm{h})$ sampled with a $20 \mu \mathrm{m} \times 5 \mu \mathrm{m}(x \times y)$ spot at $y=0$ and $x=500 \mu \mathrm{m}$.

speeds follow a parabola-like profile (Poiseuille flow) in both the $y$ - and $z$-directions and that by probing at $y=0$ with a $5 \mu \mathrm{m}$ broad X-ray spot the variation in the flow speed is small.

Based on these ensembles we can determine the minimum, mean and maximum salt concentrations and assembly times in each probed volume. In figure 6 .4a we see that before the intersection, the salt concentration is indeed 0 everywhere, but the maximum concentration increases fast afterwards, reaching $70 \mathrm{mM}$ in the middle of the intersection and $150 \mathrm{mM} 1 \mathrm{~mm}$ downstream. At the middle of the intersection, the minimum concentration reaches only $5 \mathrm{mM}$, and $1 \mathrm{~mm}$ further downstream it is $130 \mathrm{mM}$. Both curves apparently approach $160 \mathrm{mM}$ and become closer to each other. The mean value tends to be closer to the minimum value. Despite the large differences in the maximum and minimum concentrations, the standard deviation of the salt concentration distribution in the probe volume is less than $1 \mathrm{mM}$. Just as the salt concentrations, the assembly times are 0 before the intersection (figure $6.4 \mathrm{~b}$ ). The maximum assembly time however, is already $40 \mathrm{~s}$ in the middle of the intersection and just surpasses one minute at $x=1 \mathrm{~mm}$. These rather long times are due to the fact that at the top and bottom of the vimentin jet the flow speeds are very low and additionally the vimentin in these regions comes in contact with salt very soon after entering the intersection. In contrast, the minimum assembly 
(a)

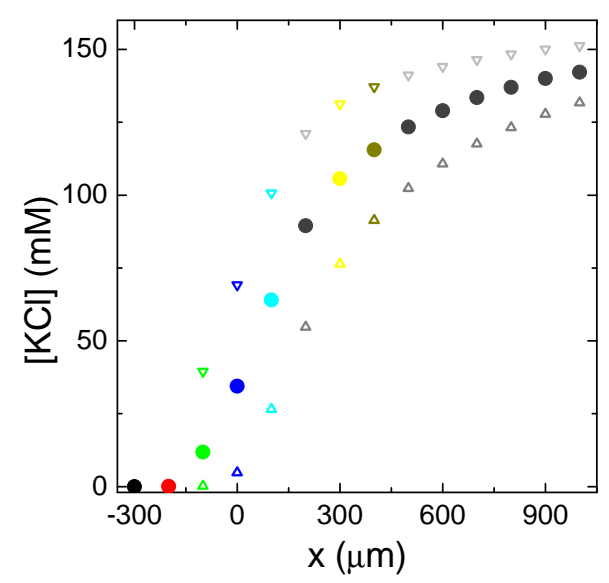

(b)

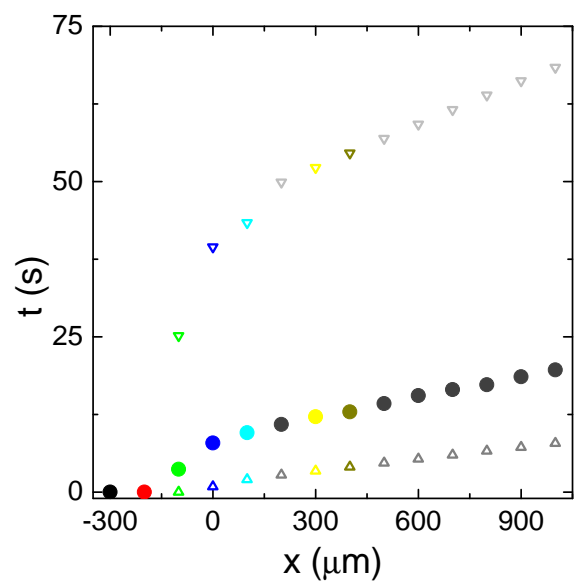

Figure 6.4: Exemplary salt concentrations and assembly times for a mean flow speed of the vimentin solution ([vimentin] $=4 \mathrm{mg} / \mathrm{ml})$ in the inlet of $335 \mu \mathrm{m} / \mathrm{s}(40 \mu \mathrm{l} / \mathrm{h})$ and a mean flow speed of the salt buffer solution $([\mathrm{KCl}]=200 \mathrm{mM})$ in the inlet of $310 \mu \mathrm{m} / \mathrm{s}(80 \mu \mathrm{l} / \mathrm{h})$ sampled with a $20 \mu \mathrm{m} \times 5 \mu \mathrm{m}(x \times y)$ spot at $y=0$. (a) Mean (circles), minimum (upwards triangles) and maximum (downward triangles) salt concentrations sampled for different locations along the flow. The color code corresponds to the one in chapter 7 , the error of the mean is smaller than the symbols. The salt concentration approaches $160 \mathrm{mM}$ and its distribution narrows. (b) Mean (circles), minimum (upwards triangles) and maximum (downward triangles) vimentin assembly times sampled for different locations along the flow. The color code corresponds to the one in section 6.2 and chapter 7 , see figure $6.18 \mathrm{a}$, the error of the mean is again smaller than the symbols. From abut $300 \mu \mathrm{m}$ downstream the increase of all three values is mostly linear and at low $x$ the maximum value grows very fast. 
time at $x=0$ is $875 \mathrm{~ms}$ and increases by a factor of ten to $7.85 \mathrm{~s}$ over the next millimeter. On the same range the mean assembly time increases from $8 \mathrm{~s}$ to $20 \mathrm{~s}$. Again, the standard deviation is surprisingly low, below $10 \mathrm{~ms}$.

We see that by either just looking at the mid-plane $(z=0)$ or by just considering the means instead of the complete set of assembly time and salt concentration combinations in the probe volume we loose valuable information.

Now that we understand the flow for one set of conditions we can investigate how changing these conditions affects the flow and the resulting ensemble of environments for the vimentin.

\section{Comparison of different geometries}

First of all, we check how the reduced height of the protein inlet affects the flow. Figure 6.5 shows comparisons of the protein and salt concentration distributions for devices with reduced protein inlet height and without this feature. The flow speed in the protein inlet is equal for both cases which results in an about 2.2 times larger flow rate for the larger inlet. As a direct consequence, the hydrodynamic focusing by the salt buffer is weaker and the final protein jet is broader (figure 6.5(a) and (c)). This implies that the distance the salt has to diffuse for complete mixing increases. As the overall flow speed also increases, the diffusion of salt into the protein jet in the simulated volume is considerably reduced (figure 6.5(b)). When we compare the mid $y-z$ planes of the intersection we additionally see that the shape of the vimentin jet is drastically different $(x=0)$ : an ogival shape which does not touch the bottom and top of the device for the reduced protein inlet height and a homogeneous rectangle for equal inlet height. This shape changes are also reflected in the salt concentration. The increased diffusion of salt towards the top and bottom of the device is due to the lower flow speeds in these regions.

Next, we compare how these changes in the solute distributions affect the ensemble of salt concentrations and assembly times. For the salt concentration, we see that as we would expect from the above, the mean and minimum concentrations raise more slowly. In the middle of the intersection, the salt concentration still is below the minimum concentration at this location for the device with reduced protein inlet height, which is about $5 \mathrm{mM}$. It remains below that minimum value and reaches about $95 \mathrm{mM}$ at $1 \mathrm{~mm}$ further downstream. Similarly, the minimum concentration rises from $0 \mathrm{mM}$ to $87 \mathrm{mM}$ on the same distance for the device with equal channel height. For this device, the maximum salt concentration in the middle of the intersection is $12 \mathrm{mM}$, i.e. between the minimum and the mean of the flow with reduced 


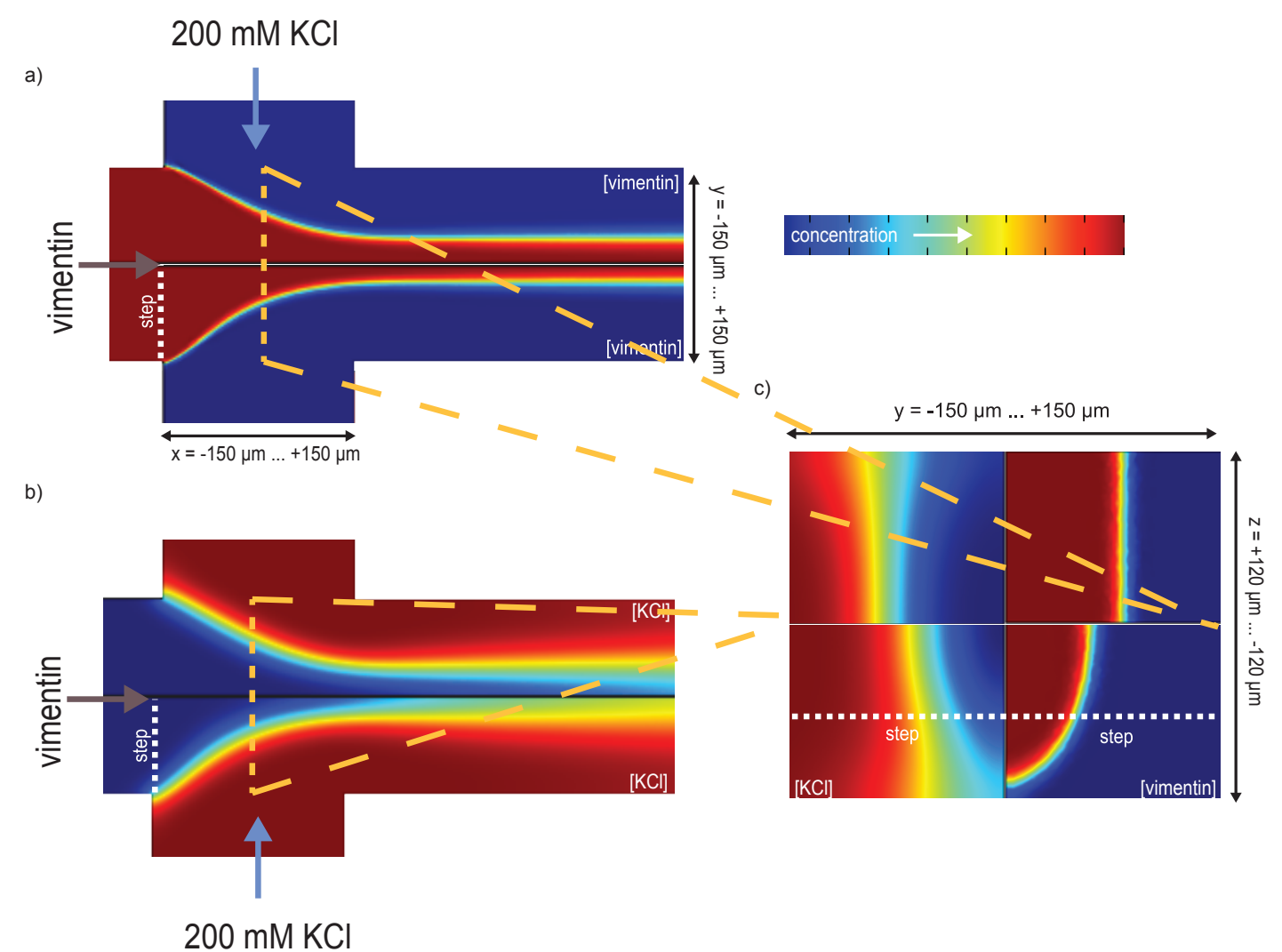

Figure 6.5: Results of the simulation of vimentin flow in microfluidic devices with a reduced height of the vimentin inlet (bottom part of the subfigures) and without it (top part of the subfigures). The "step" line marks the location of the change in channel height. The mean flow speed of the vimentin solution $\left(c_{v i m}=4 \mathrm{mg} / \mathrm{ml}\right)$ in the inlet is $335 \mu \mathrm{m} / \mathrm{s}$ (40 $\mu \mathrm{l} / \mathrm{h}$ with the reduced inlet size, $87 \mu \mathrm{l} / \mathrm{h}$ without it) and the mean flow speed of the salt buffer solution in the inlet is $310 \mu \mathrm{m} / \mathrm{s}(80 \mu \mathrm{l} / \mathrm{h})$. (a) Comparison of the vimentin concentration in the mid $x-y$ plane $(z=0)$ of the device for both geometries. For the case of constant channel height, the vimentin jet is slightly larger, due to the increased flow rate. (b) Comparison of the salt concentration for the same plane. Because of the reduced hydrodynamic focusing and increased overall flow speed, the increase of salt concentration along the mid- $x$-axis is reduced for the case of constant channel height. (c) Comparison of the vimentin (right part) and potassium chloride (left part) concentrations in the mid $y-z$ plane of the intersection $(x=0)$ for both geometries. Most noticeably, the vimentin jet has equal width from bottom to top for the constant height case as opposed to the more ogival form observed for the reduced inlet height. This is also reflected in the salt concentration distribution. It is also clearly visible that the hydrodynamic focusing is weaker. 
(a)

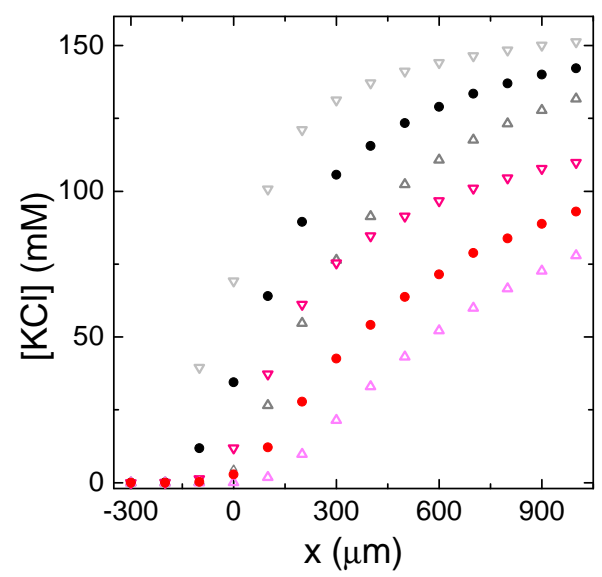

(b)

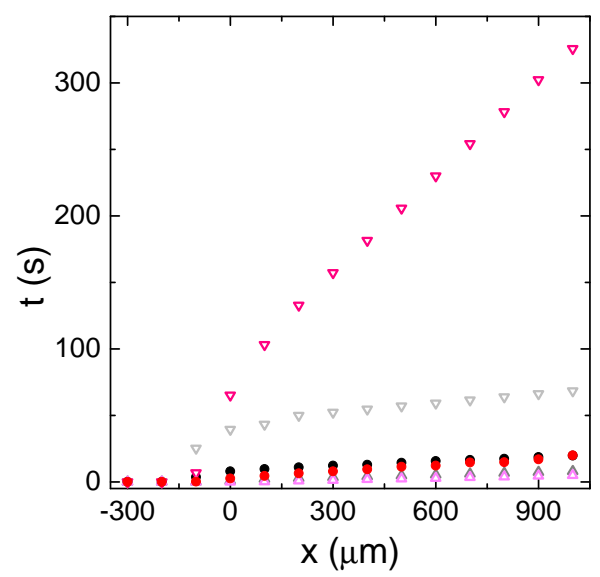

Figure 6.6: Comparison of the mean (circles), minimum (upwards triangles) and maximum (downward triangles) values of (a) the salt concentrations and (b) assembly times found in the probe volume for different locations in the flow for devices with reduced protein inlet height (black and gray) and equal overall device height (red and magenta). The mean inlet flow speeds for both cases are set equal to $310 \mu \mathrm{m} / \mathrm{s}(80 \mu \mathrm{l} / \mathrm{h})$ for the buffer and $335 \mu \mathrm{m} / \mathrm{s}(40 \mu \mathrm{l} / \mathrm{h}$ with the reduced inlet size, $87 \mu \mathrm{l} / \mathrm{h}$ without it) for the protein. At equal channel height, the salt concentration rises more slowly and to a lower level. The mean and minimum assembly times are very similar, but for equal channel height the maximum assembly time increases much faster. 
inlet size. Already $300 \mu \mathrm{m}$ further downstream, it is below that minimum value and reaches only $110 \mathrm{mM}$ at $1 \mathrm{~mm}$ below the intersection. The lower salt concentrations further downstream are due to the fact that the increased protein flow rate reduces the mean salt concentration in the flow to $130 \mathrm{mM}$. The maximum and minimum require a larger distance to converge to each other which is due to the increase of the distance that needs to be covered by diffusion and the increased overall flow speed, resulting in an overall increased Péclet number.

In contrast, the mean and minimum assembly times are very similar for both cases. The mean increases slightly faster for equal channel height reaching identical values after $1 \mathrm{~mm}$ despite lower early assembly times. However, the maximum assembly time increases much faster at equal channel height reaching over 5 min instead of just $1 \mathrm{~min}$. The reason for the existence of these very long assembly times, which are also to some extent reflected in the mean, is the existence of protein flow at very low speed close to the top and bottom. Therefore, the reduced channel height for the inlet not only prevents adhesion of the protein to the channel wall but also narrows the distribution of assembly times. To even better understand the changes due to

(a)

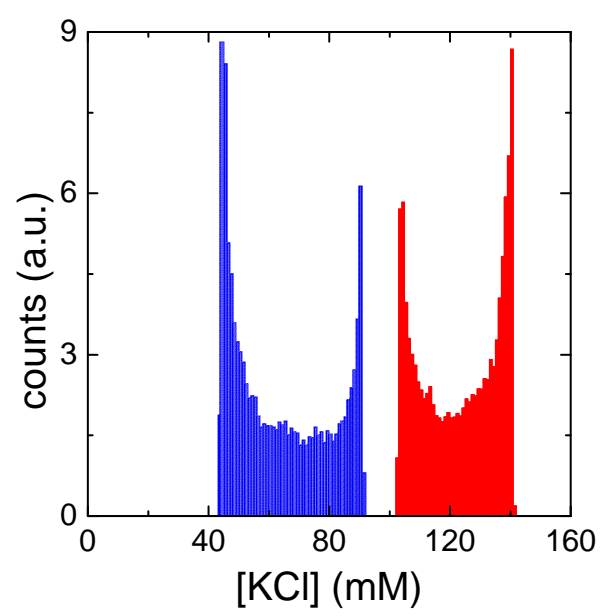

(b)

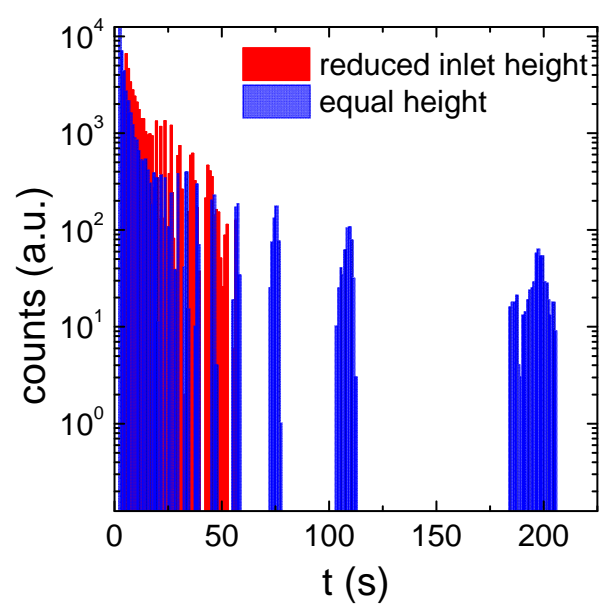

Figure 6.7: Comparison of the histograms of (a) the salt concentrations and (b) assembly times found in the probe volume found at $x=500 \mu \mathrm{m}$ for devices with reduced protein inlet height (red) and equal overall device height (blue). The mean inlet flow speeds for both cases are set equal to $310 \mu \mathrm{m} / \mathrm{s}(80 \mu \mathrm{l} / \mathrm{h})$ for the buffer and $335 \mu \mathrm{m} / \mathrm{s}(40 \mu \mathrm{l} / \mathrm{h}$ with the reduced inlet size, $87 \mu \mathrm{l} / \mathrm{h}$ without it) for the protein. At equal channel height, relatively lower salt concentrations are more common. The histogram for the assembly times initially decays faster but shows contributions up to $200 \mathrm{~s}$.

the differences in the protein inlet height we also compare the histograms for the salt concentrations and assembly times at $x=500 \mu \mathrm{m}$ (figure 6.7). The histograms 
for the salt concentrations appear mirrored: For overall equal channel height, values closer to the minimum are more common than those closer to the maximum whereas for the reduced protein inlet height the opposite is the case. This shift might be due to the fact that in the equal height geometry the contact area between the protein jet and the salt buffer (and especially the part of it probed by the X-ray beam), which is the main contributor to higher salt concentrations, is reduced (see figure 6.5). Simultaneously, the rectangular form of the protein jet results in a more homogeneous diffusion which increases the contribution at lower salt concentrations. When we regard the histograms for the assembly times we see that the initial decay at low assembly times is stronger in the case of equal channel height than it is for the case of reduced protein inlet height. However, whereas the latter case has no contributions above $75 \mathrm{~s}$, at equal height some vimentin reaches assembly times of over 200 s, i.e. it flows significantly slower than the majority. This is obviously due to vimentin very close to the channel walls where the flow speed is close to 0 . As this portion is very small, it barely affects the mean assembly time as we saw in figure $6.6 \mathrm{~b}$.

\section{Effect of flow speed on the mixing}

The conditions of flow in a microfluidic device can be easily altered by changing the flow speeds. Firstly, we consider how changes in the absolute flow speed at constant buffer to protein solution flow speed ratio alter the salt concentrations and assembly times.

In this case, the size of the protein jet remains constant, and changes in the diffusion of salt are due to changes in the Péclet number. As an example we compare the case where buffer inlet flow speeds are $620 \mu \mathrm{m} / \mathrm{s}(160 \mu \mathrm{l} / \mathrm{h})$ and the protein flow speed is $670 \mu \mathrm{m} / \mathrm{s}(80 \mu \mathrm{l} / \mathrm{h})$ ("fast flow") and the case where buffer inlet flow speeds are $77.5 \mu \mathrm{m} / \mathrm{s}(20 \mu \mathrm{l} / \mathrm{h})$ and the protein flow speed is $83.75 \mu \mathrm{m} / \mathrm{s}(10 \mu \mathrm{l} / \mathrm{h})$ ("slow flow"), see figure 6.8. In the "slow flow" case, the mean salt concentration is slightly above 0 already $50 \mu \mathrm{m}$ before the beginning of the intersection, i.e. inside the protein inlet. In the middle of the intersection it already succeeds half of the equilibrium value and $1 \mathrm{~mm}$ further downstream, even the minimum salt concentration in the probe volume reaches the equilibrium value. In contrast to that, in the "fast flow" case, the minimum salt concentration is still 0 in the middle of the intersection, whereas the maximum value $(58 \mathrm{mM})$ is below the minimum of the "slow flow" case. At $1 \mathrm{~mm}$ downstream, the salt concentration does not exceed $145 \mathrm{mM}$ with a mean of $125 \mathrm{mM}$. 
(a)

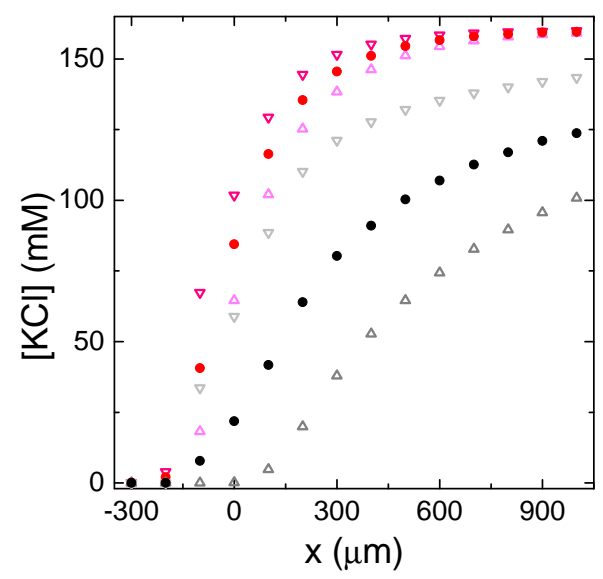

(b)

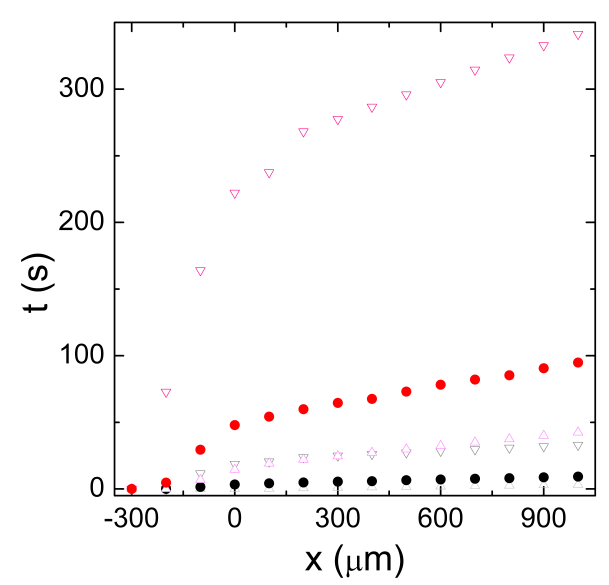

Figure 6.8: Comparison of the mean (dots), minimum (upwards triangles) and maximum (downward triangles) values of (a) the salt concentrations and (b) and assembly times found in the probe volume for different locations in the flow for buffer inlet flow speeds of $620 \mu \mathrm{m} / \mathrm{s}(160 \mu \mathrm{l} / \mathrm{h})$ and protein flow speeds of $670 \mu \mathrm{m} / \mathrm{s}(80 \mu \mathrm{l} / \mathrm{h})$ (black and gray), respectively , $77.5 \mu \mathrm{m} / \mathrm{s}(20 \mu \mathrm{l} / \mathrm{h})$ and protein flow speeds of $83.75 \mu \mathrm{m} / \mathrm{s}(10 \mu \mathrm{l} / \mathrm{h})($ red and magenta), i.e. for different absolute flow speeds but identical flow speed ratios. At the lower flow speed, the concentration is equilibrated to $160 \mathrm{mM}$ at $1 \mathrm{~mm}$ from the intersection, whereas at the higher flow speed it is still rather broad. The reduced flow speed directly leads to longer assembly times for the same location in the flow and their distribution is rather broad. 


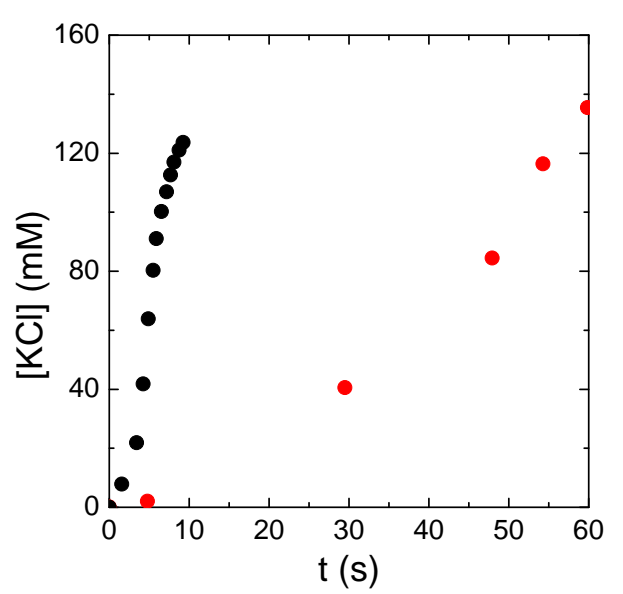

Figure 6.9: The combination of mean salt concentrations and mean assembly times for each measurement point depends on the flow speed. The figure shows the correlation between the mean salt concentration and the mean assembly time for buffer inlet flow speeds of $620 \mu \mathrm{m} / \mathrm{s}(160 \mu \mathrm{l} / \mathrm{h})$ and protein flow speeds of $670 \mu \mathrm{m} / \mathrm{s}(80 \mu \mathrm{l} / \mathrm{h})$ (black), respectively, $77.5 \mu \mathrm{m} / \mathrm{s}(20 \mu \mathrm{l} / \mathrm{h})$ and protein flow speeds of $83.75 \mu \mathrm{m} / \mathrm{s}$ $(10 \mu \mathrm{l} / \mathrm{h})(\mathrm{red})$.

ent.

Figure 6.9 shows how the combinations of the mean salt concentrations and mean assembly times differ for the two different overall flow speeds. We see that in both cases the mean salt concentration increases with the assembly time. However, despite the fact that the mixing length for the lower flow speed is shorter, the mean assembly time for each salt concentration is actually longer for the lower flow speed, i.e. mixing is slower. For example, the assembly time which corresponds to a salt concentration of $100 \mathrm{mM} \mathrm{KCl}$ distinguishes by nearly a factor of ten. This insight will become important when attempting to distinguish assembly time and salt concentration effects in section 7.3. Additionally, figure 6.9 illustrates that due to the non-linearity of the assembly time dependence of the probe location in the intersection area (see figure $6.8 \mathrm{~b}$ ) the temporal resolution for lower flow speeds worsens considerably to the range of several seconds. 
Another possibility to alter the assembly time salt concentration ensembles while maintaining the device geometry and inlet salt concentrations, is to change the relative flow speeds. To understand how such changes affect the assembly conditions we compare the salt concentrations and assembly times for flow with identical protein flow rates but different buffer flow rates. Decreasing the buffer to protein flow rate

(a)

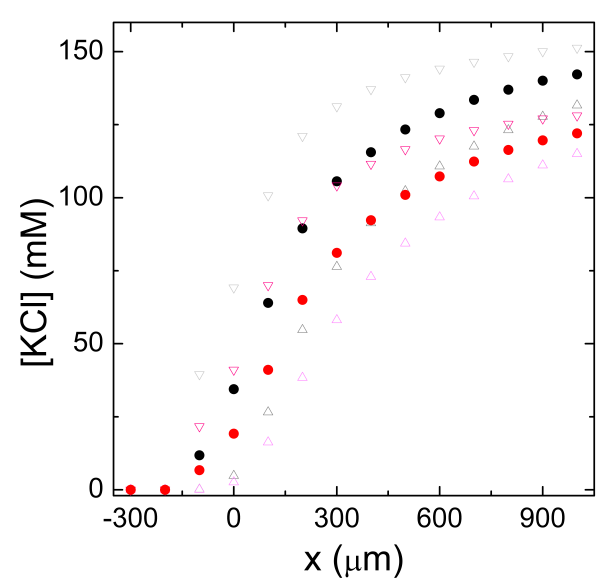

(b)

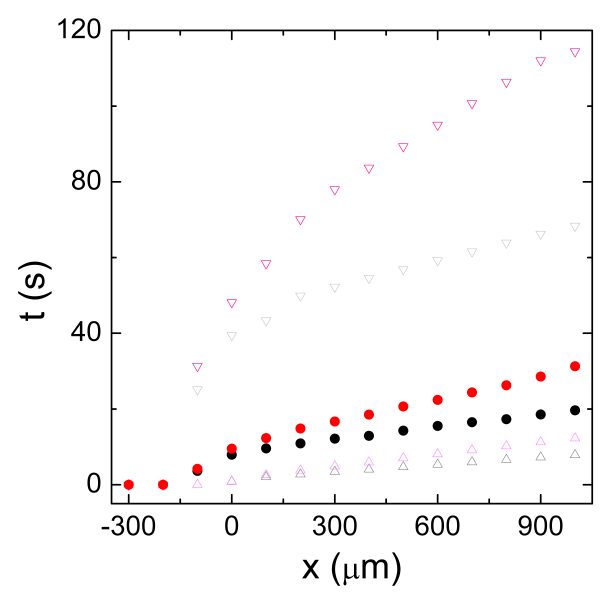

Figure 6.10: Comparison of the mean (circles), minimum (upwards triangles) and maximum (downward triangles) values of (a) the salt concentrations and (b) and assembly times found in the probe volume for different locations in the flow for identical protein flow speeds of $335 \mu \mathrm{m} / \mathrm{s}(40 \mu \mathrm{l} / \mathrm{h})$ and different buffer inlet flow speeds: $310 \mu \mathrm{m} / \mathrm{s}(80 \mu \mathrm{l} / \mathrm{h})$ (black and gray) and $155 \mu \mathrm{m} / \mathrm{s}(40 \mu \mathrm{l} / \mathrm{h}$ )(red and magenta). The increased thickness of the protein jet (due to less focusing) at the lower buffer flow speed and the lower mean salt concentration result in overall lower values for the salt concentration distribution. The curves for the assembly times are nearly parallel with values for the reduced buffer speed about a factor of 1.5 higher.

reduces the hydrodynamic focusing and increases the size of the vimentin jet. At fixed protein flow rate it also decreases the overall flow rate. When comparing the salt concentrations, we see that for the lower ratio mean, maximum and minimum values are lower (figure 6.10a). This has two main reasons: For low $x$, the increased size of the protein jet slows down diffusion and for higher $x$ the decreased equilibrium salt concentration puts an upper limit for all three values. Also, the maximum and minimum values come closer together within the simulation volume as the overall flow speed is decreased. When we compare the assembly times we find that despite the reduced salt concentrations at the beginning of the intersection, all three curves have a similar form in both cases. For the reduced buffer inlet speed, the assembly times are about a factor 1.5 higher which is mostly due to the decrease of the overall 
flow speed by 0.6 of the original value.

Changing the relative flow speeds provides a tool to change equilibrium salt concentration in the device and it is especially useful for direct comparisons of "long time" changes at different salt concentrations, as corresponding positions in the flow can be easily estimated.

\section{Effect of buffer salt concentration}

Additionally, we ask how far changing the salt concentration in the buffer affects the salt distribution and the assembly times. As the viscosity of the protein jet depends on the salt concentration, this is not completely obvious. However, as the changes in viscosity are only small, we expect no large effect. In figure 6.11 we compare

(a)

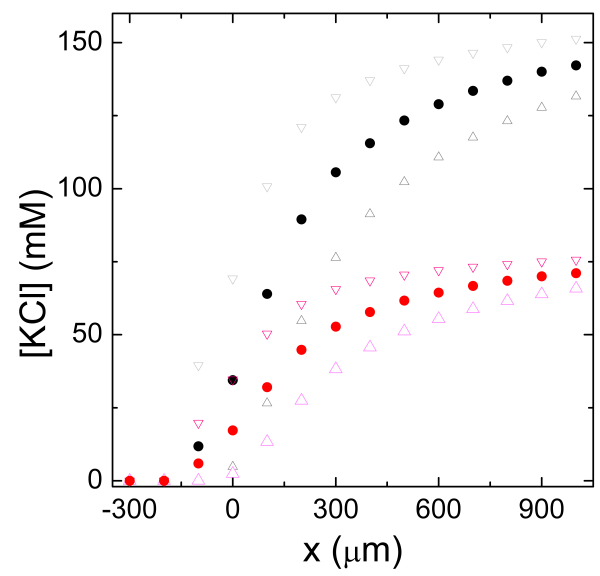

(b)

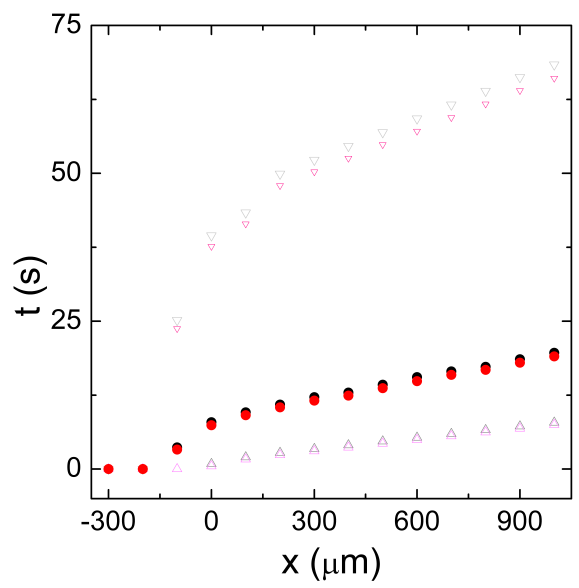

Figure 6.11: Comparison of the mean (circles), minimum (upwards triangles) and maximum (downward triangles) values of (a) the salt concentrations and (b) assembly times found in the probe volume for different locations in the flow for buffers salt concentrations of $200 \mathrm{mM} \mathrm{KCl}$ (black and gray) and $100 \mathrm{mM} \mathrm{KCl}$ (red and magenta). We see that the salt concentrations scale with a factor of two and that the assembly times are only slightly smaller for the lower salt concentrations.

the salt concentrations and assembly times for buffer salt concentrations of $100 \mathrm{mM}$ $\mathrm{KCl}$ and $200 \mathrm{mM} \mathrm{KCl}$, at otherwise identical conditions. As we would expect, the salt concentrations scale nearly perfectly with a factor of two, i.e. any difference induced by the change in viscosity is minor. The assembly times differ less than $1 \mathrm{~s}$, with the lower salt assembly times being lower as the minimal salt concentration for integration of the assembly time is reached later. This implies that by changing 
the buffer salt concentration we can directly compare the protein state at identical assembly times but different salt concentrations.

\section{Effect of size of the probe volume}

Finally, we ask how changes in the probe volume affect the ensemble of salt concentrations of assembly time. Therefore, we compare two more "extreme" cases: A $5 \mu \mathrm{m} \times 5 \mu \mathrm{m}$ X-ray spot as it is available at ESRF ID13 and a $50 \mu \mathrm{m} \times 50 \mu \mathrm{m}$ spot as it is available at ESRF ID02, i.e. probe volumes that differ in two orders of magnitude. This allows us to estimate how critical it is to know the correct size of the X-ray beam. Figure 6.12 shows that the maximum concentrations for the larger

(a)

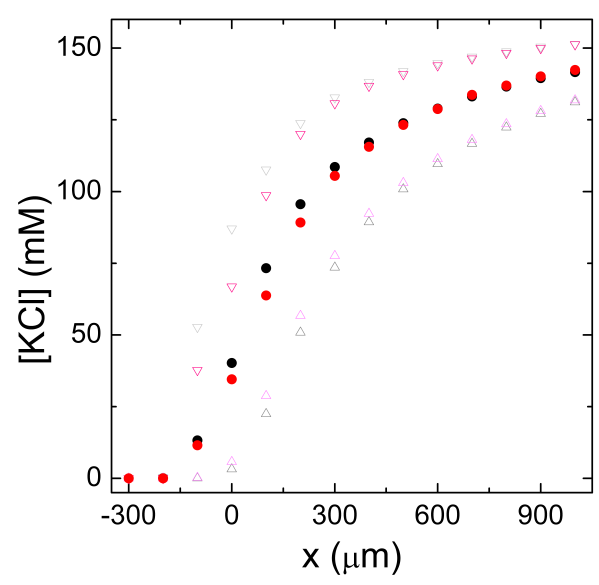

(b)

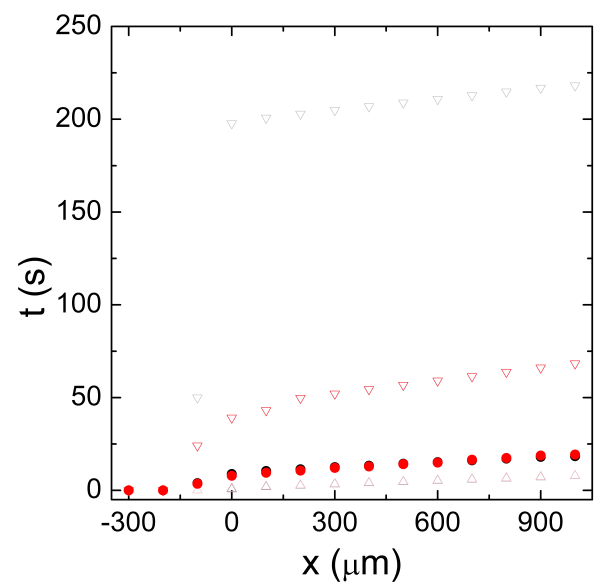

Figure 6.12: Comparison of the mean (circles), minimum (upwards triangles) and maximum (downward triangles) values of (a) the salt concentrations and (b) assembly times found in the probe volume for different locations in the flow for a $50 \mu \mathrm{m} \times 50 \mu \mathrm{m}$ probe (black and gray) and $5 \mu \mathrm{m} \times 5 \mu \mathrm{m}$ (red and magenta). The larger spot size increases the maximum concentration and reduces the minimum concentration, the mean increases. For the assembly times the minimum and mean values remain similar, but the maximum increases by a factor of about 3.5.

spot are always larger and the minimum salt concentrations are always smaller than for the smaller spot. the mean salt concentrations tend to be larger, with the biggest difference of $10 \mathrm{mM}$ at $x=100 \mu \mathrm{m}$. At $1 \mathrm{~mm}$ downstream from the intersection, the mixing has proceeded far enough for the differences to diminish.

When we consider the assembly times, we see little difference in the mean and minimum values, but the maximum values increase by about a factor of 3.5, i.e. a smaller 
spot size is instrumental to avoiding long assembly time components in the signal.

\section{Effect of pre-focusing}

In section 5.2 we introduced the idea of decreasing the mixing times by adding a prefocusing buffer flow (see figure 5.2). In our experiments we simultaneously reduced the channel width to $100 \mu \mathrm{m}$, which also decreases the width of the protein jet and thereby the mixing time. This leads to changes in the manufacturing protocol and the height of the protein inlet was increased to about $130 \mu \mathrm{m}$ (instead of previously $110 \mu \mathrm{m}$ ) at a constant overall channel height of $240 \mu \mathrm{m}$. We now aim to understand how these changes affect the distribution of solutes and the assembly times.

Figure 6.13 shows a comparison of the vimentin and salt concentration distributions in the pre-focusing devices and the previously discussed devices at similar flow rates, which due to the changes in the channel width implies that in the pre-focusing case the flow speeds are about a factor of three larger. We see that in the middle of the protein inlet - salt inlet intersection (i.e. at $x=0$ ) the focusing in the pre-focusing geometry is already further progressed than in the simple intersection case. The focusing also appears tighter which is most likely due not only to the change in geometry but also to the increase in the total buffer flow rate (i.e. salt buffer and salt free buffer). Due to the reduced width of the protein jet, the diffusion is much more visible. When we consider the salt concentration distributions we see that the pre-focusing flow changes its shape drastically. As the salt free buffer has the lowest flow rate in the system, the salt actually extends towards the buffer inlet resulting in a gradient whose isolines are nearly parallel to the main flow direction. We can also see that the salt free buffer indeed focuses the protein jet before its first contact with salt. Further downstream we see that despite very similar Péclet numbers (assuming that the characteristic length scale corresponds the channel width $w$, $\mathrm{Pe}=\frac{U w}{D} \frac{Q}{h D}$ where $Q=U w h$ is the typical flow rate and $h$ the channel height) the gradient is further diminished for the pre-focusing case which is most likely due to the improved focusing. When we consider the $y$-z-plane for $x=0$, we see that the shape of both distributions is rather similar in both geometries. Unexpectedly, though, the vimentin jet is further removed from the channel walls in the pre-focusing case despite an increased inlet height. This is most likely due to the increase of total buffer flow due to the addition of the salt free buffer flow.

For a better understanding of the changes in the mixing we again consider the mean, minimum and maximum salt concentrations and assembly times for different 


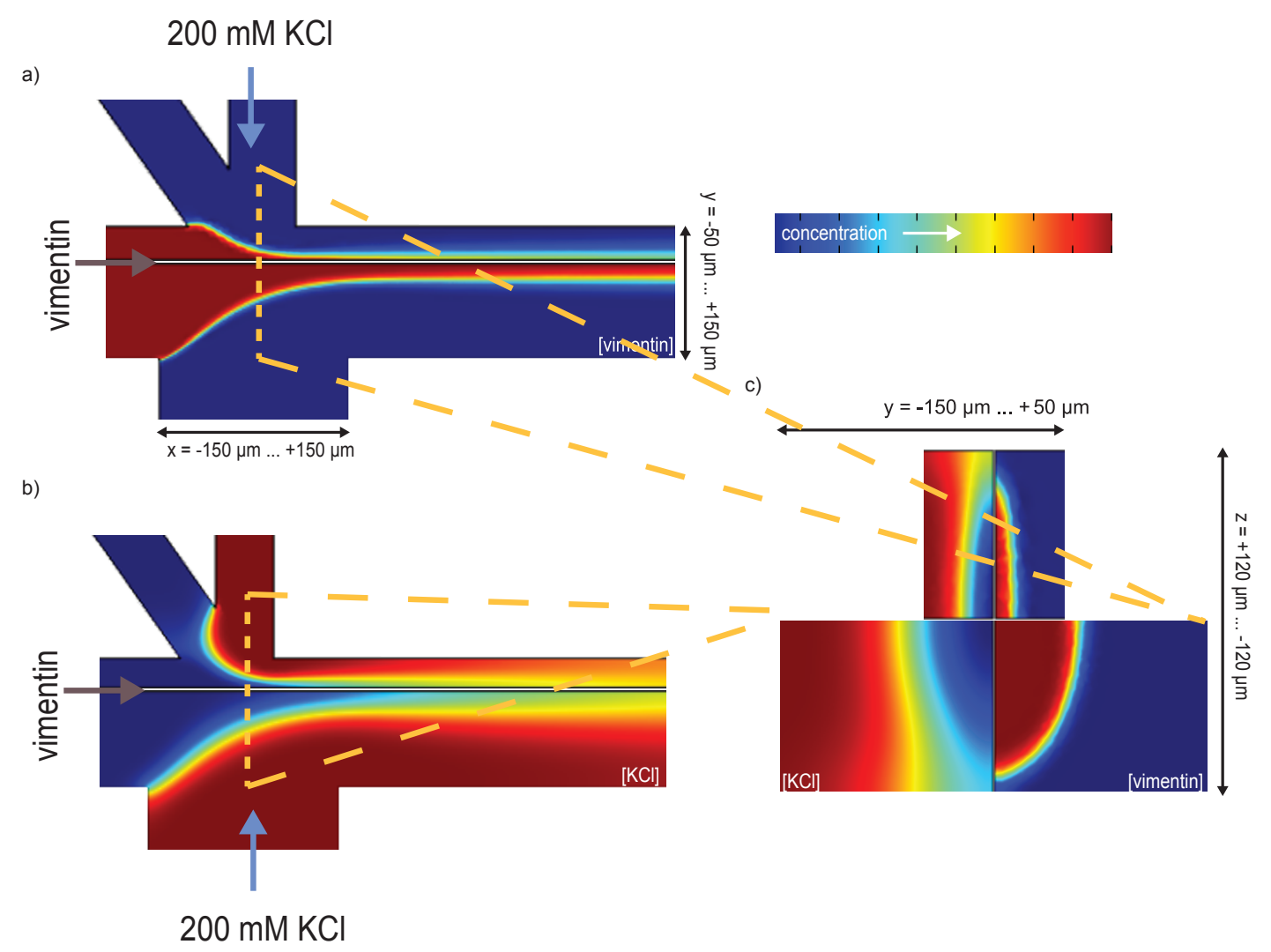

Figure 6.13: Results of the simulation of vimentin flow in microfluidic devices with a channel width of $300 \mu \mathrm{m}$ and of $100 \mu \mathrm{m}$, respectively. For the thinner channels the vimentin is pre-focused by buffer at a $45^{\circ}$ angle. The mean flow speed of the vimentin solution $([$ vimentin] $=4 \mathrm{mg} / \mathrm{ml})$ in the inlet is $335 \mu \mathrm{m} / \mathrm{s}$, respectively $850 \mu \mathrm{m} / \mathrm{s}(40 \mu \mathrm{l} / \mathrm{h})$, the mean flow speed of the salt buffer solution $(200 \mathrm{mM}$ and $85 \mathrm{mM}$ respectively) in the inlet is $310 \mu \mathrm{m} / \mathrm{s}$, respectively $930 \mu \mathrm{m} / \mathrm{s},(80 \mu \mathrm{l} / \mathrm{h})$ and the flow speed of the salt free buffer is $58 \mu \mathrm{m} / \mathrm{s}(5 \mu \mathrm{l} / \mathrm{h})$. (a) Comparison of the vimentin concentration in the mid $x-y$ plane $(z$ $=0$ ) of the device for both geometries. The addition of pre-focusing flow results in tighter focusing and the overall reduced size increases the effect of diffusion. (b) Comparison of the salt concentration for the same plane. Despite an increased overall flow speed in the pre-focusing geometry, the salt gradient equilibrates closer to the intersection. (c) Comparison of the vimentin (right part) and potassium chloride (left part) concentrations in the mid $y$ - $z$ plane of the intersection $(x=0)$ for both geometries. The overall shape is quite similar for both cases. Noticeably, in the pre-focusing case the vimentin jet is further removed from the top and bottom of the device despite a slightly increased inlet size. 
(a)

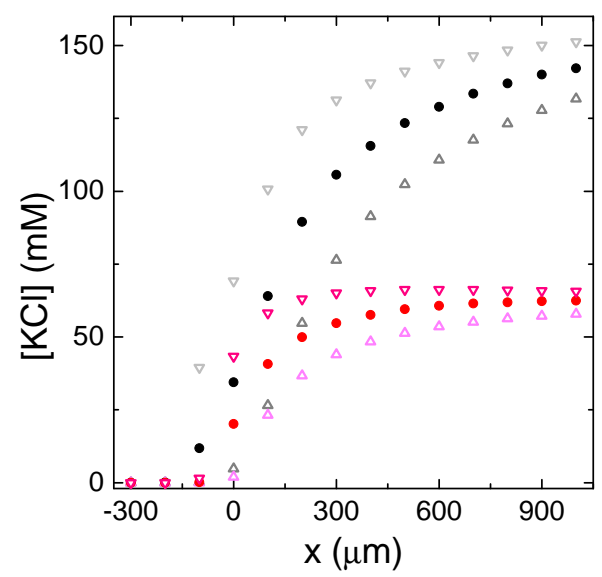

(b)

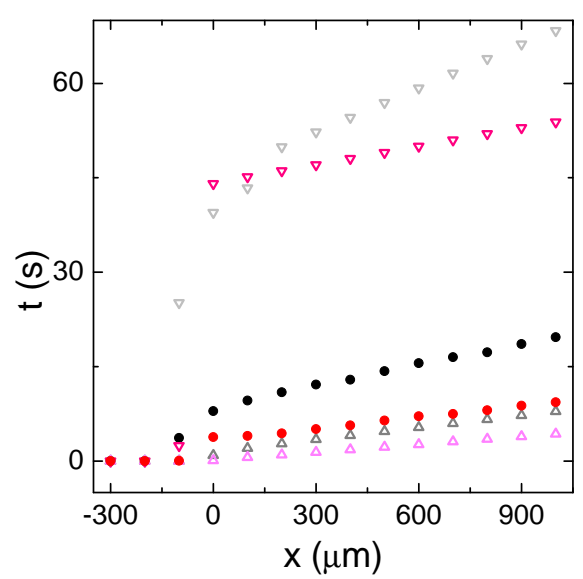

Figure 6.14: Comparison of the mean (dots), minimum (upwards triangles) and maximum (downward triangles) values of (a) the salt concentrations and (b) assembly times found in the probe volume for different locations in the flow for a simple cross geometry with a channel width of $300 \mu \mathrm{m}$ and a buffer salt concentration of $200 \mathrm{mM}$ (black and gray) and the pre-focusing geometry with a channel width of $100 \mu \mathrm{m}$ and a buffer salt concentration of $85 \mathrm{mM}$ (red and magenta) at equal flow rates (vimentin $40 \mu \mathrm{l} / \mathrm{h}$, salt buffer $80 \mu \mathrm{l} / \mathrm{h}$, salt free buffer $5 \mu \mathrm{l} / \mathrm{h}$ ). The salt concentration equilibrates at much shorter distances in the case of pre-focusing and the mean assembly times are about a factor of two lower. 
locations in the device, see figure 6.14. For the salt concentrations, we see that the maximum and minimum curves for the pre-focusing case converge on much shorter length scales, and the increase takes place on a shorter length scale (about $225 \mu \mathrm{m}$ as opposed to about $485 \mu \mathrm{m}$ from a simple exponential decay fit). The form of the assembly time curves is much more similar, although in the pre-focusing case the curves become linear on shorter timescales which could be expected because of the reduced channel width. Because of the increased flow speed, the mean assembly times are shorter by a factor of about two which in combination with the reduced mixing length results in a reduction of the mixing time of about a factor of four.

Finally, we compare the histograms of salt concentrations and assembly time for

(a)

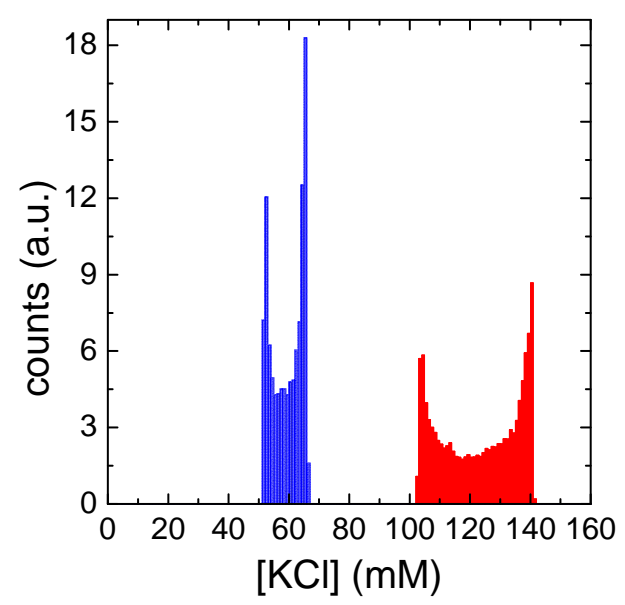

(b)

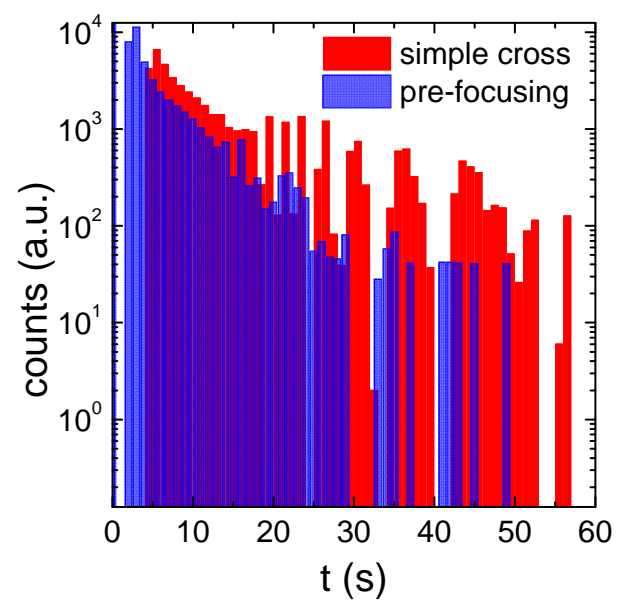

Figure 6.15: Comparison of the histograms of (a) the salt concentrations and (b) assembly times found in the probe volume at $x=500 \mu \mathrm{m}$ in the flow for a simple cross geometry with a channel width of $300 \mu \mathrm{m}$ and a buffer salt concentration of $200 \mathrm{mM}$ (red) and pre-focusing geometry with a channel width of $100 \mu \mathrm{m}$ and a buffer salt concentration of $85 \mathrm{mM}$ (blue) at equal flow rates (vimentin $40 \mu \mathrm{l} / \mathrm{h}$, salt buffer $80 \mu \mathrm{l} / \mathrm{h}$, salt free buffer $5 \mu \mathrm{l} / \mathrm{h}$ ). In the pre-focusing geometry the salt concentration histogram is centered at lower concentrations due to the reduced buffer salt concentration and is more compact but its general form resembles the one observed for the simple cross. The assembly time histogram is shifted towards shorter times.

fixed positions in both geometries (see figure 6.15). At $x=500 \mu \mathrm{m}$ the most obvious difference in the salt concentration histogram is that for the pre-focusing geometry it is centered at smaller concentrations. As the salt concentration in the buffer solution is reduced from $200 \mathrm{mM}$ to $85 \mathrm{mM}$ this is not unexpected. The shape of both histograms is quite similar (a U-shape with higher values at the upper end) but in the pre-focusing case the distribution is narrower by a factor of nearly three. 
The assembly time histogram for the pre-focusing geometry is shifted to smaller assembly times and the amount of vimentin at higher assembly times is strongly reduced due to the larger distance of the vimentin jet to all channel walls.

In summary, the changes in the device layout result in shorter assembly times at identical probing location and narrow both the ensemble of assembly times and the ensemble of salt concentrations resulting in a better defined state of the vimentin in the probe volume.

\subsubsection{Extrapolating beyond the simulation volume}

In order to access longer assembly times, we need to probe the microflow outside the simulation volume $(x>1 \mathrm{~mm})$. In principle, extending the simulation volume would provide the relevant information, but the large difference between the channel cross-section (less than $1 \mathrm{~mm}$ ) and length (up to more than $100 \mathrm{~mm}$ ) renders this approach unfeasible. Therefore, estimating the ensemble conditions of vimentin at points further downstream requires extrapolation of the simulation results. However, we can solve this problem by extrapolating individual streamlines and concentrations, velocities and assembly times along the streamlines. The most relevant values are the $y$-coordinate to know whether or not a point is part of the probe volume, the salt concentration, the protein concentration and the assembly time. Figures 6.16 and 6.17 show these values for the streamline starting as $y=75 \mu \mathrm{m}$ and $z=27 \mu \mathrm{m}$ (i.e. in the middle of the simulated quadrant). The $y$ - and $z$-components of the velocity field in this region are very close to zero and can be neglected. The $x$ component (i.e. $u$ ) is nearly constant and in direct consequence the assembly time increases linearly. Therefore we extrapolate $u$ with its mean between $x=0.5 \mathrm{~mm}$ and $x=0.75 \mathrm{~mm}$ and the assembly time with the linear function corresponding to the assembly time at these two points. This range of points was selected in order to avoid artifacts from the outlet (see figure 6.16b). The $y$-coordinate is constant above $250 \mu \mathrm{m}$ and can also be extrapolated by its mean between $x=0.5 \mathrm{~mm}$ and $x=0.75 \mathrm{~mm}$. The salt concentration can be described with a double-exponential fit decay to the final mean concentration between $x=150 \mu \mathrm{m}$ and $x=1 \mathrm{~mm}$. Finally, the simulation overestimates the diffusion of vimentin resulting in a decay in the concentration along the streamline. We assume that the concentration of vimentin remains mostly constant after the hydrodynamic focusing and choose the concentration at $x=150 \mu \mathrm{m}$ as fixed value for the extrapolation. 
(a)

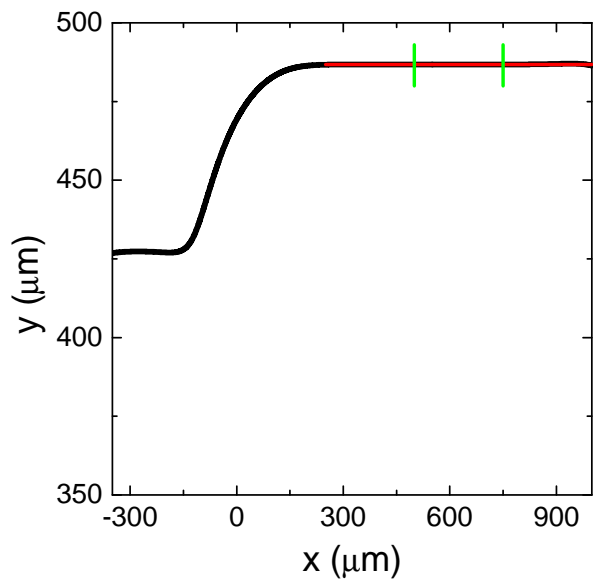

(b)

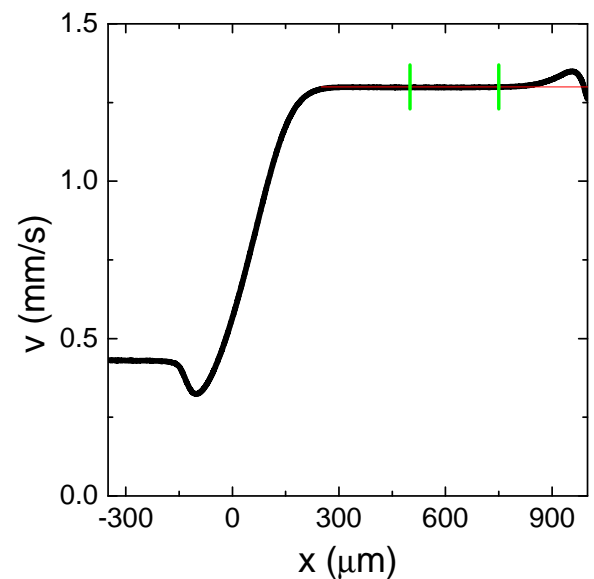

Figure 6.16: Examples of simulation results (black lines) along a single streamline and their extrapolation functions to larger $x$ (red lines) for the streamline starting at $y=$ $75 \mu \mathrm{m}$ and $z=27 \mu \mathrm{m}$. The green lines denote the $x$-range used for determining the extrapolation function. (a) The $y$-position and (b) the velocity are approximated by constant values based on their means on a fixed $x$-range. See also figure 6.17.

\subsection{Predicting SAXS curves from the simulations}

The next question we can now address is how much the different flow conditions alter the SAXS curves and, reversely, how high the information content of the measured SAXS curves is.

\subsubsection{Instantaneous filament formation}

There are several levels of complexity for modeling the SAXS curves. The most basic approach is to assume that vimentin at a certain salt concentration instantaneously forms filaments which correspond to the equilibrium state at this concentration. As we know the scattering signal of vimentin at six different potassium chloride concentrations between $0 \mathrm{mM}$ and $100 \mathrm{mM}$ (see chapter 4 ) we interpolate $I\left(q, c_{s}\right)$ for each $q$ linearly between those for concentrations below $100 \mathrm{mM}$ and extrapolate linearly to higher concentrations based on the changes between $80 \mathrm{mM}$ and $100 \mathrm{mM}$. We then calculate the SAXS curve for each probe spot via equation 6.3 based on our knowledge of the salt concentration ensemble at each location.

Figure 6.18 shows the results of this estimation for some exemplary points. We see that before the intersection (red dots) the scattering signal corresponds to tetrameric 
(a)

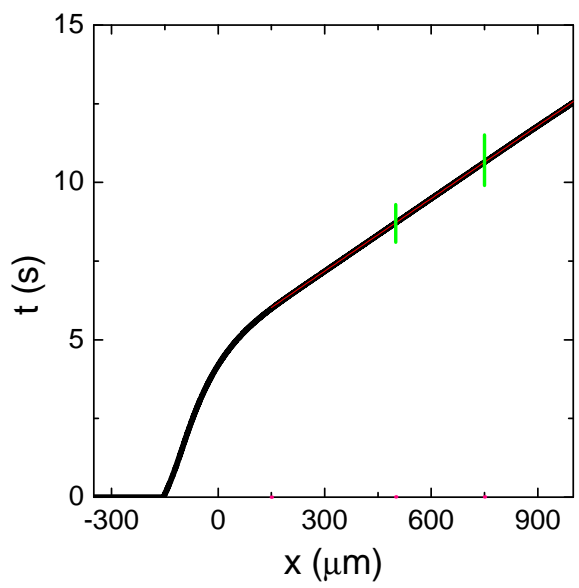

(b)

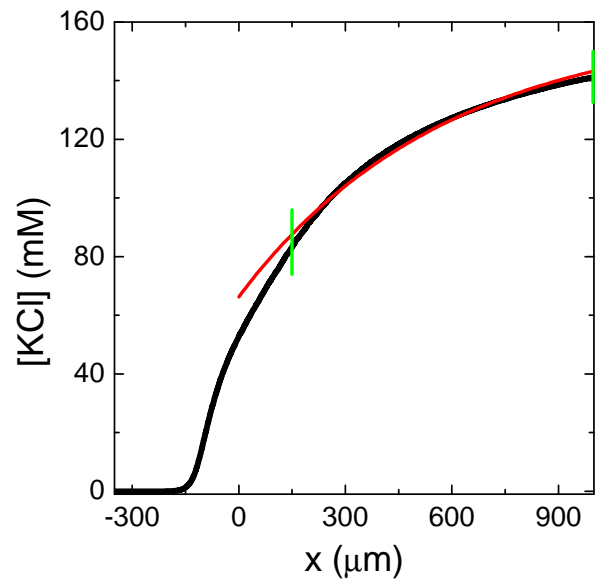

(c)

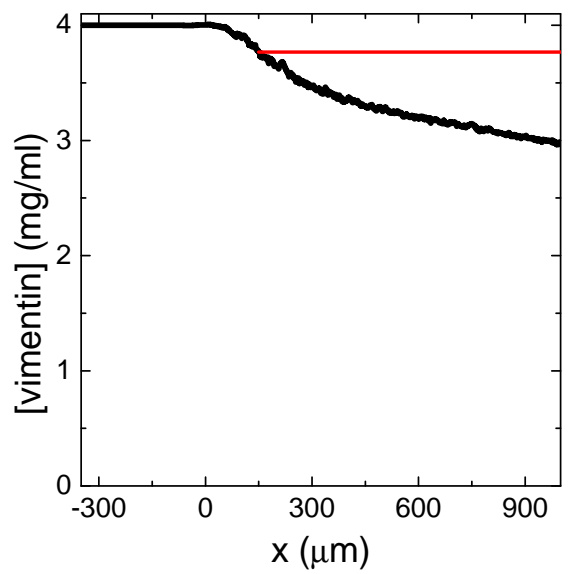

Figure 6.17: Examples of simulation results (black lines) along a single streamline and their extrapolation functions to larger $x$ (red lines) for the streamline starting at $y=75 \mu \mathrm{m}$ and $z=27 \mu \mathrm{m}$. The green lines denote the $x$-range used for determining the extrapolation function. (a) The assembly time is extrapolated by a linear function based on its slope on a fixed $x$-range. (b) The salt concentration is estimated by fitting a double-exponential decay to $160 \mathrm{mM}$ on a fixed $x$-range. (c) The protein concentration is estimated by its value at $x=150 \mu \mathrm{m}$, to compensate for the overestimate of the diffusion constant. See also figure 6.16. 
(a)

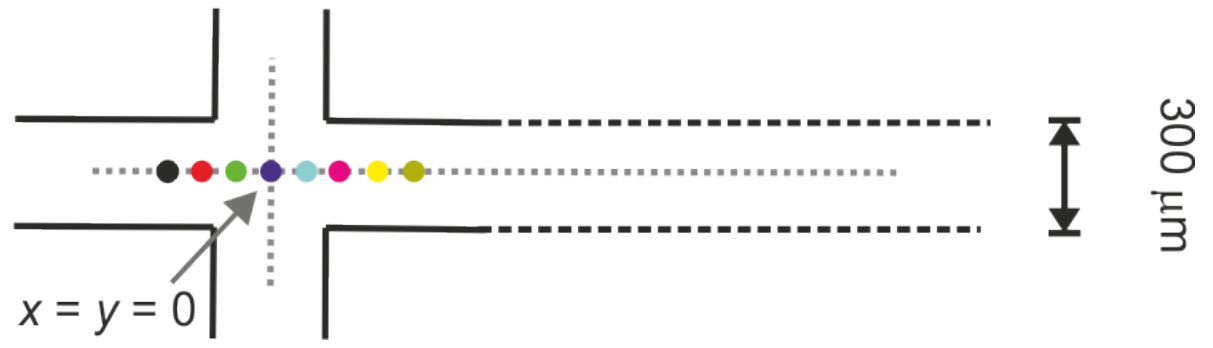

(b)

(c)
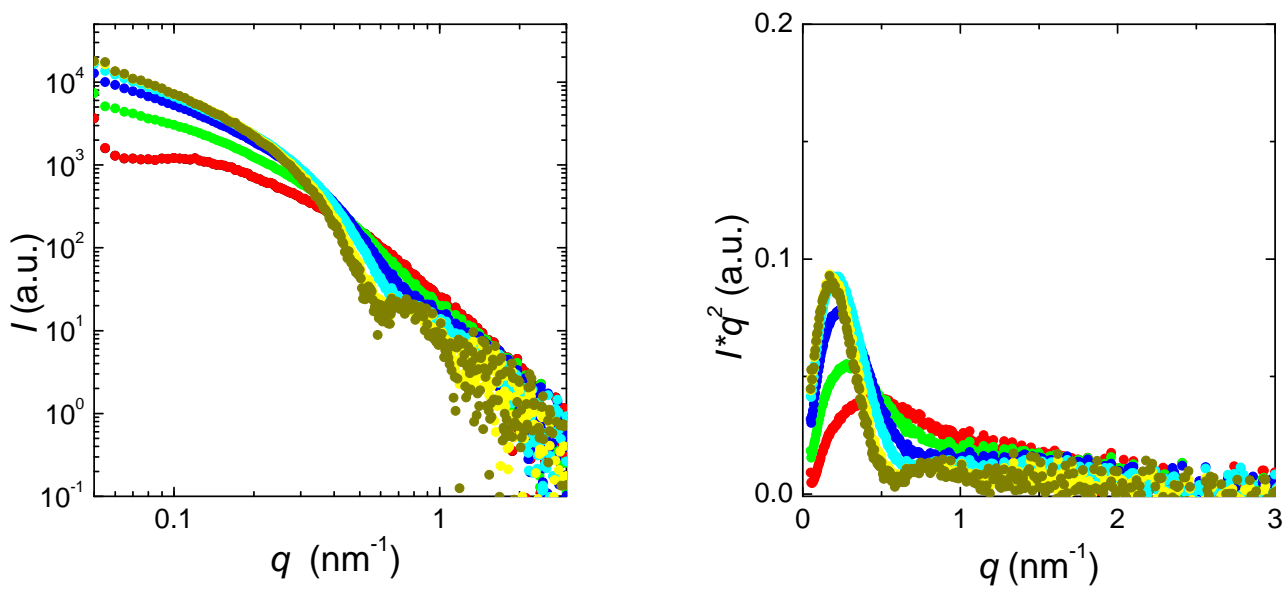

Figure 6.18: Estimate of SAXS curves in microflow based on stationary wild type data for the simple cross geometry with flow speeds of $335 \mu \mathrm{m} / \mathrm{s}(40 \mu \mathrm{l} / \mathrm{h})$ for the vimentin solution and a mean flow speed of the salt buffer solution $([\mathrm{KCl}]=200 \mathrm{mM})$ in the inlet of $310 \mu \mathrm{m} / \mathrm{s}(80 \mu \mathrm{l} / \mathrm{h})$, see figure 6.3. (a) Localization of the exemplary probe locations in the microfluidic device. (b) Estimated SAXS signals corresponding to these locations. (c) Kratky plot of the estimated SAXS signals. 
vimentin, as there is no salt present. At the first measurement point in the intersection (green) despite a rather low mean salt concentration and a significant amount of vimentin without any contact to salt, the scattering already significantly changes and resembles the scattering observed in equilibrium in the $10 \mathrm{mM}$ to $20 \mathrm{mM} \mathrm{KCl}$ range. As we proceed further downstream the changes in the scattering are similar to those observed upon increasing the potassium chloride concentration: Increase of the slope of scattering towards small $q$, increase of overall scattering at small $q$ and decrease of scattering at higher $q$. Also, at $x=0$ we can see a change in slope at $0.7 \mathrm{~nm}^{-1}$ which becomes a local minimum-maximum pair at $x=300 \mu \mathrm{m}$. Even further downstream the changes in the curves become less as both the changes in the salt concentration become less and the stationary curves at $80 \mathrm{mM} \mathrm{KCl}$ and $100 \mathrm{mM} \mathrm{KCl}$ are also quite similar (see figure 4.5a). The Kratky plot is also very similar to the equilibrium plot. Noticeably, from $x=100 \mu \mathrm{m}$ onwards, the height of the peak remains constant, although it continues to shift to smaller $q$.

\subsubsection{Instantaneous ULF formation and elongation}

Another approach to estimating the SAXS signal is to assume that vimentin instantaneously forms ULFs and starts to elongate as it comes in contact with salt, but that compaction to mature filaments proceeds much slower. This is quite reasonable when one compares the typical timescales for these processes based on the TEM experiments $[35,44]$. In this case, the scattering at a fixed salt concentration should correspond to the scattering of the $Y 117 \mathrm{~L}$ mutant in equilibrium and we can calculate estimates of the scattering for different locations in the flow as above by simply replacing the data from the wild type with data from the mutant.

When we compare the estimated curves in figure 6.19, we see that for $x \leq 0$ the resulting curves are very similar to those we calculated based on the wild type data. This is no surprise, as the equilibrium curves at low salt concentrations are also very similar, see figure 4.9. However, as we go further downstream, differences emerge: The scattering at high $q$-values decreases less and instead of local minima and maxima we obtain the S-like curve shape we also observe for the equilibrium state of $Y 117 L$, see figure 4.7. We can understand why the decrease of scattering at high $q$ is reduced when we consider figure 4.9c: For higher salt concentrations, the scattering at high $q$ of the $Y 117 \mathrm{~L}$ mutant in equilibrium is above that of the wild type at identical salt concentration. As at lower salt concentrations the scattering at high $q$ is (nearly) identical for both cases, it follows that the estimate scattering at high $q$ based in the mutant data is always equal or higher than the estimate based on 
(a)

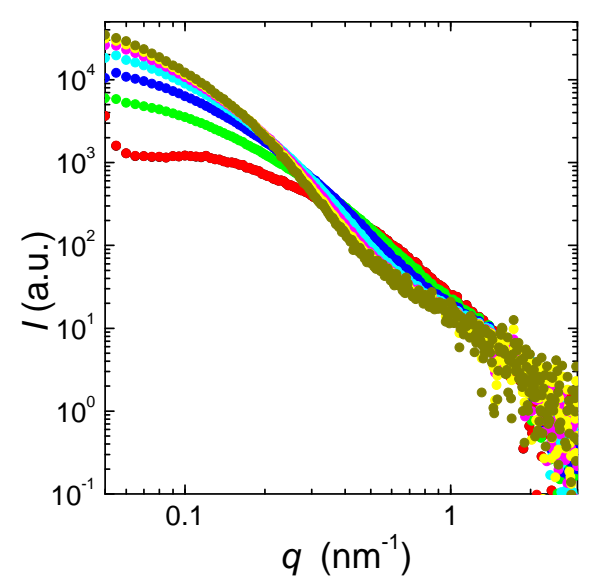

(b)

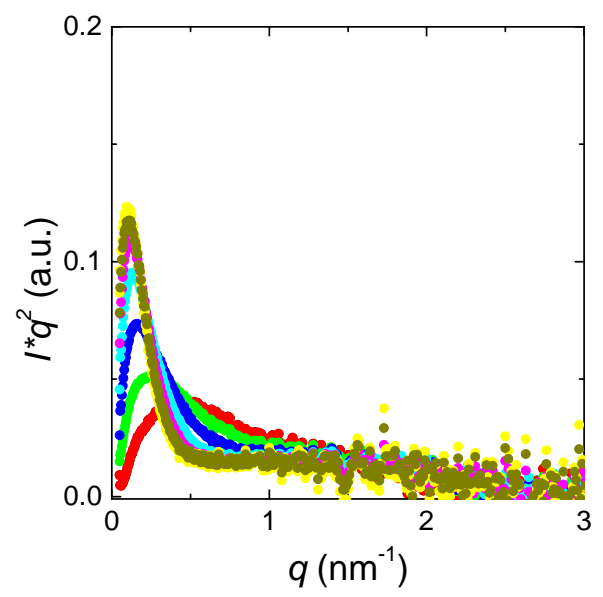

Figure 6.19: Estimate of SAXS curves in microflow based on stationary $Y 11^{7} / \mathrm{L}$ data for the simple cross geometry with flow speeds of $335 \mu \mathrm{m} / \mathrm{s}(40 \mu \mathrm{l} / \mathrm{h})$ for the vimentin solution and a mean flow speed of the salt buffer solution $([\mathrm{KCl}]=200 \mathrm{mM})$ in the inlet of $310 \mu \mathrm{m} / \mathrm{s}(80 \mu \mathrm{l} / \mathrm{h})$, see figure 6.3. The color code is identical to the one in figure 6.18. (a) Estimated SAXS signals corresponding to these locations. (b) Kratky plot of the estimated SAXS signals.

the wild type data. In the Kratky plot, we already can identify differences between the $Y 117 \mathrm{~L}$ mutant data based estimates and the wild type data based estimates at $x=0$. Whereas in the latter case the peak is rather symmetric, the peak in the $Y 11^{r} \mathrm{~L}$ data is quite slanted towards smaller $q$. Further downstream, the peaks become symmetric, but growth of peak height levels off at a lower rate than for the wild type based data. The assymetry in the peak is a feature that is absent in the equilibrium data (figure $4.7 \mathrm{~b}$ ), i.e. it uniquely stems from the superposition of states in the probe volume.

These findings imply that compaction to mature filaments (see section 2.1.2) alters the shapes of the curves in flow at a significant level and in principle could be distinguished in the experiment.

\subsubsection{Instantaneous ULF formation without elongation}

When we go one step further back in the assembly process, we consider the instantaneous formation of ULFs which do not elongate on the observed timescales. As our results of chapter 4 indicate, even for the $Y 117 \mathrm{~L}$ this does not correspond to the equilibrium state and we need to model the corresponding SAXS curves for all salt concentrations. We begin by estimating the scattering signal for those salt concen- 
trations at which data of the $Y 11^{\prime} 7 \mathrm{~L}$ mutant was recorded. We assume that actual ULFs are $L=60 \mathrm{~nm}$ long [30] and have the same cross-section as the equilibrium state of $Y 117 L$. We can then assume that the scattering signals for the ULF $I_{U L F}$ and the mutant $I_{Y 117 L}$ only differ in the longitudinal contribution (see equation 3.7 ) and are therefore related via

$$
I_{U L F}(q, c s)=\left(2 \frac{\operatorname{Si}(q L)}{q L}-\left(\frac{\sin (q L / 2)}{q L / 2}\right)^{2}\right) \cdot \frac{q L}{\pi} \cdot I_{Y 117 L}(q, c s)
$$

see equations 3.8 and 3.10. These curves then allow us to estimate the scattering from the other relevant salt concentrations by employing the same interpolation and extrapolation methods as for the two cases discussed above.

As we already could expect from figure 4.10, we see that the effect of the theoretical

(a)

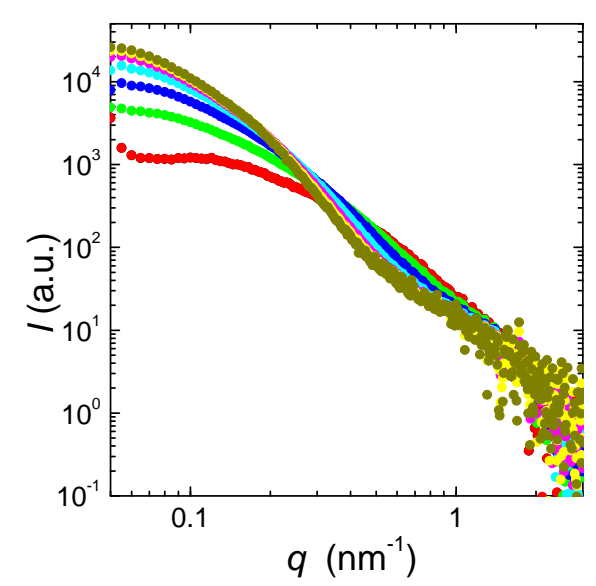

(b)

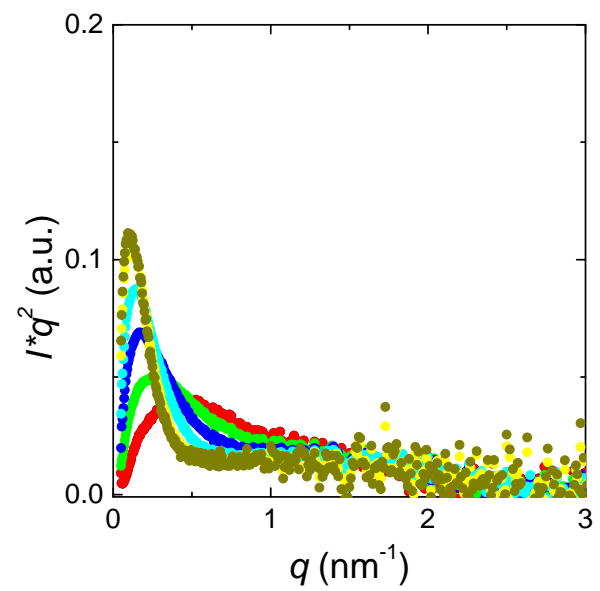

Figure 6.20: Estimate of SAXS curves in microflow based on length adjusted stationary $Y 117 \mathrm{~L}$ data for the simple cross geometry with flow speeds of $335 \mu \mathrm{m} / \mathrm{s}(40 \mu \mathrm{l} / \mathrm{h})$ for the vimentin solution and a mean flow speed of the salt buffer solution $([\mathrm{KCl}]=200 \mathrm{mM})$ in the inlet of $310 \mu \mathrm{m} / \mathrm{s}(80 \mu \mathrm{l} / \mathrm{h})$, see figure 6.3. The color code is identical to the one in figure 6.18. (a) Estimated SAXS signals corresponding to these locations. (b) Kratky plot of the estimated SAXS signals.

change in length is minor (figure 6.20): only scattering towards small $q$ is very slightly reduced, by less than a factor of 1.5. In the Kratky plots no changes can be identified. This implies that in contrast to compaction, elongation can barely be resolved and most likely will remain hidden behind other structural changes. 


\subsubsection{From tetramer to filament including kinetics}

Finally, we can consider the fact that none of the processes discussed above is instantaneous or infinitely slow. This leads us to the need to model the reaction kinetics of vimentin from tetramers to compacted filaments. We base our model on the model proposed by Kirmse, Portet et al. [35, 47,48], but distinguish only species which at least theoretically might be distinguishable in SAXS: Tetramers $T$, octamers $O$, hexadecamers $H$, ULFs $U_{1}$, two and three ULF aggregates $U_{2}, U_{3}$, uncompacted filaments $U_{U}$ and compacted filaments $F$. The basic reactions are lateral assembly of two tetramers, octamers or hexadecamers to octamers, hexadecamers and ULFs, respectively, the longitudinal annealing of ULFs, ULF aggregates and uncompacted filaments to each other and the compaction of filaments to mature filaments. The rate equations for the model then become

$$
\begin{aligned}
& \frac{d T}{d t}=-2 k_{l} T^{2} \\
& \frac{d O}{d t}=k_{l} T^{2}-2 k_{l} O^{2} \\
& \frac{d H}{d t}=k_{l} O^{2}-2 k_{l} H^{2} \\
& \frac{d U_{1}}{d t}=k_{l} H^{2}-2 k_{a} U_{1}^{2}-k_{a} U_{1} U_{2}-k_{a} U_{1} U_{3}-k_{a} U_{1} U_{U} \\
& \frac{d U_{2}}{d t}=k_{a} U_{1}^{2}-2 k_{e} U_{2}^{2}-k_{a} U_{1} U_{2}-k_{e} U_{2} U_{3}-k_{e} U_{2} U_{U} \\
& \frac{d U_{3}}{d t}=k_{a} U_{1} U_{2}-2 k_{e} U_{3}^{2}-k_{a} U_{1} U_{3}-k_{e} U_{2} U_{3}-k_{e} U_{3} U_{U} \\
& \frac{d U_{U}}{d t}=k_{a} U_{1} U_{3}+k_{e} U_{2}^{2}+k_{e} U_{3}^{2}-k_{c} U_{U} \\
& \frac{d F}{d t}=k_{c} U_{U}
\end{aligned}
$$

with the rate constant for lateral assembly $k_{l}=20 \mu \mathrm{M}^{-1} \mathrm{~s}^{-1}$, elongation by addition of one ULF $k_{a}=1.7 \mu \mathrm{M}^{-1} \mathrm{~s}^{-1}$, elongation by fusion of extended objects $k_{e}=0.11 \mu \mathrm{M}^{-1} \mathrm{~s}^{-1}$ and compaction $k_{c}=5 \mathrm{~s}^{-1} . k_{l}, k_{a}$ and $k_{e}$ are based on the results from Kirmse, Portet et al. [35,48], whereas the compaction rate was estimated from the final filament diameter and the filament diameter at $10 \mathrm{~s}$ given in [44] assuming an initial ULF diameter of $17 \mathrm{~nm}$. This model ignores a number of possi- 
ble processes, such as octamer - hexadecamer aggregation or elongation of mature filaments, but nevertheless should provide us with some idea on how intermediate steps might change the SAXS signal.

Figure 6.21a shows the concentrations of the considered species in dependence of

(a)

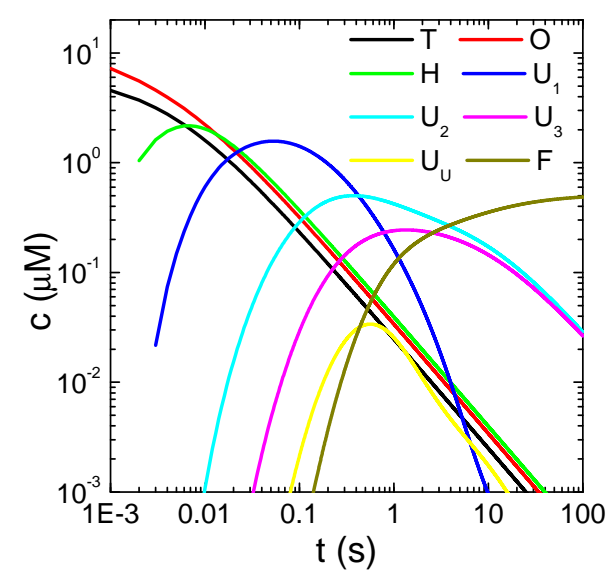

(b)

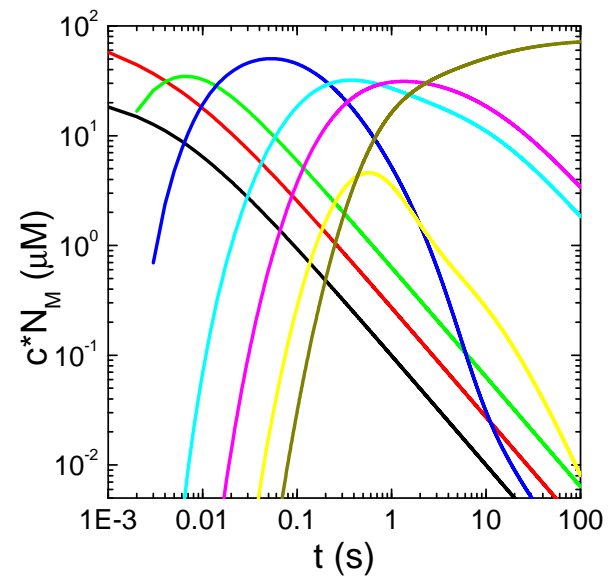

Figure 6.21: Solution of the rate equations 6.5 to 6.12 for vimentin assembly. (a) Concentration of each considered species. (b) Number of vimentin tetramers in each state. At $10 \mathrm{~s}$ compacted filaments are the dominant species by both molarity and mass.

the assembly time. The initial tetramer concentration is $20 \mu \mathrm{M}$ corresponding to $4 \mathrm{mg} / \mathrm{ml}$ of protein, and the size of a time step is $1 \mathrm{~ms}$. The hexadecamer concentration peaks at about $6 \mathrm{~ms}$, ULFs at about $50 \mathrm{~ms}$, non-compacted filaments at about half a second. The compact filament concentration exceeds $1 \mathrm{nM}$ for the first time at $125 \mathrm{~ms}$. It is important to note that what is shown here is the molar concentration. As the mass of a filament is considerably larger than that of a tetramer, the amount of vimentin in each higher aggregation state is considerably larger and so is the contribution to the overall scattering of each state. For the filamentous states (pre-compaction and compacted) we additionally need to estimate the total amount of vimentin monomers incorporated in them. Figure $6.21 \mathrm{~b}$ shows how the amount of vimentin monomers found in each of the individual states changes over time. We see that at about $50 \mathrm{~ms}$ there is nearly an order of magnitude more vimentin in the ULF state than in any other state and that the only other state which dominates at any time are compacted filaments. Also, starting from $50 \mathrm{~ms}$ onwards, the amount of vimentin in smaller oligomers is so small that its contribution to the scattering signal is most likely minor. As already at $x=0$ the smallest assembly time in the probe volume exceeds $500 \mathrm{~ms}$ (see figure 6.4b) we can conclude that our previous 
assumption of instantaneous ULF formation was not too far from reality.

In order to model SAXS curves from these results we need to find a model for

(a)

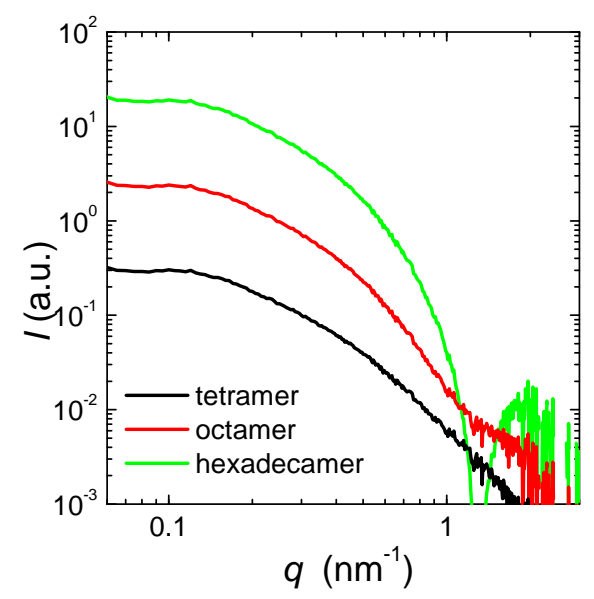

(b)

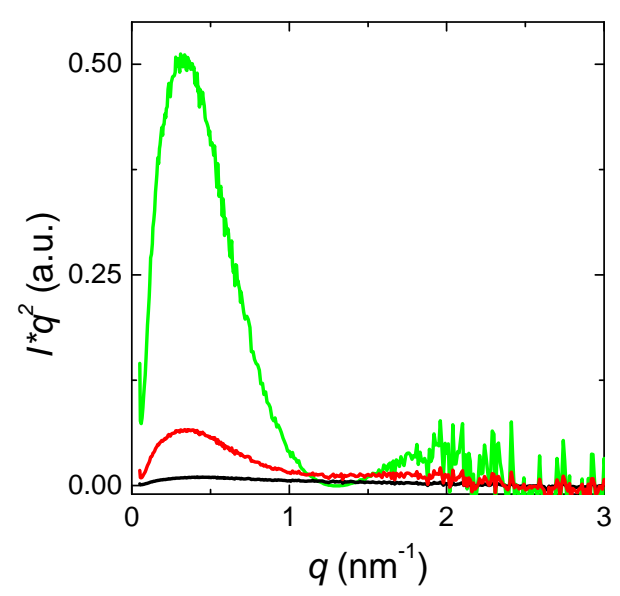

Figure 6.22: (a) Modeled scattering curves of individual octameric (red) and hexadecameric (green) vimentin and measured scattering of tetrameric (black) vimentin in comparison. The modeled curves are based on equations B.7 to B.10. (b) Kratky plot of the estimated SAXS signals.

each individual species. For the ULFs and elongated states we can use the models discussed above, but for the octamers and hexadecamers we can only estimate the SAXS signal based on geometric assumptions and the tetrameric scattering signal. We model octamers as two parallel tetramers at $d=3.2 \mathrm{~nm}$ distance and hexadecamers as a square of four parallel tetramers with a side length of $d$ [30]. The derivation of the corresponding SAXS signals is given in appendix B.4. In figure 6.22 we see that the larger the aggregate the larger the forward scattering and the steeper the decay towards larger $q$. Slightly above $1 \mathrm{~nm}^{-1}$ the octamers show a slight minimum and the hexadecameric signal nearly vanishes due to destructive interference of scattering from the contributing tetramers. In the Kratky plot (figure 6.22b) the peak of octamers lies at $0.35 \mathrm{~nm}^{-1}$ and the peak of the hexadecamers at $0.32 \mathrm{~nm}^{-1}$, i.e. roughly at the same position as the peak of the tetramers. In contrast to the larger aggregates we do not assume that the scattering from the oligomers and hexadecamers depends on the salt concentration.

Figure $6.23 \mathrm{~b}$ shows the SAXS curves that result from the above considerations. Interestingly, including reaction kinetics in the estimate results in a significantly larger change in the scattering signal between $x=-200 \mu \mathrm{m}$ and $x=-100 \mu \mathrm{m}$, i.e. upon entering the intersection, than in all previously discussed cases. Further down- 
(a)

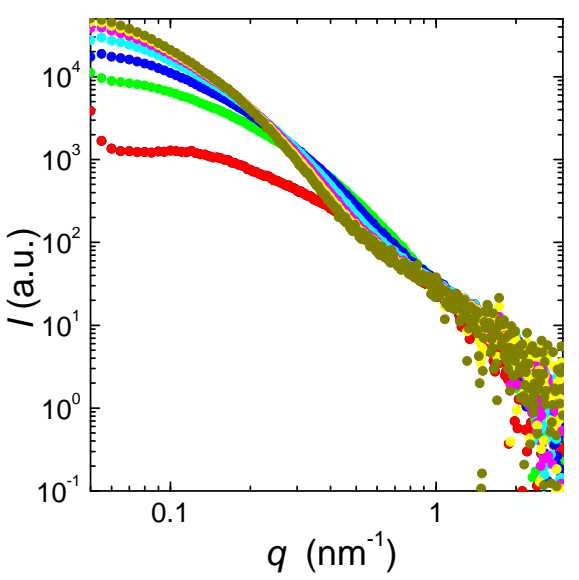

(b)

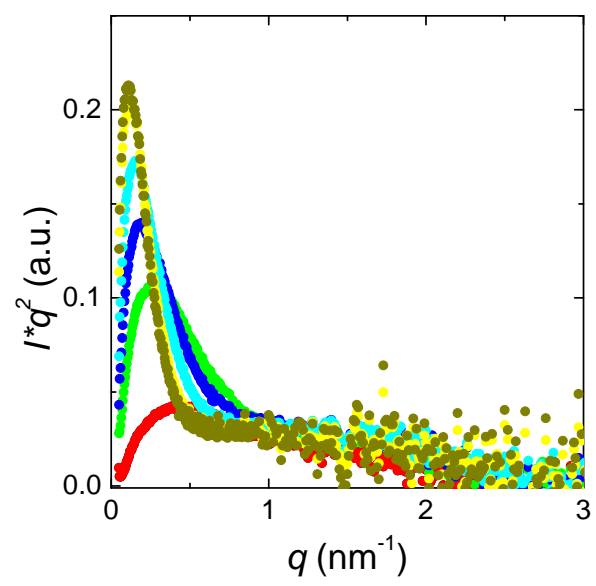

Figure 6.23: Estimate of SAXS curves in microflow based on equations 6.5 to 6.12 for the simple cross geometry with flow speeds of $335 \mu \mathrm{m} / \mathrm{s}(40 \mu \mathrm{l} / \mathrm{h})$ for the vimentin solution and a mean flow speed of the salt buffer solution $([\mathrm{KCl}]=200 \mathrm{mM})$ in the inlet of $310 \mu \mathrm{m} / \mathrm{s}$ $(80 \mu \mathrm{l} / \mathrm{h})$, see figure 6.3. The color code is identical to figure 6.18. (a) Estimated SAXS signals corresponding to these locations. (b) Kratky plot of the estimated SAXS signals.

stream, we can observe an increase of the slope towards smaller $q$ which corresponds to the elongation from $60 \mathrm{~nm}$ long intermediates to longer states. Additionally, the forward scattering increases further than in the previously discussed cases, and the scattering at high $q$ values remains nearly constant. As the mean assembly time at $x=400 \mu \mathrm{m}$ (dark yellow circles) is $13 \mathrm{~s}$ we would expect the scattering curve to resemble the curve observed for mature filaments, as there is about three times as much protein in mature filaments than in un-compacted filaments at $13 \mathrm{~s}$ (see figure 6.21b). However, the final curve closely resembles that of the non-compacted filaments (see figure 6.19a), with a slightly less pronounced shape. This might be due to the fact that over a broad $q$ range the $Y 117 \mathrm{~L}$ mutant actually scatters more strongly than the wild type (see figure 4.9c) and hence scattering from un-compacted filaments can smear out the local structures from the scattering of mature filaments. In the Kratky plot, we see an initial increase of the peak height and then a shift towards smaller $q$, accompanied by continued peak growth. At $x=-100 \mu \mathrm{m}$ the peak is slightly asymetric but considerably higher than in the previously discussed cases. Further downstream, the peak is always higher and narrower than in the previous cases.

This implies that under these flow conditions it might be possible to identify elongation as well as earlier lateral changes in the structure. 


\section{Chapter 7}

\section{Assembly of vimentin in microflow}

In this chapter, we show the results from the vimentin assembly experiments in microflow as introduced in sections 5.7 and 3.3.2 at different flow conditions. We compare the scattering curves to the equilibrium results (chapter 4) and the predicted curves for different assembly models (chapter 6). Finally we quantify the observed changes in the scattering pattern and how changes in the flow conditions affect the assembly process.

\subsection{Exemplary results}

We begin the discussion of the actual experimental data by considering the flow conditions which we discussed the most thoroughly in the previous chapter: A simple cross geometry of $300 \mu \mathrm{m}$ wide channels with a mean flow speed of $335 \mu \mathrm{m} / \mathrm{s}$ $(40 \mu \mathrm{l} / \mathrm{h})$ for the vimentin solution and a mean flow speed of the salt buffer solution in the inlet of $310 \mu \mathrm{m} / \mathrm{s}(80 \mu \mathrm{l} / \mathrm{h})$. The measured protein concentration is $4.1 \mathrm{mg} / \mathrm{ml}$ and the buffer salt concentration is $200 \mathrm{mM} \mathrm{KCl}$. The buffer system is a $4 \mathrm{mM}$ phosphate buffer at $\mathrm{pH} 7.5$.

When we consider the changes in the scattering close to the intersection region of the cross in figure 7.1b, as soon as the vimentin enters the intersection and has contact to salt, the scattering at small $q$ increases and the increase of scattering towards $q=0$ becomes steeper. This effect is similar to the stationary results on increasing potassium chloride concentration (figure 4.5a). Starting from $x=-100 \mu \mathrm{m}$

the curves share an isoscattering point at $0.15 \mathrm{~nm}^{-1}$. At higher $q$, the scattering also initially increases and than returns to lower values as the curves steepen. This upward shift of the scattering upon entering the intersection might be due to a higher 
(a)

$$
\Delta x=100 \mu \mathrm{m} \quad \Delta x=5 \mathrm{~mm}
$$

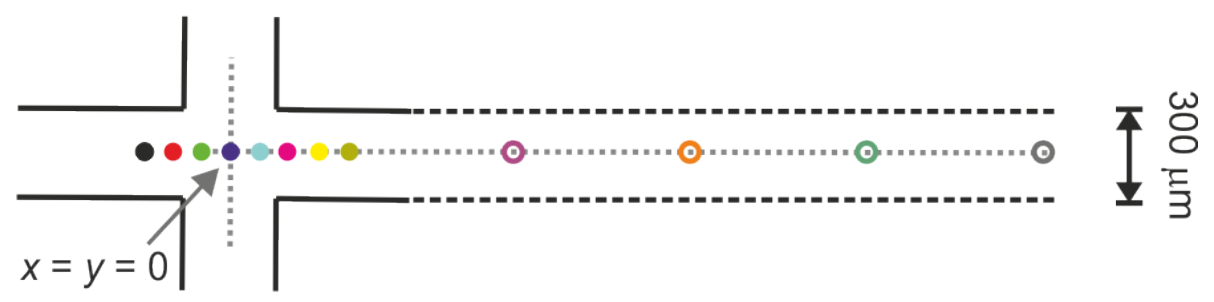

(b)

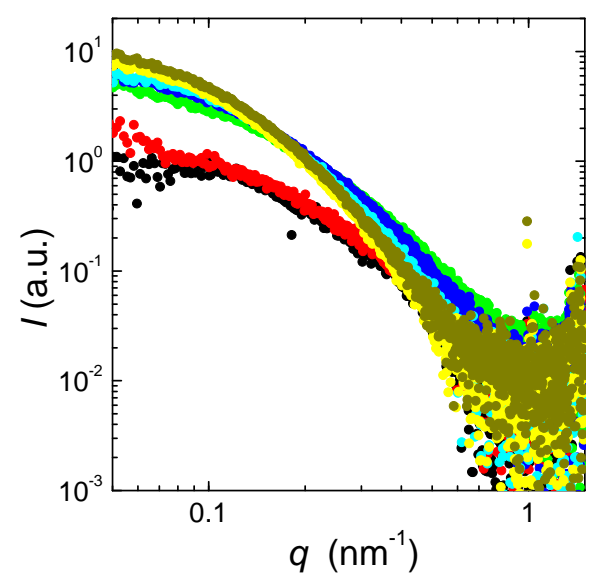

(c)

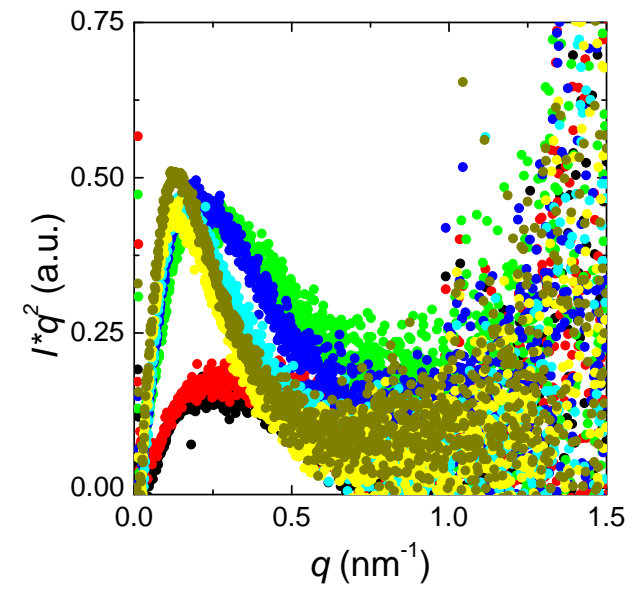

Figure 7.1: SAXS curves in microflow for the simple cross geometry with flow speeds of $335 \mu \mathrm{m} / \mathrm{s}(40 \mu \mathrm{l} / \mathrm{h})$ for the vimentin solution and a mean flow speed of the salt buffer solution $([\mathrm{KCl}]=200 \mathrm{mM})$ in the inlet of $310 \mu \mathrm{m} / \mathrm{s}(80 \mu \mathrm{l} / \mathrm{h})$ (a) Localization of the exemplary probe locations in the microfluidic device. The closed and open symbols are not on the same scale. (b) SAXS signals recorded positions close to the intersection. (c) Corresponding Kratky plots. 
amount of protein passing through the probe volume during exposure because of the hydrodynamic focusing. Noticeably, we see no emergence of structure in the curves as we saw for the equilibrium data and the simulated curves. When we consider the Krakty plots we see an instantaneous increase in the height of the peak and narrowing of its width with the peak location remaining at about $0.3 \mathrm{~nm}^{-1}$. Further downstream, the peak height remains on the same level while its position shifts to smaller $q$ and it further narrows and becomes more symmetric. At $x=500 \mu \mathrm{m}$ the peak is located at about $0.15 \mathrm{~nm}^{-1}$.

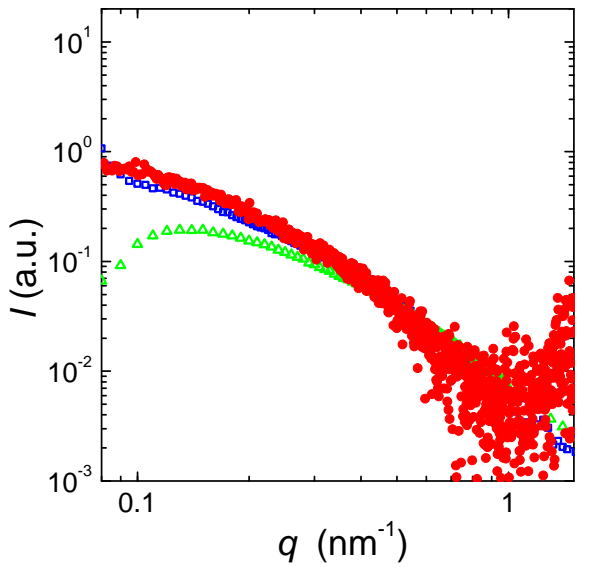

Figure 7.2: Comparison of equilibrium and in-flow low salt data of vimentin: The red circles are the SAXS signal recorded before the intersection in flow, the blue squares result from identical buffer conditions ( $4 \mathrm{mM}$ phosphate) in equilibrium and the green triangles correspond to the buffer conditions used for the equilibrium studies in chapter 4 ( $2 \mathrm{mM}$ phosphate). The inflow curve is scaled to comparable units. The differences are most likely due to an increased amount of ions in the $4 \mathrm{mM}$ phosphate buffer.

and thereby reduce systematic inter-tetramer scattering effects that lower the scattering at low $q$, leading to an overall effective increase of scattering. The latter could possibly induce some slight pre-assembly of vimentin resulting in a stronger scattering. However, as the scattering at higher $q$ is considerably less changed, we assume that the largest contribution to the signal change stems from the first effect
Before we consider how the scattering changes as we continue even further downstream, let us compare the low salt data to our equilibrium results (figure 7.2). When we compare the data recorded in the protein inlet to the data we discussed in chapter 4 , we find a considerable difference in the scattering at low $q$. However, the data discussed in chapter 4 were recorded at different buffer conditions than the data recorded in the inlet $(2 \mathrm{mM}$ phosphate as opposed to $4 \mathrm{mM}$ phosphate). The comparison of the data from the inlet to equilibrium data recorded at the same buffer conditions shows that the curves agree rather well. Therefore, we conclude that the changes are due to the change in phosphate concentration and could be induced by either the general increase in ion concentration or in particular by the increase of sodium ions in the $4 \mathrm{mM}$ phosphate system. The first phenomenon would increase the electrostatic screening - 
and that the vimentin remains tetrameric even at $4 \mathrm{mM}$ phosphate.

When we compare the scattering curves even further downstream (figure 7.3), we

(a)

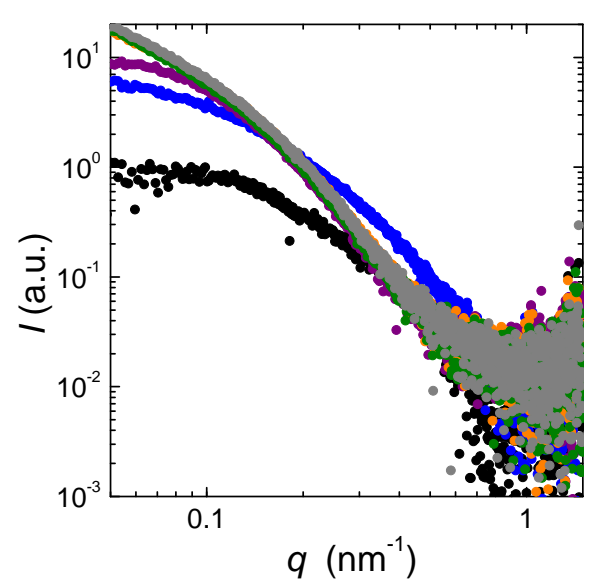

(b)

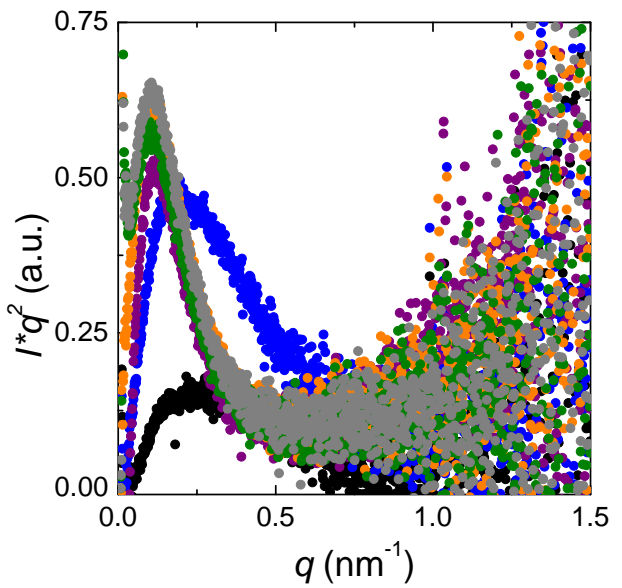

Figure 7.3: SAXS curves in microflow for the simple cross geometry with flow speeds of $335 \mu \mathrm{m} / \mathrm{s}(40 \mu \mathrm{l} / \mathrm{h})$ for the vimentin solution and a mean flow speed of the salt buffer solution $([\mathrm{KCl}]=200 \mathrm{mM})$ in the inlet of $310 \mu \mathrm{m} / \mathrm{s}(80 \mu \mathrm{l} / \mathrm{h})$ (a) SAXS signals recorded positions close to the intersection and further downstream. (b) Corresponding Kratky plots.

see that from $x=0$ to $x=5 \mathrm{~mm}$ the changes in the scattering below $q=0.35 \mathrm{~nm}^{-1}$ follow the same trends observed on the first $0.5 \mathrm{~mm}$. At higher $q$, however, a change of slope begins to emerge which becomes more pronounced as we go further downstream. Between $x=1 \mathrm{~cm}$ and $x=2 \mathrm{~cm}$ the only feature in the curves that seems to systematically change is this change in slope and at $x=2 \mathrm{~cm}$ (corresponding to about $225 \mathrm{~s}$ mean assembly time) the scattering resembles the scattering found for the $Y 117 L$ mutant at high salt concentrations. (see figure 4.7).

Indeed, direct comparison of the scattering curves recorded at $2 \mathrm{~cm}$ from the intersection with data for the $Y 11^{\prime} / \mathrm{L}$ mutant recorded at $100 \mathrm{mM} \mathrm{KCl}$ in figure 7.4 shows very good agreement within the noise level despite the use of different mutants, different assembly times (minutes opposed to days), different salt concentrations (160 mM opposed to $100 \mathrm{mM}$ ), and different buffer systems (4 mM phosphate opposed to $2 \mathrm{mM}$ phosphate). This is a very strong indicator that over a length of $2 \mathrm{~cm}$ we can observe lateral assembly to ULFs and possibly subsequent elongation but no compaction. However, in comparison to the estimates as presented in chapter 6 , the changes in the scattering curves appear slower than expected. 


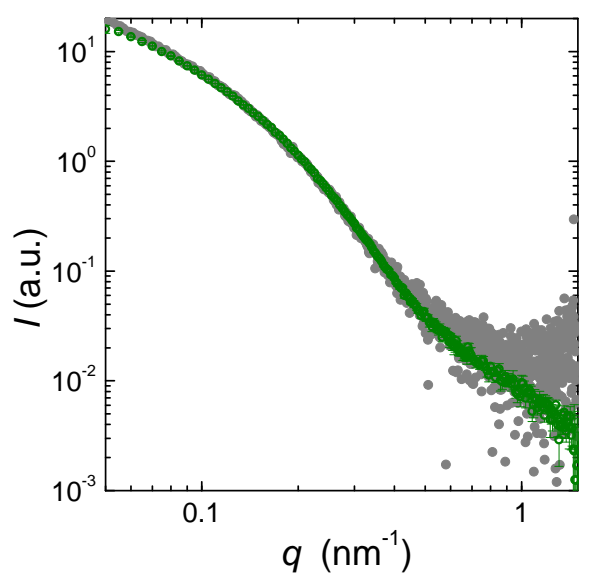

Figure 7.4: Comparison of equilibrium high salt and in-flow long assembly time data of vimentin: The gray circles show the SAXS signal of vimentin wild type recorded $2 \mathrm{~cm}$ downstream from the intersection, the green circles present the equilibrium data of the $Y 177 L$ mutant at $100 \mathrm{mM} \mathrm{KCl}$. The in-flow curve is scaled to comparable units. The small differences are most likely due to an increased scattering of the in-flow data.
Let us now consider what happens when we change the salt concentration in the buffer to $100 \mathrm{mM}$ but maintain the flow speed. The data for this scenario are recorded in a channel with long serpentines (and an effective length of about $15 \mathrm{~cm}$ ) with slightly thicker channel walls at a protein concentration of $3.7 \mathrm{mg} / \mathrm{ml}$ which reduces the signal and hence decreases the effective accessible $q$-range and increases the signal to noise level.

Near the intersection (figure 7.5), we see that due to the causes discussed above, the overall scattering signal is considerably weaker than in the previously discussed case. Next, we see that below $q=1 \mathrm{~nm}^{-1}$ all curves near the intersection flatten off in comparison to those in figure 7.1b. This can either be an artefact of the reduced accessible $q$-range or indeed an effect of the reduced salt and protein concentration. What is similar for both cases is the immediate up-shift of the scattering curve as soon as the protein enters the intersection. Also, we again observe that as the protein flows further downstream its scattering at higher $q$ decreases and that the curves become steeper. However, we cannot clearly distinguish the rise of scattering at lower $q$ and therefore also cannot verify the existence of an isoscattering point. In the Kratky plot, we again see an initial increase in the peak height followed by a shift towards smaller $q$ values. But instead of continuing to increase the peak height actually decreases further downstream. Comparing data taken further downstream, we see some change in the form of the curve between $5 \mathrm{~mm}$ and $1 \mathrm{~cm}$ distance from the intersection but afterwards only the total scaling of the curves changes (figure 7.6a). Analogously, in the Kratky plot the form and position of the peak remain unchanged and only its height changes. In direct comparison to the data recorded in the middle of the intersection (blue circles) we can also see that the slope of the scattering towards $q=0$ becomes steeper, indicating elongation. A possible reason for these strong fluctuations in the scaling of the scattering signal is a slight mis-alignment between the microfluidic device and the X-ray probe: In the serpentine channel the amount 
(a)

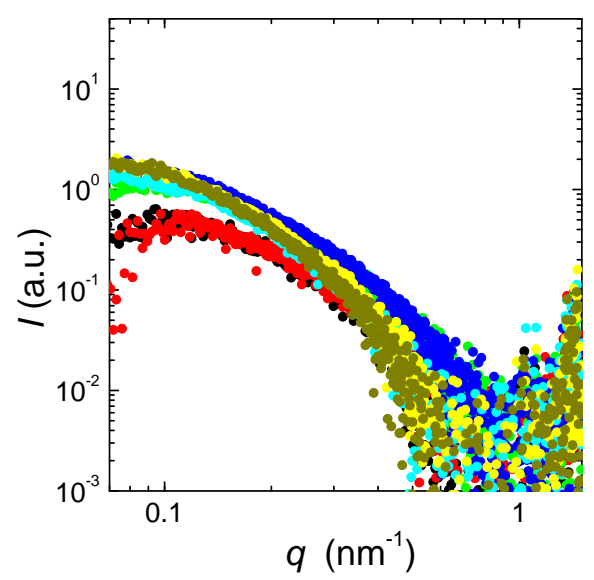

(b)

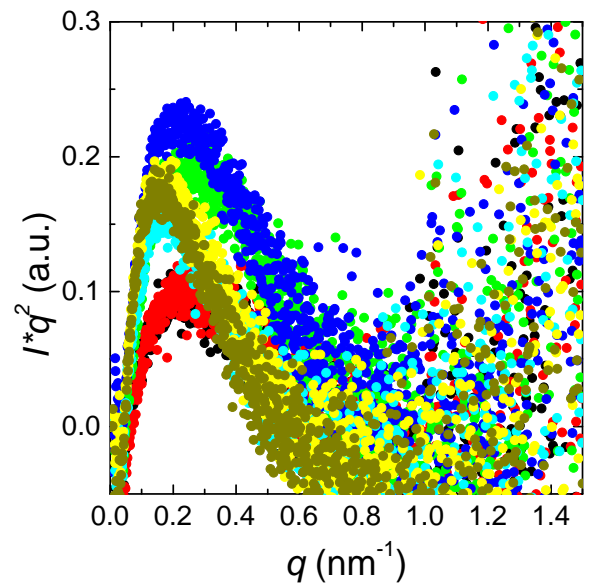

Figure 7.5: SAXS curves in microflow for the simple cross geometry with flow speeds of $335 \mu \mathrm{m} / \mathrm{s}(40 \mu \mathrm{l} / \mathrm{h})$ for the vimentin solution and a mean flow speed of the salt buffer solution $([\mathrm{KCl}]=100 \mathrm{mM})$ in the inlet of $310 \mu \mathrm{m} / \mathrm{s}(80 \mu \mathrm{l} / \mathrm{h})$ (a) SAXS signals recorded positions close to the intersection. (b) Corresponding Kratky plots. The colour code corresponds to figure 7.1a.

of vimentin probed does not only depend on correct placing of the spot in $y$-direction but also in $x$-direction which results in a higher likelihood of probing the flow of the central axis. Also, at longer distances the orientation of the X-ray spot in relation to the main flow-axis is turned by $90^{\circ}$ which also affects the signal. Another factor to consider is that on long time scales (and $17 \mathrm{~cm}$ under the given flow conditions correspond to a mean assembly time of about $3 \mathrm{~min}$ ) diffusion of protein from the jet is most likely no longer negligible, even for long, extended filaments, and also results in an decrease of the scattering signal.

Next, we consider how the the scattering signal changes when we reduce the flow speeds by a factor of four, thereby increasing the effective assembly times at the probe locations by about the same factor, see figure 6.8. As in the previous cases, we observe an instantaneous upwards shift of the overall scattering between preintersection data (black and red circles) and data recorded in the intersection (green circles), see figure 7.6. As we proceed further downstream, the overall scattering curves once again shift downwards. Additionally, there are structural changes in the curves: Already between $x=-100 \mu \mathrm{m}$ and $\mathrm{x}=0$ we see an increase in the slope towards $q=0$ which we only observed much later at higher flow speeds. In the Kratky plot we again see the shift of the peak to smaller $q$ values and as the overall scattering is again accompanied by a decrease in scattering instead of an increase. 
(a)

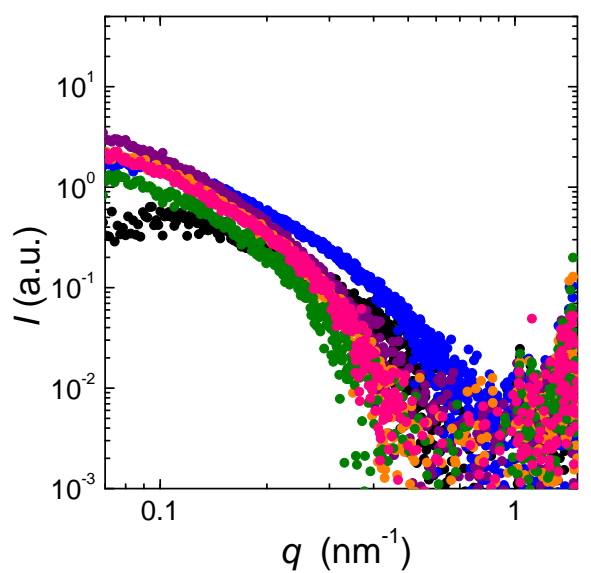

(b)

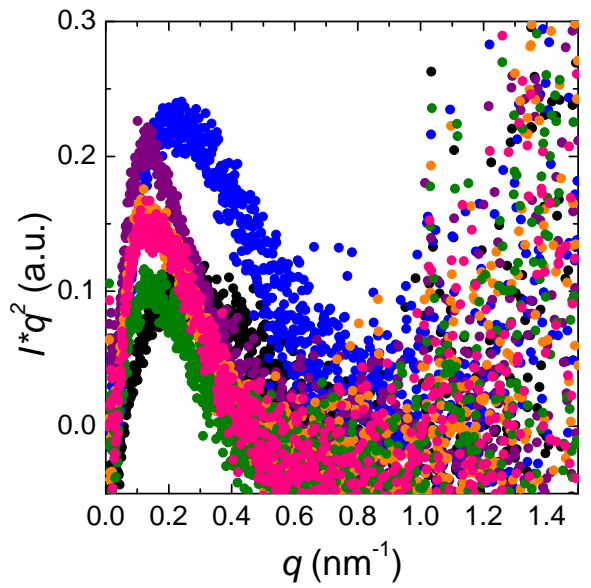

Figure 7.6: SAXS curves in microflow for the simple cross geometry with flow speeds of $335 \mu \mathrm{m} / \mathrm{s}(40 \mu \mathrm{l} / \mathrm{h})$ for the vimentin solution and a mean flow speed of the salt buffer solution $([\mathrm{KCl}]=100 \mathrm{mM})$ in the inlet of $310 \mu \mathrm{m} / \mathrm{s}(80 \mu \mathrm{l} / \mathrm{h})(\mathrm{a})$ Exemplary SAXS signals recorded as the protein flows downstream. (b) Corresponding Kratky plots. The colour code corresponds to figure $7.1 \mathrm{a}$, the pink curve is recorded at $x \approx 17 \mathrm{~cm}$.

(a)

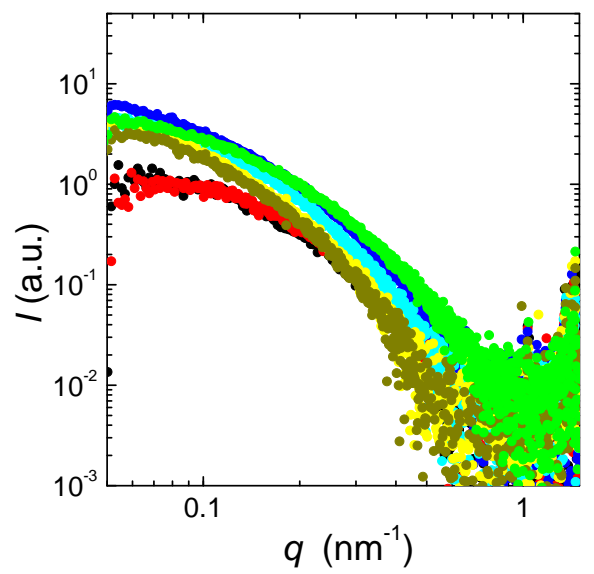

(b)

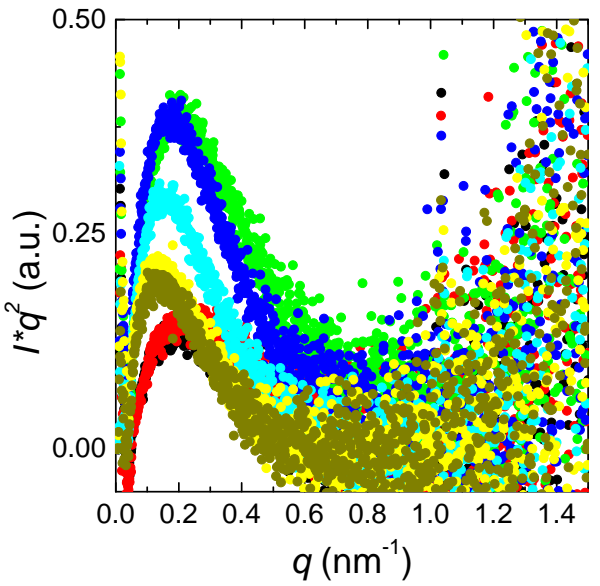

Figure 7.7: SAXS curves in microflow for the simple cross geometry with flow speeds of $84 \mu \mathrm{m} / \mathrm{s}(10 \mu \mathrm{l} / \mathrm{h})$ for the vimentin solution and a mean flow speed of the salt buffer solution $([\mathrm{KCl}]=100 \mathrm{mM})$ in the inlet of $78 \mu \mathrm{m} / \mathrm{s}(20 \mu \mathrm{l} / \mathrm{h})$ (a) SAXS signals recorded at positions close to the intersection. (b) Corresponding Kratky plots. The colour code corresponds to figure 7.1a. 
As we continue further downstream, we can observe a change in the shape of the scattering curve between $5 \mathrm{~mm}$ and $1 \mathrm{~cm}$ from the intersection but afterwards the curves only shift downwards (figure 7.7).

Finally, we consider results obtained upon changing the relative flow speeds, i.e. at

(a)

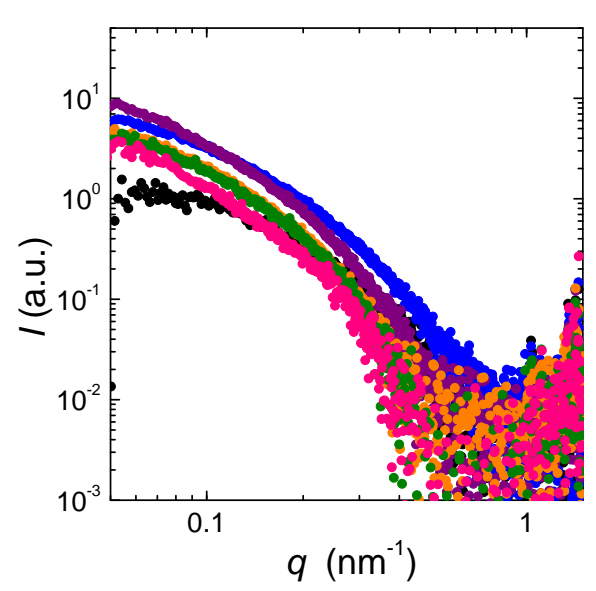

(b)

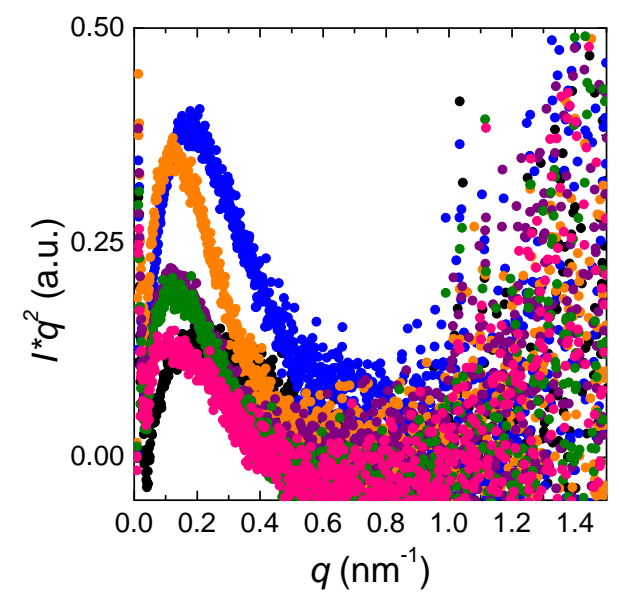

Figure 7.8: SAXS curves in microflow for the simple cross geometry with flow speeds of $84 \mu \mathrm{m} / \mathrm{s}(10 \mu \mathrm{l} / \mathrm{h})$ for the vimentin solution and a mean flow speed of the salt buffer solution $([\mathrm{KCl}]=100 \mathrm{mM})$ in the inlet of $78 \mu \mathrm{m} / \mathrm{s}(20 \mu \mathrm{l} / \mathrm{h})$ (b) SAXS signals recorded positions further downstream. (b) Corresponding Kratky plots. The colour code corresponds to figure $7.1 \mathrm{a}$, the pink curve was recorded at $x \approx 17 \mathrm{~cm}$.

a protein flow speed of $335 \mu \mathrm{m} / \mathrm{s}(40 \mu \mathrm{l} / \mathrm{h})$ and a salt buffer flow speed of $155 \mu \mathrm{m} / \mathrm{s}$ $(40 \mu \mathrm{l} / \mathrm{h})$. For these results the protein concentration remains unchanged but the use of another device with a thinner NOA 81 layer above and below the channel results in an improved signal quality at low $q$ (figure 7.9).

Once again, we see an instantaneous increase in the scattering upon entering the intersection. After that, the curves remain very similar but the scattering at small $q$ seems to continuously increase and the scattering at higher $q$ continuously decreases, just as we observed for the previously discussed flow conditions. In the Kratky plots, we can again identify an increase in the peak height followed by a shift of its location to smaller $q$ which appears weaker than in the previous cases. Also, the narrowing of the peak we previously observed does not seem to emerge on this length scale. On longer distances (figure 7.10) we again observe a shifting in the overall height in the curves which as discussed above is most likely due to technical reasons. Beyond that the slope of the scattering towards $q=0$ increases, indicating elongation. Additionally, the slope of the curve in the mid- $q$ range in- 
(a)

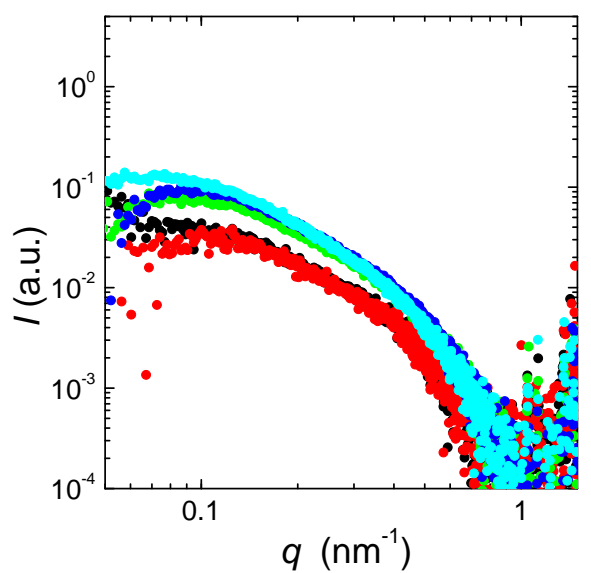

(b)

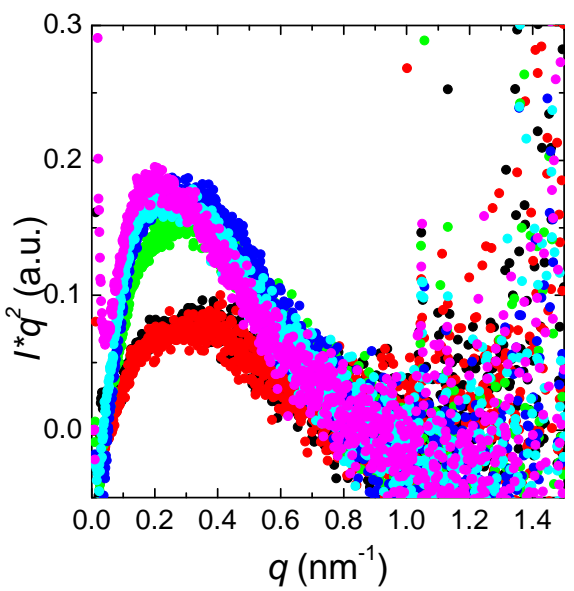

Figure 7.9: SAXS curves in microflow for the simple cross geometry with flow speeds of $335 \mu \mathrm{m} / \mathrm{s}(40 \mu \mathrm{l} / \mathrm{h})$ for the vimentin solution and a mean flow speed of the salt buffer solution $([\mathrm{KCl}]=100 \mathrm{mM})$ in the inlet of $155 \mu \mathrm{m} / \mathrm{s}(40 \mu \mathrm{l} / \mathrm{h})$ (a) SAXS signals recorded positions near the intersection. (b) Corresponding Kratky plots. The colour code corresponds to figure 7.1a.

(a)

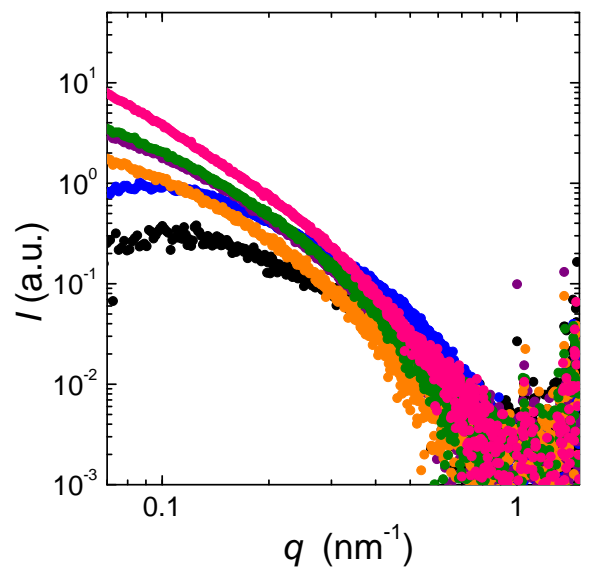

(b)

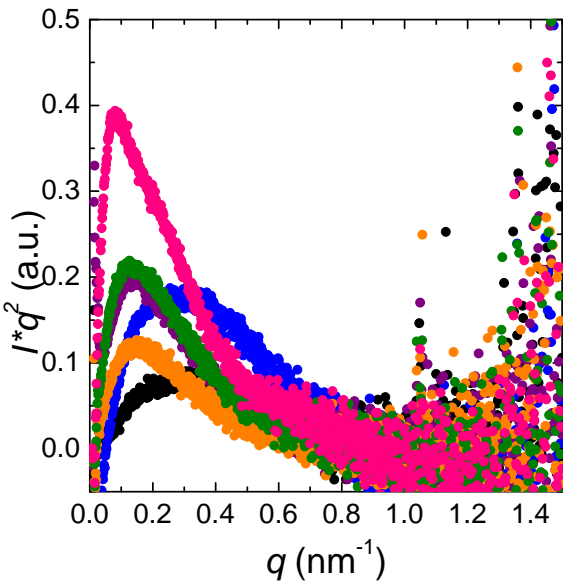

Figure 7.10: SAXS curves in microflow for the simple cross geometry with flow speeds of $335 \mu \mathrm{m} / \mathrm{s}(40 \mu \mathrm{l} / \mathrm{h})$ for the vimentin solution and a mean flow speed of the salt buffer solution $([\mathrm{KCl}]=100 \mathrm{mM})$ in the inlet of $155 \mu \mathrm{m} / \mathrm{s}(40 \mu \mathrm{l} / \mathrm{h})($ a) SAXS signals recorded positions further downstream. (b) Corresponding Kratky plots. The colour code corresponds to figure $7.1 \mathrm{a}$, the pink curve was taken at $x \approx 17 \mathrm{~cm}$. 
creases, which, as we saw in chapter 4, is a sign for lateral growth. In the Kratky plot, the peak shifts to smaller $q$ when going from $x=0$ to $x=5 \mathrm{~mm}$, then remains on the same location up to $x=15 \mathrm{~mm}$ but at $x=17 \mathrm{~cm}$ it appears at even smaller $q$.

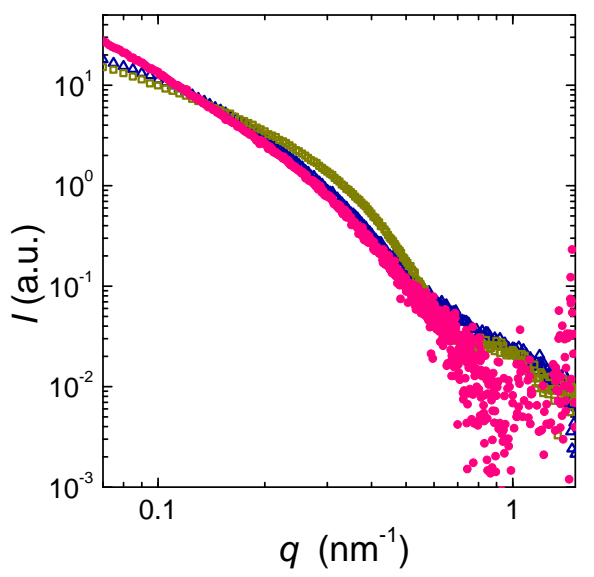

Figure 7.11: Comparison of equilibrium and in-flow low salt data of vimentin: The pink points are the SAXS signal recorded at $17 \mathrm{~cm}$ downstream at the conditions described in figure 7.10 , the green squares the scattering signal of vimentin wild type at $50 \mathrm{mM} \mathrm{KCl}$ and the blue triangles the scattering signal of the $Y 117 \mathrm{~L}$ mutant at $50 \mathrm{mM} \mathrm{KCl}$. The in flow curve is scaled to comparable units.

As the signal-to-noise ratio is once again in a range which allows comparisons to equilibrium data, we compare the scattering signal recorded at $x=17 \mathrm{~cm}$ (corresponding to a mean assembly time of about $6 \mathrm{~min}$ ) to that of the vimentin wild type and of the $Y 117 \mathrm{~L}$ mutant. As we have no equilibrium data recorded at $66 \mathrm{mM} \mathrm{KCl}$ which corresponds to the salt concentration in flow at $17 \mathrm{~cm}$ for either species we instead compare the data to the equilibrium curves recorded at $50 \mathrm{mM}$ $\mathrm{KCl}$ (figure 7.11). Obviously, the scattering curve from the wild type completely differs from that recorded in flow: The slope of the curves in the mid- $q$ range is quite different and the in flow curves show no indication of local minima and maxima around $0.7 \mathrm{~nm}^{-1}$ although the resolution would still be sufficient to distinguish them. The scattering data from the $Y 11^{7} \mathrm{~L}$ mutant resembles the long distance data much more: the slope of the curve in the mid- $q$ range is comparable. However, towards low $q$ the in-flow curve is considerably steeper. As the in-flow curve was recorded at a higher salt concentration than the equilibrium curve, this might at least partially be due to the salt concentration dependence of the filament structure, and therefore even at $17 \mathrm{~cm}$ downstream from the intersection we can conclude that we still see no concrete evidence for filament compaction.

Next, we consider how the scattering signal depends on the probe location in the pre-focusing geometry. At the end of section 6.1.3 we saw that for probe locations spaced twice as far apart as for the simple-cross geometry assembly times are similar but the salt gradient is significantly reduced. 
Figure 7.12 shows some exemplary scattering curves close to the intersection for flow rates comparable to the previously discussed cases $(850 \mu \mathrm{m} / \mathrm{s}(40 \mu \mathrm{l} / \mathrm{h})$ for the vimentin solution, $930 \mu \mathrm{m} / \mathrm{s}(80 \mu \mathrm{l} / \mathrm{h})$ for the salt buffer and $60 \mu \mathrm{m} / \mathrm{s}(5 \mu \mathrm{l} / \mathrm{h})$ for the low salt buffer), and a buffer salt concentration of $85 \mathrm{mM}$, resulting in an equilibrium salt concentration of about $65 \mathrm{mM}$. At the middle of the intersection (blue dots, $x=0$ ) we observe an overall up shift of the scattering and a steepening of the scattering curve. Going further downstream, the curves continue to steepen but the changes are small in comparison to the initial increase. Between $1 \mathrm{~mm}$ and $4 \mathrm{~mm}$ downstream there is nearly no change in the form of the curve. In the Kratky plot, we again observe the initial increase in peak height followed by the shift of the peak to smaller $q$ and a narrowing of the peak. For longer distances ( $1 \mathrm{~mm}$ and above), it appears that the low- $q$ flank of the peak could not be resolved.

In the pre-focusing geometry, in addition to data on the vimentin wild type, we also collected data on the $Y 11^{2} \mathrm{~L}$ mutant. Some exemplary scattering curves recorded at equivalent flow conditions and probe locations as above are given in figure 7.13. When comparing these data sets, it is important to note, that the background signal for the mutant data was larger as can be easily seen on in the case of the data recorded in the protein inlet which could only be resolved to about $0.2 \mathrm{~nm}^{-1}$, as opposed to $0.08 \mathrm{~nm}^{-1}$ for the wild type. Again, we can observe an overall upwards shift and steepening of the scattering curve upon contact with salt. However, in contrast to the wild type the scattering signal at low $q$ does not continue to increase after $x=100 \mu \mathrm{m}$ but stays at the same level. In addition to that, the decrease in the scattering signal at low $q$ remains and therefore the curvature of the curves increases. In the Kratky plot, we again see an increase of peak height and a shift of its location towards lower $q$ but even at $4 \mathrm{~mm}$ (corresponding to a mean assembly time of nearly $30 \mathrm{~s}$ ) we cannot identify narrowing.

As the data for the wild type and the mutant were recorded at identical conditions, we can directly compare them to each other as well as to equilibrium data. Figure 7.14a shows a direct comparison of the curves recorded at the center of the intersection. From about $0.3 \mathrm{~nm}^{-1}$ onwards, the two curves match well. Below that the curve from the mutant levels off towards smaller $q$ whereas the curve from the mutant continuous to increase resulting in an overall higher scattering. This means that at a mean salt concentration of about $20 \mathrm{mM}$ (see figure 6.14), a salt concentration where the equilibrium curves of the mutant and the wild type still resemble each other (see figure 4.9), there are significant differences in the scattering signal at short assembly times (about $4 \mathrm{~s}$ ), indicating differences in the assembly kinetics. At $4 \mathrm{~mm}$ downstream to the intersection (figure 7.14b), the curves again agree well 
(a)

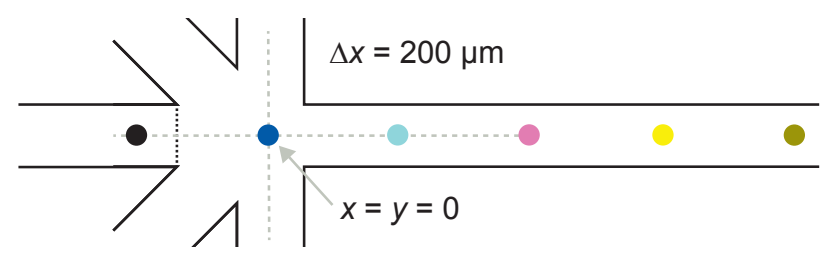

(b)

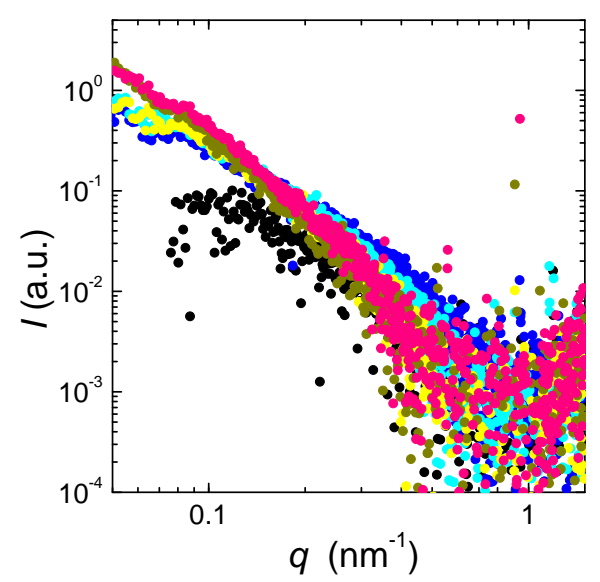

(c)

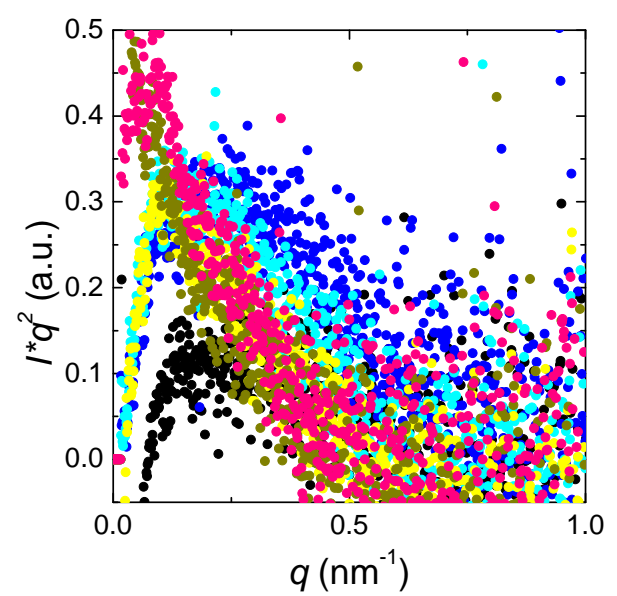

Figure 7.12: SAXS curves in microflow of vimentin wild type for the pre-focusing geometry with flow speeds of $850 \mu \mathrm{m} / \mathrm{s}(40 \mu \mathrm{l} / \mathrm{h})$ for the vimentin solution, a mean flow speed of the salt buffer solution $([\mathrm{KCl}]=85 \mathrm{mM})$ in the inlet of $930 \mu \mathrm{m} / \mathrm{s}(80 \mu \mathrm{l} / \mathrm{h})$ and a mean flow speed of the low salt buffer of $60 \mu \mathrm{m} / \mathrm{s}(5 \mu \mathrm{l} / \mathrm{h})$. (a) Localization of probe positions, the pink curves were taken at $x=4 \mathrm{~mm}$ outside of the sketch. (b) Exemplary SAXS signals near the intersection. (c) Corresponding Kratky plots. 
(a)

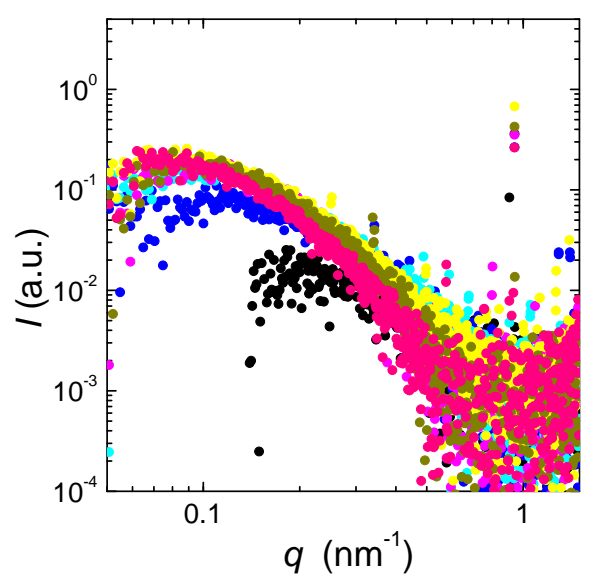

(b)

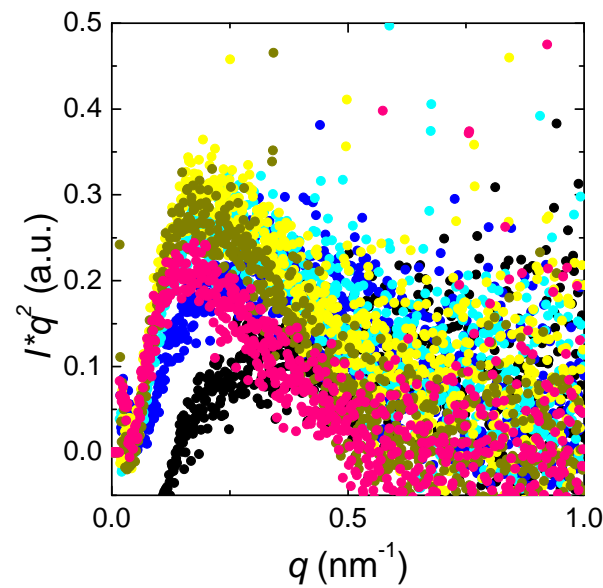

Figure 7.13: SAXS curves in microflow of the $Y 117 \mathrm{~L}$ mutant for the pre-focusing geometry with flow speeds of $850 \mu \mathrm{m} / \mathrm{s}(40 \mu \mathrm{l} / \mathrm{h})$ for the vimentin solution, a mean flow speed of the salt buffer solution $([\mathrm{KCl}]=85 \mathrm{mM})$ in the inlet of $930 \mu \mathrm{m} / \mathrm{s}(80 \mu \mathrm{l} / \mathrm{h})$ and a mean flow speed of the low salt buffer of $60 \mu \mathrm{m} / \mathrm{s}(5 \mu \mathrm{l} / \mathrm{h})$.(b) Exemplary SAXS signals near the intersection. (b) Corresponding Kratky plots. The colour code corresponds to figure $7.12 \mathrm{a}$, the pink curves were taken at $x=4 \mathrm{~mm}$.

(a)

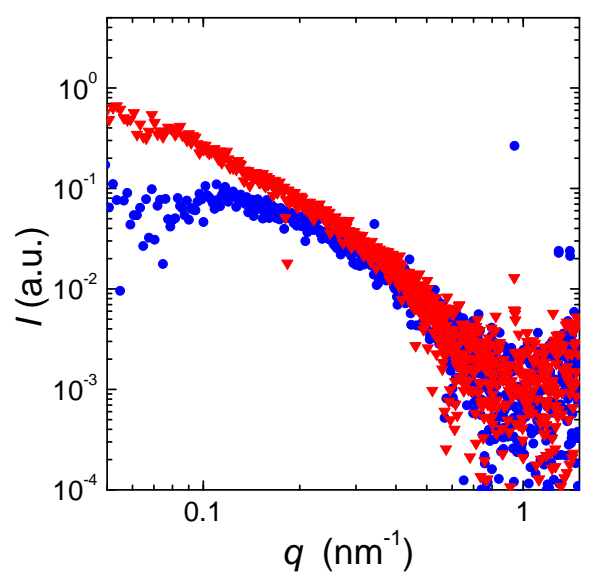

(b)

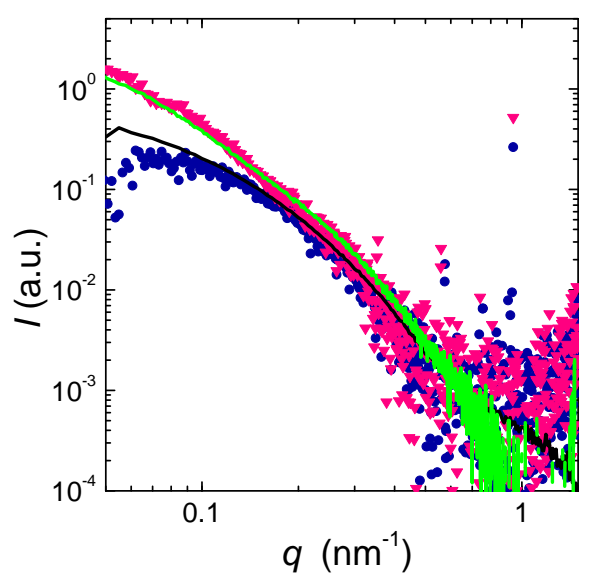

Figure 7.14: Comparison of the scattering curve of the wild type and the $Y 11^{7} \mathrm{~L}$ mutant of vimentin recorded at identical conditions. (a) At $x=0$. The blue circles represent the mutant and the red triangles the wild type. (b) At $x=4 \mathrm{~mm}$. The dark blue circles represent the mutant and the pink triangles the wild type. The black line is the equilibrium scattering data of the mutant at $50 \mathrm{mM} \mathrm{KCl}$ (scaled) and the green line stems from the simple cross geometry with flow conditions as described in figure 7.9 at $17 \mathrm{~cm}$ downstream. 
from about $0.2 \mathrm{~nm}^{-1}$ on but the differences below that value are stronger than in the middle of the intersection as the low $q$ scattering from the wild type changes less going downstream than that from the wild type. When we compare both curves to equilibrium data from the $Y 117 \mathrm{~L}$ mutant recorded at $50 \mathrm{mM} \mathrm{KCl}$ we see that the data from the mutant matches it very well and the data from the mutant deviates from it at lower $q$. Additionally, the comparison of the data at $4 \mathrm{~mm}$ to the curve recorded for the wild type in the simple cross geometry at $17 \mathrm{~cm}$ and a salt concentration of about $67 \mathrm{mM}$ (see figure 7.10) shows that the data at $4 \mathrm{~mm}$ from the wild type match it well, indicating that the wild type undergoes large structural changes on the very short timescale corresponding to the first few millimeters of flow in the pre-focusing setup and on very long timescales beyond those measured in microflow. To summarize, without any further analysis, we can already draw the following conclusions from our data:

- We can observe structural changes in microflow and these changes appear to depend on the flow conditions.

- The "long distance" data from the wild type at $160 \mathrm{mM}$ matches the equilibrium data from the $Y 117 \mathrm{~L}$ mutant at $100 \mathrm{mM}$ perfectly.

- At lower salt concentrations, no such match could be identified, but the curves resemble the scattering from the mutant more than that from the wild type.

- The wild type and the $Y 117 L$ mutant display systematic differences in the scattering already at low salt concentrations and assembly times.

- The scattering signal from the wild type remains constant, but different from the equilibrium state, over an extended period of time.

- The scattering from the mutant in a comparable time scale corresponds to that found in equilibrium at a lower salt concentration.

\subsection{Quantitative analysis}

Equipped with the above observations we can now aim to quantify the changes in the scattering curves and identify the scales in the microfluidic device on which they take place. As the changes in the curves are based on actual changes in the vimentin structure, these scales correspond to the scales on which the structure of vimentin changes. As we saw in section 6.1.3 the length scales in flow are actually related 
to time scales and salt concentrations and comparing the results from different flow conditions allows to some extent to distinguish between those two. Because the quantitative analysis requires a good signal-to-noise ratio both at low $q$ and at high $q$ and the most relevant changes in the signal occur close to the intersection, only the data from the device discussed in figure 7.1 will be further interpreted here.

A first, straightforward approach is to determine the cross-sectional radius of gyration for each measurement position via the Guinier approximation as introduced in sections 3.4.1 and 4.4.1. In figure 7.15 we see how the squared radius of gyration

(a)

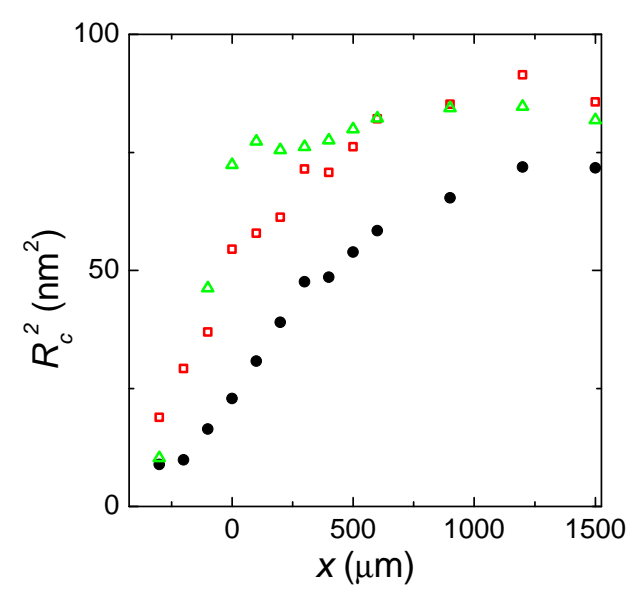

(b)

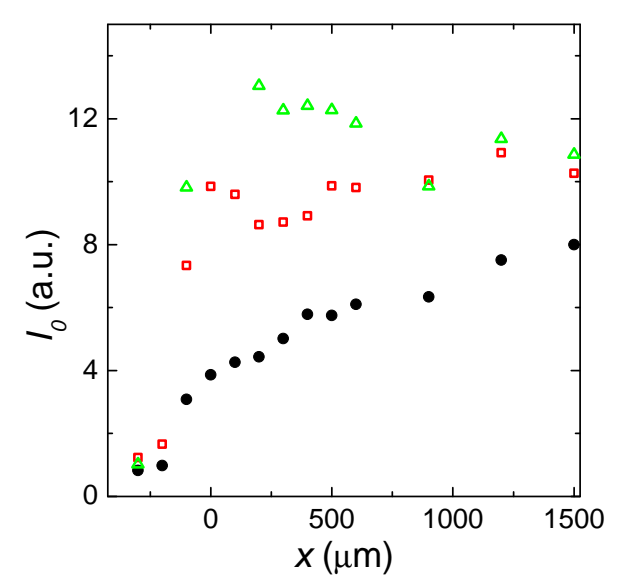

Figure 7.15: (a) Cross-sectional radius of gyration and (b) forward scattering amplitudes based on the Guinier approximation for different positions for flow as described in figure 7.1 (black circles), at flow rates reduced by a factor of 2 (red squares) and at flow rates reduced by a factor of 4 (green triangles).

and the forward scattering amplitude depend on the location in the device for three different flow speeds. We see that in all cases both values start to increase as soon as the vimentin enters the device (i.e. between $x=-200 \mu \mathrm{m}$ and $x=-100 \mu \mathrm{m}$ ) and that as the flow speed decreases this increase becomes more pronounced. The initial squared cross-sectional radius of gyration varies between the measurements and appears to lie at about $10 \mathrm{~nm}^{2}$ which is slightly higher than the value found for the equilibrium data (see figure 4.11a). This difference might be due to the changes in the scattering signal illustrated in figure 7.2 but also possibly due to the fact that in contrast to the equilibrium data the minimal value of $q$ put into consideration is not adjusted and hence the rather noisy data at low $q$ are also considered. Further downstream, all three curves level off to about $80 \mathrm{~nm}^{2}$, however the rate at which they approach this value differs, with the slowest flow increasing on the shortest 


\begin{tabular}{l|c|c|c} 
Mean flow speed & $770 \mu \mathrm{m} / \mathrm{s}$ & $390 \mu \mathrm{m} / \mathrm{s}$ & $190 \mu \mathrm{m} / \mathrm{s}$ \\
\hline Radius of gyration & $950 \mu \mathrm{m}$ & $350 \mu \mathrm{m}$ & $225 \mu \mathrm{m}$ \\
Forward scattering & $920 \mu \mathrm{m}$ & $150 \mu \mathrm{m}$ & - \\
1. derivate, mean & $350 \mu \mathrm{m}$ & $135 \mu \mathrm{m}$ & $120 \mu \mathrm{m}$ \\
1. derivate, variance & $700 \mu \mathrm{m}$ & $600 \mu \mathrm{m}$ & $500 \mu \mathrm{m}$ \\
2. derivate, mean & $650 \mu \mathrm{m}$ & $550 \mu \mathrm{m}$ & $500 \mu \mathrm{m}$ \\
2. derivate, variance & $450 \mu \mathrm{m}$ & - & - \\
PCA, 1. component & $320 \mu \mathrm{m}$ & - & \\
PCA, 2. component & - & - & $500 \mu \mathrm{m}$ \\
PCA, 3. component & $615 \mu \mathrm{m}$ & $515 \mu \mathrm{m}$ & $450 \mu \mathrm{m}$
\end{tabular}

Table 7.1: Comparison of decay lengths for different flow speeds based on different methods to characterize the changes in the scattering curves.

length scale. A squared cross-sectional radius of gyration of $80 \mathrm{~nm}^{2}$ is more than a factor of two higher than the largest equilibrium value observed for wild type vimentin without addition of magnesium $\left(25 \mathrm{~nm}^{2}\right.$ for $\left.100 \mathrm{mM} \mathrm{KCl}\right)$, but it is in the same range as the squared radius of gyration for the $Y 117 \mathrm{~L}$ mutant, which is $80 \mathrm{~nm}^{2}$ at $100 \mathrm{mM} \mathrm{KCl}$. As the scattering at longer distances is nearly identical to the one of the mutant at $100 \mathrm{mM} \mathrm{KCl}$ (see figure 7.4) this is not surprising. Table 7.1 gives the decay length for each set of flow speeds based on a simple exponential decay fit. We see that the length scale of the decay decreases with the overall flow speed from nearly $1 \mathrm{~mm}$ to less than $250 \mu \mathrm{m}$. When we compare the forward scattering amplitudes we observe similar trends: Before the intersection, the values for all three flow speeds are similar, as is their saturation level at long distances, which is about ten times the initial value. Again, the initial increase is quite strong and slower flow results in a steeper increase. However, in contrast to the radius of gyration the increase of forward scattering is non-monotonous for lower flow speeds with a peak at short distances. We cannot exclude that this local maximum stems from some minor aggregation of protein in the channel (which could not be seen in the microscope) but in this case actually the first point in the intersection should scatter the strongest (because aggregation usually seeds at the beginning of the intersection). Due to the non-monotony, for the slowest flow speed a single-exponential decay does not adequately describe the data at all, but for the medium flow speed the fit mostly ignores the peak and gives an average length scale (see table 7.1) which is shorter than that for the radius of gyration. For the fastest flow speed, the rate is nearly identical to the one found for the radius of gyration.

Should the short length-scale increase of the forward scattering be more than an artefact it would indeed indicate a behaviour which differs from what we observed 
in equilibrium for both the wild type and the mutant where both the cross-sectional radius of gyration and the forward scattering scale similarly with the salt concentration (see figures $4.12 \mathrm{a}$ and $4.13 \mathrm{c}$ ).

So far, we have only considered the low $q$ region relevant for the Guinier approximation to characterize the observed changes. However, as we saw in figure 7.1 the curves change over (nearly) the complete $q$ range and the changes are not necessarily coupled. The direct comparisons of two curves from the intersection and the end of the channel in figure 7.16a shows that in the mid- $q$ range the curvature of the SAXS curves in the double logarithmic plot changes: From clearly concave in the middle of the intersection to very slightly convex at the end of the device. Therefore, considering the first and second derivatives of the curves (i.e. $\frac{d \log I}{d \log q}$ and $\frac{d^{2} \log I}{d(\log q)^{2}}$ ) appears to be a promising approach. In order to reduce the effect of noise and to avoid issues due to negative data points we do not consider the direct derivatives but add a fixed offset and smooth the data before taking the logarithm. Then we interpolate the data with a spline at points with a fixed spacing in $\log q$. Figure 7.16a shows the resulting points used for estimating the derivatives in comparison to the original curves. Up to $0.3 \mathrm{~nm}^{-1}$ the differences are only minor but for $q$ values higher than that the differences become noticeable. Nevertheless, the main feature of interest, the shift from concave to convex persists.

In the first derivative based on this interpolation (figure $7.16 \mathrm{~b}$ ) we see that up to about $0.25 \mathrm{~nm}^{-1}$ the results from the center of the intersection and $2 \mathrm{~cm}$ downstream are parallel to the higher absolute value for the data recorded downstream (steeper curve). The curve from further downstream then turns upwards and crosses the curve from the intersection but constantly remains negative. The minimum of the curve at about $0.25 \mathrm{~nm}^{-1}$ confirms the existence of a turning point of the original SAXS curve in that region.

Continuing to the second derivative, we see, that as we would expect from the first derivate, it is basically identical for the two positions considered up to about $0.2 \mathrm{~nm}^{-1}$. Above that, both curves turn upward, with the curve from further downstream growing faster than the one from the intersection, crossing zero at about $0.25 \mathrm{~nm}^{-1}$.

In order to quantify the curves for each measurement position we need to reduce the number of values used to describe the curves. We choose the overall mean of the first derivative (general steepness of the curve), the variance of the first derivative between $0.27 \mathrm{~nm}^{-1}$ and $0.6 \mathrm{~nm}^{-1}$, which quantifies how much the slope varies in the mid- $q$ region, the mean of the second derivative between $0.2 \mathrm{~nm}^{-1}$ and $0.5 \mathrm{~nm}^{-1}$, 
(a)

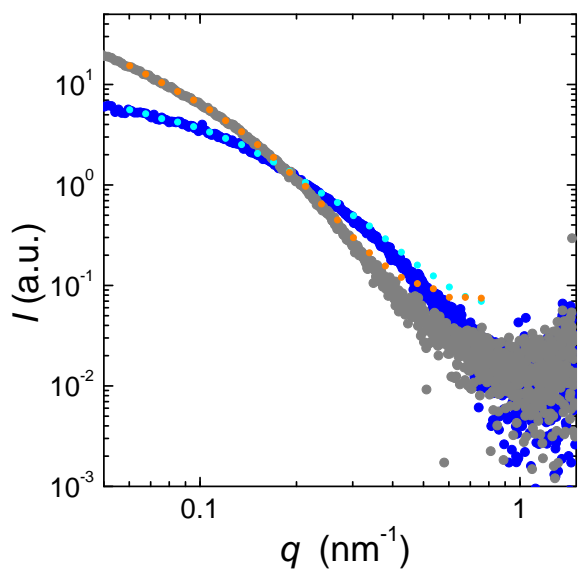

(b)

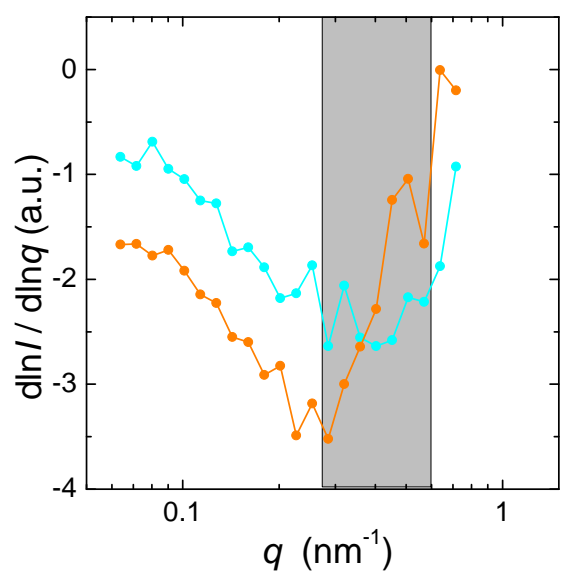

(c)

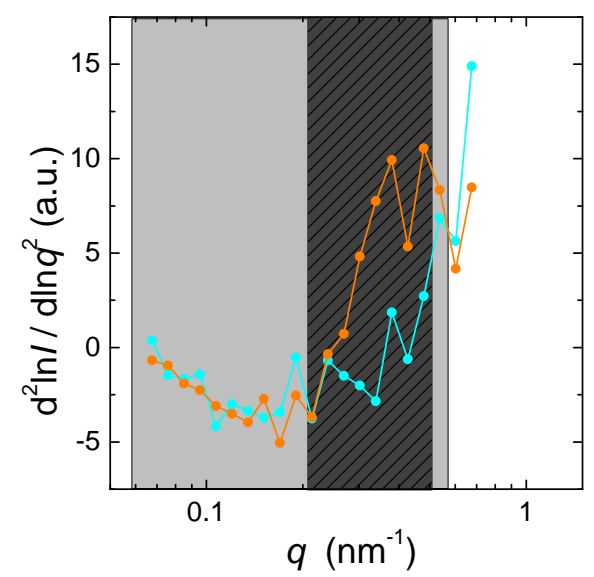

Figure 7.16: (a) Direct comparison of the SAXS curves in the middle of the intersection (blue) and at $2 \mathrm{~cm}$ downstream (gray). The light blue and orange circles indicate the points used for further analysis. They are equidistantly spaced over $\log q$ and the intensity is offset by 0.05 (a.u.). (b) First derivative of the curves. The light gray box indicates the region used to quantify changes in the variance of the derivative. (c) Second derivative of the curves. The light gray box indicates the region used to quantify changes in the variance of the derivative, the dark gray box the region on which the mean is based. 
where the curve in the intersection is considerably below that from further downstream, and the variance between $0.06 \mathrm{~nm}^{-1}$ and $0.6 \mathrm{~nm}^{-1}$, which is related to how much the curvature of the curve changes in different $q$-regions. Figure 7.17 a shows

(a)

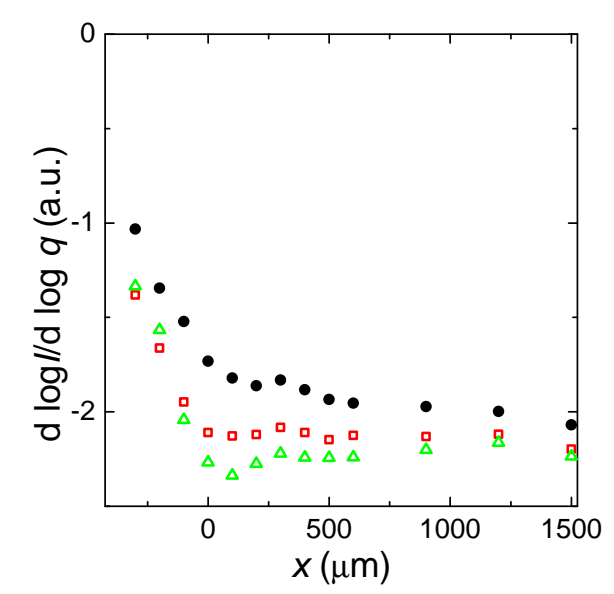

(b)

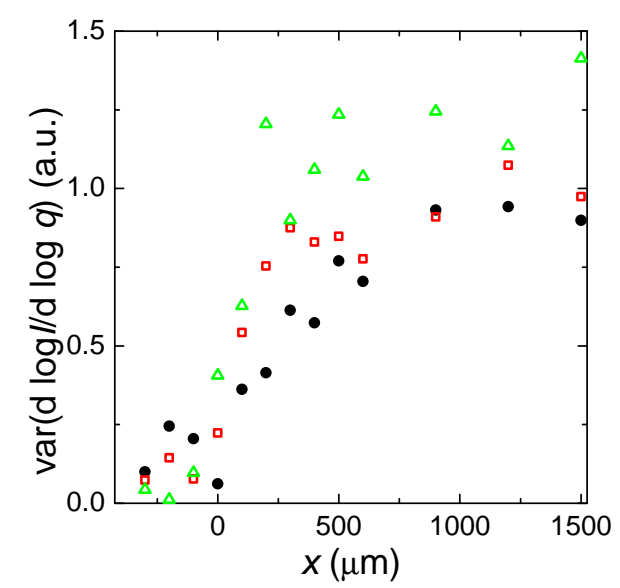

Figure 7.17: Quantification of the first derivative $\frac{d \log I}{d \log q}$ for different positions in the device for flow conditions as described in figure 7.1 (black circles), at flow rates reduced by a factor of 2 (red squares) and at flow rates reduced by a factor of 4 (green triangles). (a) Mean value of the derivative. (b) Variance of the derivative determined over the light gray region in figure $7.16 \mathrm{~b}$.

how the mean of the derivative depends on the position in flow at different overall flow speeds. As all curves are monotonously decreasing, the mean is always negative and as the scattering curves are steeper for probe locations further downstream, the mean of the derivative becomes smaller. Again, we observe some slight variation in the protein inlet values, and all curves converge to the same long distance value. Additionally, all three curves display a (local) minimum at $x=100 \mu \mathrm{m}$. As for the radius of gyration and the forward scattering amplitudes, thus convergence occurs on shorter length scales (see table 7.1). For the lower flow rates the resulting length scale is only slightly above the resolution of $100 \mu \mathrm{m}$, and for the fastest flow rate it is about a factor 2.5 shorter than the ones for the radius of gyration and forward scattering amplitudes.

In figure $7.17 \mathrm{~b}$ we see how the variance of the first derivative in the mid- $q$ region depends on the position in flow. The noise level is significantly higher and again all data sets begin and end at similar values with the distance over which the final values are reached being the shortest for the lowest flow speeds. The fact that the variance increases is indicative for the formation of the turning point, i.e. increased 
changes in the slope in the mid- $q$ region. Due to the noise level, no local extrema can be identified. Again, we can estimate the length scale for the increase with a single-exponential decay (results in table 7.1) and obtain values which are significantly larger than those obtained from the mean and decrease less with the flow speed than those from the Guinier approximation.

When we consider the second derivative (figure 7.18), we see that in the consid-

(a)

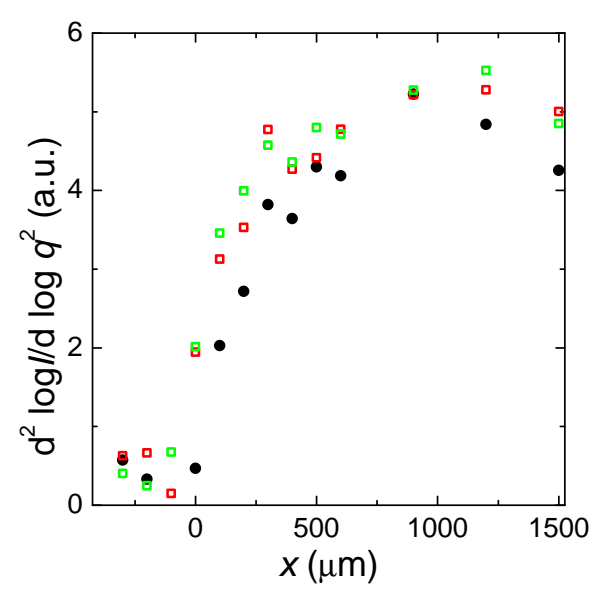

(b)

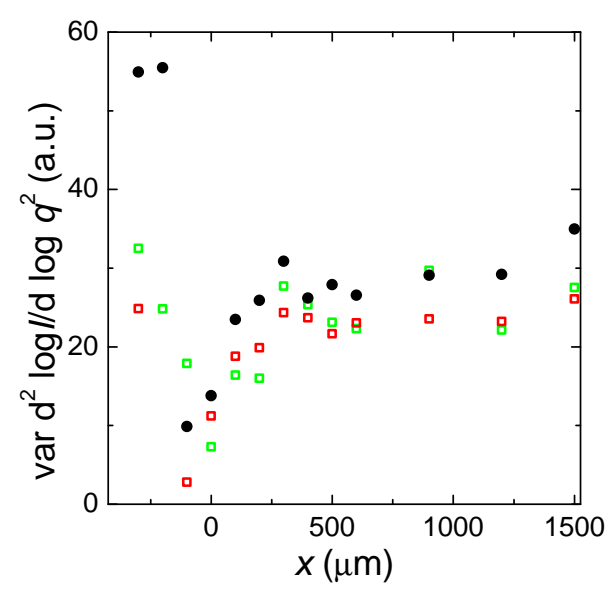

Figure 7.18: Quantification of the second derivative $\frac{d^{2} \log I}{d(\log q)^{2}}$ for different positions in the device for flow conditions as described in figure 7.1 (black circles), at flow rates reduced by a factor of 2 (red squares) and at flow rates reduced by a factor of 4 (green triangles). (a) Mean value of the derivative. (b) Variance if the derivative determined over the light gray region in figure $7.16 \mathrm{~b}$.

ered regions both the mean and the variance are very similar for all three curves. The mean in the mid- $q$ region is initially very close to zero and then increases to clearly positive values on a length scale of a bit more than $500 \mu \mathrm{m}$ (see table 7.1) and the length scale decreases only very slightly with the flow rate. The variance shows a behaviour which differs from what we saw so far: In the protein inlet it is rather high and decreases to nearly zero after entering the intersection. Then it increases again to about half the initial value. This curve form makes a singleexponential estimate of the relevant length scale difficult and only for the fastest flow speed an acceptable fit of the data can be performed with a resulting length scale which is comparable to the scale obtained from the mean of the first derivative.

Finally, we can quantify the changes in the curves more generally by principal component analysis (PCA). In PCA, one determines an orthogonal set of basis functions 
for the data ("components") in such a way that the first component describes the majority of the variability of the data (and hence corresponds to the mean). The subsequent components then account for the remaining variability. Due to this ordering usually very few components (in relation to the overall dimensionality of the data set) are necessary to adequately describe the data by linear compositions. The sign of both the components and the coefficients which describe the original data in PCA space ("scores") can in general be neglected [144]. As PCA evaluates a scattering curve in the context of all other scattering curves in the same set, it is over all sensitive to which set of curves is considered. As additionally physically relevant interpretation of the results is in general not possible, we refer to appendix E for details. The main result of the PCA is that three components suffice to characterize the changes in scattering curves. This can be considered an upper limit to the information content of each individual data set. Additionally for selected cases we can identify length scales which can be connected to the components and are listed in table 7.1 .

When we compare the individual length scales listed in table 7.1, we see that for each individual method the relevant length scale decreases with the flow speed. Additionally, we can identify re-occurring length scales: For the fastest flow speed $(770 \mu \mathrm{m} / \mathrm{s})$ they are about $900 \mu \mathrm{m}, 650 \mu \mathrm{m}$ and $350 \mu \mathrm{m}$, for the mid flow speed $(390 \mu \mathrm{m} / \mathrm{s}) 550 \mu \mathrm{m}, 350 \mu \mathrm{m}$ and $150 \mu \mathrm{m}$ and for the lowest flow speed $(190 \mu \mathrm{m} / \mathrm{s})$ $500 \mu \mathrm{m}, 250 \mu \mathrm{m}$ and $125 \mu \mathrm{m}$. Interestingly, the longest length scales for the two slower flow speeds are observed for the second derivative whereas the longest length scale for the fastest flow speed is obtained from the Guinier approximation.

\subsection{Interpretation}

The length scales for changes in the scattering signal we determined above (section 7.2) depend on the flow conditions, i.e. they are no intrinsic parameters of the vimentin self-assembly. For example, we cannot exclude that the changes in the length scales for the different flow speeds are only due to the fact that for different flow speeds identical probe locations correspond to different assembly times and salt concentrations. Therefore, it is necessary to connect the length scales to assembly time scales and salt concentration scales as these are the parameters determining the structure of vimentin. As we saw in chapter 6 , both the mean assembly time and the mean salt concentration increase monotonously with the $x$-position of the 
probe location. Because of this fact, we can plot different features of the scattering curves (e.g. radius of gyration) versus the mean assembly time or salt concentration and thereby study how these features depend on these parameters. It is, however, important to keep in mind that

- in salt concentration plots data points for one salt concentration correspond to different assembly times for different flow speeds and vice versa and

- identical mean salt concentrations (respectively assembly times) do not imply identical salt concentration distributions (respectively assembly time distributions).

In the following we consider the correlations between the squared cross-sectional radius of gyration $R_{c}^{2}$ and the mean assembly times and salt concentrations for the three different flow speeds discussed above. The other two relevant length scales for the changes of the scttering curves for each flow speed, which we previously determined, are discussed based on the mean of the first and second derivatives as introduced in figure 7.16 , respectively.

Figure $7.19 \mathrm{a}$ shows the correlation between the squared cross-sectional radius of

(a)

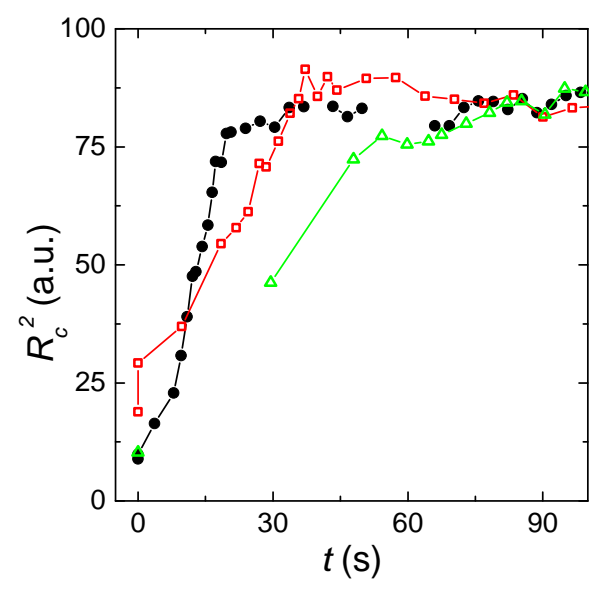

(b)

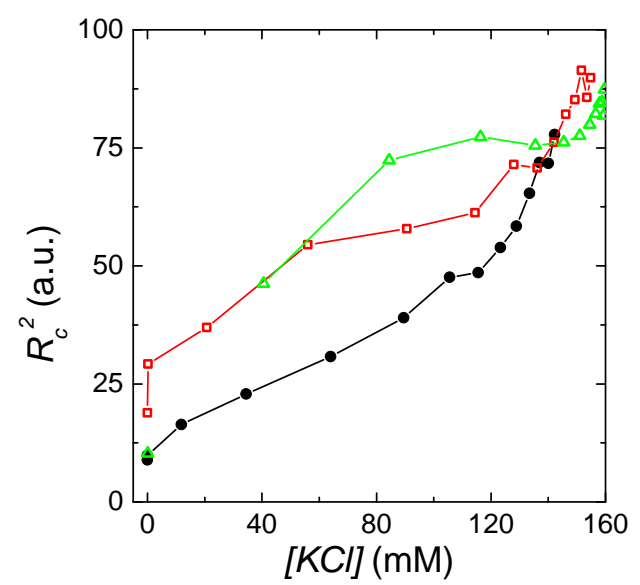

Figure 7.19: Dependence of the squared radius of gyration on (a) the mean assembly time and (b) the mean salt concentration for flow conditions as described in figure 7.1 (black circles), at flow rates reduced by a factor of 2 (red squares) and at flow rates reduced by a factor of 4 (green triangles).

gyration and the mean assembly times. For all three flow speeds $R_{c}^{2}$ increases with the assembly time and it appears that the increase is slower the lower the flow 


\begin{tabular}{l|c|c|c|c} 
flow speed & Method & length scale & time scale & {$[\mathrm{KCl}]$ scale } \\
\hline $770 \mu \mathrm{m} / \mathrm{s}$ & 2. derivative, mean & $650 \mu \mathrm{m}$ & $1.2 \mathrm{~s}$ & $80 \mathrm{mM}$ \\
$770 \mu \mathrm{m} / \mathrm{s}$ & Radius of gyration & $950 \mu \mathrm{m}$ & $1.5 \mathrm{~s}$ & $90 \mathrm{mM}$ \\
$770 \mu \mathrm{m} / \mathrm{s}$ & 1. derivative, mean & $350 \mu \mathrm{m}$ & $0.8 \mathrm{~s}$ & $40 \mathrm{mM}$ \\
$390 \mu \mathrm{m} / \mathrm{s}$ & 2. derivative, mean & $550 \mu \mathrm{m}$ & 2.3 & $80 \mathrm{mM}$ \\
$390 \mu \mathrm{m} / \mathrm{s}$ & Radius of gyration & $350 \mu \mathrm{m}$ & $1.5 \mathrm{~s}$ & $40 \mathrm{mM}$ \\
$390 \mu \mathrm{m} / \mathrm{s}$ & 1. derivative, mean & $150 \mu \mathrm{m}$ & $0.6 \mathrm{~s}$ & $5 \mathrm{mM}$ \\
$190 \mu \mathrm{m} / \mathrm{s}$ & 2. derivative, mean & $500 \mu \mathrm{m}$ & $5 \mathrm{~s}$ & $80 \mathrm{mM}$ \\
$190 \mu \mathrm{m} / \mathrm{s}$ & Radius of gyration & $250 \mu \mathrm{m}$ & $2.5 \mathrm{~s}$ & - \\
$190 \mu \mathrm{m} / \mathrm{s}$ & 1. derivative, mean & $125 \mu \mathrm{m}$ & $1.1 \mathrm{~s}$ & $15 \mathrm{mM}$
\end{tabular}

Table 7.2: Comparison of decay lengths for different flow speeds based on different methods to characterize the changes in the scattering curves. The time scales are based on single exponential fits and the salt concentrations scales represent the mean salt concentration at which the relevant observation values corresponds to the mean of the final and initial values.

speed. However, for the two faster flow speeds this difference is minor and for the lowest flow speed the temporal resolution at early times is too low for a conclusive statement. The corresponding time scales (based on a fit to a single exponential decay) given in table 7.2 are therefore mostly a rough idea.

Figure 7.19b shows the squared cross-sectional radius of gyration versus the mean salt concentrations. As the salt concentration has an upper limit of $160 \mathrm{mM}$, only data up to $1.5 \mathrm{~mm}$ downstream from the intersection are presented, because this region represents the majority of the changes and the mean salt concentrations are avaible directly from the simulations (see chapter 6). For all three flow speeds, the square of the radius of gyration reaches its final value of about $80 \mathrm{~nm}^{2}$ at $160 \mathrm{mM}$ $\mathrm{KCl}$. Below $120 \mathrm{mM} \mathrm{KCl}$, it seems that the lower the flow speed the higher the radius of gyration. A possible explanation for this observation is that at lower flow speeds the assembly time corresponding to a given mean salt concentration is always longer (see figure 6.9) and hence assembly would be further progressed, leading to larger aggregates and hence a larger radius of gyration. The differences in the salt concentration dependence of the radius of gyration can be quantified by estimating at which salt concentration the squared radius of gyration reaches $40 \mathrm{~nm}^{2}$, i.e. half of the final value. The results are given in table 7.2 .

Analogously, we can consider the correlations for the mean of the first derivative, see figure 7.20. Here, the dependence on the assembly appears very similar for all three flow speeds and the differences observed at early times for the lowest flow speed are likely due to insufficient temporal resolution. Again, the associated time scales are listed in table 7.2. When we plot the mean of the first derivative versus the mean 
(a)

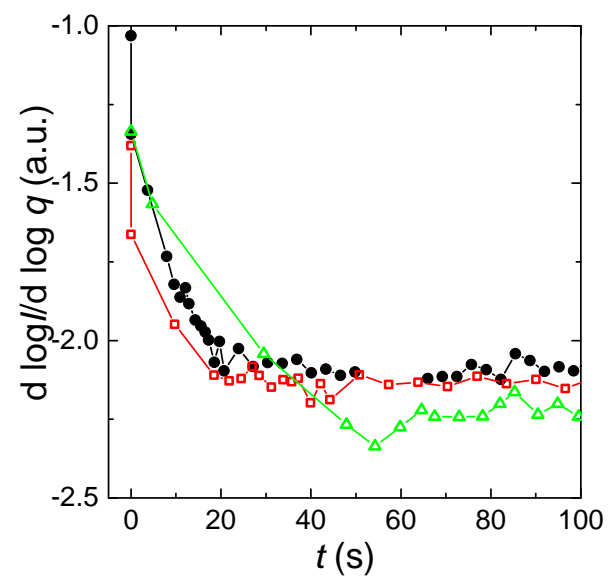

(b)

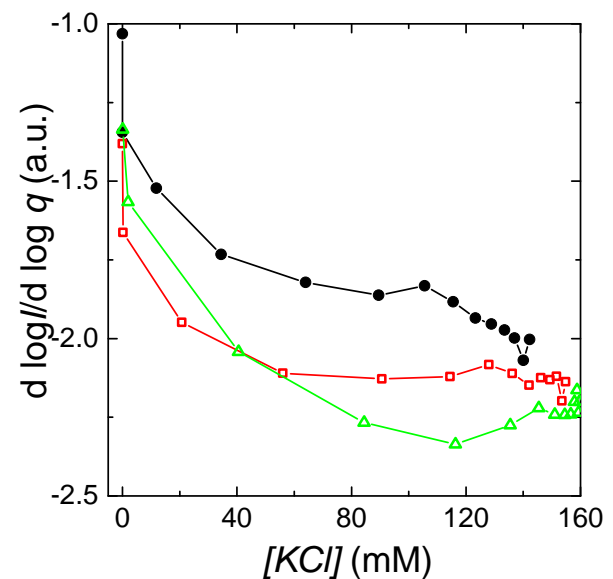

Figure 7.20: Dependence of the mean of the first derivative (see figure 7.16) on (a) the mean assembly time and (b) the mean salt concentration for flow conditions as described in figure 7.1 (black circles), at flow rates reduced by a factor of 2 (red squares) and at flow rates reduced by a factor of 4 (green triangles).

salt concentration it appears that for the lower flow speeds the value equilibrates at lower salt concentrations than for the fastest flow speed. As we already saw that mean first derivative-assembly time correlation does not seem to depend on the overall flow speed, this change in the salt concentration dependence is most likely again due to the fact that at lower flow speeds at identical salt concentrations the assembly time is longer and hence the change in the mean of the first derivative is further progressed. Table 7.2 gives the estimates for the salt concentration at which the middle between the initial and the final value of the mean of the first derivative is reached. The increase in the overall steepness of the double-logarithmic scattering curves is the fastest of the three length scales we observe and does not appear to depend on the salt concentration.

Finally, figure 7.21 gives the correlation plots for the mean of the second derivative, i.e. the measure of the curvature in the mid $q$-range. Here, the increase in the mean curvature becomes significantly slower for lower flow speeds with a difference of more than a factor of three in the relevant time scale (see table 7.2). This strong difference is also the reason why the analysis method which provides the longest length scale in the previous analysis is different for the different flow rates (see table 7.1). Also, the change in the curvature for the lowest flow speed possesses the slowest overall time constant we can identify ( $5 \mathrm{~s}$ ). Even more interestingly, the three curves in the mean second derivative versus salt concentration plot fall above each other. This implies 
(a)

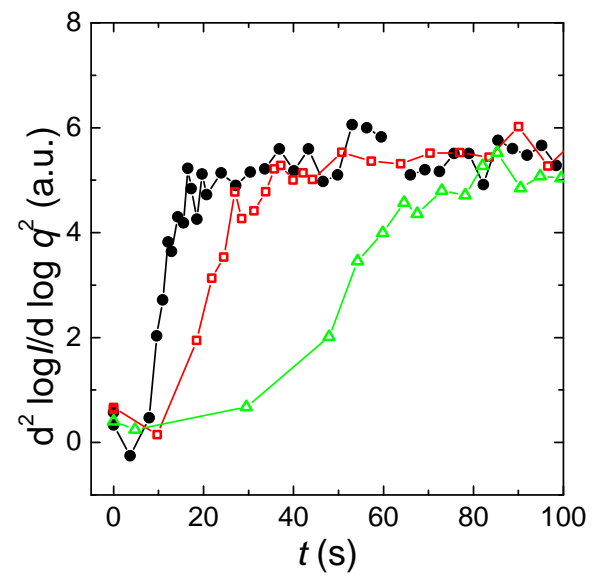

(b)

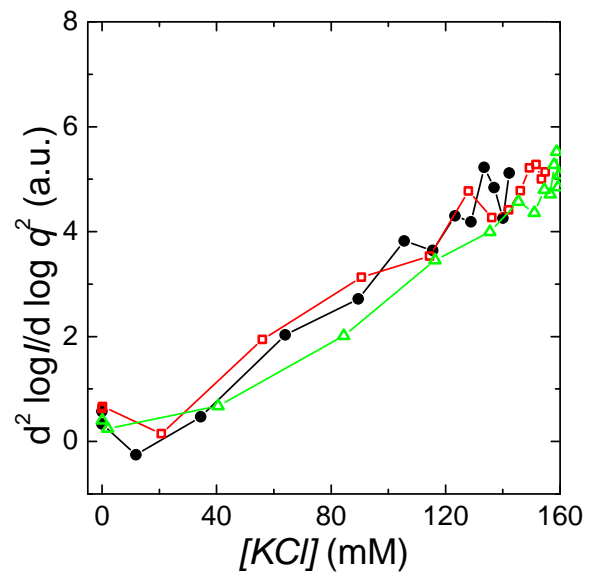

Figure 7.21: Dependence of the mean of the second derivative (see figure 7.16) on (a) the mean assembly time and (b) the mean salt concentration for flow conditions as described in figure 7.1 (black circles), at flow rates reduced by a factor of 2 (red squares) and at flow rates reduced by a factor of 4 (green triangles).

that this structural feature does not depend on the assembly time but only on the local salt concentration. Additionally, the curves appear linear, indicating that the second derivative would continue to increase with higher salt concentration. Again, the typical salt concentration associated with the change in this value is listed in table 7.2 .

To summarize we see that

- the radius of gyration appears to depend on both the assembly time and the local salt concentration

- the mean steepness of the curve in the double-logarithmic plot seems to depend only on the assembly time and

- the mean curvature in the mid- $q$ range in the double logarithmic plot appears to depend only on the salt concentration.

How can we understand these findings? We saw above that the "final" curve in flow (at $160 \mathrm{mM} \mathrm{KCl}$ ) corresponds to that of the $Y 117 \mathrm{~L}$ mutant at $100 \mathrm{mM}$. When we consider how the scattering curve of the mutant protein depends on the salt concentration (see figure 4.7) we see that whereas the scattering amplitude at high $q$ remains roughly constant, the scattering amplitude at low $q$ raises strongly from low salt conditions to $100 \mathrm{mM} \mathrm{KCl}$, resulting in an overall increase in slope. However, 
most of this increase occurs below $50 \mathrm{mM} \mathrm{KCl}$ and further analysis shows that it coincides with a decrease in the amount of tetrameric vimentin (see figure 4.21c). Therefore, one possible interpretation is that the increase in the mean of the first derivative corresponds to the assembly of vimentin into larger aggregates and thereby the reduction of the free tetramer pool. This process is therefore rather fast (time constant $1 \mathrm{~s}$ ) and in our setup likely to be to some extent limited by the mixing of salt into the protein jet, as we know from chapter 4 and the literature [43] that at low salt concentrations (below $20 \mathrm{mM}$ ) a significant amount of vimentin remains tetrameric.

As the change of curvature seems to depend only on the salt concentration it is reasonable to directly correlate it to the changes in curvature observed for the mutant protein. In section 4.4 we postulated that the curvature of the scattering curve of the mutant is related to the ratio of scattering from the side chains of an ULF to the core, $b$, such that a higher value for $b$ corresponds to an more S-like shape. In terms of the analysis we apply here, hence a larger $b$ equals a larger value for the mean of the second derivative. The linear dependence of the curvature on the salt concentration is an additional indication that after the "fast" processes (i.e. increase of overall steepness and radius of gyration) are completed, the structure of the vimentin aggregate depends only on the local salt concentration as $b$ also linearly depends on the salt concentration. It is, however, important to remember that scattering from wild type vimentin in flow does not correspond to that of $Y 117 L$ at identical local salt concentrations but rather to that found at lower salt concentrations (see figures 7.4 and 7.11) whereas the scattering from the mutant in flow does seem identical to that in equilibrium for identical salt concentrations (see figure 7.14).

\section{Comparison to the literature}

How do our results compare to previous studies on the kinetics of vimentin assembly? Our most obvious result is that we see no indication of compaction: Even at long assembly times $(6 \mathrm{~min})$ the scattering signal resembles the signal from the non-compacting $Y 117 L$ mutant and not the one from the wild type (see figure 7.11). TEM studies and near-UV circular dichroism studies (the latter in solution) give a time constant for compaction of $13 \mathrm{~min}$ in "TRIS" buffer and for "phosphate" buffer faster compaction on the time scales of seconds has been reported $[27,44,46,47]$. The fact that we see no change in the scattering signal between $30 \mathrm{~s}$ and $6 \mathrm{~min}$ (see figure 7.14b) and that the scattering curves are similar to equilibrium curves 
of the mutant protein with radii that are larger than that of the equilibrium state of the wild type protein, however, indicates that we see no compaction to mature filaments on the timescale of a few minutes. Additionally, on shorter time scales (figures 7.19 and 7.20) the changes in the scattering curve indicate growth, but not compaction, excluding compaction on the short time scales reported for "phosphate" buffer. Therefore we can conclude that, in contrast to previous findings, we see no indication of compaction up to $6 \mathrm{~min}$. It is, however, important to note, that the diameter of $Y 117 \mathrm{~L}$ in "phosphate" buffer is considerably smaller than the one of wild type protein ULFs in "TRIS" buffer: $13 \mathrm{~nm}$ opposed to $17 \mathrm{~nm}$. Hence the visible effect of compaction in micrographs is limited. On longer timescales $(24 \mathrm{~h})$ however, the filament radius does reduce (see chapter 4).

As even for the fastest set of flow speeds we discussed here the temporal resolution lies in the range of seconds, it should be impossible to access lateral assembly dynamics (except for the difference between the protein inlet and the first measurement point in the intersection) because at the protein concentration we employ for our experiments it proceeds on a sub-second timescale, see figure 6.21. Instead, we can observe a change in the cross-sectional structure on longer time scales which appears to be directly connected to the local salt concentration, see figure 7.21. Elongation (by addition of single ULFs) is somewhat slower. We can estimate an upper limit for the associated rate to $4.25 \mathrm{~s}^{-1}$ from the literature value of $1.7 \mu \mathrm{M}^{-1} \mathrm{~s}^{-1}$ based on the maximum possible concentration of ULFs. Figure 6.21 shows that the lateral assembly rates found in the literature result in temporal range for elongation of about a second and it is possible (due to the reasons discussed above, see figure 7.14) that the fast change we observe in the slope of the scattering curve of the wild type is due to elongation. However, as our temporal resolution is limited, we can only note that the estimate for the rate of the rise in steepness of about $1 \mathrm{~s}^{-1}$ is on the correct order of magnitude.

\section{Implications for assembly models}

How do our findings fit into the current model for vimentin filament self-assembly? We could directly confirm that the equilibrium structure formed by the $Y 117 \mathrm{~L} \mathrm{mu-}$ tant does indeed correspond to an intermediate of the assembly process as illustrated in figure 7.4. In addition, we can identify a dependence of the structure of the intermediate state on the local salt concentration. However, the structure of the intermediate state seems to correspond to the structure of the mutant protein at a 
slightly lower salt concentration, which based on our analysis in chapter 4 implies that the intermediate state has a smaller diameter than the mutant at identical salt concentration.

So far, it has been postulated that the fact that part of the coiled-coil structure of $Y 11^{7} \mathrm{~L}$ is more stable than for the wild type protein inhibits elongation and compaction [45]. In addition, our findings in chapter 4 also indicate that the structure of the tail domain changes between ULFs and mature filaments: We see that both the radius of gyration and the scattering intensity we associate with the tails are larger for the mutant protein. Also, in electron micrographs, compacted filaments tend to appear smoother than their precursors, also indicating a possible reduction of the tail corona. However, filament formation, albeit with a less well defined mass per length, is still possible even for tail-less vimentin $[27,145]$. This indicates that interactions of the tail domain with the rod might be involved in the elongation or compaction process but are not instrumental. 


\section{Chapter 8}

\section{Discussion of biophysical context}

Having discussed the implications of our results on vimentin structure and assembly in chapters 4 and 7 , we now consider some aspects of vimentin self-assembly in a broader (bio-) physical context.

\subsection{Meta-stability of the ULF state}

In chapter 7 , we saw that the pre-compaction intermediate state is surprisingly stable with a life time of at least several minutes, possibly even hours. But what are the reasons for this long life time? First of all, we can exlude two possible mechanisms, which slow down other multi-component reactions:

1. High number of reactants required: For systems in which the equilibrium state is built up from a large number of individual building blocks and no stable intermediates exists, meta-stable states with a life-time up to weeks can be observed [146]. Albeit the number of vimentin monomers involved in the compaction is not small (32 monomers), they are already located close to each other in the pre-compacted filament.

2. Nucleation: The formation of a reaction nucleus as the rate limiting step, as observed for example for actin polymerization [1] or crystallization of colloidal particles [147], is to some extent a variation of the previous scenario. As the local vimentin concentration in a pre-compacted filament does not vary tremendously, this scenario can also be excluded.

In the following, we present some considerations on which factors might instead contribute to the slowing down of compaction kinetics. 


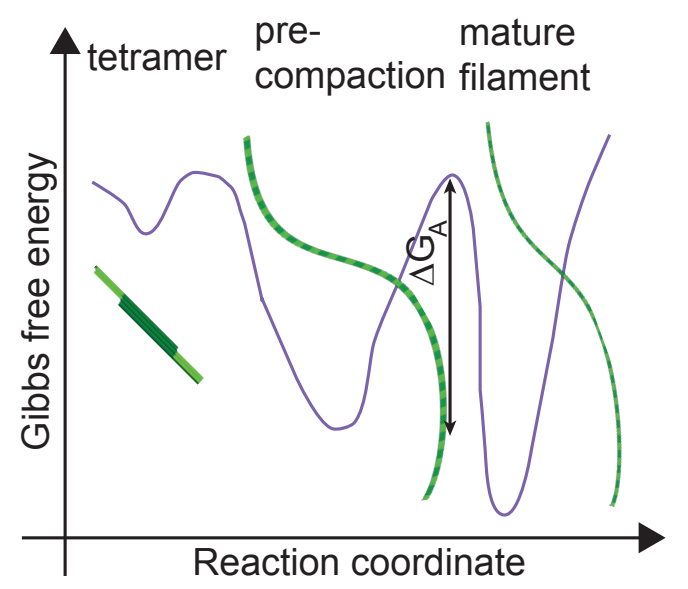

Figure 8.1: Sketch of the suggestion for the Gibbs free energy of the vimentin assembly including the meta-stable precompaction state in dependence of some reaction coordinate (e.g. local tetramer density).
The fact that mature filaments are very stable structures and that compaction seems basically irreversible at a fixed salt concentration [21], indicates that they are energetically favorable but that the energy barrier separating un-compacted and mature filaments is relatively high. Figure 8.1 sketches a projection of an energy landscape for the lateral processes (i.e. lateral growth to ULFs and compaction) illustrating these features. In the following paragraphs, we will explain why we propose such a shape for the landscape. The landscape possesses three local minima, corresponding to free tetramers and filaments pre-compaction, and a global minimum corresponding to mature filaments. The reaction coordinate, i.e. the coordinate used to describe the conformation state of the vimentin filament, is somewhat arbitrary. For example it could be related to the mean local tetramer density (i.e. the inverse of the mean distance between tetramers). Elongation is missing in this sketch, assuming that the relevant reaction coordinates are orthogonal to the one illustrated here. In order to account for the comparably fast and apparently uni-directional formation of ULFs, the activation energy from the tetrameric to the pre-compaction state is rather low and the energy barrier is considerably higher for the inverse reaction. Proceeding from pre-compacted to mature filaments, the energy barrier is high in both directions: Reaction rates for both compaction and de-compaction are low, resulting in a metastable pre-compaction intermediate state (at the second local minimum) and a very stable mature filament state.

So far, the compaction scenario shares some similarity with two-state protein folding when we equate the pre-compaction state to the unfolded state and the mature filament state to the folded state [148]:

- Two clearly separate states can be identified.

- The initial state is meta-stable, i.e. an energy barrier exists. 
- The conformation space for the initial state is considerably larger than the one for the final state.

- The reactants (individual amino acids for folding / vimentin monomers for compaction) are geometrically confined with respect to each other.

However, there are also distinct differences, implying that vimentin compaction is a more complex scenario:

- When considering that filament formation is a quintary protein structure formation processes (tertiary structure $=$ vimentin monomer, quaternary structure $=$ vimentin tetramer $)$, it is likely that the lower level quaternary structure and even tertiary structure are re-arranged during the compaction step [46]. For two-state folders, lower level re-arrangement would correspond to changes in the primary structure and therefore does no occur.

- The number of amino acids whose structural conformation can change during the compaction process is considerably larger than the number of amino acids typically involved in protein folding.

- Existing secondary, tertiary and quaternary structure additionally confines the configuration space accessible to individual amino acids in the case of vimentin compaction.

A theoretical model for the reaction rate for unimolecular reactions such as protein folding is given by

$$
k_{f}=\xi^{-1} e^{-\frac{\Delta G_{A}}{k_{b} T}}
$$

where $\xi$ is the (local) friction, whose physical origin is not specified, $\Delta G_{A}$ the necessary activation energy (see figure 8.1) and $k_{b} T$ the thermal energy $[149,150]$. Hence there are two factors which can slow down a reaction: a high activation energy and high local friction.

When we try to understand why compaction is slow, a first, simple argument is that of packing. On the one hand, we can estimate the cross-sectional area of mature filaments $A_{F} \lesssim \pi(5 \mathrm{~nm})^{2}=\pi 25 \mathrm{~nm}^{2}$, see section 2.1. On the other hand, the cross-sectional area of an individual tetramer can be estimated via $A_{T} \gtrsim$ $\pi(2 \mathrm{~nm})^{2}=\pi 4 \mathrm{~nm}^{2}$. For eight tetramers in the filament cross-section, this would result in a cross-sectional area of $\pi 32 \mathrm{~nm}^{2}>\pi 25 \mathrm{~nm}^{2}$. This implies that the ordering of the subunits (monomers, dimers or tetramers) in mature filaments is more crowded than in tetramers and that the available conformational space becomes 
reduced by compaction. This reduction in the conformational space is illustrated in figure 8.1 by a reduced width of the mature filament minimum of the energy landscape as opposed to the pre-compaction filament minimum. Due to the rather tight packing of the final state, it is possible that there are necessary transition states with an even smaller configuration space. These states would have only little entropy resulting in a larger activation energy and hence slow kinetics. In figure 8.1 such states would contribute to the local energy maximum between pre-compaction and mature filaments.

An additional factor to consider is that the results by Georgakopolou et al. indicate structural changes in the vimentin coils during compaction [46]. As the coil is a rather inflexible structure the local friction (due to energetical confinement in the structure) for the involved amino acids is likely to be relatively large. According to equation 8.1, this also leads to slower reaction rates.

\subsection{Comparison to other biopolymer systems}

How can we interprete our results in the broader context of the interaction of rodlike polyelectrolytes with ions? A recurring theme is the formation of larger aggregates with a well defined structure (i.e. bundling): For microtubules small di-valent cations (e.g. calcium at about $40 \mathrm{mM}$ ) have been reported to induce bundle formation with a necklace like bundle topology. Interestingly, no bundling was found for magnesium chloride or monovalent ions [151]. For F-actin, magnesium ions at concentrations above $10 \mathrm{mM}$ have been shown to induce the formation of bundles of about five to seven filaments [152]. Also, for vimentin filaments, hints for bundling by proteins such as smooth muscle calponin have been reported [153]. Additionally, rheology and TEM experiments on vimentin assembled in the presence of both sodium and magnesium ions indicate the formation of cross-linked filament networks [38-40]. When magnesium ions are added to vimentin filaments formed in the presence of potassium chloride, at magnesium concentration above about $10 \mathrm{mM}$ the vimentin networks contract [41].

Interestingly, the SAXS signals of bundles both microtubules and F-actins induced by di-valent cations display characteristic peaks $[151,154]$. In chapter 4 we saw, that the SAXS signal of vimentin filaments in the presence of di-valent ions shows no peaks at all, indicating that whereas magnesium might cross-link vimentin filaments, it does not appear to induce bundling. In the following, we cannot expect to observe bundling in our experiments. 


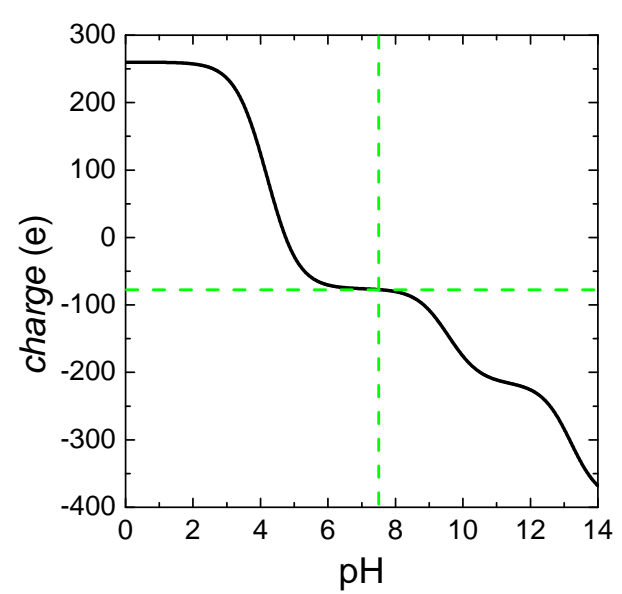

Figure 8.2: Estimate of the effective charge of a vimentin tetramer in dependence of the $\mathrm{pH}$ based on the $\mathrm{pk}_{a}$ values of the individual amino acids [54]. At $\mathrm{pH}=7.5$ the excess charge is $-77 \mathrm{e}$, as marked by the cross.
We need to keep in mind that in our experiments, we are not considering the influence of ions on the super-structure of filaments but rather the influence of ions on the super-structure of vimentin tetramers (due to the fact that all ion species are added simultaneously). Therefore, instead of comparing vimentin filaments to microtubules, F-actin or other polyelectrolytes we need to compare vimentin tetramers to these systems. One important parameter to describe a polyelectrolyte is its effective charge density. For the vimentin tetramer, we can estimate the number of excess charges based on its amino acid sequence and typical $\mathrm{pk}_{a}$ values of the acidic and basic amino acids $[54,155]$. Figure 8.2 shows the re-

sulting titration curve and we see that at a $\mathrm{pH}$ value of 7.5 , the effective charge has a plateau at -77 e. In combination with the tetramer length of $60 \mathrm{~nm}$, this results in a charge density along the tetramer of about $a=-1.25 \mathrm{e} / \mathrm{nm}$. For a filament with a cross-section of eight tetramers, this basic estimates gives about $-14.5 \mathrm{e} / \mathrm{nm}$, as the length per tetramer reduces to $43 \mathrm{~nm}$ [48]. In comparison, the absolute value of the (negative) linear charge density of DNA at neutral $\mathrm{pH}$ is about $5.9 \mathrm{e} / \mathrm{nm}$, the one of F-actin is about $4 \mathrm{e} / \mathrm{nm}$, and the one for microtubules is $2.5 \mathrm{e} / \mathrm{nm}[156,157]$, i.e. all three polymers have a charge density larger than that of vimentin tetramers. In the theory of counterion condensation, the charge density is described in terms of the charge density parameter $\zeta=\lambda_{b} a$ where $\lambda_{b}=e^{2} / 4 \pi \epsilon_{0} \epsilon k_{b} T \approx 0.71 \mathrm{~nm}$ is the Bjerrum length, the distance at which the electrostatic interaction energy equals the thermal energy [158]. Following from the above, vimentin tetramers have the smallest $\zeta$ (0.88), followed by microtubules (1.8), F-actin (2.8) and DNA (4.2). We see that only for vimentin tetramers $\zeta<1$. For ions with a charge $Z$ the Manning counterion condensation theory predicts that the fraction $\Theta$ of the excess charge that becomes condensed is

$$
\Theta=\left(1-\frac{1}{Z \zeta}\right)
$$


For DNA, it has been estimated that $\Theta>0.9$ is necessary for condensation [159]. As many di-valent cation species can induce F-actin bundling, but not necessarily microtubule bundling, the critical $\Theta$ for bundling appears to lie in the range of 0.75 to 0.8 [159]. For vimentin tetramers, this implies a minimum cation charge of $Z=4$, which is not given in the systems we studied.

From the above considerations, it is clear that the physical basics of the assembly of vimentin filaments from tetramers are different from those that induce bundling of F-actin or microtubules. Hence, it is not surprising that, whereas di-valent cation induced bundles of both microtubules and F-actin display characteristic peaks, no peaks are observed for vimentin filaments formed in the presence of di-valent ions. Finally, we note that most of the above considerations are based on the charge density of tetramers. For vimentin filaments, the charge density based on the above estimate is much higher and hence 8.2 does not contradict the formation of a divalent ion condensation shell for filaments assembled in the presence of magnesium, as suggested in section 4.5. Additionally, based on our above estimate, the linear charge density of vimentin filaments might indeed be high enough to allow bundling upon the addition of cations, as long as the filaments remain trapped in their original structure [41]. 


\section{Chapter 9}

\section{Conclusions}

In the previous chapters, we investigated how the structure of vimentin filaments depends on the assembly conditions, i.e. salt concentrations and assembly time. Here, we summarize our most important findings and their implications. In the first part of this work, we investigated how the equilibrium structure of vimentin, i.e. the structure more than $24 \mathrm{~h}$ after initiation of assembly by the addition of ions, depends on the composition of the ionic environment by small angle X-ray scattering:

- We see that the equilibrium structure of the vimentin wild type for different ionic environments (both filament forming and not filament forming) does not depend on the total protein concentration. In contrast, the structure of aggregates of the vimentin mutant $Y 117 \mathrm{~L}$, which is trapped in a pre-elongation state, at filament forming conditions depends on the overall protein concentration. This indicates that at sufficiently high protein concentrations, the mutant protein might acutally elongate.

- The diameter of both the vimentin wild type and the Y117L mutant protein slightly increases with increasing potassium chloride concentration. However, the diameter of the mutant is generally larger and the contributions of the tail regions also appear to contribute more strongly to the scattering signal, indicating an involvement in the formation of the filament structure beyond the ULF stage.

- The addition of magnesium chloride (di-valent cations) instead of potassium chloride (mono-valent cations) results in filaments with a larger diameter (up to a factor of nearly two). Additionally, both the apparent size of the tail regions and their contribution to scattering increase significantly, indicating a 
possible increase of the magnesium ion concentration in the tail region. When potassium chloride is present, these effects are less pronounced.

In the second part of this work, we designed microfluidic devices for controlled mixing of ions into a flowing vimentin solution. This device allows us to access structural changes due to differences in the local salt concentration on the time-scale of seconds in situ with SAXS:

- We could directly proof that the equilibrium structure of the Y117L mutant protein corresponds to that of an intermediate state of the assembly of the vimentin wild type.

- In addition, the structure of the intermediate state at a certain salt concentration corresponds to the structure of the mutant protein at a slightly lower salt concentration. This implies that the $Y 117 \mathrm{~L}$ mutant protein is indeed a good model for intermediate stages of vimentin assembly.

- The intermediate state appears to have a life-time in the range of several minutes, i.e. it is very stable.

- At salt concentrations where the scattering signal of the equilibrium state of the vimentin wild type and the $Y 117 \mathrm{~L}$ mutant is nearly identical, the scattering signal on the timescale of seconds differs. This is indicative for different assembly speeds of the two proteins or possibly even a stop of assembly for the mutant protein.

The above leads to the conclusion, that the $Y 117 \mathrm{~L}$ mutant is a good model for the pre-elongation intermediate of vimentin, but not necessarily for the investigation of reaction kinetics. We could also show, that the cross-sectional structure of both vimentin filaments and pre-elongation intermediates depends on the cation concentration and that the pre-elongation intermediate adapts its structure due to salt concentration changes on a sub-second timescale. 


\section{Chapter 10}

\section{Outlook}

In this work, we could show that SAXS is a tool well suited to study the dependence of intermediate filament structure on the ionic environment. We could address a number of issues which result in new, interesting questions:

- Does the vimentin assembled in micro-channels actually progress to mature filaments as we observed them in the capillary experiments on longer time scales? This question can be addressed by collecting the outflow from a microfluidic device, and investigating it with SAXS and TEM after appropriate assembly times.

- What happens on shorter (sub-second) assembly times? In principle, this question can be addressed by decreasing the size of the protein jet in the inflow experiments by increasing the relative flow speed of the buffer. However, for most currently available $\mu$-SAXS setups, the width of protein jet then becomes smaller than the X-ray probe, which results in a decrease of the signal in comparison to the background scattering from the buffer and the device material. As the probe size at $\mu$-SAXS setups continues to decrease, further development in microfluidic devices for SAXS might render this question experimentally accessible in the near future.

- Is the observed increase in the scattering of the tail region of vimentin filaemts in the presence of magnesium indeed due to an excess concentration of the magnesium in the tail region? There are several possible approaches to this question. For example, one could replace the magnesium with calcium or zinc which have the same ionic valency but different mass. This should result in an even further increase in scattering from the tail region while the scattering from 
the rod should remain mostly unaffected. Using zinc also adds the possibility to study the ion distribution by anomalous scattering (i.e. scattering above and below the absorption edge), as zinc is considerably heavier than the remaining elements in the sample.

- Is the vimentin tail region re-organized during the maturation from ULFs to mature filaments? This question might be addressed by recording both the equilibrium and in-flow intermediate state of the tailless vimentin mutant and comparing it to both wild type protein and the $Y 117 \mathrm{~L}$ mutant.

- Does the structure of the vimentin filaments differ when magnesium ions are added after the potassium ions instead of simultaneously? This is challenging to realize at protein concentrations suitable for SAXS because the filament solution is very viscous and controlled mixing (with a well defined assembly time of the filaments) is nearly impossible. By adapting our microfluidic devices by adding additional inlets for magnesium chloride downstream from the original intersection we can mix magnesium into (partially) pre-assembled vimentin in a well controlled manner and compare the scattering from both intermediate and equilibrium states to samples where both potassium and magnesium have been added simultaneously. 


\section{Appendix A}

\section{Purification of vimentin}

This section gives protocols for the expression and purification of vimentin in E.coli bacteria. The plasmid for the transformation was kindly provided by Harald Herrmann, DKFZ Heidelberg, Germany. These protocols have been adapted by Susanne Bauch who also prepared most of the protein. Unless indicated otherwise, all chemicals are from Carl Roth GmbH+Co. KG (Karlsruhe, Germany).

\section{A.1 Culture of E.coli}

One vial $100 \mu \mathrm{l}$ E.coli (T1 cells, \#T3017, Zymo research, Irvine, California, USA; stored at $-80{ }^{\circ} \mathrm{C}$ ) is defreezed on ice for $3 \mathrm{~min}$ and directly transfected by the addition of $1 \mu \mathrm{l}$ plasmid and gentle mixing. $100 \mu \mathrm{l}$ of the bacteria are then incubated for 5 min on ice and $50 \mu \mathrm{l}$ of the bacteria solution are spread out on a warm lysogeny broth (LB) agar plate (SIGMA L5667). The agar plate is warmed in an incubator (Ecotron, Infors HT, Bottmingen, Germany) at $37{ }^{\circ} \mathrm{C}$ over night. Three bacteria colonies are then transferred into seperate $5 \mathrm{ml}$ autoclaved terrific broth solution (47,6 g TB (SIGMA, \#T0918) and $8 \mathrm{ml}$ 99,5\% glycerol) dissolved in $900 \mathrm{ml}$ destillated $\mathrm{H}_{2} \mathrm{O}$ and filled up to $\left.1 \mathrm{l}\right)$ with $5 \mu \mathrm{l}$ ampicillin solution $(100 \mathrm{mg} / \mathrm{ml}$ ampicilin sodium salt, final concentration 1:1000, SIGMA) and grown for $6 \mathrm{~h}$ at $37^{\circ} \mathrm{C}$ and $150 \mathrm{rpm}$ in the incubator. Afterwards, each bacteria solution is transferred to $1 \mathrm{l}$ terrific broth solution (with $0.5 \mathrm{ml}$ ampicillin solution) and continues to grow at $37^{\circ} \mathrm{C}$ and $150 \mathrm{rpm}$ over night. The bacteria are then seperated from the growth medium by $12 \mathrm{~min}$ centrifugation at $4500 \mathrm{~g}$ and $4{ }^{\circ} \mathrm{C}$ (Eppendorf centrifuge $5810 \mathrm{R}$, Eppendorf, Hamburg, Germany). The bacterial pellet is stored at $-20^{\circ} \mathrm{C}$ until purification. 


\section{A.2 Extraction of vimentin from the E.coli pellet}

\section{Required buffers and solutions}

- lysis buffer: 50 mM TRIS , $25 \%$ saccharose , 1 mM EDTA di-sodium salt, pH 8.0 adjusted with $\mathrm{HCl}$

- lysozyme solution: $120 \mathrm{mg}$ lysozyme dissolved in $12 \mathrm{ml}$ lysis buffer

- detergent buffer: $200 \mathrm{mM} \mathrm{NaCl}, 1 \%$ NP40, 1\% DOC, 20 mM TRIS, 2 mM EDTA, pH 7.5 adjusted with $\mathrm{HCl}$

- GII buffer: 10 mM TRIS, 0,5 v-\% TritonX-100, 5mM EDTA, pH 8.0 adjusted with $\mathrm{HCl}$

- GII/KCl solution: $1.5 \mathrm{M} \mathrm{KCl}$ in GII buffer

- TRIS-EDTA buffer: 10 mM TRIS, 0.1 mM EDTA, pH 8.0 adjusted with HCl

- DNAse1 solution: 50 mg/ml DNAse1 (Sigma-Aldrich Corporation, St. Louis, MO, USA) in $100 \mathrm{mM}$ TRIS, $100 \mathrm{mM} \mathrm{NaCl}$, pH 7.5

- RNAseA solution: 10 mg/ml RNAseA (Roche Diagnostics, Mannheim, Germany) in 100 mM TRIS-HCl, pH 7.5

- PMSF solution: saturated PMSF (Phenylmethylsulfonylfluorid) solution in ethanol

- DP buffer $(10 \times)$ : 50 mM TRIS, 10 mM EDTA, 1 mM EGTA, pH 8.4 adjusted with $\mathrm{HCl}$

- DP buffer $(1 \times)$ : 5 mM TRIS, 1 mM EDTA, 0.1 mM EGTA, 1 mM DTT, pH 8.4 adjusted with $\mathrm{HCl}$ before addition of DTT

- column buffer: $400 \mathrm{ml} 10 \mathrm{M}$ urea, $50 \mathrm{ml}$ DP buffer $(10 \times)$, $500 \mu \mathrm{l}$ DTT, pH 7.5 adjusted

- DEAE sepharose: $30 \mathrm{ml}$ DEAE (diethylaminoethyl) sepharose stock solution (GE Healthcare, Munich, Germany) + $30 \mathrm{ml}$ column buffer

- CM sepharose:30 ml CM (carboxymethyl) sepharose stock solution (GE Healthcare, Munich, Germany) + $30 \mathrm{ml}$ column buffer 


\section{Purification}

The bacterial pellets are dissolved in a total of $48 \mathrm{ml}$ lysis buffer and separated into two dounce homogenisators $(20.05 \mathrm{~mm} \pm 0.025 \mathrm{~mm}$ pestle, VWR, Darmstadt, Germany). $6 \mathrm{ml}$ lysozyme solution is added to each homogenisator and the pellet is homogenized and incubated for 30 min on ice. Afterwards, $300 \mu \mathrm{l} 1 \mathrm{M} \mathrm{MgCl}_{2}, 33 \mu \mathrm{l}$ RNAseA solution, $30 \mu \mathrm{l}$ DNAse1 solution, $300 \mu \mathrm{l}$ PMSF solution and $600 \mu \mathrm{l} 10 \mathrm{v}-\%$ NP40 solution are added to each homogenisator, the solution is homogenised again and incubated on ice for additional $10 \mathrm{~min}$. Then $60 \mathrm{ml}$ detergent buffer, $600 \mu \mathrm{l}$ $1 \mathrm{M}$ DTT, $900 \mu \mathrm{l}$ PMSF solution and $300 \mu \mathrm{l} 50 \mathrm{mM}$ Pefabloc are added to each homogenisator, and following another homogenisation step the solution is centrifuged in six centrifuge tubes for $25 \mathrm{~min}$ at $10000 \mathrm{~g}$ and $4{ }^{\circ} \mathrm{C}$ (Beckmann centrifuge J26XP, Rotor Ja $30.50 \mathrm{Ti}$, Beckmann Coulter GmbH, Krefeld, Germany). This is followed by washing and centrifugation step 1, where the supernate is decanted, the pellets are dissolved in a total of $120 \mathrm{ml}$ GII buffer, $120 \mu \mathrm{l} 1 \mathrm{M}$ DTT, $600 \mu \mathrm{l}$ PMSF solution and $300 \mu \mathrm{l} 50 \mathrm{mM}$ Pefabloc homogenized and incubated on ice for $10 \mathrm{~min}$. The solution then is separated into four centrifuge tubes and centrifuged for 20 min at $10000 \mathrm{~g}$ and $4{ }^{\circ} \mathrm{C}$ (Beckmann centrifuge J26XP). In washing and centrifugation step 2, again, the supernate is decanted, the pellets are dissolved in a total of $120 \mathrm{ml}$ GII/KCl buffer, $120 \mu \mathrm{l} 1 \mathrm{M}$ DTT, $600 \mu \mathrm{l}$ PMSF solution and $300 \mu \mathrm{l} 50 \mathrm{mM}$ Pefabloc homogenized and incubated on ice for $10 \mathrm{~min}$. The solution then is separated into four centrifuge tubes and centrifuged for another $20 \mathrm{~min}$ at $10000 \mathrm{~g}$ and $4{ }^{\circ} \mathrm{C}$ (Beckmann centrifuge J26XP). The subsequent washing and centrifugation step 3 is identical to washing and centrifugation step 1. In washing and centrifugation step 4, the pellets are dissolved in $80 \mathrm{ml}$ TE buffer, $80 \mu \mathrm{l} 1 \mathrm{M}$ DTT, $400 \mu \mathrm{l}$ PMSF solution and $200 \mu \mathrm{l} 50 \mathrm{mM}$ Pefabloc, homogenized, incubated on ice for $15 \mathrm{~min}$ and centrifuged in two centrifuge tubes for $10 \mathrm{~min}$ at $10000 \mathrm{~g}$ and $4{ }^{\circ} \mathrm{C}$ (Beckmann centrifuge J26XP). The pellet is dissolved in $28.5 \mathrm{ml} 9.5 \mathrm{M}$ Urea, $300 \mu \mathrm{l} 1 \mathrm{M}$ TRIS-HCl $(\mathrm{pH} 7.5), 300 \mu \mathrm{l} 0.5 \mathrm{M}$ EDTA, $300 \mu \mathrm{l}$ PMSF solution and $300 \mu \mathrm{l} 1 \mathrm{M}$ DTT and homogenized. The solution then is centrifuged for $1 \mathrm{~h}$ at $31170 \mathrm{rpm}(\approx 100000 \mathrm{~g})$ at $20^{\circ} \mathrm{C}$ (Beckmann-UZ L90K, Ti70 rotor, Beckmann Coulter GmbH, Krefeld, Germany). The supernate is kept, $10 \mu \mathrm{l} 1 \mathrm{M}$ MAC solution are added per millilitre supernate and it is stored over night at $-20^{\circ} \mathrm{C}$ and then gently thawed before the ion exchange chromatography. 


\section{Ion exchange chromatography}

\section{Part 1 - anion exchanger}

The ion gradient mixer $(2 \times 50 \mathrm{ml}$, Schütt 24 , Göttingen, Germany) is filled with $40 \mathrm{ml}$ column buffer in the front chamber and $40 \mathrm{ml}$ column buffer with $0.448 \mathrm{~g} \mathrm{KCl}$ added. The DEAE sepharose is filled into the chromatography column (Econo chromatography column, $25 \mathrm{~mm}$ diameter, $200 \mathrm{~mm}$ long, Biorad, Munich, Germany), allowed to settle and carefully washed with $40 \mathrm{ml}$ column buffer. Then the protein solution is added and the conditions of the column are adjusted such that there is a slow flow with a constant filling level of the column. The outflow from the column is collected. The column is once again washed with $40 \mathrm{ml}$ column buffer (slowly and at a constant level). When the collected total outflow volume of the column reaches the dead volume of the column the actual sample collection begins. After the washing, the gradient mixing is started and the gradient solution flows into the column at about 3 to 4 drops per second. The flow into and out of the column are then adjusted such that the volume in the column remains constant and the outflow is in the range of 7 to 9 drops per second. The outflow is collected in partitions of about $1.5 \mathrm{ml}$ and each partition is monitored for its protein content (see sec. 2.3). The partitions containing protein are kept and combined for the cation exchanger column.

\section{Part 2 - cation exchanger}

The cation exchange column chromatography is analogous to the anion exchange column chromatography except that the column material is CM sepharose instead of DEAE sepharose and that the salt concentration in the gradient mixer is twice as high. After the cation exchanger $10 \mu \mathrm{l} 1 \mathrm{M}$ MAC solution are added per millilitre sample and the sample is stored at $-80^{\circ} \mathrm{C}$.

\section{Quality control}

The purity of the extracted vimentin is controlled by an SDS (sodium dodecyl sulfate) PAGE gel. 


\section{Buffers}

- electrophoresis buffer: 0.4 M Tricine, 0.6 M TRIS, 1 \% SDS, 25 mM Nahydrogensulfit at $\mathrm{pH}$ 8.2-8.3

- sample buffer: $150 \mu \mathrm{l} 4 \times$ sample buffer for gels (VWR/CBS, ordering no. FB31010), $270 \mu \mathrm{l} \mathrm{H}{ }_{2} \mathrm{O}, 60 \mu \mathrm{l}$ DTT $10 \times$ (VWR/CBS, ordering no. 32001)

\section{Gel run}

A CBS gel (TEO) with $8 \%$ acrylamide (VWR/CBS, ordering no. FK00812-10) is mounted in DCX 700 gel chamber (VWR). $10 \mu \mathrm{l}$ each of sample and controls of interest are mixed with $40 \mu \mathrm{l}$ sample buffer and denaturated for $10 \mathrm{~min}$ at $70{ }^{\circ} \mathrm{C}$. Afterwards the solutions are quickly centrifuged (Eppendorf MiniSpin, Eppendorf, Hamburg, Germany) and $10 \mu \mathrm{l}$ from the top are placed in one lane of the gel. On at least one of the lanes, a gel marker ("Protein Marker", pre-stained 6.5-200, AppliChem, Darmstadt, Germany) is run. The electrophoresis is performed at a maximum voltage of $200 \mathrm{~V}$ and a constant current of about $100 \mathrm{~mA}$ until the bands are well seperated (roughly $1.5 \mathrm{~h}$ ). The finished gel is stained with InstantBlue ${ }^{T M}$ (Expedeon, San Diego, California, USA) for 15 to 60 minutes and subsequenlty rinsed with water to remove excess dye. A single, isolated band at $53 \mathrm{kDa}$ indicates purified vimentin. 


\section{Appendix B}

\section{Derivation of form factors for SAXS}

This appendix presents some details for the derivation of SAXS form factors which were previously skipped.

\section{B.1 Seperation of longitudinal and cross-sectional components}

In section 3.2 we stated that the form factor for long, rod-like particles can be separated into longitudinal and cross-setional components as follows:

$$
I(q)=F_{L} \cdot F_{c}
$$

where

$$
F_{L}(q)=\int_{0}^{\infty}\left(\frac{\sin (q L \gamma / 2)}{(q L \gamma / 2)}\right)^{2} \mathrm{~d} \gamma=2 \frac{\operatorname{Si}(q L)}{q L}-\left(\frac{\sin (q L / 2)}{q L / 2}\right)^{2}
$$

is the form factor for an infinitely thin rod [72] and

$$
F_{c}=\left|\int_{A} \rho\left(\vec{r}_{c}\right) e^{i \vec{q} \vec{r}_{c}} \mathrm{~d}^{2} r_{c}\right|^{2}
$$

Here, we show that this is, indeed, valid, following the argument given by Porod in $[69]$.

First, we define a cylindric system such, that the long axis of the rod lies along the z-axis. Hence, $\rho(\vec{r})=\rho_{c}\left(\vec{r}_{c}\right) \Theta(z-L / 2) \Theta(z+L / 2)$. We define $\alpha$ as the angle between the scattering vector and the z-axis and $\theta$ as the angle of $\vec{q}$ in the cross-sectional 
plane.

Equation 3.4 then becomes

$$
I(\vec{q})=\iint \mathrm{d} \alpha \mathrm{d} \theta\left|\int_{V} \rho_{c}\left(\vec{r}_{c}\right) \Theta(z-L / 2) \Theta(z+L / 2) e^{i \vec{q} \vec{r}_{c}} e^{i q z \cos (\alpha)} \mathrm{d}^{2} r_{c} \mathrm{~d} z\right|^{2}
$$

The integration over $z$ can be performed separately and we obtain

$$
I(\vec{q})=\int_{0}^{\pi} \mathrm{d} \alpha\left(L \frac{\sin (q L \cos \alpha / 2)}{q L \cos \alpha / 2}\right)^{2}\left|\int_{A} \rho_{c}\left(\vec{r}_{c}\right) e^{i \vec{q} \vec{r}_{c}} \mathrm{~d}^{2} r_{c}\right|^{2}
$$

For $q L \gg 1$ the term $\left(L \frac{\sin (q L \cos \alpha / 2)}{q L \cos \alpha / 2}\right)^{2}$ rapidly decays as $\alpha$ increases. Therefore, only rods with the longitudinal axis nearly perpendicular to the scattering vector contribute to the signal. As $\vec{q} \vec{r}_{c}=q r_{c} \cos \theta \sin \alpha$ remains nearly unchanged by small changes in $\alpha$ near $\pi$, we can approximate equation B.5 to equation B.1.

\section{B.2 The effect of longitudinal polydispersity on the form factor}

From equation 3.7 and equation 3.10 it is obvious that for very long filaments the scattering from a longitudinally polydisperse ensemble of filaments is equal to the scattering of filaments of the mean length as the form factor is linear in the filament length. But even for filaments which are not sufficiently long to be described by equation 3.10 for a monodisperse system, this approximation can hold for polydisperse systems as equation 3.8 approaches equation 3.10 oscillatingly. Therefore, for Gaussian distributions, the deviations from equation 3.10 mostly cancel out. 


\section{B.3 Form factor of a very long cylinder}

For a very long cylinder with radius $R$ and length $L$ the form factor is given by (see e.g. $[69,79])$

$$
\begin{aligned}
I(q)= & L^{2} P_{L} \cdot F_{c} \propto \frac{L^{2}}{q L}\left|\int_{0}^{\pi} \int_{0}^{R} \mathrm{~d} \theta 2 \pi r d r e^{i q r \cos \theta}\right|^{2} \\
& \propto \frac{L^{2}}{q L}\left|\int_{0}^{R} 2 r \mathrm{~d} r B_{0}(q r)\right|^{2} \propto \frac{L^{2}}{q L}\left(\frac{R^{2} B_{1}(q R)}{q R}\right)^{2}
\end{aligned}
$$

where $B_{0}$ and $B_{1}$ are the zeroth and first order Bessel function of the first kind. The integral over $\theta$ follows from the identity

$$
B_{n}(x)=\frac{-i^{n}}{\pi} \int_{0}^{\pi} e^{i x \cos \phi} \cos n \phi d \phi
$$

applied to $n=0$ and the integral over $r$ follows from

$$
\frac{\mathrm{d} B_{n}(x)}{\mathrm{d} x}=-\frac{n}{x} B_{n}(x)-B_{n-1}(x)
$$

applied to $x B_{1}(x)[160]$.

\section{B.4 Estimating the SAXS signal for octamers and hexadecamers}

In order to estimate the SAXS signal of vimentin during the assembly process we need to estimate the individiual signal from all contributing species. We estimate the signal of octamers $I_{o}$ and hexadecamers $I_{h}$ by asuming that they correspond to two parallel tetramers at a distance $d$ respectively to four parallel tetramers in a square with side length $d$. This then corresponds to a local structure factor and we can estimate

$$
I_{o / h}(q)=\left\langle N^{2} S_{o / h}(\vec{q}) F_{t e t}(\vec{q})\right\rangle
$$

where $N$ is the number of tetramers, i.e. either 2 or $4, S_{o / h}(q)$ is the structure factor corresponding to the octamer respectively hexadecamer geometry and $F_{\text {tet }}(q)$ the form factor of a vimentin tetramer. When we assume that each tetramer has 
a centrosymmetric cross-section we can perform the radial averaging of each term separately and obtain according to [67]

$$
\left\langle F_{\text {tet }}(\vec{q})\right\rangle=F_{\text {tet }}(\vec{q})
$$

where $F_{t e t}(\vec{q})$ corresponds to the scattering of tetrameric vimentin,

$$
\left\langle S_{o}(\vec{q})>=1+\frac{1}{2}\left\langle 2 e^{i q d \cos \theta}\right\rangle=1+\frac{1}{2} 2 B_{0}(q d)\right.
$$

and

$$
\begin{aligned}
\left\langle S_{h}(\vec{q})\right\rangle & =1+\frac{1}{4}\left\langle 8 e^{i q d \cos \theta}+4 e^{i q \frac{\sqrt{2}}{2} d \cos \theta}\right\rangle \\
& =1+\frac{1}{4}\left(8 B_{0}(q d)+4 B_{0}\left(q \frac{\sqrt{2}}{2} d\right)\right)
\end{aligned}
$$




\section{Appendix C}

\section{MatLab code for calculating SAXS form factors}

In 4.4.2, we introduced eq. 4.9 and 4.10 as SAXS form factors for vimentin filaments. In this appendix we provide the implementation of the form factor in MatLab we used in the fitting procedure.

function $F=$ objfunVec $(x c)$

\%---Cost function to minimize-----

\% form factor cylinder with attached Gaussian chains

$\%$ includes polydispersity and tetrameric component

$\%$ put together all parameters and data needed

global DATA;

global TET;

$\%$ select $q$-range

jlow $=1$;

jup $=1$;

for $i=1: \operatorname{size}(\operatorname{DATA}(:, 1))$

if $\operatorname{DATA}(i, 1)<=0.07 \%$ lower limit 


$$
\text { jlow }=i \text {; }
$$

end

if $\operatorname{DATA}(i, 1)<=3 \%$ upper limit

$$
\text { jup }=i
$$

end

end

$y=\operatorname{DATA}($ jlow: jup, : ) ;

$\%$ scattering vector

$\mathrm{q}=\mathrm{y}(:, 1)$;

$\%$ tetramer data

tetdat $=\operatorname{TET}($ jlow: jup, 2$)$;

$\%$ setup parameters

$\%$ radius $R$ in $n m$

$\mathrm{R}=\mathrm{xc}(1)$;

$\%$ gyration radius $R g$ in $n m$

$\mathrm{Rg}=\mathrm{xc}(2) ;$

$\%$ shell distance in terms of $R g$

$\mathrm{dr}=0$;

\% "chain density" lambda

lambda $=1.34$;

$\%$ scaling factor gamma

gamma $=\mathrm{xc}(3)$;

$\%$ scaling ration beta

$\mathrm{bc} 2 \mathrm{bs}=\mathrm{xc}(4)$;

$\%$ constant background $c$

$c=0$

$\%$ tetramer

$\mathrm{t}=\mathrm{xc}(5)$;

$\%$ relative radial polydispersity 
sigma $=0.134$

$\%$ calculate scaling factors

a1 = gamma;

a2 =gamma $* 1$ ambda $*(\text { bc } 2 b s)^{\wedge} 2$;

$\mathrm{a} 3=2 *$ gamma $* \mathrm{bc} 2 \mathrm{bs}$;

$\mathrm{a} 4=\operatorname{gamma} *(\mathrm{bc} 2 \mathrm{bs})^{\wedge} 2$;

$\%$ model radial polydispersity

$r s=\operatorname{sigma} * \mathrm{R}$;

$R s=\max (0.01,(R-3 * r s)):(r s / 100):(R+3 * r s) ; \%$ array of radii

$\mathrm{dR}=\operatorname{ones}(1$, length $(\mathrm{Rs})) * \mathrm{Rg} * \mathrm{dr} ; \%$ array of chain-core distances

$\%$ weight of radii

Rdist $=(1 /(100 * \operatorname{sqrt}(\operatorname{pi} * 2))) * \exp \left(-(\operatorname{Rs}-\mathrm{R}) \cdot{ }^{\wedge} 2 /\left(2 * r \mathrm{~s}^{\wedge} 2\right)\right)$;

\% do actual form factor calculation

$\%$ cylindrical cross-correlation

$f C s=2 * \operatorname{besselj}(1, q * R s) . /(q * R s)$;

$\mathrm{FCs}=\left(\mathrm{fcs} .{ }^{\wedge}(2)\right) *$ Rdist' $;$

$\mathrm{Fl}=\mathrm{pi} \cdot / \mathrm{q}$;

$\mathrm{FS}=\mathrm{FCS} \cdot * \mathrm{Fl}$;

$\%$ gaussian cross-correlation

$\mathrm{x}=(\mathrm{q} * \mathrm{Rg}) \cdot{ }^{\wedge} 2$;

$\mathrm{FC}=\left(2 *(\exp (-\mathrm{x})-1+\mathrm{x}) \cdot /\left(\mathrm{x} \cdot{ }^{\wedge} 2\right)\right)$;

\% core-chain correlation

psi $=(1-\exp (-\mathrm{x})) \cdot / \mathrm{x}$;

$\mathrm{Ssc}=\operatorname{psi} *(f \mathrm{fs} . * \operatorname{besselj}(0, \mathrm{q} *(\mathrm{Rs}+\mathrm{dR})) *$ Rdist' $) . * \mathrm{Fl}$;

\% chain-chain correlation

$\mathrm{Scc}=\operatorname{psi} *\left(\left(\left((\operatorname{besselj}(0, \mathrm{q} *(\mathrm{Rs}+\mathrm{dR}))) .{ }^{-}(2)\right)\right) *\right.$ Rdist $\left.{ }^{\prime}\right) . * \mathrm{Fl}$; 
\% put together entire model function $g$

$\mathrm{g}=(1-\mathrm{t}) *(\mathrm{a} 1 * \mathrm{Fs}+\mathrm{a} 2 * \mathrm{Fc}+\mathrm{a} 3 * \mathrm{Ssc}+\mathrm{a} 4 * \mathrm{Scc})+\mathrm{t} *$ tetdat $+\mathrm{c} ;$

\% calculate cost function \% define vector valued function,

$\%$ needed as input for lsqnonlin(...)

$\mathrm{f}=\mathrm{y}(:, 3)$;

$F=((g-y(:, 2)) . / f)$; 


\section{Appendix D}

\section{Code for evaluating COMSOL $4.0 \mathrm{a}$ simulations}

In chapter 6 we discussed which data we extract from the COMSOL 4.0s simulations and the basic principles of the postprocessing. This appendix gives the MATLAB code used for evaluation, including some comments on the reasons for this implementation. Some commands are specific to the computer dancer at the Institute for X-Ray Physics in Göttingen and are marked as such.

\section{D.1 Calculating streamlines}

The first step of evaluating data along the streamlines is the calculation of the streamlines. This could in principle be done directly in COMSOL 4.0a for stream lines starting along one cut line in the geometry, but for lanes starting in one cut plane in the geometry, as we require them, accessing COMSOL via MatLab allows for the necessary automatization.

\% change to path of relevant simulation and create directory for streamline data cd SLS09/200mMKC1_80_80_40

mkdir lines

$\%$ start connection to COMSOL4.Oa

addpath /opt/comsol/COMSOL40a/mli \% Path of the MatLab LiveLink 
of the COMSOL installation, this depends on the system used !comsol40a server > out.dat \& \% This gives a command to the system to start the COMSOL server

and to return all output of the server to out.dat

import com.comsol.model.*

import com.comsol.model.util.* \% Import relevant Java classes to MatLab

mphstart \% Starts the COMSOL connection in MatLab model $=\operatorname{mphload}($ 'out.mph') $\%$ Load the solved simulation

\% Actual stream lines calculation

model.result.create('pg11',3) \% Create a plot group in COMSOL $\%$ Add stream lines to the plot group streams $=$ model.result ('pg11'). feature.create('streams', 'Streamline') startx $=100 ; \% x$-coordinate of start point for all stream lines starty $=[350: 1: 500] ; \% y$-coordinates of start points, this corresponds to a 1 um spacing

$\%$ Set parameters for stream line calculation: Use fixed points as start points and extrafine resolution streams.set ('startmethod', ' coord'); streams.set ('xcoord', startx); streams.set ('ycoord', starty); streams.set ('resolution', 'extrafine');

\% Loop the z-position and calculate streamlines starting at $x$ and $y$ positions defined above for all $z$, here a $1 \mu \mathrm{m}$ spacing in the central (shallower) inlet for startz $=66: 120$

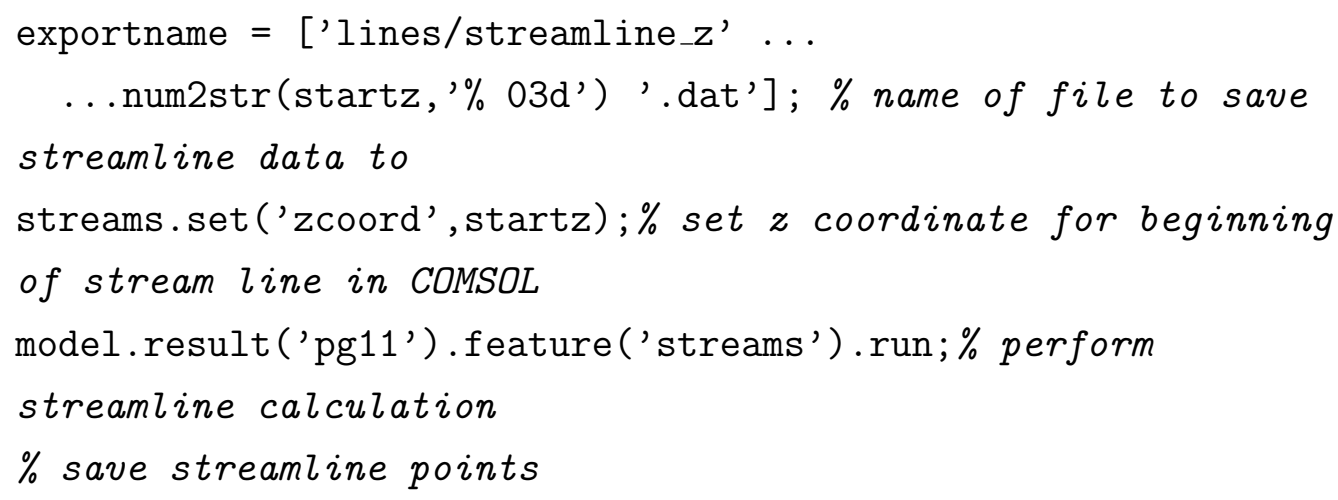




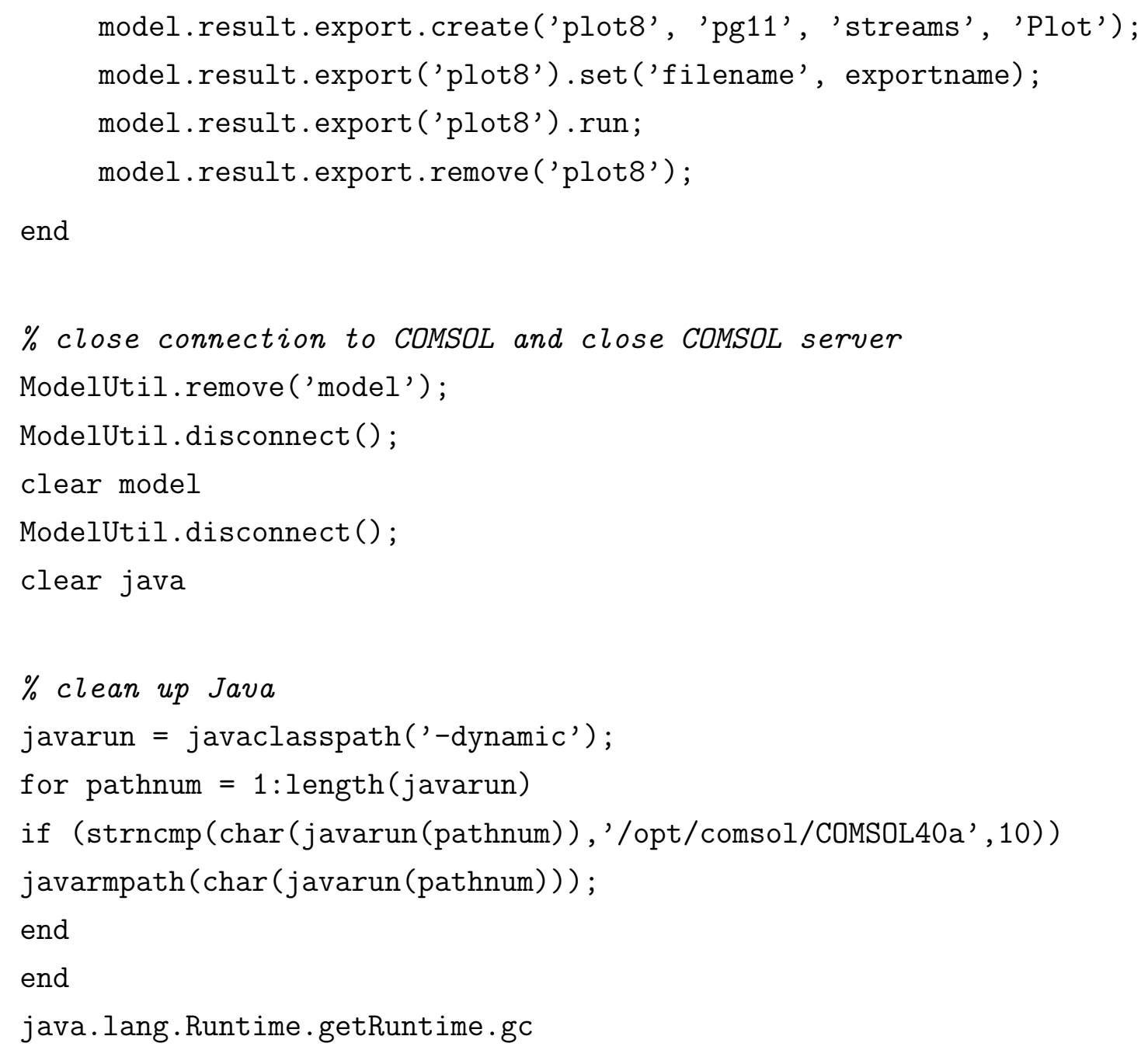

\section{D.2 Evaluating the simulation along the stream- line}

Now that we know the coordinates of each streamline, we need to extract the flow field and solute concentrations at each of these points from the simulation. The MatLab LiveLink for COMSOL provides a function to do so. It is, however, limited in the maximal number of points that can be evaluated at once due to memory issues. Therefore, most of the following code attempts to regularly free memory for both COMSOL (by regularly restarting it) and MatLab.

\% change to path of relevant simulation

cd SLS09/200mMKCl_80_80_40 
$\%$ loop over selected $z$ (for which streamlines exist)

for $z=90: 106$

\% open and connect COMSOL, load simulation

addpath /opt/comsol/COMSOL40a/mli

! comsol40a server > out.dat \&

import com.comsol.model.*

import com.comsol.model.util.*

mphstart

model $=\operatorname{mphload}($ 'out $\cdot \mathrm{mph}$ ')

\% disable tracking of model history in COMSOL,

this saves memory

model.hist().disable()

\% load stream line coordinates

lines_name $=[$ 'linesstreamline_z' num2str (z, '\% 03d') '.dat'];

fid $=$ fopen(lines_name); \% open file

if $(\mathrm{fid}==-1)$

$\%$ catch non-valid files

disp(sprintf('Could not find file \% s.',lines_name))

return \% leave macro

else

for header $=1: 8 \%$ skip file header

line $1=$ fgetl $(f i d)$

end

$\%$ read data into array

lines $=f \operatorname{scanf}\left(f i d,{ }^{\prime} \%\right.$ e $\%$ e $\backslash n^{\prime},[4$, inf $\left.]\right) ;$

lines $=$ lines'; \% transpose array

fclose(fid); \% close file

end

$\%$ evaluate array in groups of 10000 points

for step $=1$ : steps

if $\bmod (\mathrm{step}, 20)==0 \%$ clean up memory after steps

$\%$ disconnect from COMSOL, save relevant data

ModelUtil.remove ('model')

ModelUtil.disconnect() 
clear model

save ('interim.mat', 'z', 'step', 'steps', 'lines');

ModelUtil.disconnect()

clear java

\% free Java memory

java.lang. Runtime.getRuntime.gc

javarun = javaclasspath ('-dynamic');

for pathnum $=1:$ length $($ javarun $)$

if $(\operatorname{strncmp}(\operatorname{char}(j \operatorname{avarun}($ pathnum $)), \ldots$

...'/opt/comsol/COMSOL40a', 10))

javarmpath (char (javarun (pathnum)));

end

end

clear java

java.lang. Runtime.getRuntime.gc

$\%$ restart connection to COMSOL

import com.comsol.model.*

import com.comsol.model.util.*

load/home/mbrenni/Documents/interim.mat

serv $=$ ['! comsol40a server > outtestz' $\ldots$

...num2str (step, '\% 03d') '.txt \&'];

eval(serv); \% this executes the command

defined in the string above

mphstart

model $=\operatorname{mphload}($ 'out $\cdot \mathrm{mph}$ ')

model.hist().disable()

end

\% initialize array for simulation data

dat $=\operatorname{zeros}(10000,6)$;

$\%$ set coordinates of evaluation

evalcoord $=[$ lines $((($ step -1$) * 10000)+1$ : step*10000,1) $\ldots$

.. $\operatorname{lines}((($ step -1$) * 10000)+1$ : step*10000,2) ...

...lines $(($ step -1$) * 10000)+1$ : step*10000,3) ]' ;

\% Evaluate the simulation at given coordinates

$\%$ magnitude of the velocity field

$\operatorname{dat}(:, 1)=\operatorname{mphinterp}(\operatorname{model}$, 'spf.U', 'coord', evalcoord)'; 


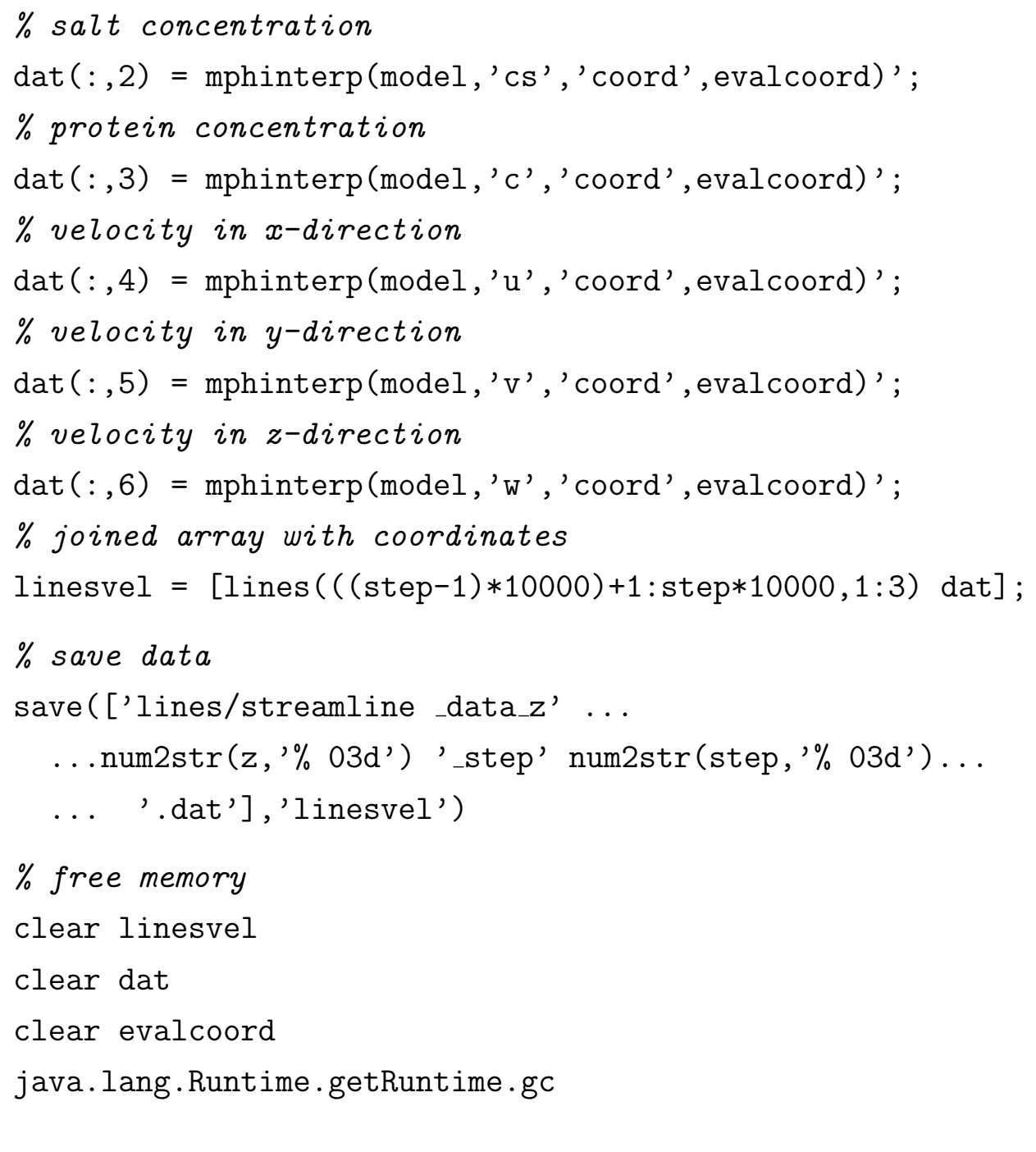

end

\% evaluate remaining data points

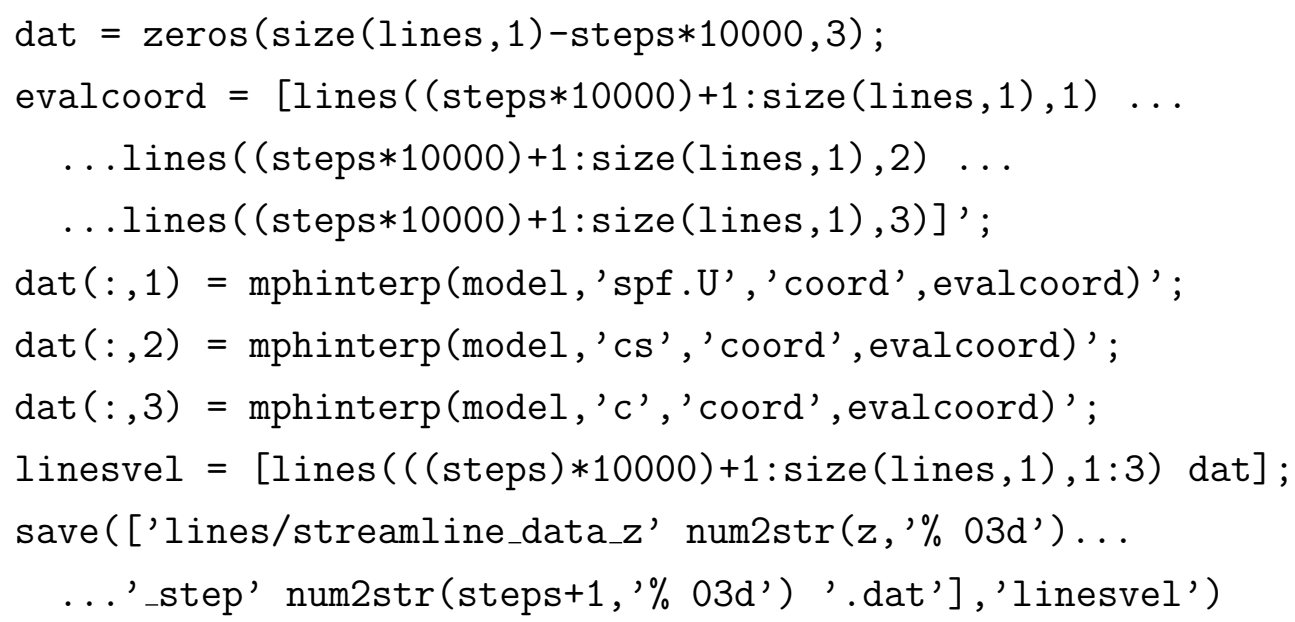

\% clear memory and close COMSOL

clear linesvel 


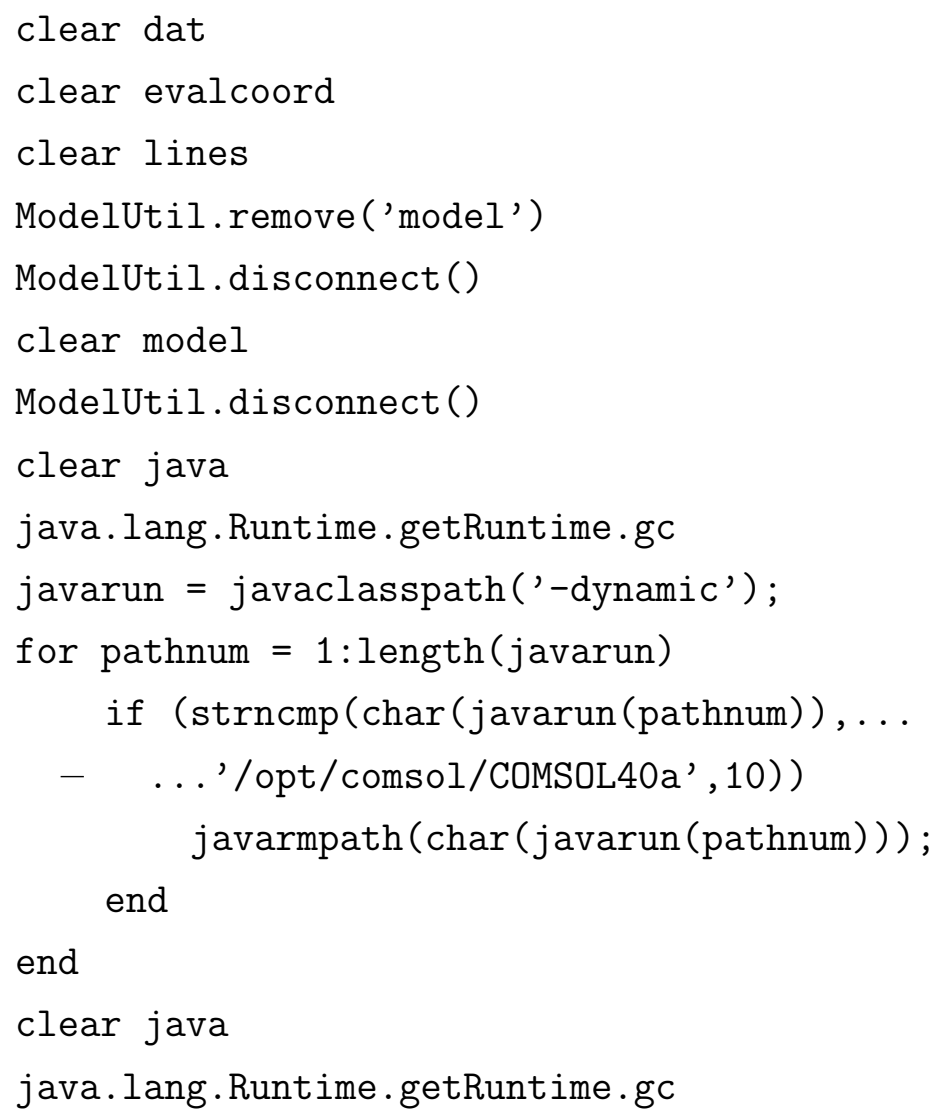

\section{D.3 Integration along the streamlines}

Following the extraction of the simulation results from COMSOL, we need to obtain the assembly times of vimentin along the streamlines. This is achieved by integrating over the inverse velocity starting from a minimum salt concentration. For easier coding, the streamline data from the preceding evaluation step are joined in a three dimensional array where each index in the third dimension corresponds to a single streamline.

\% change to path of relevant simulation, add path of for relevant functions

cd SLS09/200mMKCl_80_80_40

addpath MATLAB/comsollink

zpos $=66: 120 ; \% z$ coordinates of start points 
\% Load data of one streamline to estimate required size of arrays $\mathrm{z}=100 ; \% z$ coordinate of starting point

$\mathrm{s}=1$;

$\mathrm{d}=$ loadstream $(\mathrm{z}) ; \%$ Load all data from streamline evaluation for this $z$ coordinate of the starting point into array $d$ firstlength $=0 ; \%$ number of points in a streamline with $x$ coordinate smaller than the starting coordinate secondlength $=0 ; \%$ number of points in a streamline with $x$ coordinate larger than the starting coordinate counter $=1$; \% position in streamline array $\%$ each streamline begins with points towards smaller $x$ from the start while (d (counter, 1$)>d($ counter $+1,1))$

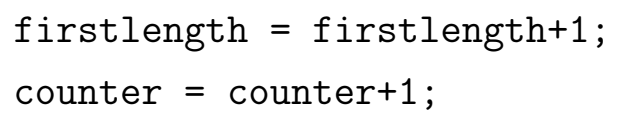

end

$\%$ afterwards the $x$ coodinate increases along the streamline while $(\mathrm{d}$ (counter, 1$)<\mathrm{d}($ counter $+1,1))$

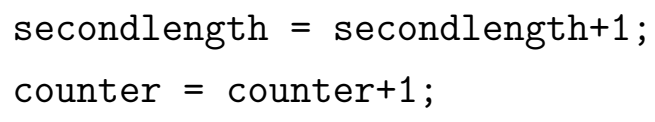

end

$\%$ create array

length $=$ secondlength + firstlength; $\%$ total length of a stream line $\%$ total number of lines:

lines for one $z$ value times number of $z$ values evaluated numberoflines $=$ idivide $(\operatorname{int32}(\operatorname{size}(d, 1)), \ldots$

...int32((length)), 'ceil')*size (zpos, 2)

$\%$ the actual arrays with -1 as default value

streamlines $=-1 *$ ones ( secondlength $+1000,9$, numberoflines +5$)$;

$\%$ start sorting

lineno $=0 ; \%$ counter for total number of lines

for $z=z p o s$ 


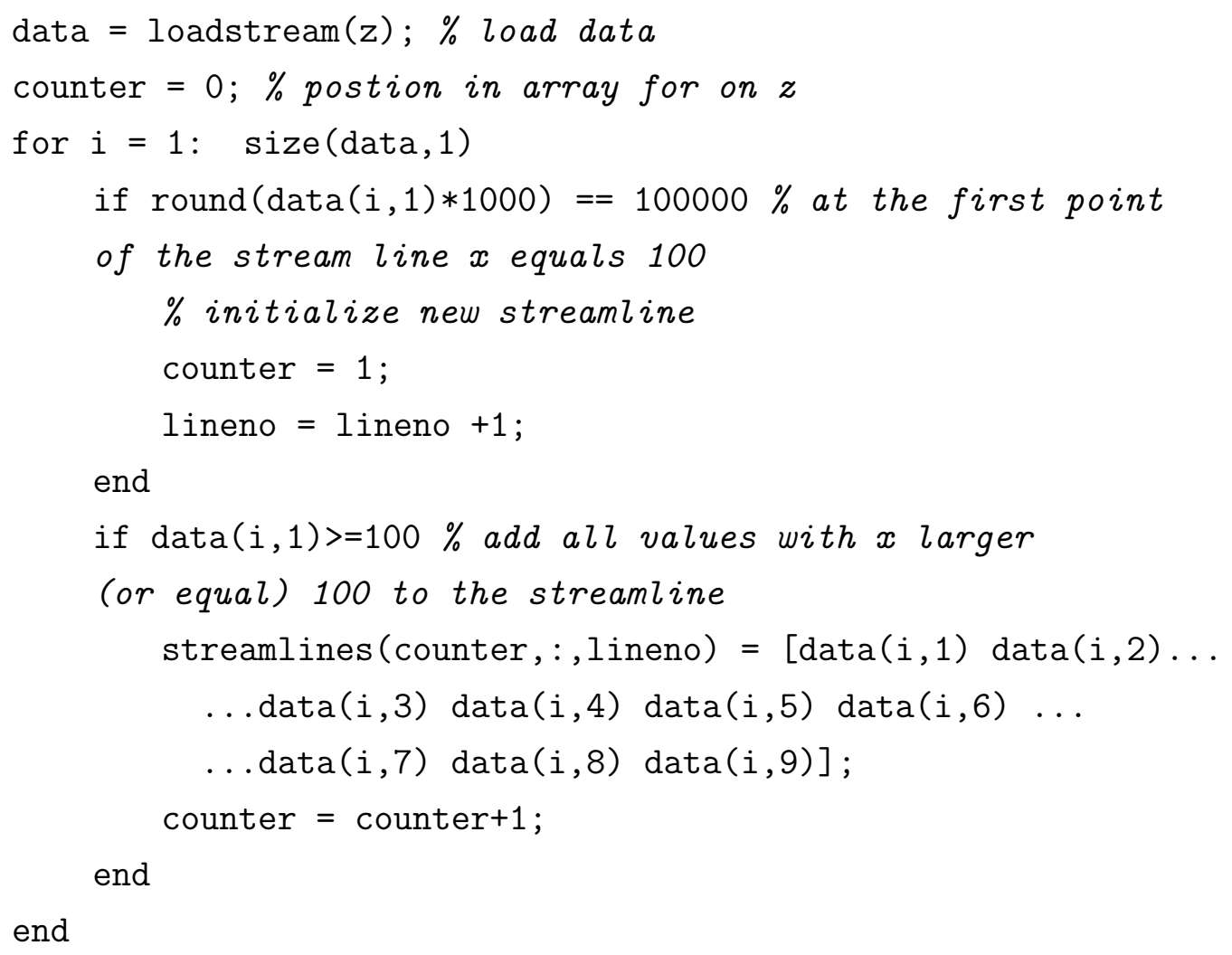

end

$\%$ set-up array to include integrated time extracteddata $=-1$ ones $(\operatorname{size}($ lines, 1$), \operatorname{size}($ lines, 2$)+1, \operatorname{size}($ lines, 3$))$; $\%$ save avaible data in array extracteddata $(:, 1: \operatorname{size}($ lines, 2$),:$ ) = lines;

\% integrate assembly time for each streamline for lineno $=1:$ size (streamlineslines, 3$) \%$ loop over streamlines tracktime $=0 ; \%$ default for integration is zero - no salt present if not((streamlineslines $(1,1,1$ ineno $)==-1)) \%$ continue only for non-default values extracteddata $(1,10,1$ ineno $)=0 ; \%$ the inital assembly time is 0 for $i=2: \operatorname{size}($ lines, 1$) \%$ follow the streamline if not((lines (i,1,lineno)==-1)) \% non default data point?

if (extracteddata $(i, 5,1$ ineno $))>=1 \%$ salt concentration higher than $1 \mathrm{mM}$ ? 


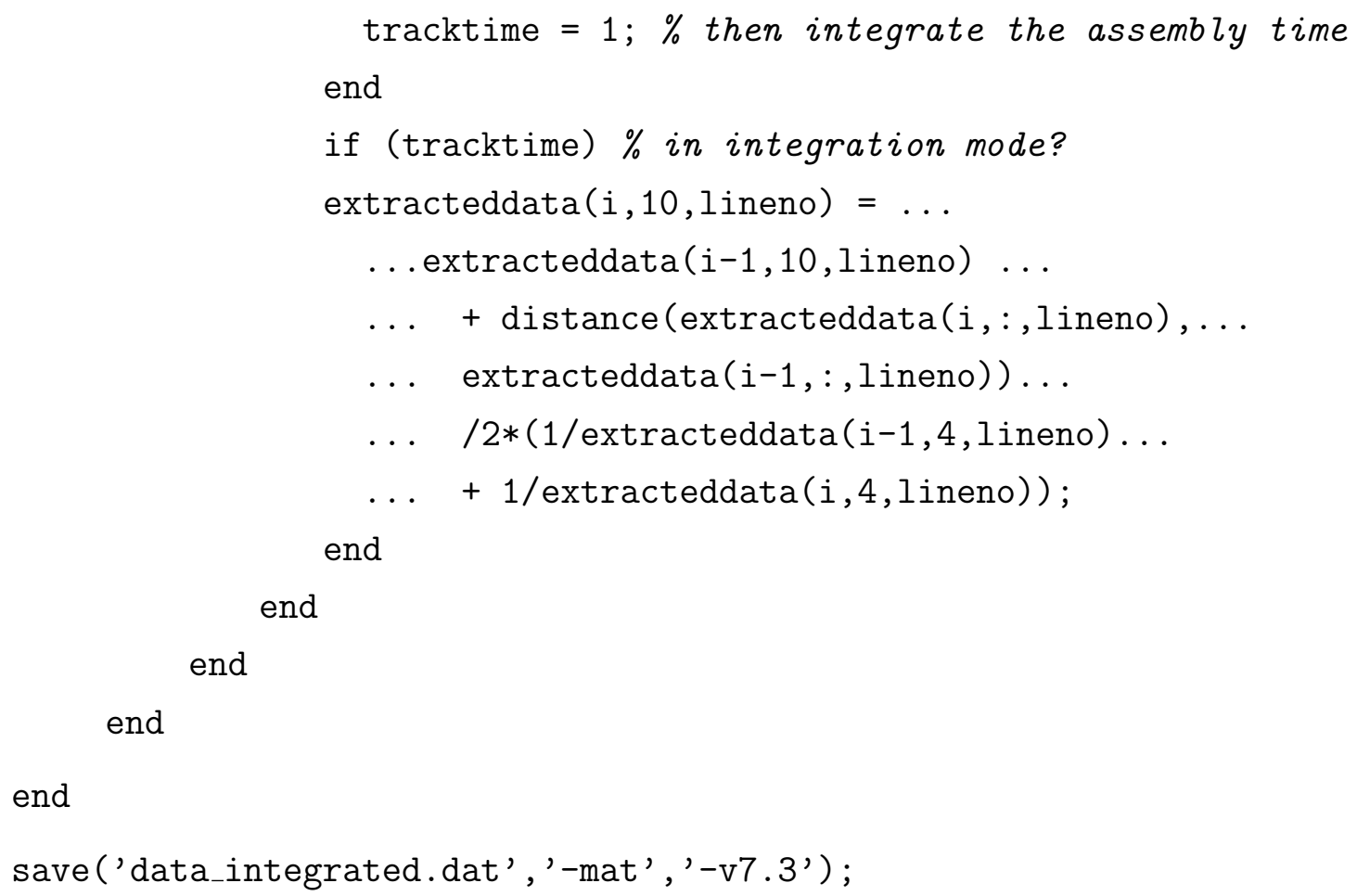

\section{D.4 Determination of ensembles}

Now that all necessary data are assembled, we can determine the ensemble of conditions (salt concentrations, assembly times...) that the x-ray probes at a certain location. For this we create arrays for each point of interest and resort the points along the streamlines into these arrays.

\% change to path of relevant simulation, and load data cd /SLS09/200mMKC1_80_80_40/

data $=$ load ('data_integrated.dat', '-mat') ;

values = data.extracteddata;

$\%$ set locations of measurement and spot size (in um)

$\mathrm{x}=200: 100: 1500$;

$\mathrm{y}=450: 50: 500$;

$\operatorname{deltax}=10$;

deltay $=2.5$;

$\%$ create data array

pointdata $=-1 *$ ones $(\operatorname{size}(x, 2), \operatorname{size}(y, 2), \ldots$ 
$\ldots \operatorname{deltax} * \operatorname{deltay} * 2 * 3 * \operatorname{size}($ values, 3$), 4)$;

$\%$ array for number of data points added to each measurement point counter $=\operatorname{ones}(\operatorname{size}(\mathrm{x}, 2), \operatorname{size}(\mathrm{y}, 2))$;

for lineno $=1:$ size (values, 3$) \%$ loop over all lines

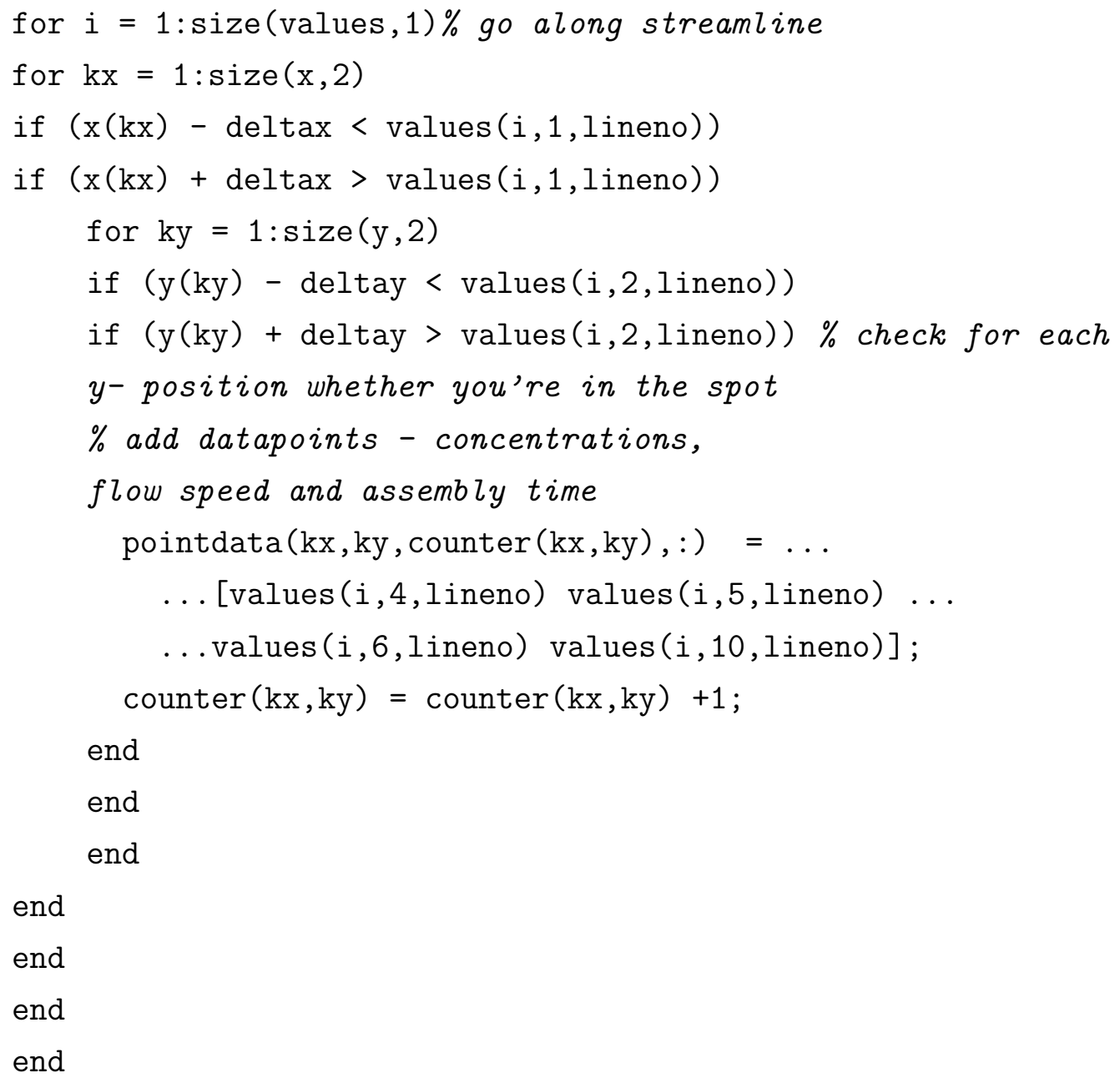

end

$\%$ save results

for $\mathrm{kx}=1: \operatorname{size}(\mathrm{x}, 2)$

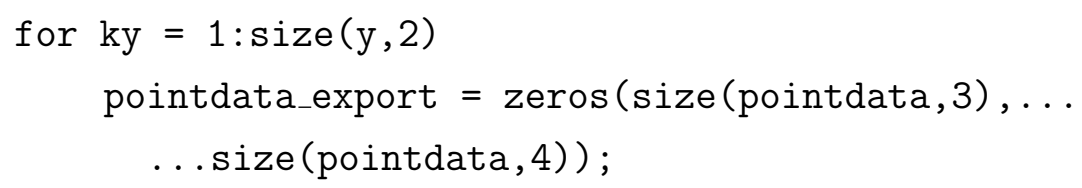




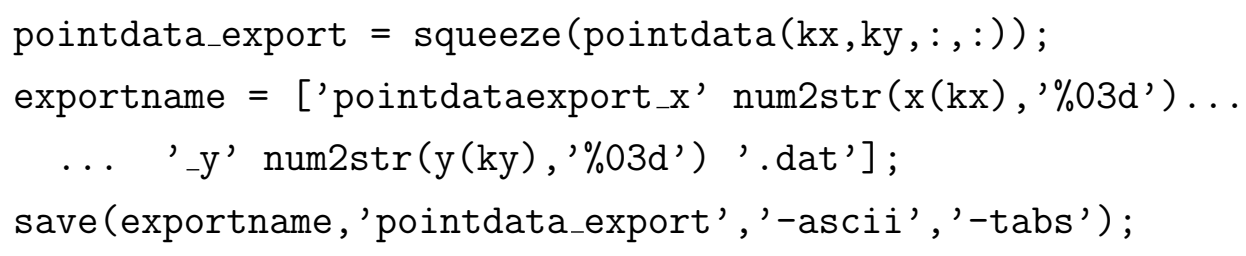

end 


\section{Appendix E}

\section{Principal component analysis}

This appendix shows and discusses the results from the principal component analysis of the SAXS signal recorded in flow in further detail. The primary components and their scores are calculated in Matlab with the princomp function.

In figures E.1a - E.1c we see the first three components obtained for the three different flow speeds. Both the form of the higher components and their scores indicate that the main information on the curve shapes is provided by these three components. We see that the general features of the components for all flow speeds are similar but that there are some distinct differences in the first component and consequently in the second component between the highest flow speed and the lower two. The first component describes the overall shape of a scattering curve and is positive for all $q$. The second component, i.e. the first correction, has both negative and positive elements and the sign is flipped for the middle flow speed. It is zero at about $0.065 \mathrm{~nm}^{-1}$. Above that, it is flatter than the first component and below that it is rather steep. Adding this component to the first component results in a flattening in the curve and a leveling off of the curve towards smaller $q$, i.e. the curve can resemble the tetrameric scattering curve. The third component has zeros at about $0.6 \mathrm{~nm}^{-1}$ and $1.1 \mathrm{~nm}^{-1}$. Except for very small $q$, its sign is inverse to the second component and it displays a very strong curvature in the double-logarithmic plot. Hence, it can be used to to describe the concave-to-convex transition.

Figure E.1d shows the sum of the first three components scaled by their score. We see that while this linear composition does not reproduce the original data, it does reproduce the main differences between the curves: the change in the overall slope, in the curvature in the mid- $q$ range and in the steepness towards low $q$. It is important to note that the reproduction of the original data does not improve by the addition 
(a) 1. Component

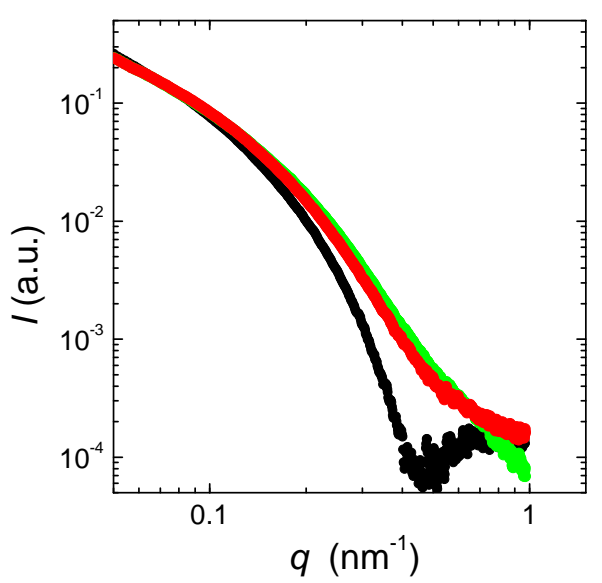

(c) 3. Component

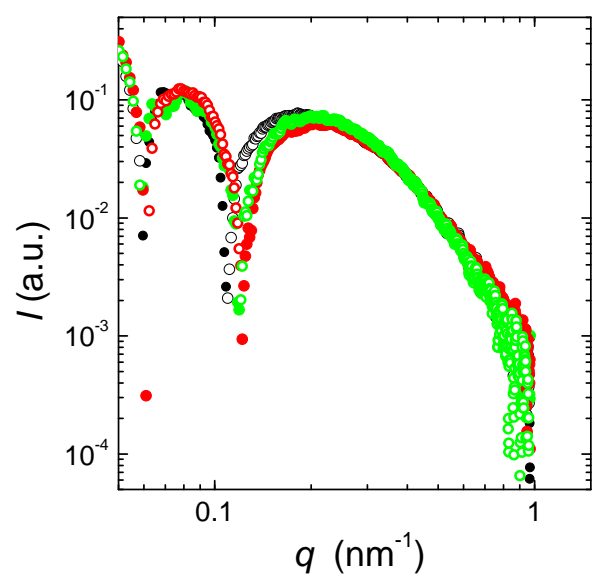

(b) 2. Component

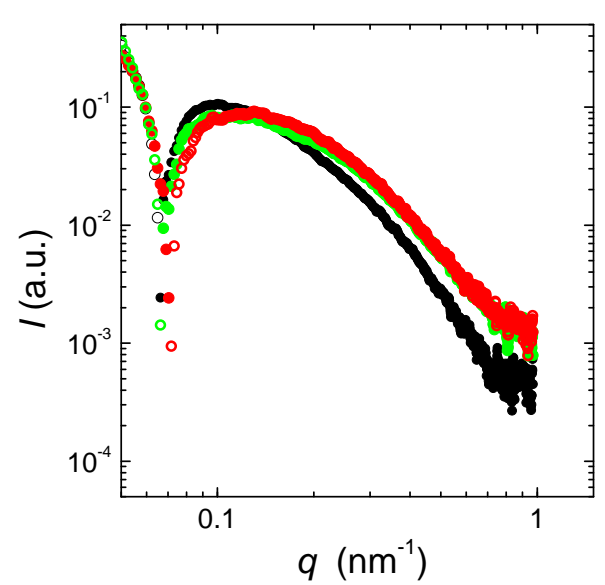

(d) Exemplary linear compositions

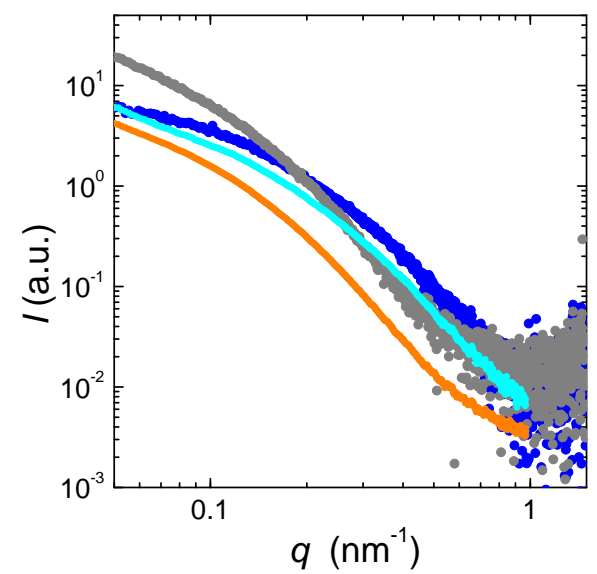

Figure E.1: (a) - (c) The first three components of the primary component analysis for flow conditions as described in figure 7.1 (black), at flow rates reduced by a factor of 2 (red) and at flow rates reduced by a factor of 4 (green). The closed symbols represent the component and the open symbol the negative part of the component. (d) Sum of the first three components scaled with their respective scores at the middle of the intersection (light blue) and $2 \mathrm{~cm}$ downstream (orange) in comparison to the original data (dark blue and gray). 
of higher order components. Therefore, we can conclude that the main structural changes in the curves can be reduced to three different components and hence at most three different length scales can be extracted from the data.

Figures E.2a - E.2b show how the scores of the first three depend on the positions

(a) 1. Component

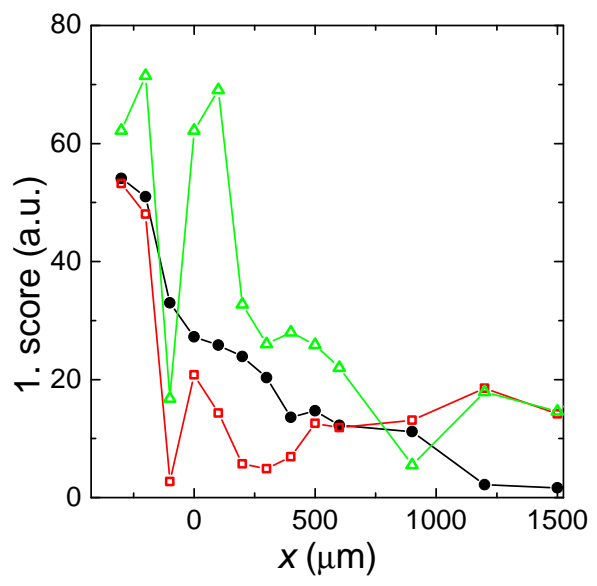

(b) 2. Component

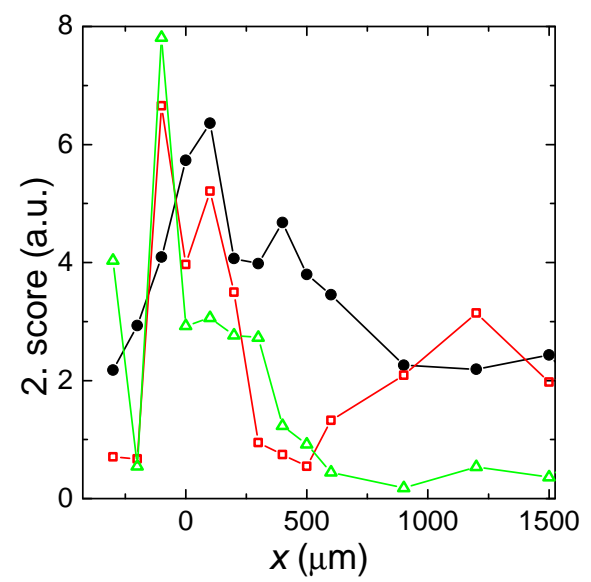

(c) 3. Component

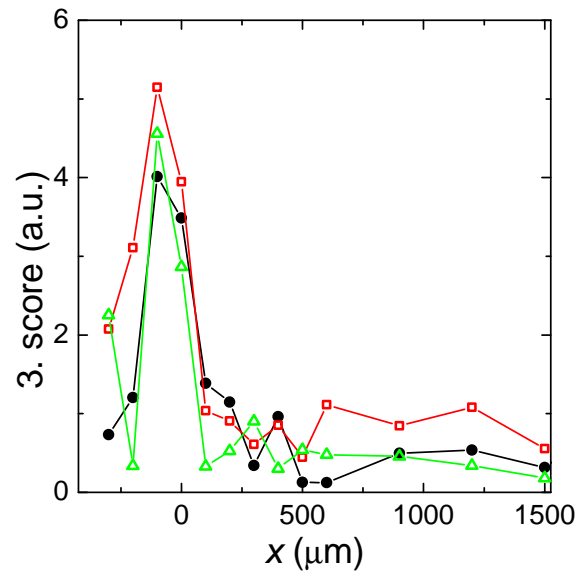

Figure E.2: (a) - (c) The absolute scores of first three components of the primary component analysis for different positions in the device for flow conditions as described in figure 7.1 (black circles), at flow rates reduced by a factor of 2 (red squares) and at flow rates reduced by a factor of 4 (green triangles). The closed symbols represent the component and the open symbol the negative part of the component.

in flow for all three flow rates considered. As the individual components for the flow conditions differ, so do their respective scores. The first score shows an initial decay but afterwards rises again (for the fastest flow speed outside of the shown region) 
and remains at non-zero values also for longer distances. For the fastest flow case we can estimate the overall decay length to about $350 \mu \mathrm{m}$, which is comparable to the decay length estimate from the mean of the first derivative (see table 7.1). For the other flow speeds due to the more complex location dependence, this is not possible. The scores of the second components all show an initial increase followed by a subsequent decrease to close to zero (outside of plotted range) with a local maximum around $x=0$. For the slowest flow rate, a length scale of the decay towards zero of $500 \mu \mathrm{m}$ can be identified which corresponds to the scale on which the second derivative changes. The third component has its maximum score at $x=-100 \mu \mathrm{m}$, i.e. at the first measurement location after the protein inlet and decays quickly after that with a decay length comparable to that of the mean of the second derivative. 


\section{List of abbreviations}

IF Intermediate filament

SAXS Small angle X-ray scattering

ULF Unit-length-filament (an intermediate of IF formation)

TEM Transmission electron microscopy

Y117L A vimentin mutant that stops assembly at the ULF stage

ATP Adenosintriphosphat

GTP Guanosintriphosphat

AFM Atomic force microscopy

A Absorbance of a sample

$l_{s}$ Thickness of a sample

$\epsilon$ Absorption coefficient of a molecule

$c$ Concentration of a molecule

$L_{D} \quad$ Sample to detector distance

$\theta$ Angle between incoming photon and scattered photon in SAXS

$\Phi$ Azimuthal angle in the detector plane

$\vec{k} \quad$ Momentum of incoming photon

$\overrightarrow{k^{\prime}} \quad$ Momentum of scattered photon

$\vec{q}$ Momentum transfer in scattering

$\lambda$ Wavelength of a photon

$\vec{r}$ Coordinate in real space

$I(\vec{q}) \quad$ Scatterd intensity in dependence of $\vec{q}$

$S(\vec{q}) \quad$ Intermolecular structure factor in dependence of $\vec{q}$

$F(\vec{q}) \quad$ Intramolecular form factor in dependence of $\vec{q}$

$\rho(\vec{r}) \quad$ Electron density distribution

$\gamma(\vec{r}) \quad$ Auto-correlation of $\rho(\vec{r})$

$\gamma(r) \quad$ Orientational mean of $\gamma(\vec{r})$

$p(r) \quad$ Pair distance distribution function

$D, D_{\max }$ maximum distance in a molecule 
PDDF Pair distance distribution function

$L$ Length of a rod or filament

$\rho_{c}(\vec{r})$ Electron density distribution of the cross-section of a rod

$F_{c}(q)$ Form factor of the cross-section of a rod

$P_{L} \quad$ Longitudinal component of the scattering of a rod

$\operatorname{Si}(x) \quad$ Sine integral

$\gamma_{c}(r)$ Orientational mean of the auto-correlation of $\rho_{c}(\vec{r})$

$p_{c}(r)$ Pair distance distribution function of the cross-section of a rod

WAXS Wide angle X-ray scattering

$R$ Radius of a cylinder

$\rho_{\text {core }}$ Electron density of the core in the core-corona model

$G_{n}$ Radius of gyration of the $n$th chain of the corona

$\rho_{\text {gaussian }}$ Electron density of a Gaussian chain

$p_{n} \quad$ Position of the $n$th chain in the corona

$N_{U L F} \quad$ Number of ULFs in a filament

$N_{G} \quad$ Number of Gaussian chains in the corona

$I_{s} \quad$ Scattering from the core in the core-corona model

$I_{c} \quad$ Scattering from individual chains in the core-corona model

$I_{s c} \quad$ Scattering between the core and the chains in the core-corona model

$I_{c c} \quad$ Scattering from inbetween chains in the core-corona model

$\Psi \quad$ Form factor amplitude of a Gaussian chain

$\beta_{s} \quad$ Forward scattering amplitude of a single ULF

$\beta_{c} \quad$ Forward scattering amplitude of a Gaussian chain in the corona

$d R$ Distance between the corona and the filament core

$l$ Length of a ULF in a filament

$\gamma$ Forward scattering amplitude of a ULF per unit length

$b$ Foward scattering of a Gaussian chain in terms of the scattering from a ULF

$\lambda$ Mean distance between chains along the filament

$F_{F}(q)$ Complete form-factor of the core-corona model

$F_{T}(q)$ Extension of $F_{F}(q)$ which includes a tetrameric component

$M_{L} \quad$ Mass per length of a filament

$\Delta \rho$ Mean electron density contrast between a molecule and its solvent

$\vec{v} \quad$ Velocity field of a flow

$p$ Pressure difference in a flow

UV Ultraviolet light 
$\eta \quad$ Viscosity of a fluid

$\vec{f}$ External forces on a fluid

$U$ Characteristic velocity of a flow

$L_{F} \quad$ Characteristic length scale of a flow

Re Reynolds number

$D$ Diffusion constant of a solute

$\vec{j}_{c o} \quad$ Convective flow of a solute

$\vec{j}_{d i} \quad$ Diffusive flow of a solute

$L_{T} \quad$ Characteristic length scale of a transport process

$t_{c o}$ Typical time-scale of convective flow

$t_{d i}$ Typical time-scale of diffusive flow

Pe Péclet number

PDMS Polydimethylsiloxan

NOA 81 Norland optical adhesive

FEM Finite element method

PDE Partial differential equation

$D_{\text {vim }}$ Diffusion constant of vimentin

$D_{K C l}$ Diffusion constant of potassium chloride

$\Theta(x) \quad$ Step-function

$\eta_{\text {tet }} \quad$ Viscosity of tetrameric vimentin solution

$\eta_{\text {vim }}$ Viscosity of filamentous vimentin solution

$c_{v i m}$ Vimentin concentration

$c_{s}$ Potassium chloride concentration

$\eta_{v}, \eta_{s}$ Parameters for viscosity model

$c_{\text {min }}, c_{0}, p$ Parameters for viscosity model

$x$ Coordinates in microfluidic device along protein flow axis

$y$ Coordinates in microfluidic device along salt buffer flow axis

$z$ Coordinates in microfluidic perpendicular to main flow directions

$\Delta x, \Delta y \quad \mathrm{X}$-ray spot size

$V$ Probe volume of X-ray beam

$I_{l} \quad$ Local SAXS signal

$t$ Assembly time of vimentin

$Q$ Typical flow rate

$w$ Channel width

$h$ Channel height 
$k_{f}$ Reaction rate

$\Delta G_{A}$ Activation energy

$T$ Temperature

$k_{b} \quad$ Boltzmann's constant

$\xi \quad$ Local friction coefficient

a Linear charge density

$\zeta$ Linear charge density parameter

$\lambda_{b}$ Bjerrum length

$Z$ Charge of an ion

$d$ Distance between vimentin tetramers in an octomer or hexadecamer 


\section{List of Figures}

2.1 The three main filamentous components of cells . . . . . . . . . . . 4

2.2 Structure of the vimentin dimer . . . . . . . . . . . . . 5

2.3 Proposed structures for the vimentin tetramer and ULF . . . . . . . 5

2.4 Assembly model for vimentin . . . . . . . . . . . . . . . . 8

3.1 Basic setup of a SAXS experiment . . . . . . . . . . . . . . 12

3.2 Schematics of the B1 setup . . . . . . . . . . . . . 16

3.3 Correction for background scattering at B1 . . . . . . . . . . . 17

3.4 Schematics of the cSAXS setup . . . . . . . . . . . . 18

3.5 Correction for background scattering at cSAXS . . . . . . . . 18

4.1 Comparison of the scattering from different vimentin preparations . . 24

4.2 Scattering of vimentin at low salt and at $50 \mathrm{mM} \mathrm{KCl}$ for different protein concentrations . . . . . . . . . . . . . . 26

4.3 Scattering of vimentin at $2.5 \mathrm{mM} \mathrm{MgCl}_{2}$ and at $80 \mathrm{mM} \mathrm{KCl}, 2.5 \mathrm{mM}$ $\mathrm{MgCl}_{2}$ for different protein concentrations . . . . . . . . . . . 26

4.4 Scattering of the vimentin mutant $Y 117 \mathrm{~L}$ at $50 \mathrm{mM} \mathrm{KCl}$ for different protein concentrations . . . . . . . . . . . . . . . 27

4.5 Changes in the scattering induced by a single cationic species . . . . . 29

4.6 Changes in the scattering induced by a $\mathrm{MgCl}_{2} \ldots \ldots$. . . . . . . 31

4.7 Changes in the scattering of $Y 117 L$ induced by $\mathrm{KCl}$. . . . . . . . . . 33

4.8 Changes in the scattering of $Y 117 \mathrm{~L}$ induced by $\mathrm{KCl}$ at $2.5 \mathrm{mM} \mathrm{MgCl} 234$

4.9 Comparison between wild type vimentin and $Y 117 \mathrm{~L} \ldots . . \ldots . . . .35$

4.10 Theoretical scattering curves of perfect cylinders . . . . . . . . . . . . 37 
4.11 Guinier fits . . . . . . . . . . . . . . . . . . 39

4.12 Results of Guinier fits . . . . . . . . . . . . . . . . . . . . 40

4.13 Guinier fits of $Y 117 L \ldots \ldots \ldots 42$

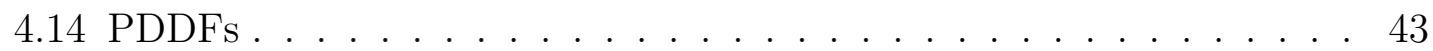

4.15 Radii of gyration from the PDDF . . . . . . . . . . . . 45

4.16 PDDFs of $Y 117 L \ldots \ldots \ldots \ldots$

4.17 Comparison of fits to data . . . . . . . . . . . . . . . . . . 48

4.18 Electron density model for vimentin IF . . . . . . . . . . . . . . . . 49

4.19 Fits to equation $4.9 \ldots \ldots \ldots \ldots$. . . . . . . . . . . . . . . 52

4.20 Fits to equation 4.9 with polydisperse cylinder cores . . . . . . . . . . 54

4.21 Contribution of tetrameric vimentin . . . . . . . . . . 56

4.22 Cylinder radii and corona radii of gyration for wild type vimentin in the presence of a single ionic species . . . . . . . . . . . . 57

4.23 Cylinder radii and corona radii of gyration for wild type vimentin in the presence of both potassium and magnesium . . . . . . . . 58

4.24 Cylinder radii and corona radii of gyration for the $Y 177 \mathrm{~L}$ mutant in the presence of both potassium and magnesium . . . . . . . . 58

4.25 Scaling factors of wild type vimentin for a single cation species . . . . 59

4.26 Scaling factors of wild type vimentin for a single cation species . . . . 60

4.27 Scaling factors of the $Y 11^{7} \mathrm{~L}$ mutant . . . . . . . . . . . . 60

4.28 Connection between $\lambda$ and $R \ldots \ldots$. . . . . . . . . 61

4.29 Cross-sectional radii of gyration of wild type vimentin based on equa-

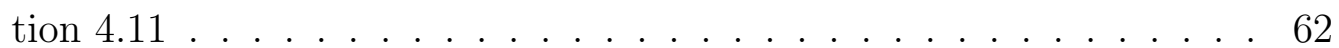

4.30 Cross-sectional radii of gyration of the $Y 117 \mathrm{~L}$ mutant based on equation $4.11 \ldots \ldots \ldots \ldots \ldots \ldots$

4.31 Form factors for different cross-sections . . . . . . . . . . . . . . 68

4.32 Forward scattering of the central rod vs. radius of the central rod . . 71

4.33 Overview of the cross-sectional radius of gyration . . . . . . . . . 72

5.1 Schematic of the microfluidic layout used for studying the vimentin assembly . . . . . . . . . . . . . . . . . . . 79 
5.2 Sketch of the microfluidic channel geometry with pre-focusing . . . . 79

5.3 Three dimensional sketch of the microfluidic channel geometry . . . . 80

5.4 Comparison between SAXS signals of Kapton film and NOA 81 . . . 82

5.5 Fabrication of microfluidic devices . . . . . . . . . . . . . . . . 84

5.6 Device production steps - wafer and PDMS stamp . . . . . . . . . . 85

5.7 Device production steps - NOA 81 films . . . . . . . . . . 86

5.8 A microfluidic device from NOA $81 \ldots$. . . . . . . . . . . . . 87

5.9 Characterization of flow with fluorescein . . . . . . . . . . . 89

6.1 Results of vimentin flow simulation . . . . . . . . . . . . . 98

6.2 Exemplary salt concentration and assembly time ensembles . . . . . . 99

6.3 Exemplary salt concentration and assembly time histograms . . . . . 100

6.4 Exemplary salt concentration and assembly time means . . . . . . . . 101

6.5 Results of vimentin flow simulation, comparison of different protein inlet heights . . . . . . . . . . . . . . . . . . . 103

6.6 Exemplary salt concentration and assembly time ensembles for different protein inlet heights . . . . . . . . . . . . . . . . . . . 104

6.7 Exemplary salt concentration and assembly time histograms for different protein inlet heights . . . . . . . . . . . . . . . . . . . 105

6.8 Exemplary salt concentration and assembly time ensembles for different absolute flow speeds . . . . . . . . . . . . . . . . . . 107

6.9 Salt concentration versus assembly time for different flow speeds . . . 108

6.10 Exemplary salt concentration and assembly time ensembles for different relative flow speeds . . . . . . . . . . . . . . 109

6.11 Exemplary salt concentration and assembly time ensembles for different buffer salt concentrations . . . . . . . . . . . . . . . . . . . 110

6.12 Exemplary salt concentration and assembly time ensembles for different probe sizes . . . . . . . . . . . . . . . . . . . 111

6.13 Results of vimentin flow simulation - effect of pre-focusing . . . . . . 113

6.14 Exemplary salt concentration and assembly time ensembles - effect of pre-focusing . . . . . . . . . . . . . . . . . . . 114

6.15 Exemplary salt concentration and assembly time histograms . . . . . 115 
6.16 Example for the extrapolation of single streamlines . . . . . . . 117

6.17 Example for the extrapolation of single streamlines . . . . . . . . 118

6.18 Estimate of SAXS curves in microflow based on stationary wild type data . . . . . . . . . . . . . . . . . . . . . . . . . . . . . . . . .

6.19 Estimate of SAXS curves in microflow based on stationary Y117L data121

6.20 Estimate of SAXS curves in microflow based on length adjusted stationary $Y 117 L$ data . . . . . . . . . . . . . . . . . . . 122

6.21 Modeled kinetics of vimentin assembly . . . . . . . . . . . . . . . . 124

6.22 Modeled scattering curves of octamers and hexadecamers . . . . . . . 125

6.23 Estimate of SAXS curves in microflow based on equations 6.5 to 6.12126

7.1 SAXS curves in microflow near the intersection . . . . . . . . . 128

7.2 Comparison of equilibrium and in flow low salt data . . . . . . . . . . 129

7.3 SAXS curves in microflow further downstream . . . . . . . . . . . . 130

7.4 Comparison of equilibrium and in flow high salt data . . . . . . . 131

7.5 SAXS curves in microflow near the intersection . . . . . . . . . . . 132

7.6 SAXS curves in microflow further downstream . . . . . . . . . . . 133

7.7 SAXS curves in microflow near the intersection . . . . . . . . . . 133

7.8 SAXS curves in microflow further downstream . . . . . . . . . . . . . 134

7.9 SAXS curves in microflow near the intersection . . . . . . . . . . 135

7.10 SAXS curves in microflow further downstream . . . . . . . . . . 135

7.11 Comparison of equilibrium and in-flow low salt data . . . . . . . . 136

7.12 SAXS curves in microflow for the pre-focusing geometry . . . . . 138

7.13 SAXS curves of the $Y 117 L$ mutant protein in microflow for the prefocusing geometry . . . . . . . . . . . . . . . . . . . 139

7.14 Comparison of the scattering curve of the wild type protein and the Y117L mutant protein in flow . . . . . . . . . . . . . . . . 139

7.15 Cross-sectional radius of gyration in flow . . . . . . . . . . . . 141

7.16 Quantification of changes in the SAXS curve - smoothing and derivatives 144

7.17 Quantification of changes in the SAXS curve - first derivative . . . . . 145

7.18 Quantification of changes in the SAXS curve - second derivative . . . 146 
7.19 From length scales to time and concentration scales for the radius of gyration . . . . . . . . . . . . . . . . . . . . . . 148

7.20 From length scales to time and concentration scales: first derivative . 150

7.21 From length scales to time and concentration scales: second derivative 151

8.1 Sketch of the energy landscape for compaction . . . . . . . . . . . 156

8.2 The total excess charge of a vimentin tetramer . . . . . . . . . . . 159

E.1 Quantification of changes in the SAXS curve . . . . . . . . . . . . . 192

E.2 Quantification of changes in the SAXS curve . . . . . . . . . . . . 193 


\section{Bibliography}

[1] B. Alberts, A. Johnson, J. Lewis, M. Raff, K. Roberts, and P. Walter. Molecular Biology of the Cell. Garland Science, 2007.

[2] L. G. Goldfarb, P. Vicart, H. H. Goebel, and M. C. Dalakas. Desmin myopathy. Brain, 127(4):723-734, 2004.

[3] C. Lavedan, S. Buchholtz, R. L. Nussbaum, R. L. Albin, and M. H. Polymeropoulos. A mutation in the human neurofilament M gene in Parkinson's disease that suggests a role for the cytoskeleton in neuronal degeneration. Neuroscience Letters, 322(1):57-61, 2002.

[4] M. Müller, S. S. Bhattacharya, T. Moore, Q. Prescott, T. Wedig, H. Herrmann, and T. M. Magin. Dominant cataract formation in association with a vimentin assembly disrupting mutation. Human Molecular Genetics, 18(6):1052-1057, 2009.

[5] A. Kölsch, R. Windoffer, T. Wurflinger, T. Aach, and R. E. Leube. The keratin-filament cycle of assembly and disassembly. Journal of Cell Science, 123(13):2266-2272, 2010.

[6] T. D. Pollard and G. G. Borisy. Cellular motility driven by assembly and disassembly of actin filaments. Cell, 112(4):453-465, 2003.

[7] T. D. Pollard, W. C. Earnshaw, and J. Lippincott-Schwartz. Cell Biology Das Orginal mit Übersetzungshilfen. Springer, Berlin, 2nd edition, 2007.

[8] K. C. Neuman and A. Nagy. Single-molecule force spectroscopy: optical tweezers, magnetic tweezers and atomic force microscopy. Nat Meth, 5(6):491-505, 2008.

[9] S. Köster, J. B. Leach, B. Struth, T. Pfohl, and J. Y. Wong. Visualization of flow-aligned type I collagen self-assembly in tunable $\mathrm{pH}$ gradients. Langmuir, 23(2):357-359, 2007. 
[10] H. M. Evans, E. Surenjav, C. Priest, S. Herminghaus, R. Seemann, and T. Pfohl. In situ formation, manipulation, and imaging of droplet-encapsulated fibrin networks. Lab on a Chip, 9(13):1933-1941, 2009.

[11] Bernd Nöding and Sarah Köster. Intermediate filaments in small configuration spaces. Physical Review Letters, 108:088101, 2012.

[12] M. G. Mendez, S. I. Kojima, and R. D. Goldman. Vimentin induces changes in cell shape, motility, and adhesion during the epithelial to mesenchymal transition. Faseb Journal, 24(6):1838-1851, 2010.

[13] Brian T. Helfand, Lynne Chang, and Robert D. Goldman. Intermediate filaments are dynamic and motile elements of cellular architecture. Journal of Cell Science, 117(2):133-141, 2004.

[14] N.A. Campbell and J. B. Reece. Biology. Pearson Eduacation, San Francisco, California, USA, 7 edition, 2005.

[15] B. Hinz, G. Celetta, J. J. Tomasek, G. Gabbiani, and C. Chaponnier. Alphasmooth muscle actin expression upregulates fibroblast contractile activity. Molecular Biology of the Cell, 12(9):2730-2741, 2001.

[16] I. Sumara, M. Quadroni, C. Frei, M. H. Olma, G. Sumara, R. Ricci, and M. Peter. A Cul3-based E3 ligase removes aurora B from mitotic chromosomes, regulating mitotic progression and completion of cytokinesis in human cells. Developmental Cell, 12(6):887-900, 2007.

[17] H. Herrmann, S. V. Strelkov, P. Burkhard, and U. Aebi. Intermediate filaments: primary determinants of cell architecture and plasticity. Journal of Clinical Investigation, 119(7):1772-1783, 2009.

[18] M. Hesse, T. M. Magin, and K. Weber. Genes for intermediate filament proteins and the draft sequence of the human genome: novel keratin genes and a surprisingly high number of pseudogenes related to keratin genes 8 and 18 . Journal of Cell Science, 114(14):2569-2575, 2001.

[19] E. Schmid, D. L. Schiller, C. Grund, J. Stadler, and W. W. Franke. Tissue type-specific expression of intermediate filament proteins in a cultured epithelial cell line from bovine mammary gland. The Journal of Cell Biology, 96(1):37-50, 1983. 
[20] L. Chang and R. D. Goldman. Intermediate filaments mediate cytoskeletal crosstalk. Nature Reviews Molecular Cell Biology, 5(8):601-613, 2004.

[21] R. V. Zackroff and R. D. Goldman. In vitro assembly of intermediate filaments from baby hamster kidney (BHK-21) cells. Proceedings of the National Academy of Sciences, 76(12):6226-6230, 1979.

[22] J. C. R. Jones, A. E. Goldman, P. M. Steinert, S. Yuspa, and R. D. Goldman. Dynamic aspects of the supramolecular organization of intermediate filament networks in cultured epidermal cells. Cell Motility, 2(3):197-213, 1982.

[23] H. Herrmann, M. Haner, M. Brettel, N. O. Ku, and U. Aebi. Characterization of distinct early assembly units of different intermediate filament proteins. Journal of Molecular Biology, 286(5):1403-1420, 1999.

[24] W. Renner, W. W. Franke, E. Schmid, N. Geisler, K. Weber, and E. Mandelkow. Reconstitution of intermediate-sized filaments from denatured monomeric vimentin. Journal of Molecular Biology, 149(2):285-306, 1981.

[25] K. R. Rogers, H. Herrmann, and W. W. Franke. Characterization of disulfide crosslink formation of human vimentin at the dimer, tetramer, and intermediate filament levels. Journal of Structural Biology, 117(1):55-69, 1996.

[26] D. A. D. Parry, S. V. Strelkov, P. Burkhard, U. Aebi, and H. Herrmann. Towards a molecular description of intermediate filament structure and assembly. Experimental Cell Research, 313(10):2204-2216, 2007.

[27] H. Herrmann, M. Haner, M. Brettel, S. A. Müller, K. N. Goldie, B. Fedtke, A. Lustig, W. W. Franke, and U. Aebi. Structure and assembly properties of the intermediate filament protein vimentin: The role of its head, rod and tail domains. Journal of Molecular Biology, 264(5):933-953, 1996.

[28] S. V. Strelkov, H. Herrmann, N. Geisler, T. Wedig, R. Zimbelmann, U. Aebi, and P. Burkhard. Conserved segments $1 \mathrm{~A}$ and $2 \mathrm{~B}$ of the intermediate filament dimer: their atomic structures and role in filament assembly. Embo Journal, 21(6):1255-1266, 2002.

[29] S. Nicolet, H. Herrmann, U. Aebi, and S. V. Strelkov. Atomic structure of vimentin coil 2. Journal of Structural Biology, 170(2):369-376, 2010.

[30] A. V. Sokolova, L. Kreplak, T. Wedig, N. Mücke, D. I. Svergun, H. Herrmann, U. Aebi, and S. V. Strelkov. Monitoring intermediate filament assembly by 
small-angle X-ray scattering reveals the molecular architecture of assembly intermediates. Proceedings of the National Academy of Sciences of the United States of America, 103(44):16206-16211, 2006.

[31] L. Kreplak, K. Richter, U. Aebi, and H. Herrmann. Electron microscopy of intermediate filaments: Teaming up with atomic force and confocal laser scanning microscopy. In Introduction to Electron Microscopy for Biologists, volume 88 of Methods in Cell Biology, pages 273-297. Elsevier Academic Press Inc, San Diego, 2008.

[32] N. Mücke, T. Wedig, A. Burer, L. N. Marekov, P. M. Steinert, J. Langowski, U. Aebi, and H. Herrmann. Molecular and biophysical characterization of assembly-starter units of human vimentin. Journal of Molecular Biology, 340(1):97-114, 2004.

[33] N. Hirokawa, M. A. Glicksman, and M. B. Willard. Organization of mammalian neurofilament polypeptides within the neuronal cytoskeleton. The Journal of Cell Biology, 98(4):1523-1536, 1984.

[34] H. Herrmann and U. Aebi. Intermediate filament assembly: fibrillogenesis is driven by decisive dimer-dimer interactions. Current Opinion in Structural Biology, 8(2):177-185, 1998.

[35] R. Kirmse, S. Portet, N. Mücke, U. Aebi, H. Herrmann, and J. Langowski. A quantitative kinetic model for the in vitro assembly of intermediate filaments from tetrameric vimentin. Journal of Biological Chemistry, 282(25):1856318572, 2007.

[36] I. Hofmann, H. Herrmann, and W. W. Franke. Assembly and structure of calcium-induced thick vimentin filaments. European Journal of Cell Biology, 56(2):328-341, 1991.

[37] M. Kooijman, M. Bloemendal, P. Traub, R. van Grondelle, and H. van Amerongen. Transient electric birefringence study of intermediate filament formation from vimentin and glial fibrillary acidic protein. Journal of Biological Chemistry, 272(36):22548-22555, 1997.

[38] Y. C. Lin, C. P. Broedersz, A. C. Rowat, T. Wedig, H. Herrmann, F. C. MacKintosh, and D. A. Weitz. Divalent cations crosslink vimentin intermediate filament tail domains to regulate network mechanics. Journal of Molecular Biology, 399(4):637-644, 2010. 
[39] Y. C. Lin, N. Y. Yao, C. P. Broedersz, H. Herrmann, F. C. MacKintosh, and D. A. Weitz. Origins of elasticity in intermediate filament networks. Physical Review Letters, 104(5):058101, 2010.

[40] S. Köster, Y. C. Lin, H. Herrmann, and D. A. Weitz. Nanomechanics of vimentin intermediate filament networks. Soft Matter, 6(9):1910-1914, 2010.

[41] C. Dammann, B. Nöding, and S. Köster. Vimentin networks at tuneable ionconcentration in microfluidic drops. Biomicrofluidics, 6:022009, 2012.

[42] M. H. Stromer, M. A. Ritter, Y. Y. S. Pang, and R. M. Robson. Effect of cations and temperature on kinetics of desmin assembly. Biochemical Journal, 246(1):75-81, 1987.

[43] N. Mücke. Biophysikalische Untersuchungen des muskelspezifischen Intermediärfilament-Proteins Desmins und Mutanten. PhD thesis, Universität Mannheim, 2008.

[44] S. Winheim, A. R. Hieb, M. Silbermann, E.-M. Surmann, T. Wedig, H. Herrmann, J. Langowski, and N. Mücke. Deconstructing the late phase of vimentin assembly by total internal reflection fluorescence microscopy (TIRFM). PLoS ONE, 6(4):e19202, 2011.

[45] M. Meier, G. P. Padilla, H. Herrmann, T. Wedig, M. Hergt, T. R. Patel, J. Stetefeld, U. Aebi, and P. Burkhard. Vimentin coil 1A-a molecular switch involved in the initiation of filament elongation. Journal of Molecular Biology, $390(2): 245-261,2009$.

[46] S. Georgakopoulou, D. Moller, N. Sachs, H. Herrmann, and U. Aebi. Near-UV circular dichroism reveals structural transitions of vimentin subunits during intermediate filament assembly. Journal of Molecular Biology, 386(2):544-553, 2009

[47] R. Kirmse. Studium der Wachstumskinetik von Intermediärfilamenten mit Hilfe von Vimentin. PhD thesis, Universität Heidelberg, 2007.

[48] S. Portet, N. Mücke, R. Kirmse, J. Langowski, M. Beil, and H. Herrmann. Vimentin intermediate filament formation: In Vitro measurement and mathematical modeling of the filament length distribution during assembly. Langmuir, 25(15):8817-8823, 2009. 
[49] P. M. Steinert, W. W. Idler, and S. B. Zimmerman. Self-assembly of bovine epidermal keratin filaments IN VITRO. Journal of Molecular Biology, 108(3):547-567, 1976.

[50] H. Fraenkel-Conrat and Robley C. Williams. Reconstitution of active tobacco mosaic virus from its inactive protein and nucleic acid components. Proceedings of the National Academy of Sciences, 41(10):690-698, 1955.

[51] H. Frielinghaus. Amphiphilc systems. In J. K.G. Dhont, G. Gompper, P. R. Lang, D. Richter, M.l Ripoll, D. Willbold, and R. Zorn, editors, Macromolecular Systems in Soft and Living Matter, volume 20 of Reihen Schlüsseltechnologien. Schriften des Forschungzentrums Jülich, Jülich, 2011.

[52] B. Strodel. Protein assembly. In J. K.G. Dhont, G. Gompper, P. R. Lang, D. Richter, M. Ripoll, D. Willbold, and R. Zorn, editors, Macromolecular Systems in Soft and Living Matter, volume 20 of Reihen Schlüsseltechnologien. Schriften des Forschungzentrums Jülich, Jülich, 2011.

[53] J. Gsponer and M. Vendruscolo. Theoretical approaches to protein aggregation. Protein and Peptide Letters, 13(3):287-293, 2006.

[54] P.R. Lange. Chraged macromolecules. In J. K.G. Dhont, G. Gompper, P. R. Lang, D. Richter, M. Ripoll, D. Willbold, and R. Zorn, editors, Macromolecular Systems in Soft and Living Matter, Reihen Schlüsseltechnologien. Schriften des Forschungzentrums Jülich, Jülich, 2011.

[55] D. A. Jacques and J. Trewhella. Small-angle scattering for structural biology Expanding the frontier while avoiding the pitfalls. Protein Science, 19(4):642$657,2010$.

[56] H. Herrmann, I. Hofmann, and W. W. Franke. Identification of a nonapeptide motif in the vimentin head domain involved in intermediate filament assembly. Journal of Molecular Biology, 223(3):637-650, 1992.

[57] A. Aitken and M. Learmonth. Protein determination by UV absorption. In J.M. Walker, editor, Protein protocols handbook. Humana Press Inc., Totowa, NJ, 1996.

[58] S. Doniach. Changes in biomolecular conformation seen by small angle X-ray scattering. Chemical Reviews, 101(6):1763-1778, 2001. 
[59] D. I. Svergun and M. H. J. Koch. Small-angle scattering studies of biological macromolecules in solution. Reports on Progress in Physics, 66(10):1735-1782, 2003.

[60] L. Pollack, M. W. Tate, A. C. Finnefrock, C. Kalidas, S. Trotter, N. C. Darnton, L. Lurio, R. H. Austin, C. A. Batt, S. M. Gruner, and S. G. J. Mochrie. Time resolved collapse of a folding protein observed with small angle X-ray scattering. Physical Review Letters, 86(21):4962-4965, 2001.

[61] D. J. Segel, A. Bachmann, J. Hofrichter, K. O. Hodgson, S. Doniach, and T. Kiefhaber. Characterization of transient intermediates in lysozyme folding with time-resolved small-angle X-ray scattering. Journal of Molecular Biology, 288(3):489-499, 1999.

[62] Y. Lu, C. M. Jeffries, and J. Trewhella. Invited review: Probing the structures of muscle regulatory proteins using small-angle solution scattering. Biopolymers, 95(8):505-516, 2006.

[63] K. Schneider, N. E. Zafeiropoulos, and M. Stamm. In situ investigation of structural changes during deformation and fracture of polymers by synchrotron SAXS and WAXS. Advanced Engineering Materials, 11(6):502-506, 2009.

[64] S. Castorph, D. Riedel, L. Arleth, M. Sztucki, R. Jahn, M. Holt, and T. Salditt. Structure parameters of synaptic vesicles quantified by small-angle X-ray scattering. Biophysical Journal, 98(7):1200-1208, 2010.

[65] M. Heinen, F. Zanini, F. Roosen-Runge, D. Fedunova, F. J. Zhang, M. Hennig, T. Seydel, R. Schweins, M. Sztucki, M. Antalik, F. Schreiber, and G. Nägele. Viscosity and diffusion: crowding and salt effects in protein solutions. Soft Matter, 8(5):1404-1419, 2012.

[66] Gene E. Ice, John D. Budai, and Judy W. L. Pang. The race to X-ray microbeam and nanobeam science. Science, 334(6060):1234-1239, 2011.

[67] P. Lindner and Th. Zemb. Neutrons, X-Rays and Light. Elsevier, Amsterdam, 2002.

[68] E. Kaler. Small-angle scattering from colloidal dispersions. Journal of Applied Crystallography, 21(6):729-736, 1988.

[69] O. Glatter and O. Kratky. Small Angle X-ray Scattering. Academic Press, London, 1982. 
[70] C. G. De Kruif, W. J. Briels, R. P. May, and A. Vrij. Hard-sphere colloidal silica dispersions. the structure factor determined with SANS. Langmuir, 4(3):668-676, 1988.

[71] R. Dootz, H. Evans, S. Köster, and T. Pfohl. Rapid prototyping of X-ray microdiffraction compatible continuous microflow foils. Small, 3(1):96-100, 2007.

[72] Th. Neugebauer. Berechnung der Lichtzerstreuung von Fadenkettenlösungen. Annalen der Physik, 434(7-8):509-533, 1943. 1521-3889.

[73] J. S. Pedersen and P. Schurtenberger. Scattering functions of semiflexible polymers with and without excluded volume effects. Macromolecules, 29(23):7602$7612,1996$.

[74] P. Mittelbach and G. Porod. Zur Röntgenkleinwinkelstreuung verdünnter kolloidaler Systeme VI. die Berechnung von Streukurven von elliptischen Zylindern und Hohlzylindern. Acta Physica Austriaca, 14(3):405, 1961.

[75] H. G. Haubold, K. Gruenhagen, M. Wagener, H. Jungbluth, H. Heer, A. Pfeil, H. Rongen, G. Brandenberg, R. Moeller, J. Matzerath, P. Hiller, and H. Halling. JUSIFA - a new user-dedicated ASAXS beamline for materials science. Review of Scientific Instruments, 60(7):1943-1946, 1989. Part $2 \mathrm{~A}$.

[76] U. Vainio. Conducting experiments a B1. "http://hasylab.desy.de/ facilities/doris_iii/beamlines/b1/index_eng.html", 2011.

[77] Lee Makowski. Characterization of proteins with wide-angle X-ray solution scattering (WAXS). Journal of Structural and Functional Genomics, 11(1):9$19,2010$.

[78] http://www.psi.ch/sls/csaxs/csaxs.

[79] J. S. Pedersen. Analysis of small-angle scattering data from colloids and polymer solutions: modeling and least-squares fitting. Advances in Colloid and Interface Science, 70:171-210, 1997.

[80] O. Glatter. Evaluation of small-angle scattering data from lamellar and cylindrical particles by the indirect transformation method. Journal of Applied Crystallography, 13(DEC):577-584, 1980. 
[81] D. I. Svergun. Determination of the regularization parameter in indirecttransform methods using perceptual criteria. Journal of Applied Crystallography, 25:495-503, 1992.

[82] http://www.embl-hamburg.de/biosaxs/software.html.

[83] M. G. Ortore, F. Spinozzi, S. Vilasi, I. Sirangelo, G. Irace, A. Shukla, T. Narayanan, R. Sinibaldi, and P. Mariani. Time-resolved small-angle X-ray scattering study of the early stage of amyloid formation of an apomyoglobin mutant. Physical Review E, 84(6), 2011.

[84] J. S. Pedersen. Form factors of block copolymer micelles with spherical, ellipsoidal and cylindrical cores. Journal of Applied Crystallography, 33(1):637-640, 2000 .

[85] J. S. Pedersen and M. C. Gerstenberg. Scattering form factor of block copolymer micelles. Macromolecules, 29(4):1363-1365, 1996.

[86] P. Debye. Molecular-weight determination by light scattering. Journal of Physical and Colloid Chemistry, 51(1):18-32, 1947.

[87] B. Hammouda. Structure factor for starburst dendrimers. Journal of Polymer Science Part B: Polymer Physics, 30(12):1387-1390, 1992. 1099-0488.

[88] T. Yoshizaki and H. Yamakawa. Scattering functions of wormlike and helical wormlike chains. Macromolecules, 13(6):1518-1525, 1980.

[89] S. Nicolet. Coil 2 of intermediate filaments: its complete structure and inpact of desminopathy-related mutations. PhD thesis, Universität Basel, 2011.

[90] F. Zhang, M. W. A. Skoda, R. M. J. Jacobs, S. Zorn, R. A. Martin, C. M. Martin, G. F. Clark, S. Weggler, A. Hildebrandt, O. Kohlbacher, and F. Schreiber. Reentrant condensation of proteins in solution induced by multivalent counterions. Physical Review Letters, 101(14):4, 2008.

[91] F. J. Zhang, M. W. A. Skoda, R. M. J. Jacobs, R. A. Martin, C. M. Martin, and F. Schreiber. Protein interactions studied by SAXS: Effect of ionic strength and protein concentration for BSA in aqueous solutions. Journal of Physical Chemistry B, 111(1):251-259, 2007.

[92] H. Isambert and A. C. Maggs. Dynamics and rheology of actin solutions. Macromolecules, 29(3):1036-1040, 1996. 
[93] B. Hinner, M. Tempel, E. Sackmann, K. Kroy, and E. Frey. Entanglement, elasticity, and viscous relaxation of actin solutions. Physical Review Letters, 81(12):2614-2617, 1998.

[94] F. C. Mackintosh, J. Käs, and P. A. Janmey. Elasticity of semiflexible biopolymer networks. Physical Review Letters, 75(24):4425-4428, 1995.

[95] T. Lichtenstern, N. Muecke, U. Aebi, M. Mauermann, and H. Herrmann. Complex formation and kinetics of filament assembly exhibited by the simple epithelial keratins K8 and K18. Journal of Structural Biology, 177(1):54-62, 2012.

[96] H. X. Zhou. Loops in proteins can be modeled as worm-like chains. Journal of Physical Chemistry B, 105(29):6763-6766, 2001.

[97] H. Herrmann, L. Kreplak, and U. Aebi. Isolation, characterization, and in vitro assembly of intermediate filaments. In Intermediate Filament Cytoskeleton, volume 78 of Methods in Cell Biology, pages 3-24. Elsevier Academic Press Inc, San Diego, 2004.

[98] J. E. Hearst. Intrinsic viscosity of stiff-chain macromolecules. Journal of Chemical Physics, 40(6):1506, 1964.

[99] T. R. Patel, G. A. Morris, J. G. de la Torre, A. Ortega, P. Mischnick, and S. E. Harding. Molecular flexibility of methylcelluloses of differing degree of substitution by combined sedimentation and viscosity analysis. Macromolecular Bioscience, 8(12):1108-1115, 2008.

[100] N. Fakhri, D. A. Tsyboulski, L. Cognet, R. B. Weisman, and M. Pasquali. Diameter-dependent bending dynamics of single-walled carbon nanotubes in liquids. Proceedings of the National Academy of Sciences, 106(34):1421914223, 2009.

[101] C. Sachse, N. Grigorieff, and M. Fändrich. Nanoscale flexibility parameters of alzheimer amyloid fibrils determined by electron cryo-microscopy. Angewandte Chemie International Edition, 49(7):1321-1323, 2010.

[102] L.A. Feigin and D.I. Svergun. Structure analysis by small-angle X-ray and neutron scattering. Plenum Press, New York, 1987.

[103] G. Barone, Z. Sayers, D. Svergun, and M. H. J. Koch. A synchrotron radiation X-ray scattering study of aqueous solutions of native DNA. Journal of Synchrotron Radiation, 6(5):1031-1034, 1999. 
[104] Gerald S. Manning. Counterion binding in polyelectrolyte theory. Accounts of Chemical Research, 12(12):443-449, 1979.

[105] T. T. Nguyen, A. Y. Grosberg, and B. I. Shklovskii. Screening of a charged particle by multivalent counterions in salty water: Strong charge inversion. Journal of Chemical Physics, 113(3):1110-1125, 2000.

[106] M. E. Brennich, J. F. Nolting, C. Dammann, B. Nöding, S. Bauch, H. Herrmann, T. Pfohl, and S. Köster. Dynamics of intermediate filament assembly followed in micro-flow by small angle x-ray scattering. Lab on a Chip, 11(4):708-716, 2011.

[107] C. Hansen and S. R. Quake. Microfluidics in structural biology: smaller, faster \& better. Current Opinion in Structural Biology, 13(5):538-544, 2003.

[108] M. E. Kinahan, E. Filippidi, S. Köster, X. Hu, H. M. Evans, T. Pfohl, D. L. Kaplan, and J. Wong. Tunable silk: Using microfluidics to fabricate silk fibers with controllable properties. Biomacromolecules, 12(5):1504-1511, 2011.

[109] Sarah Köster and Thomas Pfohl. An in vitro model system for cytoskeletal confinement. Cell Motility and the Cytoskeleton, 66(10):771-776, 2009. 10970169.

[110] J. Clausell-Tormos, D. Lieber, J.-C. Baret, A. El-Harrak, O. J. Miller, L. Frenz, J. Blouwolff, K. J. Humphry, S. Köster, H. Duan, C. Holtze, D. A. Weitz, A. D. Griffiths, and C. A. Merten. Droplet-based microfluidic platforms for the encapsulation and screening of mammalian cells and multicellular organisms. Chemistry \& Biology, 15(5):427-437, 2008.

[111] K. Avila, D. Moxey, A. de Lozar, M. Avila, D. Barkley, and B. Hof. The onset of turbulence in pipe flow. Science, 333(6039):192-196, 2011.

[112] W. Saadi, S. Rhee, F. Lin, B. Vahidi, B. Chung, and N. Jeon. Generation of stable concentration gradients in 2D and 3D environments using a microfluidic ladder chamber. Biomedical Microdevices, 9(5):627-635, 2007.

[113] T. M. Floyd, M. A. Schmidt, and K. F. Jensen. Silicon micromixers with infrared detection for studies of liquid-phase reactions. Industrial 86 Engineering Chemistry Research, 44(8):2351-2358, 2004.

[114] D. S. Kim, S. H. Lee, T. H. Kwon, and C. H. Ahn. A serpentine laminating micromixer combining splitting/recombination and advection. Lab on a Chip, $5(7), 2005$. 
[115] J. B. Knight, A. Vishwanath, J. P. Brody, and R. H. Austin. Hydrodynamic focusing on a silicon chip: Mixing nanoliters in microseconds. Physical Review Letters, 80(17):3863-3866, 1998.

[116] S. Köster, H. M. Evans, J. Y. Wong, and T. Pfohl. An in situ study of collagen self-assembly processes. Biomacromolecules, 9(1):199-207, 2008.

[117] S. Rammensee, U. Slotta, T. Scheibel, and A. R. Bausch. Assembly mechanism of recombinant spider silk proteins. Proceedings of the National Academy of Sciences of the United States of America, 105(18):6590-6595, 2008.

[118] R. Dootz, A. Otten, S. Köster, B. Struth, and T. Pfohl. Evolution of DNA compaction in microchannels. Journal of Physics-Condensed Matter, 18(18):S639S652, 2006.

[119] R. Russell, I. S. Millettt, M. W. Tate, L. W. Kwok, B. Nakatani, S. M. Gruner, S. G. J. Mochrie, V. Pande, S. Doniach, D. Herschlag, and L. Pollack. Rapid compaction during RNA folding. Proceedings of the National Academy of Sciences of the United States of America, 99(7):4266-4271, 2002.

[120] H. Y. Park, X. Y. Qiu, E. Rhoades, J. Korlach, L. W. Kwok, W. R. Zipfel, W. W. Webb, and L. Pollack. Achieving uniform mixing in a microfluidic device: Hydrodynamic focusing prior to mixing. Analytical Chemistry, 78(13):4465-4473, 2006.

[121] J. N. Lee, C. Park, and G. M. Whitesides. Solvent compatibility of poly(dimethylsiloxane)-based microfluidic devices. Analytical Chemistry, 75(23):6544-6554, 2003.

[122] W. Hellmich, J. Regtmeier, T. T. Duong, R. Ros, D. Anselmetti, and A. Ros. Poly(oxyethylene) based surface coatings for poly(dimethylsiloxane) microchannels. Langmuir, 21(16):7551-7557, 2005.

[123] H. Makamba, J. H. Kim, K. Lim, N. Park, and J. H. Hahn. Surface modification of poly(dimethylsiloxane) microchannels. Electrophoresis, 24(21):36073619, 2003.

[124] C. Semmler and A. Alexeev. Designing structured surfaces that repel fluidborne particles. Physical Review E, 84(6):066303, 2011.

[125] R. Barret, M. Faucon, J. Lopez, G. Cristobal, F. Destremaut, A. Dodge, P. Guillot, P. Laval, C. Masselon, and J.B. Salmon. X-ray microfocussing 
combined with microfluidics for on-chip X-ray scattering measurements. Lab on a Chip, 6:494-499, 2006.

[126] L. Pollack, M. W. Tate, N. C. Darnton, J. B. Knight, S. M. Gruner, W. A. Eaton, and R. H. Austin. Compactness of the denatured state of a fast-folding protein measured by submillisecond small-angle X-ray scattering. Proceedings of the National Academy of Sciences of the United States of America, 96(18):10115-10117, 1999.

[127] T. Pfohl, A. Otten, S. Köster, R. Dootz, B. Struth, and H. M. Evans. Highly packed and oriented DNA mesophases identified using in situ microfluidic Xray microdiffraction. Biomacromolecules, 8(7):2167-2172, 2007.

[128] H. M. Evans, R. Dootz, S. Köster, B. Struth, and T. Pfohl. X-ray microdiffraction on flow-controlled biomolecular assemblies. Bulletin of the Polish Academy of Sciences-Technical Sciences, 55(2):217-227, 2007.

[129] R. Barrett, M. Faucon, J. Lopez, G. Cristobal, F. Destremaut, A. Dodge, P. Guillot, P. Laval, C. Masselon, and J.-B. Salmon. X-ray microfocussing combined with microfluidics for on-chip X-ray scattering measurements. Lab on a Chip, 6(4), 2006.

[130] A. Martel, M. Burghammer, R. J. Davies, E. Di Cola, C. Vendrely, and C. Riekel. Silk fiber assembly studied by synchrotron radiation SAXS/WAXS and raman spectroscopy. Journal of the American Chemical Society, 130(50):17070-17074, 2008.

[131] A. Martel, M. Burghammer, R. Davies, E. DiCola, P. Panine, J. B. Salmon, and C. Riekel. A microfluidic cell for studying the formation of regenerated silk by synchrotron radiation small- and wide-angle X-ray scattering. Biomicrofluidics, 2(2):7, 2008.

[132] Z. T. Cygan, J. T. Cabral, K. L. Beers, and E. J. Amis. Microfluidic platform for the generation of organic-phase microreactors. Langmuir, 21(8):3629-3634, 2005.

[133] C. Harrison, J. T. Cabral, C. M. Stafford, A. Karim, and E. J. Amis. A rapid prototyping technique for the fabrication of solvent-resistant structures. Journal of Micromechanics and Microengineering, 14(1):153-158, 2004. 
[134] S. Natarajan, D.A. Chang-Yen, and B.K. Gale. Large-area, high-aspect-ratio SU-8 molds for the fabrication of PDMS microfluidic devices. Journal of Micromechanics and Microengineering, 18(4):045021, 2008.

[135] J. C. McDonald and G. M. Whitesides. Poly(dimethylsiloxane) as a material for fabricating microfluidic devices. Accounts of Chemical Research, 35(7):491499, 2002.

[136] C. Decker and A. D. Jenkins. Kinetic approach of oxygen inhibition in ultraviolet- and laser-induced polymerizations. Macromolecules, 18(6):1241$1244,1985$.

[137] S. A. Stern, V. M. Shah, and B. J. Hardy. Structure-permeability relationships in silicone polymers. J. Polym. Sci., Part B: Polym. Phys., 25(6):1263-1298, 1987.

[138] E. M. Johnson, S. J. Clarson, H. Jiang, W. Su, J. T. Grant, and T. J. Bunning. Plasma polymerized hexamethyldisiloxane (HMDS) barrier layers. Polymer, 42(16), 2001.

[139] C. T. Culbertson, S. C. Jacobson, and J. M. Ramsey. Diffusion coefficient measurements in microfluidic devices. Talanta, 56(2):365-373, 2002.

[140] A.J. Baker. Finite element computational fluid mechanics. Hemisphere Publishing Corporation, Washington, 1983.

[141] M. E. Young, P. A. Carroad, and R. L. Bell. Estimation of diffusion-coefficients of proteins. Biotechnology and Bioengineering, 22(5):947-955, 1980.

[142] J. D'Ans, J. Bartels, P. Ten Bruggencate, A. Eucken, G. Joss, and W.A. Roth. Eigenschaften der Materie in ihren Aggregatszuständen, Transportphänomene, Kinetik, homogene Gasgleichgewichte, volume II.5 of LandoltBörnstein Zahlenwerte und Funktionen aus Physik, Chemie, Astronomie, Geophysik und Technik. Springer Verlag, 6 edition, 1969.

[143] W. J. Liu, G. E. Ice, J. Z. Tischler, A. Khounsary, C. Liu, L. Assoufid, and A. T. Macrander. Short focal length Kirkpatrick-Baez mirrors for a hard X-ray nanoprobe. Review of Scientific Instruments, 76(11):6, 2005.

[144] A.M.C. Davies and T. Fearn. Back to basics: the principles of principal component analysis. Spectroscopy Europe, 2005(2):20-23, 2005. 
[145] M. B. McCormick, P. Kouklis, A. Syder, and E. Fuchs. The roles of the rod end and the tail in vimentin IF assembly and IF network formation. The Journal of Cell Biology, 122(2), 1993.

[146] C. Fernyhough, A. J. Ryan, and G. Battaglia. pH controlled assembly of a polybutadiene-poly(methacrylic acid) copolymer in water: packing considerations and kinetic limitations. Soft Matter, 5(8):1674-1682, 2009.

[147] P. Laval, A. Crombez, and J. B. Salmon. Microfluidic droplet method for nucleation kinetics measurements. Langmuir, 25(3):1836-1841, 2009.

[148] J. D. Bryngelson, J. N. Onuchic, N. D. Socci, and P. G. Wolynes. Funnels, pathways, and the energy landscape of protein-folding - a synthesis. ProteinsStructure Function and Genetics, 21(3):167-195, 1995.

[149] H. A. Kramers. Brownian motion in a field of force and the diffusion model of chemical reactions. Physica, 7:284-304, 1940.

[150] S. A. Pabit, H. Roder, and S. J. Hagen. Internal friction controls the speed of protein folding from a compact configuration. Biochemistry, 43(39):1253212538, 2004.

[151] D. J. Needleman, M. A. Ojeda-Lopez, U. Raviv, H. P. Miller, L. Wilson, and C. R. Safinya. Higher-order assembly of microtubules by counterions: From hexagonal bundles to living necklaces. Proceedings of the National Academy of Sciences of the United States of America, 101(46):16099-16103, 2004.

[152] S. N. Watanabe and K. Yoshikawa. Bundling transition of f-actin. In MicroNanoMechatronics and Human Science, 2006 International Symposium on, pages 1-4, 2006.

[153] J. X. Tang, T. Ito, T. Tao, P. Traub, and P. A. Janmey. Opposite effects of electrostatics and steric exclusion on bundle formation by F-actin and other filamentous polyelectrolytes . Biochemistry, 36(41):12600-12607, 1997.

[154] T. Angelini, H. Liang, W. Wriggers, and G. Wong. Direct observation of counterion organization in f-actin polyelectrolyte bundles. The European Physical Journal E: Soft Matter and Biological Physics, 16(4):389-400, 2005.

[155] Z. Qin, L. Kreplak, and M. J. Buehler. Hierarchical structure controls nanomechanical properties of vimentin intermediate filaments. PLoS ONE, 4(10):e7294, 2009. 
[156] J. X. Tang and P. A. Janmey. The polyelectrolyte nature of f-actin and the mechanism of actin bundle formation. Journal of Biological Chemistry, 271(15):8556-8563, 1996.

[157] I. Minoura and E. Muto. Dielectric measurement of individual microtubules using the electroorientation method. Biophysical Journal, 90(10):3739-3748, 2006.

[158] D. Stigter. Evaluation of the counterion condensation theory of polyelectrolytes. Biophysical Journal, 69(2):380-388, 1995.

[159] J. X. Tang, S. Wong, P. T. Tran, and P. A. Janmey. Counterion induced bundle formation of rodlike polyelectrolytes. Berichte der Bunsengesellschaft für physikalische Chemie, 100(6):796-806, 1996.

[160] I.N. Bronstein and K.A. Semendjajew. Taschenbuch der Mathematik. Verlag Harri Deutsch, Zurich, Switzerland, 1966. 


\title{
Acknowledgments
}

\author{
Special thanks go to
}

Prof. Dr. Sarah Köster for the opportunity to work on this subject and the excellent support provided during this thesis

Prof. Dr. Eberhard Bodenschatz for co-refereeing this thesis and for accompanying this project as a member of the thesis committee

Prof. Dr. Tim Salditt for his continued interest in my work and for being member of the thesis committee

Prof. Dr. Helmut Grubmüller, Prof. Dr. Andreas Janshoff and Dr. Simone Techert for agreeing to be examination board members

Prof. Dr. Harald Herrmann, Dr. Norbert Mücke and Tatjana Wedig for providing the plasmids for the different vimentin types and sharing their expertise on vimentin

Dr. Anna Sokolova and Prof. Dr. Sergei Strelkov for fruitful discussion on the small angle scattering of vimentin

Christian Dammann, Jens-Friedrich Nolting and Bernd Nöding for their help in building the microfluidic devices and together with

Britta Weinhausen, Rabea Sandmann, Sarah Schwarz G. Henriques and Jannick Langfahl-Klabes for support during synchrotron beamtimes

Susanne Bauch for her assistance with everything (bio-) chemical

Dr. Ulla Vainio, Dr. Oliver Bunk, Dr. Teemu Ikonen, Dr. Manfred Burghammer and Dr. Manuel Fernández Martínez for excellent support and helpful discussions during beamtimes 
Dr. Jörg Malindretos and Jochen Herbst for their support in the clean room

Carsten Wulf and Peter Nieschalk for their support in the machine shop All members of the institute for X-ray physics for the outstanding and supportive working atmosphere

Prof. Dr. Thomas Pfohl, Dr. Simon Castorph, Dr. Sravanti Uppaluri and Dr. Eric Stellamanns for fruitful discussions on microfluidics and small angle scattering

Constantin Spille and Christian Westendorff for help with the confocal microscopy

Dr. Wiebke Möbius for electron micrographs of vimentin for quality control

The German Research Foundation for funding in the framework of SFB 755 "Nanoscale Photonic Imaging" and the Excellence Initiative 


\section{Vita}

Martha Elisabeth Brennich

\section{Personal details}

Date of birth $27^{\text {th }}$ August 1983

Place of birth Munich, Germany

Nationality German

\section{Education}

1989-1990 Grundschule an der Ichostraße, Munich, Germany

1990-1993 Grundschule an der Feldbergstraße, Munich, Germany

1993-2002 Michaeli-Gymnasium München, Munich, Germany

$2002 \quad$ Abitur

2002-2008 Studies of physics at Ludwigs-Maximilians Universität

Munich, Germany

2005-2006 Studies of physics at University of Nottingham, United Kingdom

2007-2008 Diploma thesis at the Chair for BioMolecular Optics,

Ludwigs-Maximilians Universität Munich, Germany

2009-2012 Postgraduate studies, Georg-August-Universität Götttingen,

Germany

\section{Scholarships}

2002-2007 Bayerische Begabtenförderung

2007-2008 Max-Weber-Programm of the Studienstiftung des deutschen Volkes 